\title{
Adaptive Laser Range Scanning using Quality Metrics
}

by

David K. MacKinnon

A thesis submitted to

The Faculty of Graduate Studies and Research

in partial fulfilment

of the requirements for the degree of

Doctor of Philosophy in Electrical Engineering

Department of Systems and Computer Engineering

Carleton University

Ottawa, Ontario

March 24, 2008

(C)Copyright

2008, David K. MacKinnon 


$\begin{array}{ll}\begin{array}{l}\text { Library and } \\ \text { Archives Canada }\end{array} & \begin{array}{l}\text { Bibliothèque et } \\ \text { Archives Canada }\end{array} \\ \begin{array}{l}\text { Published Heritage } \\ \text { Branch }\end{array} & \begin{array}{l}\text { Direction du } \\ \text { Patrimoine de l'édition }\end{array} \\ \begin{array}{l}\text { 395 Wellington Street } \\ \text { Ottawa ON K1A 0N4 } \\ \text { Canada }\end{array} & \begin{array}{l}\text { 395, rue Wellington } \\ \text { Ottawa ON K1A 0N4 } \\ \text { Canada }\end{array}\end{array}$

Your file Votre référence ISBN: 978-0-494-40528-4 Our file Notre référence ISBN: 978-0-494-40528-4

NOTICE:

The author has granted a nonexclusive license allowing Library and Archives Canada to reproduce, publish, archive, preserve, conserve, communicate to the public by telecommunication or on the Internet, loan, distribute and sell theses worldwide, for commercial or noncommercial purposes, in microform, paper, electronic and/or any other formats.

The author retains copyright ownership and moral rights in this thesis. Neither the thesis nor substantial extracts from it may be printed or otherwise reproduced without the author's permission.
AVIS:

L'auteur a accordé une licence non exclusive permettant à la Bibliothèque et Archives Canada de reproduire, publier, archiver, sauvegarder, conserver, transmettre au public par télécommunication ou par l'Internet, prêter, distribuer et vendre des thèses partout dans le monde, à des fins commerciales ou autres, sur support microforme, papier, électronique et/ou autres formats.

L'auteur conserve la propriété du droit d'auteur et des droits moraux qui protège cette thèse. $\mathrm{Ni}$ la thèse ni des extraits substantiels de celle-ci ne doivent être imprimés ou autrement reproduits sans son autorisation.
In compliance with the Canadian Privacy Act some supporting forms may have been removed from this thesis.

While these forms may be included in the document page count, their removal does not represent any loss of content from the thesis.
Conformément à la loi canadienne sur la protection de la vie privée, quelques formulaires secondaires ont été enlevés de cette thèse.

Bien que ces formulaires aient inclus dans la pagination, il n'y aura aucun contenu manquant.

\section{Canada}




\section{Abstract}

This thesis presents a method for automatically minimizing the time required to obtain a single range image while providing the operator with a variety of important information about each measurement. Eight quality metrics are derived and used to adapt the scanning process in response to what is learned about the environment through the measurements, reducing the skill level required by an operator to perform basic 3D data acquisition using a laser range scanner. Of these eight quality metrics, six (outlier, resolvability, planarity, integration, aliasing, and enclosed) are new metrics and two (orientation and reflectivity) are improved versions of contemporary quality metrics.

A low-density raster scan is initially performed to obtain information, represented by quality metrics, about the field of view from the perspective of the laser range scanner. These metrics are initially used to interactively assist the operator in finding an alternative position for the scanner that should result in better quality measurements, if one exists locally. Experiments indicate that a locally optimal scanner viewpoint can typically be achieved in less than four iterations.

Once a locally optimal scanner viewpoint has been achieved, quality metrics are used to automatically divide the total field of view into three regions: likely to generate useful measurements, likely to generate redundant measurements, and likely to generate measurements that will be discarded. A series of high-density raster scans is then generated to maximize coverage of the region likely to generate useful measurements and minimize 
coverage of the region likely to generate measurements that will be discarded. The density of each subscan is automatically selected to minimize the likelihood of aliasing to a pre-defined target surface resolution. The method adapts the degree of overlap among low-density raster scans to maximize coverage while minimizing total scanning time, as well as adapts scan density to minimize observed aliasing to a pre-defined target surface resolution. Experimental results show that the total scanning time, excluding processing time, is significantly reduced compared to conventional methods.

All raster scans are merged to generate a composite range image using a qualityweighted maximum likelihood estimator. This method for generating range images can be combined with existing view planning procedures by using measurement quality information embedded in the composite range image. A new quality metric, repeatability, is derived to represent how consistently a measurement is obtained from a surface region. Quality metrics previously defined only for raster scans are then generalized for Delaunay mesh surfaces. 


\section{Acknowledgements}

Research leading to solutions to real-world problems does not take place in isolation; rather, it builds on the work of others. I would first like to acknowledge the work upon which my own research has been built, both those referenced in this work as well as countless others on whom their work was founded. We all, together, are working to build a mountain of knowledge, extending what is known about the world and what can be done with it. I only hope that my own contribution will have added even one more stone to this edifice.

Of those researchers on whom I have depended for insight and understanding, many could be found at the National Research Council of Canada's (NRC's) Visual Information Technology Group. I would like to acknowledge the support of the NRC from my early days as a student to my Master's and finally to the end of my Ph.D. They have provided funding variously in the form of student employment, conference travel and registration, and finally in the form of a Graduate Student Scholarship Supplement. I would in particular like to thank Francois Blais who has stood by me through seven years of educational advancement and provided me with research space, equipment, and computational resources. Much of my work would not have been possible without this assistance.

In parallel with the assistance provided by the $\mathrm{NRC}$, Carleton University, and in particular Dr. Victor Aitken, have guided me through the sometimes maze-like process 
that is our post-graduate educational experience. He, along with the staff of the Systems and Computer Engineering department, have always been there to help guide me through the bumps and rough patches of higher learning.

Finally, I would not be where I am today without the continued love and encouragement of my wife, Coralie, who provided endless moral support. It is to her that I dedicate this work. 


\section{Contents}

1 Introduction 1

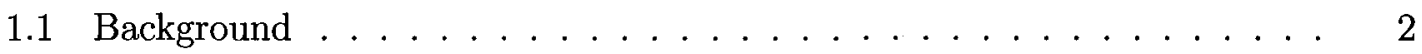

1.2 What is Quality? . . . . . . . . . . . . . . 4

1.3 Quality-based Model Acquisition ................ 5

1.3.1 Measurement Uncertainty . . . . . . . . . . . . . . 7

1.3.2 Measurement Resolution . . . . . . . . . . . . . . . . 10

1.4 Summary .............................. 11

2 Survey of Quality Metrics 12

2.1 Introduction . . . . . . . . . . . . . . . . . . . 12

2.2 Spatial Resolution . . . . . . . . . . . . . . . . . 14

2.3 Measurement Uncertainty-based Metrics . . . . . . . . . . . 15

2.3 .1 Measurement Uncertainty . . . . . . . . . . . . . 17

2.3 .2 Positional Uncertainty . . . . . . . . . . . . . . . . . 18

2.3 .3 Radial Uncertainty . . . . . . . . . . . . . . . . . 18

2.3.4 Environmental Effects . . . . . . . . . . . . . . 22

2.3.5 Measurement Uncertainty as a Quality Metric . . . . . . . . . 27

2.4 Signal Intensity-based Metrics . . . . . . . . . . . . . . . . . . . . . 29

2.4 .1 Intensity as a Quality Attribute . . . . . . . . . . . . . 33 


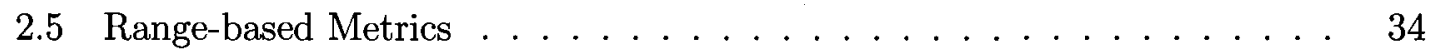

2.6 Neighbourhood-based Metrics . . . . . . . . . . . . . . . . 36

2.6 .1 Distance Metrics . . . . . . . . . . . . . . . 37

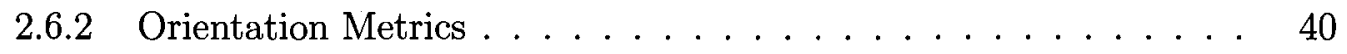

2.7 Total Quality Metric . . . . . . . . . . . . . . . 45

2.8 Unresolved Quality Issues $\ldots \ldots \ldots \ldots \ldots \ldots$

2.9 Summary $\ldots \ldots \ldots \ldots \ldots \ldots \ldots \ldots \ldots \ldots \ldots \ldots$

3 Region Mapping $\quad 49$

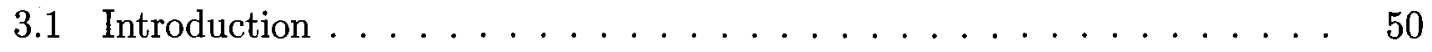

3.1 .1 Quality Metrics . . . . . . . . . . . . . . 50

3.2 Laser Range Scanner System . . . . . . . . . . . . . . . . . . . 52

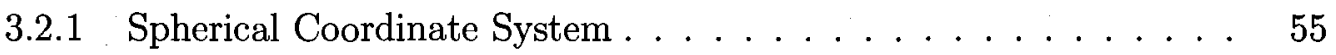

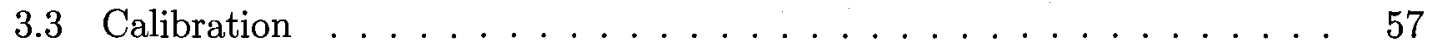

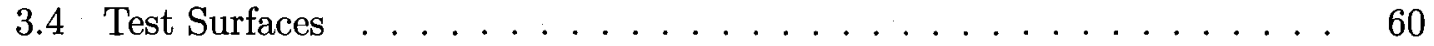

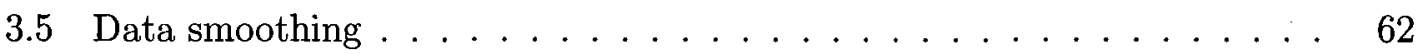

3.5 .1 Median Filtering $\ldots \ldots \ldots \ldots \ldots$

3.5.2 Spot Size-dependant Median Filtering . . . . . . . . . . . . 64

3.5 .3 Outlier Quality Metric . . . . . . . . . . . . . . . 68

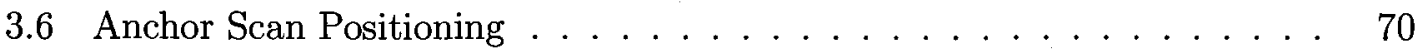

3.6.1 Orientation Quality Metric . . . . . . . . . . . . . 71

3.6.2 Resolvability Quality Metric . . . . . . . . . . . . . . 72

3.6.3 Quality-weighted Spot Size . . . . . . . . . . . . . . . . 76

3.6.4 Scanner Positioning . . . . . . . . . . . . . . . . . . 77

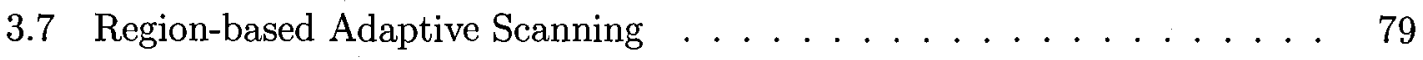

3.7.1 Planar Quality Metric . . . . . . . . . . . . . . . 79

3.7 .2 Reflectivity Quality Metric . . . . . . . . . . . . . 81 
3.7.3 Reflectivity-based Edges . . . . . . . . . . . . . . . . 83

3.7.4 Low-Outlier Detection . . . . . . . . . . . . . . . . . 83

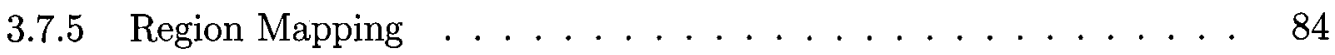

3.8 Subscan Generation . . . . . . . . . . . . . . . . . 85

3.9 Summary $\ldots \ldots \ldots \ldots \ldots \ldots \ldots$

4 Building the Composite Scan $\quad 90$

4.1 Introduction . . . . . . . . . . . . . . . . . . . . 91

4.2 Total Quality . . . . . . . . . . . . . . . . . 92

$4.2 .1 \quad$ Aliasing Quality Metric . . . . . . . . . . . . . . 92

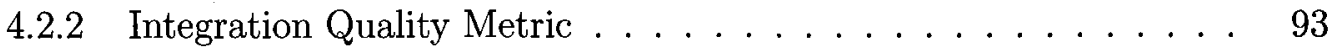

4.2 .3 Enclosed Quality Metric . . . . . . . . . . . . . . 95

4.2.4 Within-scan Total Quality Metric . . . . . . . . . . . . 96

4.3 Region Coverage . . . . . . . . . . . . . . . . . . . . . 98

4.3 .1 Rescan Region Coverage . . . . . . . . . . . . . . . . . 98

4.3 .2 Subscan Overlap . . . . . . . . . . . . . . . . . . . . . 99

4.3.3 Anti-aliasing Coverage . . . . . . . . . . . . . . . . . . . 102

4.3.4 Combining Overlap and Anti-aliasing . . . . . . . . . . . . 103

4.4 Composite Range Image Generation . . . . . . . . . . . . . . . . . . . 104

4.4 .1 Initialization Procedure . . . . . . . . . . . . . . . . . 105

4.4 .2 Update Procedure . . . . . . . . . . . . . . . . . 106

4.4.3 Option 4: Quality-weighted MKMV . . . . . . . . . . . . . 111

4.4.4 Non-return Measurements . . . . . . . . . . . . . . . . . 113

4.4.5 Repeatability Quality Metric . . . . . . . . . . . . . . . 114

4.4 .6 Results . . . . . . . . . . . . . . . . . . . 115

4.5 Within-composite Quality Metrics . . . . . . . . . . . . . 116

4.5 .1 Delaunay Neighbourhood . . . . . . . . . . . . . . . 116 
4.5.2 Composite Smoothing . . . . . . . . . . . . . 117

4.5.3 Within-composite Reflectivity Quality Metric . . . . . . . . 119

4.5.4 Within-composite Orientation Quality Metric . . . . . . . . 119

4.5.5 Within-composite Aliasing Quality Metric . . . . . . . . . . 120

4.5.6 Within-composite Total Quality Metric . . . . . . . . . . . . . . 120

4.6 Summary . . . . . . . . . . . . . . . . . . . 120

5 Conclusions and Future Work $\quad 123$

5.1 Summary and Conclusions . . . . . . . . . . . . . . 123

5.1 .1 Contributions and Innovations . . . . . . . . . . . . . . . 123

5.1 .2 Quality Metrics .................. 125

5.1 .3 Adaptive Scanning . . . . . . . . . . . . . . . . . 128

5.1 .4 Quality-based Merging . . . . . . . . . . . . . . . 132

5.2 Future Work . . . . . . . . . . . . . . . . . . 133

5.2 .1 Motion Distortion . . . . . . . . . . . . . . . 133

5.2 .2 Merged Measurement Map . . . . . . . . . . . . 135

5.2 .3 Registration Error . . . . . . . . . . . . . . 135

5.2 .4 Edge Distortion . . . . . . . . . . . . . . 136

5.2 .5 View Planning . . . . . . . . . . . . . . 137

$\begin{array}{lr}\text { References } & 140\end{array}$

$\begin{array}{ll}\text { Appendix A: Calibration } & 167\end{array}$

$\begin{array}{ll}\text { Appendix B: Test Surfaces } & 171\end{array}$

$\begin{array}{lr}\text { Appendix C: Filter Calibration } & 175\end{array}$

$\begin{array}{lr}\text { Appendix D: Outlier Metric } & 178\end{array}$ 
Appendix E: Orientation and Resolvability Metrics

Appendix F: Beam-Surface Intersection

Appendix G: Scanner Positioning

Appendix H: Reflectivity and Planarity Quality Maps

Appendix I: Regions Maps and Subscans

Appendix J: Aliasing, Integration, Completness and Total Quality Maps 207

Appendix K: Subscan Quality

Appendix L: Subscan Coverage

Appendix M: Scalar Equivalent Variance

M.1 Distance in Cartesian and Spherical Coordinates . . . . . . . . . . . 225

M.2 Mahalanobis Distance . . . . . . . . . . . . . . . . 227

M.3 Scalar Equivalent Variance . . . . . . . . . . . . . . . . . 228

$\begin{array}{ll}\text { Appendix N: Subscan Merger } & 231\end{array}$

Appendix O: Within-composite Quality Metrics $\quad 237$ 


\section{List of Figures}

1.1 Measurement accuracy versus precision . . . . . . . . . . 7

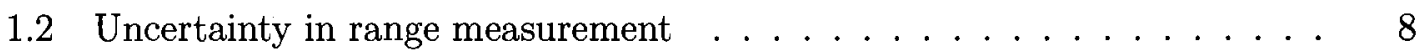

1.3 Range measurement accuracy . . . . . . . . . . . . . . . . . 9

1.4 Measurement accuracy versus height $\ldots \ldots \ldots \ldots$

2.1 Fixed-viewpoint laser range scanner . . . . . . . . . . . . . . . . . 16

2.2 Common range uncertainties . . . . . . . . . . . . . . . 20

2.3 Source of speckle noise . . . . . . . . . . . . . . 21

2.4 Speckle noise reduction . . . . . . . . . . . . . . . 22

2.5 Range discontinuity . . . . . . . . . . . . . . . . . 24

2.6 Surface penetration error . . . . . . . . . . . . . . . . . 25

2.7 Reflectivity discontinuity . . . . . . . . . . . . . 26

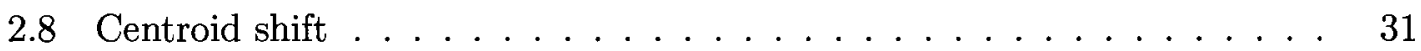

2.9 Laser profile . . . . . . . . . . . . . . . . . . . . . . 35

2.10 Facet elongation . . . . . . . . . . . . . . . . . . . 43

2.11 Limits of facet elongation . . . . . . . . . . . . . . 44

3.1 Laser range scanner . . . . . . . . . . . . . . . . . . . . . . 53

3.2 Sensor uncertainty orientation . . . . . . . . . . . 56

3.3 Range and intensity standard deviation, and spot radius . . . . . . . 59 
$3.4 \theta$ standard deviation and rotational resolution $\ldots \ldots \ldots \ldots$

3.5 Residual-minimizing median window size . . . . . . . . . . . . 65

3.6 Residual-minimizing median window repetitions . . . . . . . . . 65

3.7 Range uncertainty versus inter-measurement distance . . . . . . . . . 67

3.8 Beam intersection with angled planar surface . . . . . . . . 73

3.9 Experiment \#1 reposition $3 \ldots \ldots \ldots \ldots$

3.10 Experiment \#4 repositioning . . . . . . . . . . . . . . . . . . 78

4.1 Integration distance $\ldots \ldots \ldots \ldots \ldots$

4.24 -element subscan overlap . . . . . . . . . . . . . . . . 101

4.37 -element subscan overlap . . . . . . . . . . . . . . . . 101

4.4 11-element subscan overlap . . . . . . . . . . . . . . . 101

4.5 Delaunay and 8 -neighbours . . . . . . . . . . . . 117

4.6 Experiment \#1 facet map . . . . . . . . . . . . . . . . . . . 118

4.7 Experiment \#2 facet map . . . . . . . . . . . . . . . . . 118

4.8 Experiment \#3 facet map . . . . . . . . . . . . . . . . . . . . 118

4.9 Experiment \#4 facet map . . . . . . . . . . . . . . . . . . . . 118

4.10 Experiment \#5 facet map . . . . . . . . . . . . . . . . . . . . . . . 118

5.1 Motion distortion . . . . . . . . . . . . . . . . 134

A-1 Data set \#1 . . . . . . . . . . . . . . . . . 167

A-2 Data set \#2 . . . . . . . . . . . . . . . . . 167

A-3 Data set \#3 . . . . . . . . . . . . . . . . . 168

A-4 Data set \#4 . . . . . . . . . . . . . . . . 168

A-5 Data set \#5 . . . . . . . . . . . . . . . . 168

A-6 Data set \#6 . . . . . . . . . . . . . . . . . . 168

A-7 Data set \#7 . . . . . . . . . . . . . . . . 169 
A-8 Data set \#8 . . . . . . . . . . . . . . . . . . . . . . . . . 169

A-9 Range uncertainty model . . . . . . . . . . . . . . . . . . . . . 169

A-10 Rotational uncertainty model . . . . . . . . . . . . . . . . . 169

A-11 Reference intensity model . . . . . . . . . . . . . . . . 170

A-12 Intensity uncertainty model $\ldots \ldots \ldots \ldots$

B-1 Experiment \#1: front view $\ldots \ldots \ldots \ldots \ldots \ldots \ldots \ldots$

B-2 Experiment \#1: side view . . . . . . . . . . . . . . . . 171

B-3 Experiment \#2: front view . . . . . . . . . . . . . . . 172

B-4 Experiment \#2: detail view $\ldots \ldots \ldots \ldots \ldots \ldots \ldots \ldots \ldots$

B-5 Experiment \#3: front view . . . . . . . . . . . . . . . . 172

B-6 Experiment \#3: detail view . . . . . . . . . . . . . . . . . 172

B-7 Experiment \#4: front view . . . . . . . . . . . . . . . . 173

B-8 Experiment \#4: side view $\ldots \ldots \ldots \ldots \ldots \ldots \ldots \ldots$

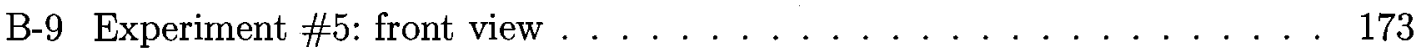

B-10 Experiment \#5: detail view $\ldots \ldots \ldots \ldots \ldots \ldots$

B-11 Experiment \#5: picture detail . . . . . . . . . . . . . . 174

B-12 Experiment \#5: planter detail . . . . . . . . . . . . . . 174

C-1 Scan Area/Spot Size $=0.003$. . . . . . . . . . . . . . . 175

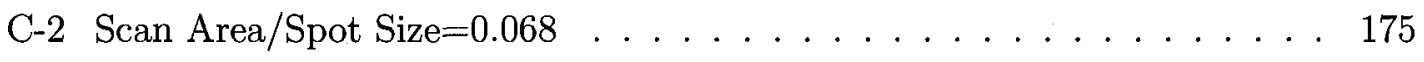

C-3 Scan Area/Spot Size $=0.279 \ldots \ldots \ldots \ldots \ldots$

C-4 Scan Area/Spot Size $=1.130 \ldots \ldots \ldots \ldots \ldots$

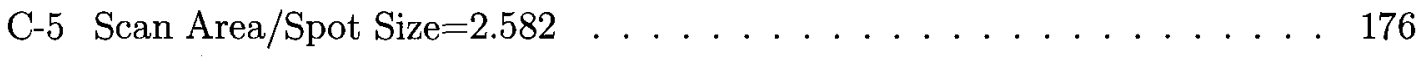

C-6 Scan Area/Spot Size $=4.702 \ldots \ldots \ldots \ldots \ldots$

C-7 Scan Area/Spot Size $=7.585 \ldots \ldots \ldots \ldots$. . . . . . . . . 177

C-8 Scan Area/Spot Size $=11.654 \ldots \ldots \ldots \ldots \ldots \ldots$. . . . . . . 177 


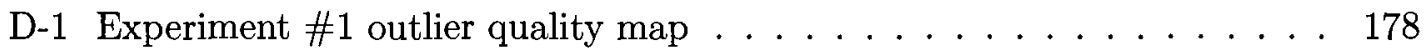

D-2 Experiment \#1 smoothed outlier map . . . . . . . . . . . . . 178

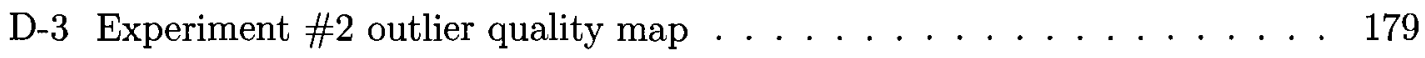

D-4 Experiment \#2 smoothed outlier map . . . . . . . . . . . . . 179

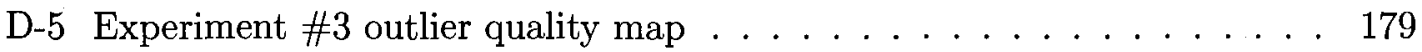

D-6 Experiment \#3 smoothed outlier map . . . . . . . . . . . . . . 179

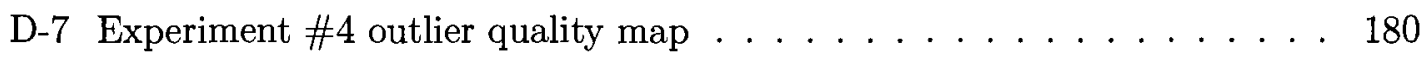

D-8 Experiment \#4 smoothed outlier map . . . . . . . . . . . . . . . 180

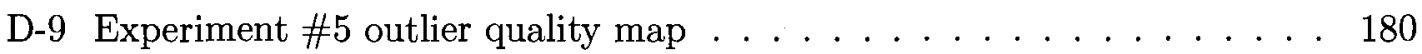

D-10 Experiment \#5 smoothed outlier map . . . . . . . . . . . 180

E-1 Experiment \#1 orientation quality map . . . . . . . . . . . . . . . 181

E-2 Experiment \#1 resolvability quality map . . . . . . . . . . . . . . 181

E-3 Experiment \#2 orientation quality map . . . . . . . . . . . . 182

E-4 Experiment \#2 resolvability quality map . . . . . . . . . . . . . . . 182

E-5 Experiment \#3 orientation quality map . . . . . . . . . . . . . . 182

E-6 Experiment \#3 resolvability quality map . . . . . . . . . . . . . . 182

E-7 Experiment \#4 orientation quality map . . . . . . . . . . . . 183

E-8 Experiment \#4 resolvability quality map . . . . . . . . . . . . . . 183

E-9 Experiment \#5 orientation quality map . . . . . . . . . . . . . . . 183

E-10 Experiment \#5 resolvability quality map . . . . . . . . . . . 183

F-1 Beam-surface intersection . . . . . . . . . . . . . . . 184

G-1 Experiment \#1 repositioning $1 \ldots \ldots \ldots \ldots \ldots \ldots$

G-2 Experiment \#1 repositioning $2 \ldots \ldots \ldots \ldots$

G-3 Experiment \#1 repositioning $3 \ldots \ldots \ldots$

G-4 Experiment \#3 repositioning $1 \ldots \ldots \ldots \ldots \ldots \ldots$ 
G-5 Experiment \#3 repositioning $2 \ldots \ldots$. . . . . . . . . . . . . 190

G-6 Experiment \#3 repositioning 3 . . . . . . . . . . . . . . . . . . . 190

G-7 Experiment \#4 repositioning $1 \ldots \ldots$. . . . . . . . . . . 191

G-8 Experiment \#4 repositioning $2 \ldots \ldots \ldots 1$

G-9 Experiment \#4 repositioning 3 . . . . . . . . . . . . . . . . . . 191

G-10 Experiment \#5 repositioning $1 \ldots \ldots$. . . . . . . . . . . . . 192

G-11 Experiment \#5 repositioning $2 \ldots \ldots$. . . . . . . . . . . 192

G-12 Experiment \#5 repositioning $3 \ldots \ldots$. . . . . . . . . . 192

H-1 Experiment \#1 pre-reflectivity planarity quality map . . . . . . . . . . 194

H-2 Experiment \#1 reflectivity quality map . . . . . . . . . . . . . . 194

H-3 Experiment \#1 post-reflectivity planarity quality map . . . . . . . . . . 194

H-4 Experiment \#1 post-outlier planarity quality map . . . . . . . . . . . . . 194

H-5 Experiment \#2 pre-reflectivity planarity quality map . . . . . . . . . . 195

H-6 Experiment \#2 reflectivity quality map . . . . . . . . . . . . . . . . 195

H-7 Experiment \#2 post-reflectivity planarity quality map . . . . . . . . . . 195

H-8 Experiment \#2 post-outlier planarity quality map . . . . . . . . . . . 195

H-9 Experiment \#3 pre-reflectivity planarity quality map . . . . . . . . . . 196

H-10 Experiment \#3 reflectivity quality map . . . . . . . . . . . . . . 196

H-11 Experiment \#3 post-reflectivity planarity quality map . . . . . . . . . 196

H-12 Experiment \#3 post-outlier planarity quality map . . . . . . . . . . 196

H-13 Experiment \#4 pre-reflectivity planarity quality map . . . . . . . . . . 197

H-14 Experiment \#4 reflectivity quality map . . . . . . . . . . . . . . . . 197

H-15 Experiment \#4 post-reflectivity planarity quality map . . . . . . . . . 197

H-16 Experiment \#4 post-outlier planarity quality map . . . . . . . . . . 197

H-17 Experiment \#5 pre-reflectivity planarity quality map . . . . . . . . . . 198

H-18 Experiment \#5 reflectivity quality map . . . . . . . . . . . . . . . 198 
H-19 Experiment \#5 post-reflectivity planarity quality map . . . . . . . . . . 198

H-20 Experiment \#5 post-outlier planarity quality map . . . . . . . . . . . 198

I-1 Experiment \#1 region map . . . . . . . . . . . . . . . . . 202

I-2 Experiment \#1 target-based subscan map . . . . . . . . . . . . . . . 202

I-3 Experiment \#1 spot-based subscan map . . . . . . . . . . . . . . . . 202

I-4 Experiment \#2 region map . . . . . . . . . . . . . . 203

I-5 Experiment \#2 target-based subscan map . . . . . . . . . . . . . 203

I-6 Experiment \#2 spot-based subscan map . . . . . . . . . . . . . . . . . . 203

I-7 Experiment \#3 region map . . . . . . . . . . . . . . . . . 204

I-8 Experiment \#3 target-based subscan map . . . . . . . . . . . . . 204

I-9 Experiment \#3 spot-based subscan map . . . . . . . . . . . . . . . . . . 204

I-10 Experiment \#4 region $\operatorname{map} \ldots \ldots \ldots \ldots$. . . . . . . . . . . . . .

I-11 Experiment \#4 target-based subscan map . . . . . . . . . . . . 205

I-12 Experiment \#4 spot-based subscan map . . . . . . . . . . . . . 205

I-13 Experiment \#5 region map . . . . . . . . . . . . . . . . 206

I-14 Experiment \#5 target-based subscan map . . . . . . . . . . . . 206

I-15 Experiment \#5 spot-based subscan map . . . . . . . . . . . . . . 206

J-1 Experiment \#1 aliasing quality map . . . . . . . . . . . . . . 208

$\mathrm{J}-2$ Experiment \#1 integration quality map . . . . . . . . . . . . 208

J-3 Experiment \#1 enclosed quality map . . . . . . . . . . . . . . 208

J-4 Experiment \#1 within-scan total quality map . . . . . . . . . . . 208

J-5 Experiment \#1 acceptability map . . . . . . . . . . . . . . 208

J-6 Experiment \#2 aliasing quality map . . . . . . . . . . . . . . . 209

J-7 Experiment \#2 integration quality map . . . . . . . . . . . . . . 209

J-8 Experiment \#2 enclosed quality map . . . . . . . . . . . . . . . . . . . . 209

J-9 Experiment \#2 within-scan total quality map . . . . . . . . . . . . 209 
J-10 Experiment \#2 acceptability map . . . . . . . . . . . . . . . . . 209

J-11 Experiment \#3 aliasing quality map . . . . . . . . . . . . . . . . 210

J-12 Experiment \#3 integration quality map . . . . . . . . . . . . . . . . . 210

J-13 Experiment \#3 enclosed quality map . . . . . . . . . . . . . . . . . 210

J-14 Experiment \#3 within-scan total quality map . . . . . . . . . . . . 210

J-15 Experiment \#3 acceptability map . . . . . . . . . . . . . . . . . 210

J-16 Experiment \#4 aliasing quality map . . . . . . . . . . . . . . . 211

J-17 Experiment \#4 integration quality map . . . . . . . . . . . . . . . 211

J-18 Experiment \#4 enclosed quality map . . . . . . . . . . . . . . . 211

J-19 Experiment \#4 within-scan total quality map . . . . . . . . . . . . 211

J-20 Experiment \#4 acceptability map . . . . . . . . . . . . . . . . . 211

J-21 Experiment \#5 aliasing quality map . . . . . . . . . . . . . . . . 212

J-22 Experiment \#5 integration quality map . . . . . . . . . . . . . . . . 212

J-23 Experiment \#5 enclosed quality map . . . . . . . . . . . . . 212

J-24 Experiment \#5 within-scan total quality map . . . . . . . . . . . . 212

J-25 Experiment \#5 acceptability map . . . . . . . . . . . . . . . . . 212

K-1 Experiment \#1 subscan in anchor . . . . . . . . . . . . . . . 214

K-2 Experiment \#1 subscan map . . . . . . . . . . . . . . . . . 214

K-3 Experiment \#1 subscan aliasing quality map . . . . . . . . . . . . . 214

K-4 Experiment \#1 subscan integration quality map . . . . . . . . . . . 214

K-5 Experiment \#1 subscan total quality map . . . . . . . . . . . . . . . 214

K-6 Experiment \#1 subscan acceptability map . . . . . . . . . . . . . . . . . 214

K-7 Experiment \#2 subscan in anchor . . . . . . . . . . . . . . . . . 215

K-8 Experiment \#2 subscan map . . . . . . . . . . . . . . . . . . 215

K-9 Experiment \#2 subscan aliasing quality map . . . . . . . . . . . . . . 215

K-10 Experiment \#2 subscan integration quality map . . . . . . . . . . . 215 
K-11 Experiment \#2 subscan total quality map $\ldots \ldots \ldots \ldots \ldots$

K-12 Experiment \#2 subscan acceptability map . . . . . . . . . . . . 215

K-13 Experiment \#3 subscan in anchor . . . . . . . . . . . . 216

K-14 Experiment \#3 subscan map . . . . . . . . . . . . . . . 216

K-15 Experiment \#3 subscan aliasing quality map . . . . . . . . . . . . 216

K-16 Experiment \#3 subscan integration quality map . . . . . . . . . 216

K-17 Experiment \#3 subscan total quality map . . . . . . . . . . . 216

K-18 Experiment \#3 subscan acceptability map . . . . . . . . . . . . 216

K-19 Experiment \#4 subscan in anchor . . . . . . . . . . . . . . . . . 217

K-20 Experiment \#4 subscan map . . . . . . . . . . . . . . . . . 217

K-21 Experiment \#4 subscan aliasing quality map . . . . . . . . . . 217

K-22 Experiment \#4 subscan integration quality map . . . . . . . . . 217

K-23 Experiment \#4 subscan total quality map . . . . . . . . . . . . 217

K-24 Experiment \#4 subscan acceptability map . . . . . . . . . . . 217

K-25 Experiment \#5 subscan in anchor . . . . . . . . . . . . 218

K-26 Experiment \#5 subscan map . . . . . . . . . . . . . . . 218

K-27 Experiment \#5 subscan aliasing quality map . . . . . . . . . . . . . 218

K-28 Experiment \#5 subscan integration quality map . . . . . . . . . 218

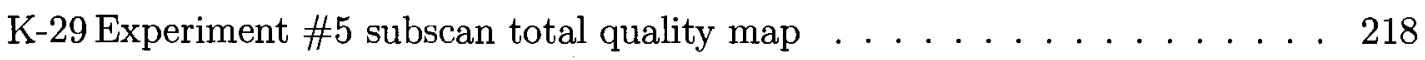

K-30 Experiment \#5 subscan acceptability map $\ldots \ldots \ldots \ldots \ldots \ldots . . \ldots 218$

L-1 Experiment \#1 subscan coverage $\ldots \ldots \ldots \ldots \ldots \ldots$

L-2 Experiment \#1 Unscanned region . . . . . . . . . . . . . . . 220

L-3 Experiment \#1 second pass subscans . . . . . . . . . . . . . 220

L-4 Experiment \#2 subscan coverage $\ldots \ldots \ldots \ldots \ldots \ldots \ldots \ldots . \ldots . \ldots . \ldots 221$

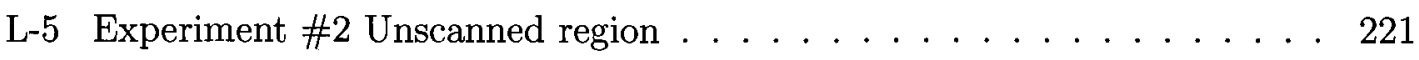

L-6 Experiment \#2 second pass subscans $\ldots \ldots \ldots \ldots \ldots \ldots \ldots \ldots$ 
L-7 Experiment \#3 subscan coverage . . . . . . . . . . . . . . . . . 222

L-8 Experiment \#3 Unscanned region . . . . . . . . . . . . . . . . 222

L-9 Experiment \#3 second pass subscans . . . . . . . . . . . . . . . 222

L-10 Experiment \#4 subscan coverage . . . . . . . . . . . . . . . 223

L-11 Experiment \#4 Unscanned region . . . . . . . . . . . . . . . . 223

L-12 Experiment \#4 second pass subscans . . . . . . . . . . . . . 223

L-13 Experiment \#5 subscan coverage . . . . . . . . . . . . . . . . . 224

L-14 Experiment \#5 Unscanned region . . . . . . . . . . . . . . . . . 224

L-15 Experiment \#5 second pass subscans . . . . . . . . . . . . . . . . 224

M-1 Scalar Equivalent Variance . . . . . . . . . . . . . . . . . . 229

N-1 Experiment \#1 subscans . . . . . . . . . . . . . . . . . . . . 232

N-2 Experiment \#1 subscans close-up . . . . . . . . . . . . . . . . . . 232

N-3 Experiment \#1 repeatability quality map . . . . . . . . . . . . . . . . . 232

N-4 Experiment \#1 within-scan total quality map . . . . . . . . . . . . 232

$\mathrm{N}-5$ Experiment \#2 subscans . . . . . . . . . . . . . . . . 233

N-6 Experiment \#2 subscans close-up . . . . . . . . . . . . . . . 233

N-7 Experiment \#2 repeatability quality map . . . . . . . . . . . . 233

N-8 Experiment \#2 within-scan total quality map . . . . . . . . . 233

N-9 Experiment \#3 subscans . . . . . . . . . . . . . . . . . 234

N-10 Experiment \#3 subscans close-up . . . . . . . . . . . . . . . 234

N-11 Experiment \#3 repeatability quality map . . . . . . . . . . . . 234

N-12 Experiment \#3 within-scan total quality map . . . . . . . . . . . . 234

N-13 Experiment \#4 subscans . . . . . . . . . . . . . . . . . . . 235

N-14 Experiment \#4 subscans close-up . . . . . . . . . . . . . . 235

N-15 Experiment \#4 repeatability quality map . . . . . . . . . . . . . 235

N-16 Experiment \#4 within-scan total quality map . . . . . . . . . . . 235

$\operatorname{xix}$ 
N-17 Experiment \#5 subscans . . . . . . . . . . . . . . . . . 236

N-18 Experiment \#5 subscans close-up . . . . . . . . . . . . . . 236

N-19 Experiment \#5 repeatability quality map . . . . . . . . . . . . 236

N-20 Experiment \#5 within-scan total quality map . . . . . . . . . . 236

O-1 Experiment $\# 1$ reflectivity quality map . . . . . . . . . . . . . 238

O-2 Experiment $\# 1$ orientation quality map . . . . . . . . . . . . 238

O-3 Experiment \#1 aliasing quality map . . . . . . . . . . . . . 238

O-4 Experiment \#1 within-composite total quality map . . . . . . . . . . 238

O-5 Experiment \#2 reflectivity quality map . . . . . . . . . . . . . 239

O-6 Experiment \#2 orientation quality map . . . . . . . . . . . . 239

O-7 Experiment \#2 aliasing quality map . . . . . . . . . . . . 239

O-8 Experiment \#2 within-composite total quality map . . . . . . . . . . . 239

O-9 Experiment \#3 reflectivity quality map . . . . . . . . . . . . . . . 240

O-10 Experiment \#3 orientation quality map . . . . . . . . . . . . . . 240

O-11 Experiment \#3 aliasing quality map . . . . . . . . . . . . . . . 240

O-12 Experiment \#3 within-composite total quality map . . . . . . . . . . 240

O-13 Experiment \#4 reflectivity quality map . . . . . . . . . . . . . . 241

O-14 Experiment \#4 orientation quality map . . . . . . . . . . . . . . 241

O-15 Experiment \#4 aliasing quality map . . . . . . . . . . . . . . . 241

O-16 Experiment \#4 within-composite total quality map . . . . . . . . . . 241

O-17 Experiment \#5 reflectivity quality map . . . . . . . . . . . . . . . 242

O-18 Experiment \#5 orientation quality map . . . . . . . . . . . . . 242

O-19 Experiment \#5 aliasing quality map . . . . . . . . . . . . . . 242

O-20 Experiment \#5 within-composite total quality map . . . . . . . . . . 242 


\section{List of Tables}

2.1 Environmental factors affecting measurement uncertainty . . . . . . . . 23

2.2 Environmental factors affecting return signal intensity . . . . . . . . . . 32

4.1 Subscan overlap coverage . . . . . . . . . . . . . . . 100

4.2 Subscan efficiency with overlap . . . . . . . . . . . 100

4.3 Subscan anti-aliasing . . . . . . . . . . . . . . . 103

4.4 Subscans efficiency with anti-aliasing . . . . . . . . . . . . 103

4.5 Combined anti-aliasing and overlap . . . . . . . . . . . . 104

4.6 Combined anti-aliasing and overlap efficiency . . . . . . . . . . 105

G-1 Experiment \#1 scanner placement . . . . . . . . . . . . . . . 187

G-2 Experiment \#2 scanner placement . . . . . . . . . . . . . . . 187

G-3 Experiment \#3 scanner placement . . . . . . . . . . . . . . . 187

G-4 Experiment \#4 scanner placement . . . . . . . . . . . . . . . . . . 188

G-5 Experiment \#5 scanner placement . . . . . . . . . . . . 188

I-1 Full and reduced scans $\ldots \ldots \ldots \ldots \ldots$

I-2 Target-based Subscan efficiency . . . . . . . . . . . . . . . 200

I-3 Spot-based Subscan efficiency . . . . . . . . . . . . . . . . . 201

L-1 Second pass subscan efficiency . . . . . . . . . . . . . . . . . 219

$\mathrm{xxi}$ 


\section{Chapter 1}

\section{Introduction}

Currently absent in the field of medium- to large-volume scanning is an interactive system capable of obtaining a complete high-resolution model of a scene or object in situ by a minimally-trained operator. Some attempts have been made, most notably the work of Sequeira et al. [1]-[4] and Blais et al. [5]. Prior to constructing such a system, many issues need to be addressed including automatic image registration, view planning, metrics to assess model and measurement quality, ways to minimize the number of measurements obtained, and ways to speed the measurement process. One can envision a system that would utilize a flying-point laser scanner and would iteratively direct the operator in where to position the scanner to ensure that the model coverage was as complete as possible. Such a system would use off-the-shelf components to minimize the cost of construction, and would be fast enough to allow the operator to obtain a complete model in one or two sessions [6]. The system would then complete the scan of the environment in a way that maximizes model fidelity but minimizes the number of samples obtained.

The goal of this study was not to develop a complete system; rather, it focused on how to obtain the initial, or anchor [7] [8], model. This study is a proof-of-concept that examines how measurement quality could be used to drive a more adaptive approach 
to surface scanning. It is restricted to the case of a scanner placd in a static, stable position during each scan to obtained measurements from a static scene. A low-density raster scan is initially performed to generate an approximate model and then a series of high-density raster scans are performed to augment the model. A projected spherical Delaunay surface, referred to as a region map, is constructed and divided into three regions. These regions indicate where high-density scanning should yield measurements that will improve the quality of the model, where additional measurements would most likely be redundant, and where additional measurements would most likely be discarded as being of insufficient quality.

Chapter 2 presents a survey of how laser range scanner measurement quality has been previously assessed. Chapter 3 introduces several new ways to assess measurement quality, referred to as quality metrics, within the context of a data acquisition system that uses model quality to minimize the number of measurements obtained while automatically scanning a surface. In Chapter 4 , the anchor and subscan measurements are evaluated using several new quality metrics before being merged into a range image that combines all measurements obtained from the current viewpoint. The results and conclusions arising from the experiments performed during the course of this study are summarized in Chapter 5.

\subsection{Background}

Medium- and large-volume models may be acquired for robot vision [9]-[11] and control [12]-[16], inspection [15] [17]-[20], geological surveying [17] [21]-[23], urban planning [24] [25], virtual reality [17] [26]-[29], or heritage documentation [6]-[8] [30]-[39]. In such cases, it is the topology of the surface being scanned that is of primary importance so image-based techniques are generally insufficient [31]. In surveying and inspection applications, a geometric representation of an object or scene may be all that is required [17]; 
however, generating such models can result in large data sets so the challenge is in how to minimize the number of data points while minimizing the potential loss of valuable information [40]. In this study, a multi-stage scanning process was developed, and verified through experimentation, to significantly reduce the number of useful measurements obtained from a single viewpoint.

An issue in acquiring high-quality measurements for object or scene virtualization is the need for a skilled operator [31]. Problems such as measurement errors and view planning typically require the intervention of a person with expertise, not only with the equipment but in the data processing and rendering aspects as well. For example, an unskilled operator may not be aware that abrupt changes in surface structure can introduce artefacts that can significantly reduce the quality of the model [33] [41]. The greater the required skill level of the operator the fewer candidates are available and, consequently, the greater the cost of acquiring the services of that person. In order to confidently reduce the skill level of the operator, many error-checking and correcting procedures need to be automated. In this study, quality metrics are developed and used to partially automate the task of both selecting a locally optimal scanner viewpoint and orientation, and to direct the scanning process from that viewpoint. This reduces the skill level required of the operator and could ultimately reduce the cost of acquiring high-quality models. Moreover, the wide variety of quality metrics developed in this study provide the operator with a wealth of information about the quality of the measurements in particular, as well as the model in general, to aid the operator in planning the acquisition strategy.

Often what is being modeled cannot be brought into a controlled environment but must be handled in situ. In many of these cases access to the objects being modeled may also be limited [6] [30]. Historical or archaeological sites and structures are withdrawn from public display during recording, the daily operation of buildings and other structures may be suspended or restricted while scans are performed, and equipment being scanned 
must often be taken out of operation. In some cases the object being scanned is slated for destruction or will be changed substantially soon after the scanning process is complete, making it important that the data be acquired accurately the first time as well as quickly. According to Beraldin et al. [34], the speed of acquiring a complete model is primarily limited by the time required to determine the next best view; however, the accuracy of global models obtained by merging local models from different viewpoints is limited by the fidelity of each of the local models. In this study, a scanning process is developed, and experimentally verified, to significantly reduce the total scanning time from a single scanner viewpoint. By reducing the within-viewpoint scanning time, the total acquisition time for a model is also reduced.

\subsection{What is Quality?}

The term "quality" has multiple definitions but from the perspective of quality control the term quality can be defined as the degree to which one or more inherent characteristics fulfill stated requirements. With respect to laser range scanner data, a common requirement is for the resulting model to closely match ground truth; however, ground truth data is generally only available during either calibration or testing. Within the context of this study, ground truth comparison is not useful for adapting the scanning process in response to environmental conditions because ground truth data is rarely available in a real-world situation.

A useful set of requirements for adaptive scanning is the degree to which different measurement attributes either meet pre-defined thresholds or match the ideal conditions under which the resolution and uncertainty models were obtained. Attributes that can be compared to pre-defined thresholds are referred to as exclusive attributes because measurements with attributes that exceed the threshold values are considered unacceptable 
for use in the final model. Attributes that represent a deviation from the calibration environment are referred to as augmentative attributes.

\subsection{Quality-based Model Acquisition}

A significant issue in building virtual models of real scenes and objects is that insufficiencies in the model are often discovered only after data processing has been completed. This necessitates a return to the site of the object; something that can be time-consuming and potentially expensive. Scott [42] recognized the importance of integrating model quality into the scanning process and proposed a two-stage approach to model acquisition; however, his work was confined to small-volume scanning in which the position of the scanner and target could be tightly controlled. In this study, a quality-based approach is applied to medium volume scanning, but can be extended to either large- or small-volume situations.

Several researchers have used model quality as a prerequisite to view planning [2] [3] [43]. These researchers defined model quality as a measure of the degree of confidence one has in the fidelity of the model in comparison to some agreed-upon criteria. Model quality depends upon many factors that include:

- measurement uncertainty: the level of uncertainty associated with each measurement in a particular scan;

- model quality: the probability that there are no more features smaller than the minimum resolution;

- model coverage: the size and number of holes as a fraction of the total surface covered, where holes represent insufficient data to model a region;

- motion distortion: the level of inter-scan error; and 
- registration error: the level of inter-viewpoint error.

In this study, motion distortion and registration error are not considered; these issues can be addressed as part of future research. The approach developed in this study assumes that no prior information about the environment being scanned is available. A raster scan is performed to initialize the model, and this model is then used to formulate assumptions about the environment that can then be tested using subsequent scans. The problem of model coverage is addressed by assuming the measurements obtained thus far can be used to generate a watertight surface model that represents a best guess representation of the scene being scanned.

As with any prediction-based method, the approach developed for this dissertation makes certain trade-offs. The region-based approach involves deciding on which portions of the rescan map should be rescanned using high-density raster scans and which can safely be ignored. When a region is ignored based on a quality analysis of the anchor scan, it is assumed that sufficient information has been obtained such that the ignored region would yield no new useful information. The only way to be certain that all useful information has been obtained is to use a complete high-density scan of the environment; however, this approach is time-consuming and can result in many measurements that are eventually discarded. As a result, the cost of using a prediction-based method is the chance that important information about the surface may not be obtained. The approach described in this dissertation assumes that the risk of missing important information is low; however, this assumption is highly situationally dependant. If model fidelity is critical, then the approach used in this dissertation should be used with caution; however, this method is ideally suited to more general-purpose situations where cost and time are more important than the risk of missing fine model details. 


\subsubsection{Measurement Uncertainty}

Laser range scanners do not return exactly the point of intersection of a laser with a surface in the environment; rather, they return an estimate of the point of intersection. Each measurement has associated with it a degree of uncertainty, often referred to as the accuracy of the measurement; however, accuracy defines how well the measurement agrees with the ground truth measurement [30] [44] [45] while uncertainty defines how much a series of measurements arising from a given intersection point vary [30] [44]. If a scanner is well calibrated then the arithmetic mean of a series of measurements obtained for a given intersection point should be a close approximation of the intersection point. In that case, the spread, or uncertainty, of the measurements should give an indication of the expected accuracy of the sensor. A term related to uncertainty is precision which is the degree of agreement among independent measurements [44] [45]. The relationship between precision and accuracy is shown in Figure 1.1.

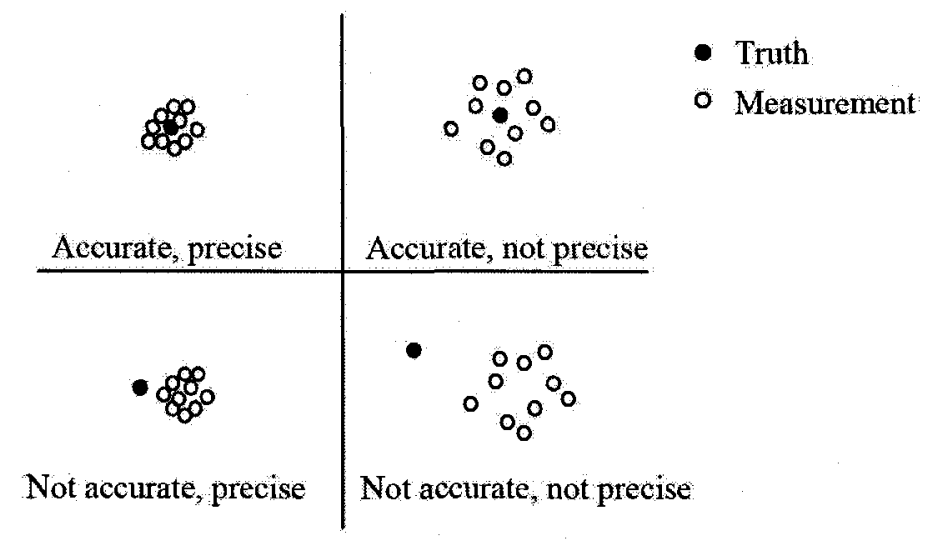

Figure 1.1: A measurement can be either accurate, or precise, or both. An accurate measurement is close to the ground truth measurement (top row) while precise measurements are tightly clustered (left side) (Reproduced from Figure 3 of [45]). 
Sensor uncertainty models for laser range scanners consist of each measurement being visualized as a probability field [46] in that the measurement uncertainty represents the probability that the surface is close to the measurement. Figure 1.2 provides a graphical interpretation of the uncertainty associated with a range measurement. The uncertainty in how well the measurement point approximates the intersection point increases as the volume scanned increases. Even in systems, such as time-of-flight scanners, in which the range error is relatively constant between the minimum and maximum effective range, lateral errors increase linearly with an increase in range. Figure 1.3 shows an example of this applied to a section of wall from a Byzantine crypt. If terrestrial-based scanning is used for large structures such as a cathedral, there may be a significant difference among the measurement errors associated with each measurement point [8] as can be seen in Figure 1.4. Moreover, triangulation scanners exhibit range errors that increase with the square of the range [15] [33] [41] [47].

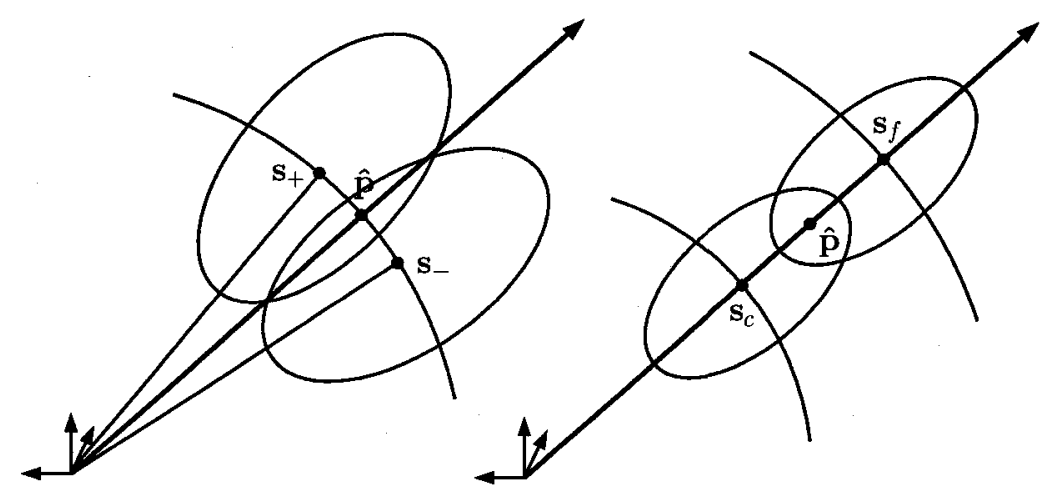

Figure 1.2: A measurement $\hat{\mathbf{p}}$ is an estimate of the location of the intersection of a laser with a surface. The measurement could be as close to the origin as $\mathbf{s}_{c}$ or as far away from the origin as $\mathbf{s}_{f}$. Similarly, the true intersection point could rotationally be as far from the measurement point as either $\mathbf{s}_{+}$or $\mathbf{s}_{-}$. 


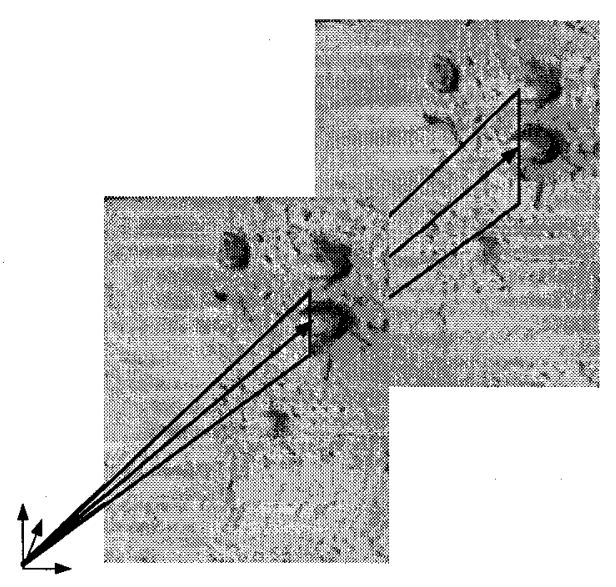

Figure 1.3: For a given level of rotational measurement uncertainty, increasing the distance between the scanner and the surface results in an increase in the lateral measurement uncertainty (Modified from Figure $5 \mathrm{a}$ of [31]).

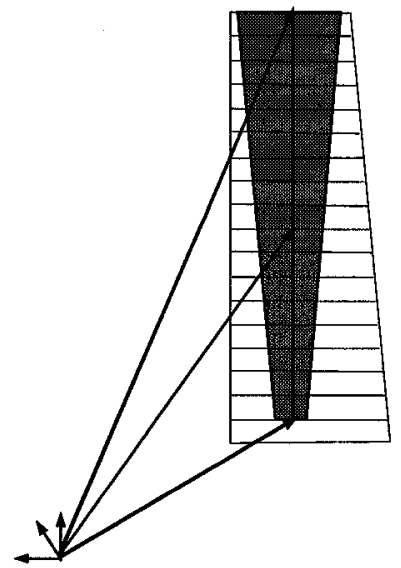

Figure 1.4: Assuming the scanner position is limited to within a metre or two of ground level, the lateral measurement uncertainty increases as the height of the structure increases due to the increase in the distance between the scanner and the surface. 
Measurement uncertainty is generally modeled with respect to some standard situation as part of a calibration process. In Chapter 2, the various factors that can cause true measurement uncertainty to vary from model-based uncertainty are explored, as are the ways in which researchers have attempted to quantify these factors. Quality metrics are used to represent the degree of confidence one has in how closely some aspect the real measurement situation mirrors the situation used during calibration. In Chapter 3 and Chapter 4 , these factors are quantified in a standardized manner.

\subsubsection{Measurement Resolution}

The resolution of a laser range scanner measurement is dependant on the size of the laser spot that illuminates the surface at the point the measurement is obtained, defined by the Rayleigh criterion [48], and represents the size of the smallest feature that can be resolved [49] [50]. Features within an illuminated region contribute to the return signal intensity which is used to approximate the intersection point of the laser with the surface [51]. Assuming a circular beam cross-section, the laser spot area should form a circle; however, environmental factors such as spatial discontinuities [52] and surface orientation can change the shape of the illuminated region. In this study, the shape of the laser spot in the mid-field region is derived for an arbitrary surface orientation, and is used to create quality metrics based on the resolution of the laser range scanner. Moreover, these quality metrics combine laser range scanner resolution with model-based measurement uncertainty rather than addressing them separately. Laser range scanner resolution is generally modelled under the assumption that the illuminated region is stationary at the time the measurement is acquired. In this study, a quality metric is presented that represents the procession of the illuminated region while the measurement is being acquired. 


\subsection{Summary}

This study addresses the problem of minimizing the total scan time and number of useful measurements acquired during the scanning process by automatically adjusting the scanning process based on the quality of the measurements obtained. A low-density raster scan is initially performed to generate a quality-based region map that is then used as the basis for a series of high-density subscans. The multi-stage scanning approach is shown through experimentation to significantly reduce the scanning time and to improve scanning efficiency. Automated processes are developed for finding the locally-optimal scanner viewpoint, selecting regions that are most likely to yield useful, non-redundant measurements that are unlikely to be discarded, and adapting scan overlap and density to further improve scanning efficiency. These automated processes, as well as the wealth of information provided by a suite of quality metrics, make it possible for a less skilled operator to generate high-quality range models.

Chapter 2 presents a comprehensive survey of contemporary quality metrics and identifies areas in which improvements can be made. In Chapter 3, a series of new and improved quality metrics are presented, as well as a method for using these quality metrics to both find the locally optimal scanner viewpoint, and to isolate areas of the total field of view in which high-density scanning is most likely to yield useful measurements. In Chapter 4, additional new and improved quality metrics are presented, as well as a method for adapting the data acquisition process to improve scanning efficiency. Also introduced in Chapter 4 is a new quality-based approach to merging both spatial and intensity measurements. Chapter 5 provides a summary of the contributions and innovations resulting from this study, as well as suggestions for future areas of research. 


\section{Chapter 2}

\section{Survey of Quality Metrics}

Quality metrics, within the field of laser range imaging, are used to quantify by how much some aspect of a measurement deviates from a pre-defined standard. Measurement quality evaluations are becoming increasingly important in laser range imaging for range image registration, merging measurements, and planning the next best view. Spatial uncertainty and resolution are the primary metrics of image quality; however, spatial uncertainty is affected by a variety of environmental factors. A review of how contemporary researchers have attempted to quantify these environmental factors is presented, along with spatial uncertainty and resolution, resulting in a wide range of quality metrics.

\subsection{Introduction}

All range images begin with a series of range measurements, and the quality of the range image depends upon the quality of each of those measurements. The quality of a range measurement depends on measurement uncertainty and measurement resolution; however, spatial uncertainty is also strongly affected by environmental factors such as return signal intensity and relationship to a measurement's immediate neighbours. One 
or more of these factors can be expressed as a metric representing the deviation of some quality attribute associated with the measurement from a pre-defined standard. Spatial measurement quality represents the degree of confidence one can place in how accurately a measurement represents the position of a real surface in the environment. Laser range scanners can also provide intensity information that may be used in representing the surface so quality attributes relating to return signal intensity are useful. In this chapter, contemporary approaches to evaluating measurement quality attributes are reviewed, including measurement uncertainty, return signal intensity, range, sampling density, and relationship to neighbouring points. This review focuses particularly on measurement quality metrics that can be adapted for automated systems. Within this context, measurement quality metrics provide a way to direct and terminate automated scanning procedures.

Perceptual quality metrics can be either objectively or subjectively defined [53]; however, only objective quality metrics are useful for automating data acquisition. For this reason, only objective quality metrics are considered here. Objective quality metrics can be further classified as referenced or unreferenced [53]. Unreferenced quality metrics use no benchmark so automated processes can only evaluate the change in a quality attribute in response to some action. Referenced quality metrics can be evaluated in the same manner as unreferenced quality metrics, but the size of the deviation of an attribute from a reference can be used to evaluate whether the measurement should be either retained or ignored. For this reason, referenced quality metrics are preferred for automated systems in which thousands, or even millions of measurements may be obtained.

Referenced range measurement quality metrics quantify the relationship of a quality attribute to some previously established benchmark or reference. These metrics can then be used to either compare methods or systems, or they can be used in an iterative process to maximize some qualitative attribute of a range image [54]. Quality metrics appear most often in the guise of a weighting parameter when merging measurements or data sets. 
Two important components of a referenced quality metric are a clearly-defined quality benchmark against which to compare the current state of the range image, and a quality scale to indicate the degree to which the range measurement quality attribute deviates from the benchmark.

In this chapter, metrics for quantifying the quality of measurements are reviewed. For purposes of discussion, these metrics have been classified as measurement uncertaintybased, signal intensity-based, range-based, and neighbourhood-based. As will be demonstrated, considerable work remains to ensure that the quality of measurements and points used to construct virtual models is effectively and comprehensively defined.

\subsection{Spatial Resolution}

The spatial resolution of a laser range scanner measurement is dependant on the size of the laser spot that illuminates the surface at the point the measurement is obtained. For pulsed laser systems, the spatial resolution is also dependant on the pulse length of the system. The spatial resolution can be divided into range resolution and angular resolution. Angular resolution is the minimum angular distance between features such that they can be resolved as separate features. Range resolution is the minimum distance between angularly-resolved features such that they can be distinguished as separate features [49]. The angular resolution of a laser range scanner is defined by the Rayleigh criterion [48] and represents the size of the smallest feature that can be angularly resolved [49] [50].

The laser projects a spot into the surface being scanned, and the region in which the surface intersects the laser spot is referred to as the beam footprint. Features within the beam footprint contribute to the return signal intensity which is used to obtain the spatial measurement that approximates the position of a portion of the surface [51]. The area covered by the beam footprint is generally not measured by laser range scanners so it is approximated by a model of the area of the laser spot that illuminates the area. 
Ideally, the laser spot area should be the same as the beam footprint area; however, environmental factors such as spatial discontinuities [52] or dense fog [55], can result in the beam footprint deviating from that predicted by the laser spot model. Moreover, if the surface normal is assumed to be oriented along the line of sight in the laser spot model then surface angulation can result in a discrepancy between actual and predicted beam footprint areas. Quality metrics provide a way to predict by how much the beam footprint of a measurement might deviate from that predicted by the laser spot model.

The spatial resolution of a measurement can be represented by the instantaneous resolution that assumes the beam footprint is stationary at the time the measurement is acquired, or the effective resolution that takes into account the procession of the beam footprint over the surface during the acquisition process. When the term resolution is used in this thesis, unless otherwise stated, assume that it refers to the instantaneous resolution. The term beam footprint and laser spot are also used interchangeably in this thesis, although the terms are strictly equivalent only when the surface is continuous within the laser spot.

\subsection{Measurement Uncertainty-based Metrics}

Measurement uncertainty is the most common attribute used to assess measurement quality. Range measurement uncertainty is generally modelled as an independent zeromean Gaussian process added to the quantity returned by the range sensor; that is

$$
\hat{\mathbf{x}}=\mathbf{x}+\mathbf{e}
$$

where $\mathbf{x}$ is the ground truth position or surface characteristic, $\hat{\mathbf{x}}$ is the quantity returned by the sensor, and $\mathbf{e} \sim \aleph(0, \Sigma)$ is the additive zero-mean Gaussian noise process with measurement covariance $\Sigma$. This may not always be a valid assumption; environmental effects and non-linear bias in the sensors may cause the observed measurement distribu- 
tion to become distinctly non-Gaussian. In practice, Gaussian models provide the benefit of simplifying mathematical analyses and result in an approximation of how a system should behave under a broad range of circumstances. Non-Gaussian models are highly situation-dependant so are rarely used for predicting measurement uncertainty.

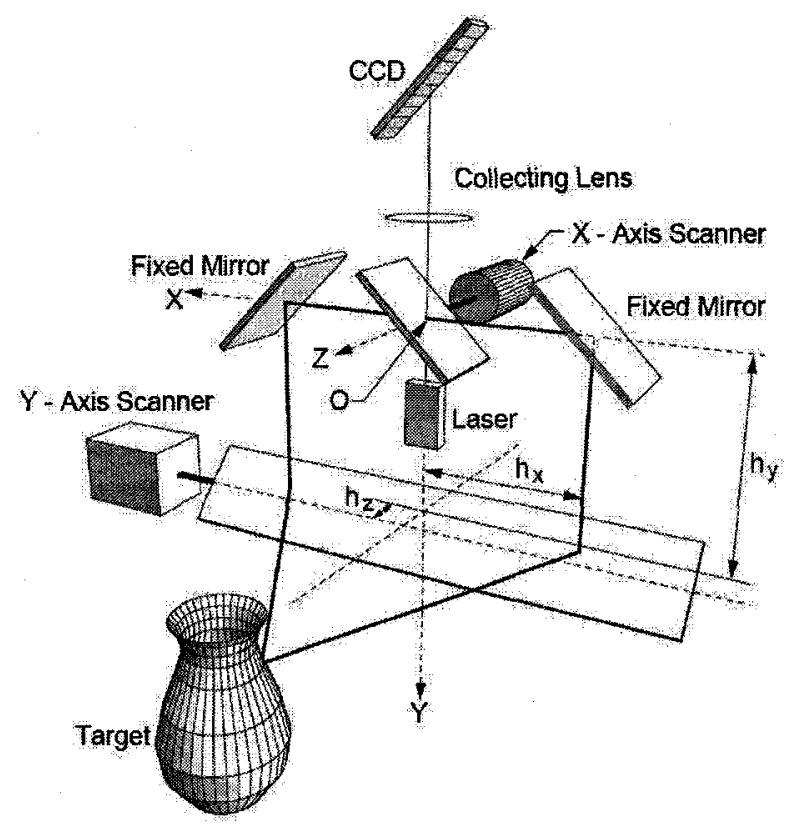

Figure 2.1: Example of a fixed-viewpoint laser range scanner employing dual-axis galvanometer-controlled rotating mirrors (Modified from Figure 6b of [56]).

The uncertainty associated with the range sensor is referred to here as the radial error and is one attribute that can be used to evaluate measurement quality. Rotational or translational position are referred to here generically as positional error and represent two more attributes that can be employed to evaluate the quality of a measurement. Figure 2.1 shows one example of a triangulation laser range scanner system in which the angular position of the laser spot on a surface in the environment is controlled by two rotating 
mirrors. Similar dual-axis optical scanning configurations are used in time-of-flight (TOF) systems and other laser range systems by combining orthogonal galvanometers, rotating mirrors, or motors. As a result, the geometrical model and measurement uncertainties can be generalized to a variety of laser range scanning systems.

\subsubsection{Measurement Uncertainty}

Measurement uncertainty is represented by a covariance matrix, generally based on a model of the root mean square (RMS) sensor error along each axis of motion employed by the scanner, and on a model of the error associated with the range sensor. Sensor variance is often based on a model of the sensor error, rather than on the spread of repeated measurements acquired in situ, because it is often not practical to obtain a large enough set of repeated measurements to derive a situation-specific variance profile. These models are generally obtained under ideal conditions for specific materials and surface orientations. As a result, there can be a significant discrepancy between the model sensor variance and what would be observed using a repeated measures approach in the field. For example, if the variance model of a system was based on white cardboard then the model variance would significantly underestimate the variance resulting from black felt [57]. This can be a significant issue where the type of material being scanned cannot be known a priori or where the object being scanned may consist of multiple types of material. In general, measurement uncertainty cannot be considered a sufficient quality metric on its own because it depends heavily on a variety of other attributes. In the following sections, various attributes that can result in true measurement uncertainty deviating from model-based measurement uncertainty are identified. 


\subsubsection{Positional Uncertainty}

Assuming a fixed-viewpoint scanner, such as the one shown in Figure 2.1, the positional uncertainty is a function of the mechanisms used to control the orientation of the laser and the photosensor [56]. These mechanisms are typically precision galvanometers or rotating motors, and the positional uncertainty reflects the variation in real laser/sensor orientations when the galvanometer or motor indicate that it has achieved a given angular position. In the case of fixed pattern projection systems, error positioning is often due to the stability of the opto-mechanical system. The acquisition of range and angular position measurements are generally synchronized, but synchronization errors, or jitter, can result in the true angular position differing from the angular position at the instant the range measurement is acquired [58].

Although the laser is often modelled as originating either from the scanner viewpoint or from a fixed point near the viewpoint, its true origin may vary depending upon the scanner geometry [59]. Well-calibrated laser range scanner systems account for this complexity; however, the transformation between sensor data and spherical or Cartesian coordinates can introduce errors [60] [61]. As a result, rotational uncertainty may not be constant as is often assumed. A similar situation arises for laser range scanner systems using motor-controlled rotating bases. Thermal effects, wobble and jitter, and mirror non-planarity can also cause the final reflection point position and output orientation to deviate from a Gaussian distribution.

\subsubsection{Radial Uncertainty}

Range measurement uncertainty depends on how the interaction of the laser with the surface is measured. In time-of-flight (TOF) systems, the range is determined by the time between the pulse being generated and being detected. In triangulation systems, the range measurement depends on the position of the signal peak on a photodetector 
array. In both cases, a significant portion of the range measurement uncertainty is the ambiguity of the location of the signal peak.

Range uncertainty is typically assumed constant for TOF scanners, as shown in Figure 2.2. Specifically,

$$
\sigma_{R}=\frac{c}{2} \sigma_{\tau}
$$

where $\sigma_{R}$ is the range measurement error, $c$ is the speed of light and $\sigma_{\tau}$ is the time measurement error. The last term represents the uncertainty in the temporal location of the signal peak. This is found by

$$
\sigma_{\tau}=\sqrt{\frac{T_{r}^{2}}{\mathrm{SNR}}}
$$

where $T_{r}$ is the pulse rise time and SNR is the signal-to-noise ratio [33] [62]. The range measurement error is determined by the signal bandwidth [62], amplitude of the return signal [63], thermal drift [63] [64], crosstalk between the transmitter and receiver [64], timing jitter [65], and non-uniformities and changes in the returning signal shape [62] [64] [65]. For example, different surface materials can change the shape of the return signal resulting in significantly different error distributions [57]. Moreover, feedback within the sensor can result in a measurement being affected by the previous measurement, violating the assumption that there is no correlation among range measurements.

Laser motion while the signal is being emitted is negligible for pulse TOF systems because the pulse duration is so short, but it can affect continuously modulated laser systems. This motion can distort the return signal and introduce ambiguity into the true measurement. Consider, as well, that the range measurement equation is given by

$$
R=\frac{c \tau}{2}
$$

where $\tau$ is the propagation delay [33] [47] [66]. This assumes that the time of flight between the laser and the surface is equal to the time of flight between the surface and 


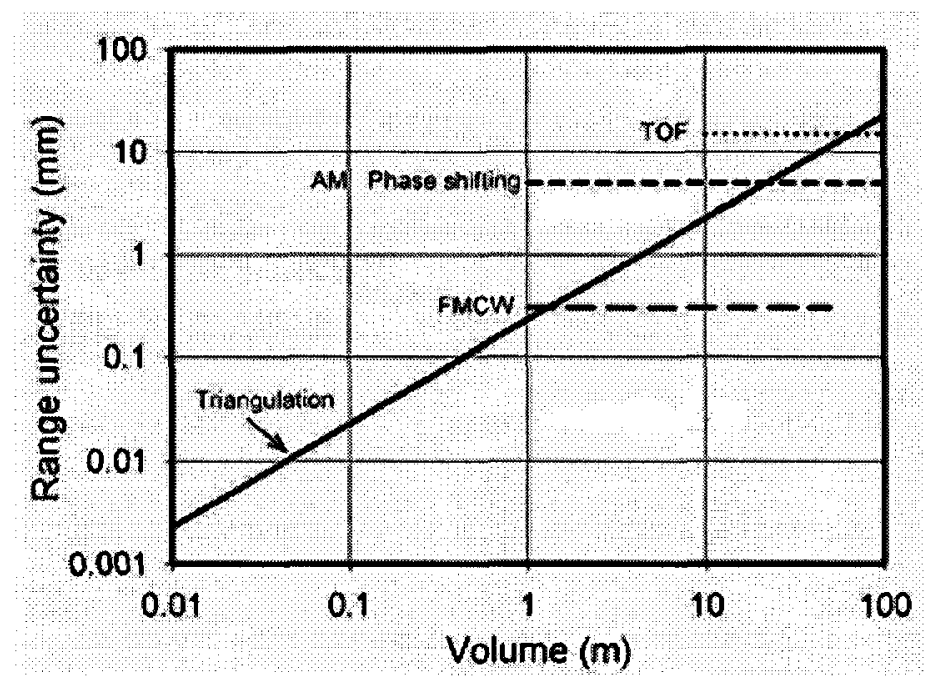

Figure 2.2: Common range uncertainties for Amplitude-Modulation Continuous Wave (AM), Frequency-Modulation Continuous Wave (FMCW), Time-of-Flight (TOF) and triangulation scanners up to $100-\mathrm{m}$ effective range (representing Volume in this graph). The range measurement uncertainties of all but the triangulation scanners are considered constant with respect to range (Modified from Figure $2 \mathrm{~b}$ of [33]). 
the sensor. This may not always be the case, especially if the true origin of the laser pulse varies as a function of the mirror angles.

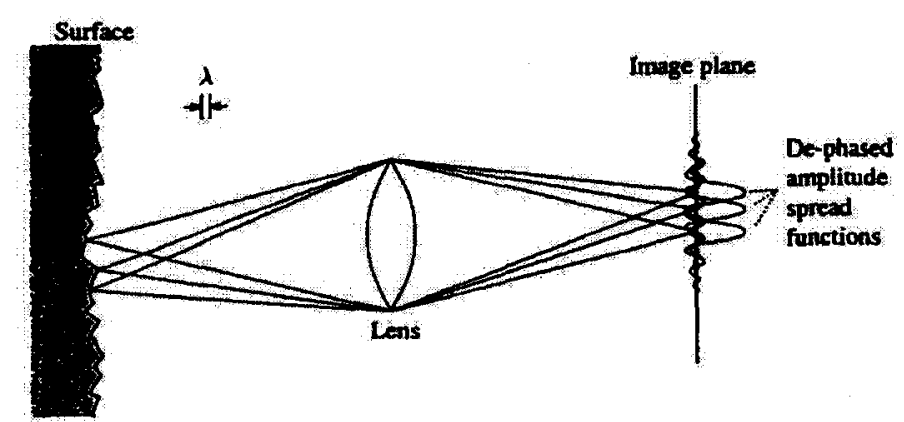

Figure 2.3: Speckle noise arises from the interference of a series of diffraction patterns, each generated by a speckle element (Modified from Figure 2.11 of [67]).

The peak uncertainty of a triangulation scanner is typically dominated by speckle noise [68]. This can be modeled as

$$
\sigma_{a}=\frac{\lambda f}{D \cos (\beta) \sqrt{2 \pi}}
$$

where $\lambda$ is the laser wavelength, $f$ is the focal length of the receiving lens, $D$ is the diameter of the collecting lens, and $\beta$ is the Scheimpflugg angle of the photodetector array [15]. Speckle noise arises when speckle elements on the surface illuminated by the laser spot are large when compared to the wavelength of the laser light [68] [69]. Under this assumption, each speckle element becomes a point emitter with respect to the photodetector array. Interference patterns are generated when each speckle element reflects light from the laser onto the photodetector array [67] [69], as shown in Figure 2.3. There, they constructively and destructively interact to form a speckle image on the photodetector array [67]-[69]. 


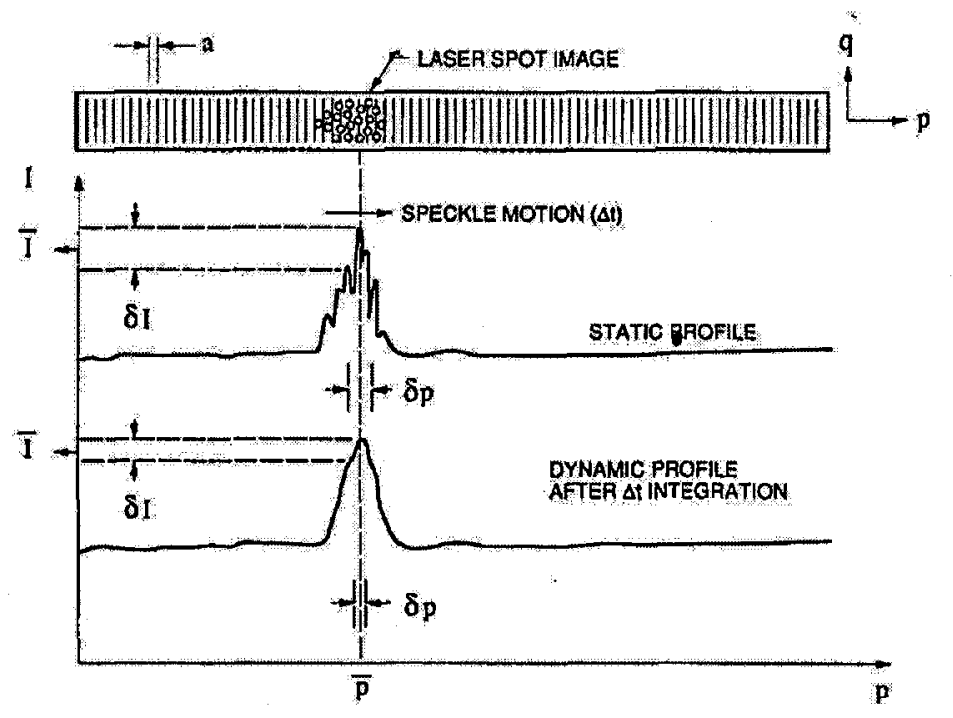

Figure 2.4: Speckle noise is reduced by integrating the measurement over several sampling intervals (Modified from Figure 3 of [70]).

Speckle noise is generally countered by integrating a single measurement over several intensity samples as the laser spot is moved over the surface being scanned [70]. Figure 2.4 shows a typical intensity profile without integration (static profile) and with integration (dynamic profile). This is complicated by the need to minimize aliasing by ensuring that the measurements are, where possible, separated by a distance less than the radius of the laser spot [52]. Similarly, the range uncertainty in an amplitude-modulation continuouswave (AM) scan can be decreased by increasing the sampling rate [71].

\subsubsection{Environmental Effects}

The mechanical effects described in the previous section can be included in a model of expected range and rotational uncertainty; however, many environmental factors, summarized in Table 2.1, can cause the true measurement uncertainty to deviate from 
Table 2.1: Environmental factors affecting measurement uncertainty.

\begin{tabular}{|c|c|}
\hline Error Source & Effect \\
\hline Range & Range uncertainty generally increases with range \\
\hline Angle of Incidence & Range uncertainty increases with increased angle of incidence \\
\hline Surface Material & $\begin{array}{l}\text { Translucent non-homogeneous materials increase range uncer- } \\
\text { tainty }\end{array}$ \\
\hline Surface Complexity & Surface discontinuities introduce range errors \\
\hline Reflectivity & Range uncertainty increases with a decrease in reflectivity \\
\hline Ambient Lighting & Range uncertainty increases with an increase in ambient lighting \\
\hline
\end{tabular}

the model. For example, measurement uncertainty can increase with increasing incidence angle [72]-[75], a reduction in surface reflectivity [57] [76], and an increase in ambient lighting [51] [77].

Equation (2.5) assumes that the size of the spot projected onto the photodetector array has not been distorted by occlusion, surface orientation or other environmental effects. Figure 2.5 shows the effect of laser spot distortion arising from a surface discontinuity [52]. In this case, the discontinuity occludes part of the laser spot so that the spot centroid no longer coincides with the signal peak. This introduces an error into the horizontal location of the signal peak, denoted here as $\Delta x$. This results in a range error $\Delta z$ which is compounded by the surface orientation with respect to the direction of the laser. The deviation of the surface normal from the laser path is denoted here as $\gamma$. Sudden changes in surface height are not uncommon and represent a reduction in measurement quality that is not captured by model-based measurement uncertainty.

Different surface materials can also affect the accuracy of range measurements. Figure 2.6 demonstrates the effect of a partially translucent material like marble in which the 


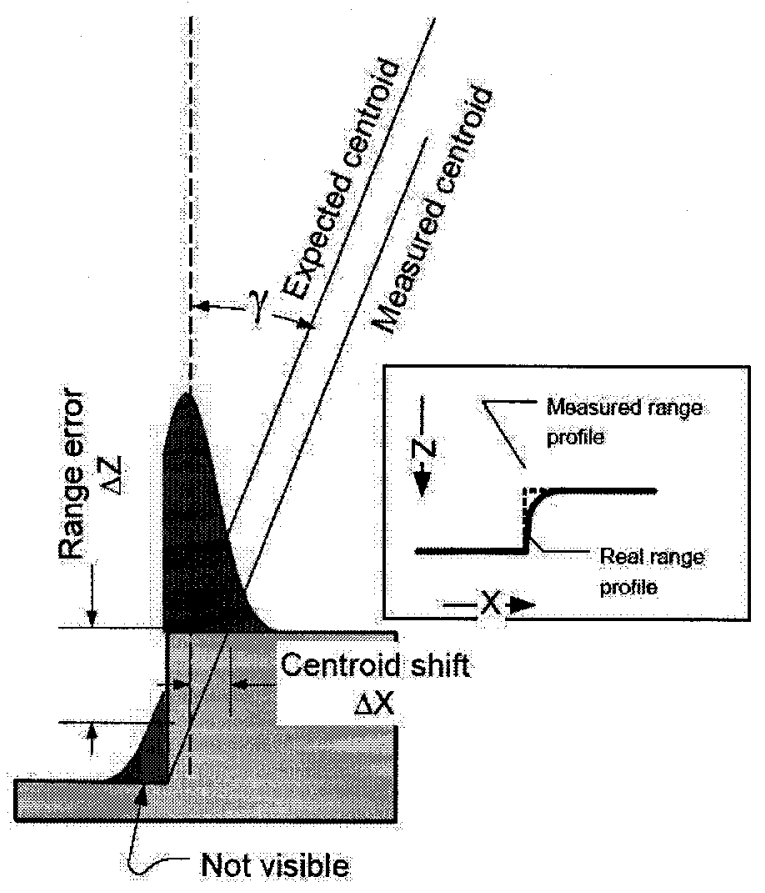

Figure 2.5: A range discontinuity results in a shift $(\Delta x)$ in the position of the centroid in a triangulation laser range scanner. This results in a range error $\Delta z$ (Modified from Figure $7(\mathrm{a})$ of [52]). 
laser may penetrate part-way into the surface before sufficient light is reflected to estimate the distance to the surface [52]. In this case, the range measurement does not represent the surface of the material, and the actual range measurement obtained depends on the reflective and refractive qualities of the material. According to Beraldin et al. [78], translucent surfaces like marble change the shape of the laser spot on the CCD array of a triangulation scanner, resulting in the range estimate being in error. As well, the nonhomogeneity of the material increases the range measurement uncertainty [33]. Translucent non-homogeneous materials can also feature a greater measurement uncertainty as well as a bias that increases with the distance between the scanner and the surface [79].

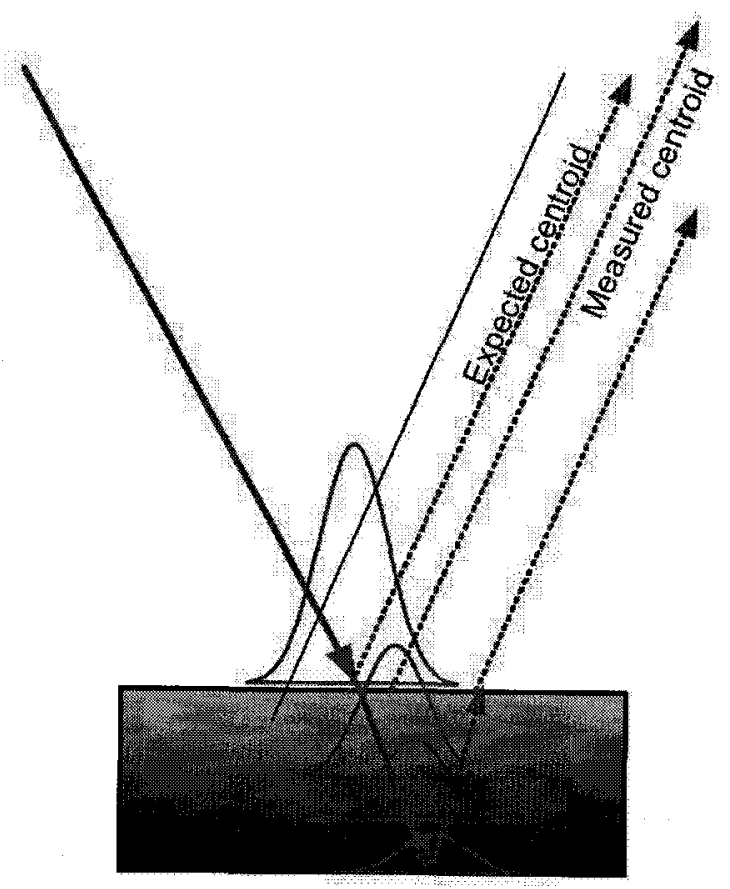

Figure 2.6: Range errors can result from the laser penetrating the surface of the material being scanned (Modified from Figure 6 of [52]). 
Surface complexity is not limited to variations in the height and frequency of surface structures; transitions between areas of different surface reflectivity can affect the accuracy of a range measurement [52] [80], as illustrated in Figure 2.7. Different materials with different reflectivity properties can also generate very different range measurement uncertainties [57]. The change in reflectivity for different portions of the laser spot results in a shift in the signal peak that introduces an error into both the range measurement and return signal intensity, a topic discussed in Section 2.4. Moreover, a reduction in surface reflectivity can result in an increase in range measurement uncertainty [77].
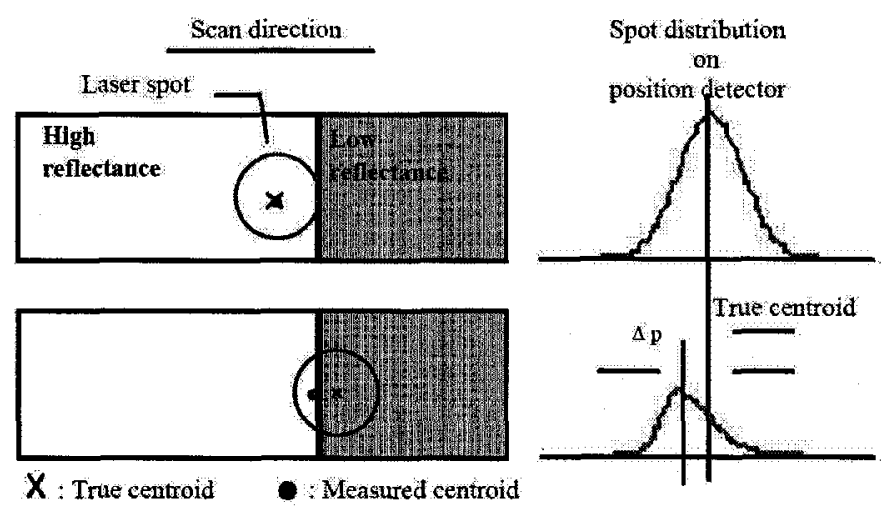

Figure 2.7: Transitions between regions of different surface reflectivity can affect the accuracy of the range measurement (Figure 1 of [80]).

Increasing the surface orientation with respect to the line of sight of the scanner can result in an elongation of the laser spot which increases peak detection uncertainty [75]. This problem is most pronounced when the length of the baseline is significant with respect to the distance to the surface, as is the case with triangulation laser range scanners, even when operating in the far field. Moreover, increased surface orientation with respect to the line of projection of the laser increases the spot size on the surface, resulting in more speckle elements contributing to the spot projected onto the CCD array. 
Because the range uncertainty of triangulation laser range scanners is dependant upon the surface orientation, model-based range uncertainty is not sufficient to represent the quality of a range measurement.

\subsubsection{Measurement Uncertainty as a Quality Metric}

Measurement spatial uncertainty has often been used as a way to quantify the quality of the measurement. For example, Sequeira et al. [2] [3] and Sequeira and Goncalves [4] used range sensor uncertainty as part of a reliability metric generated from the weighted sum of measurement attributes. They recognized that spatial uncertainty is not a sufficient metric so combined it with other measurement quality metrics. The combining of quality metrics to generate a more holistic view of measurement quality is discussed in Section 2.7. Some range sensors, such as the triangulation scanner shown in Figure 2.2, have range measurement variance that increases with the square of the distance between the scanner and the surface [15] [33] [41] [47] [81]. In this case, using range sensor uncertainty as a quality metric means that measurements closer to the scanner are considered to be of higher quality.

If the measurements are being merged using a modified Kalman minimum variance estimator (MKMV) approach [82] [83], then the measurement variance becomes a function of the number of measurements that are merged to form a point in a virtual model. Moreover, the merged measurements could be obtained from different viewpoints so range measurement uncertainty alone is insufficient as a quality metric. To counter this problem, the covariance matrix may be used as a multi-dimensional quality metric. For example, using the MKMV approach, two measurements $\hat{\mathbf{x}}_{i}$ and $\hat{\mathbf{x}}_{j}$ are merged to form a point $\mathbf{x}$ in the virtual model. The point is generated using the weighted sum

$$
\mathbf{x}=W_{i} \hat{\mathbf{x}}_{i}+W_{j} \hat{\mathbf{x}}_{j}
$$


where

$$
W_{i}=\Sigma_{j}\left(\Sigma_{i}+\Sigma_{j}\right)^{-1}
$$

and

$$
W_{j}=\Sigma_{i}\left(\Sigma_{i}+\Sigma_{j}\right)^{-1}
$$

are the weighting factors. As a result, the position of $\mathbf{x}$ is closest to the measurement with the smallest covariance. In effect, $W_{i}$, for example, becomes a quality metric for measurement $\hat{\mathbf{x}}_{i}$; the location of the point represents the integration of multiple measurements that maximizes the quality of the point from the perspective of measurement uncertainty.

One drawback of Sequeira's weighting method is that it was only applied to radial uncertainty. Table 2.1 illustrates the reasoning behind considering only radial uncertainty: it is the attribute that is generally affected by environmental factors. In Sequeira's case, the metric was only applied to range images and not to the merged data so this approach was sufficient for the purpose for which it was designed. Rotational uncertainty could be assumed constant and, thus, ignored. The method, however, is not generalizable to data merged using the MKMV method. Consider that the covariance of $\mathbf{x}$ is found by

$$
\Sigma^{-1}=\Sigma_{i}^{-1}+\Sigma_{j}^{-1}
$$

so the radial and rotational uncertainties of $\Sigma$ are less than the radial and rotational uncertainties of either $\Sigma_{i}$ or $\Sigma_{j}$. If only the radial uncertainty is considered then the reduction in rotational uncertainty is never taken into account. Similar issues arise when combining data from multiple types of scanners, each which may have different radial and rotational uncertainties.

The MKMV weighting factors, although effective quality metrics for measurement merger, are less effective for representing the quality of the measurement from the 
perspective of spatial measurement uncertainty. Ideally, an uncertainty metric should represent the uncertainty of a measurement as a scalar value so that the relative quality of measurements can be compared along a single vector rather than within a multidimensional space. On the other hand, reducing a multi-dimensional parameter to a single dimension risks losing potentially important information so the choice of unidimensional representation must be made carefully.

Although the covariance matrix approach addresses the issue of ignoring potentially valuable information in the position uncertainty attribute, it does not address the issues of surface complexity and orientation increasing the effective measurement uncertainty above the level predicted by the model. As a quality metric, range uncertainty and even measurement covariance are useful quality metrics but not sufficient by themselves. In particular, metrics evaluating surface spatial complexity, surface orientation, and changes in surface reflectivity need to be examined to augment measurement spatial uncertainty as a quality metric.

\subsection{Signal Intensity-based Metrics}

It was noted in Section 2.3.4 that a decrease in surface reflectivity can result in an increase in measurement uncertainty. Surface reflectivity can be assessed by examining how the intensity of the received signal varies from what would be expected for a surface of known reflectivity; however, signal intensity measurements can vary significantly as a result of such factors as range [76], high incidence angles [72] [75] [76], low reflectivity [57] [64], atmospheric attenuation [84], sharp discontinuities [33] [85], and translucency of the material being scanned [33] [41]. For example, the return signal intensity decreases with an increase in angle of incidence and decreases with an increase in distance between the scanner and the surface when the transmitted signal power remains constant [76]. As a 
result, quality metrics provide a way to predict the extent to which the actual reflectivity of a surface might deviate from that predicted from the return signal intensity.

Figure 2.5 illustrates that surface discontinuities can result in range errors; however, a change in the shape of the signal intensity profile in a triangulation laser range scanner can also result in a reduction in return signal intensity. When the shape of the peak is sufficiently distorted, as is the case with mixed measurements, it becomes difficult to locate its centroid. Laser spots that cross edges can result in smeared or multiple return signals that result in ambiguous range measurements, what is referred to as mixed measurement error [42] [76]. Mixed measurements are a result of receiving reflected energy from two surfaces within the laser spot and are often interpreted as a range measurement somewhere between the two surfaces [51] [77] [86]. Hebert and Krotkov [51] referred to the interdependence of measured range with signal intensity as range/intensity crosstalk. Time-of-flight systems calculate range by comparing the return signal to the transmitted signal so are more sensitive to signal intensity changes. Figure 2.8 shows that a discontinuity in surface reflectivity can also reduce the return signal intensity [52]. As a result, quality metrics provide a way to predict the extent to which the spatial position of the measurement might be in error as a result of the return signal intensity deviating from that predicted using a model of the laser range scanner optics.

Some surfaces may be difficult, if not impossible, to scan because the return signal is diffusely scattered, what is referred to as volumetric scattering [22] [42]. Surfaces that exhibit this property include glass, hair [42] and grass [22]. Figure 2.6 illustrates that translucent materials can also reduce the strength of the return signal [52]. Other surfaces are excessively absorbent so insufficient signal is returned to obtain a range measurement, while other surfaces may be so highly reflective that the photodetector is saturated [42]. The absence of a return signal, what can be referred to as a non-return measurement, can be a valuable piece of information but is almost always discarded. 


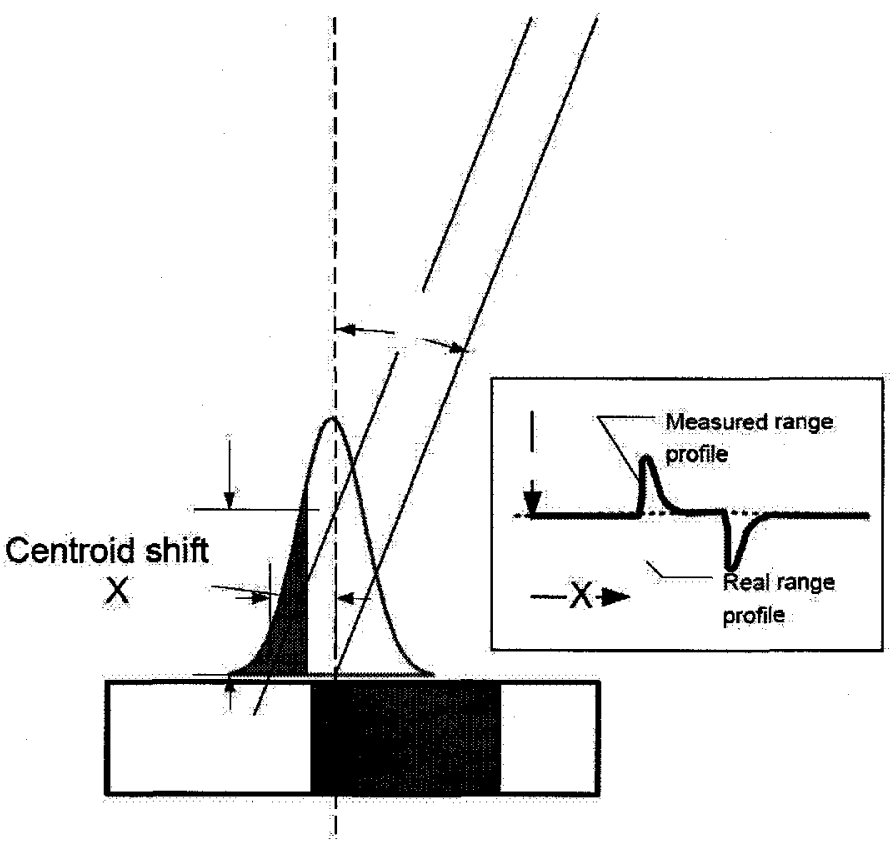

Figure 2.8: A discontinuity in surface reflectivity results in a shift $(\Delta x)$ in the position of the centroid in a triangulation laser range scanner. This results in a change in return signal intensity (Modified from Figure 7(b) of [52]). 
Table 2.2: Environmental factors affecting return signal intensity.

\begin{tabular}{|c|c|}
\hline Error Source & Effect \\
\hline Range & Return signal intensity decreases with an increase in range \\
\hline Angle of Incidence & $\begin{array}{l}\text { Return signal intensity decreases with increased angle of } \\
\text { incidence }\end{array}$ \\
\hline Surface Material & $\begin{array}{l}\text { Translucent non-homogeneous materials can reduce return } \\
\text { signal intensity }\end{array}$ \\
\hline Surface Complexity & Surface discontinuities can reduce return signal intensity \\
\hline Reflectivity Changes & $\begin{array}{l}\text { Return signal intensity decreases with a decrease in reflectiv- } \\
\text { ity }\end{array}$ \\
\hline
\end{tabular}

Given a reference material, the change in return signal intensity can be modelled as a function of range. A shift in the return signal intensity from the model value can then be used as a metric of the quality of a measurement. Measurement spatial uncertainty is also affected by return signal intensity so both variables are important in assessing measurement quality, and neither are sufficient by themselves. Moreover, signal intensity shifts can indicate the presence of mixed pixels and surface material transitions, either of which may introduce errors into the range measurement. The challenge is in how to determine the cause of the intensity shift, given that only the spatial position and deviation in signal intensity from a model value are known. Deviations from model return intensity can arise from several different environmental conditions so return intensity, even when combined with spatial position and model spatial uncertainty, is not sufficient to completely represent the quality of a measurement. Table 2.2 summarizes the factors that affect return signal intensity. 


\subsubsection{Intensity as a Quality Attribute}

Signal intensity is rarely used as a quality metric; it is more often used as a weighting factor for combining measurements. For example, Godin et al. [87] used the compatibility of signal intensities between correspondence pairs of measurements prior to Iterative Closest Point (ICP) registration. Given two intensity measurements $h_{i}$ and $h_{j}$, the compatibility $C\left(h_{i}, h_{j}\right)$ is found by

$$
C\left(h_{i}, h_{j}\right)=e^{-\frac{\left(h_{i}-h_{j}\right)^{2}}{\sigma_{c}^{2}}}
$$

where $\sigma_{c}^{2}$ is an estimate of the reliability of the intensity measurements. In this equation, $h_{i}$ and $h_{j}$ are quality attributes associated with measurements $\hat{\mathbf{x}}_{i}$ and $\hat{\mathbf{x}}_{j}$ respectively; however, this metric only assessed the quality of association between two measurements, not the quality of each measurement. Fiocco et al. [88] defined a reflectivity quality metric for each measurement. It took the form

$$
\rho=\left\{\begin{array}{cc}
1 & \rho_{\min } \leq \rho_{i} \leq \rho_{\max } \\
0 & \text { otherwise }
\end{array}\right.
$$

where $\rho_{\min }$ and $\rho_{\max }$ defined the minimum and maximum acceptable reflectivity of the surface, and $\rho_{i}$ was the observed surface reflectivity. Sequeira et al. [2] [3] simply applied a weighting factor to the detected signal intensity.

One drawback of Fiocco's method is that it employs a binary scale which, while useful for the application for which is was designed, lacks the generalizability of a sliding scale. Sequeira's approach of using a weighting factor avoids this problem, but does not address the issue of the ideal reflectivity changing with an increase in range. As with Fiocco's method, the weighted intensity approach used by Sequeira was sufficient for the application for which it was designed but is not applicable to medium-range scanning without some modifications to take into account the relationship between range and return signal intensity. Fiocco avoids this problem by using reflectivity which is rangeindependent. 


\subsection{Range-based Metrics}

It is noted in Section 2.3.3 that measurement spatial uncertainty generally increases with increased range and, in Section 2.4, that return signal intensity generally decreases with increased range. The range measurement itself can be used to represent the quality of a measurement. For example, Sequeira et al. [2] [3] and Fiocco et al. [88] each used the range portion of the measurement as part of their reliability metrics. Figure 1.4 graphically demonstrates how the quality of a measurement decreases as the distance between the scanner and the surface that generated the measurement increases.

In general, the farther a surface is from the scanner, the larger the area encompassed within the laser spot. The size of the spot projected onto a surface is represented by the beam width at the point of intersection. The beam width depends on the distribution of irradiance which is often assumed to follow a Gaussian distribution. Specifically,

$$
I(r, \zeta)=I_{c} e^{-\frac{2 r^{2}}{w(\zeta)^{2}}}
$$

where $I_{c}$ is the irradiance of the beam along the central axis, $r$ is the radial distance perpendicular to the central axis, and $w(\zeta)$ is the spot radius a distance $\zeta$ from the beam waist [89] [90]. Figure 2.9 shows the irradiance profile centered on the central axis, and the spot size $w(\zeta)$ as a function of distance from the beam waist.

The surface formed by $w(\zeta)$ represents the distance $r$ from the central axis at which the beam irradiance falls to $1 / e^{2}=0.135$. As a result, the volume bounded by $w(\zeta)$ represents the region within which $86.5 \%$ of the beam irradiance is contained [89]-[91]. The laser spot defined in this way represents the portion of the surface being scanned from which most of the laser irradiance is being reflected. As a result, the laser spot represents the smallest region that can be resolved by the laser range scanner.

The boundary of $w(\zeta)$ can be approximated by:

$$
w(\zeta)=w(0) \sqrt{1+\left(\frac{\zeta}{\zeta_{0}}\right)^{2}}
$$




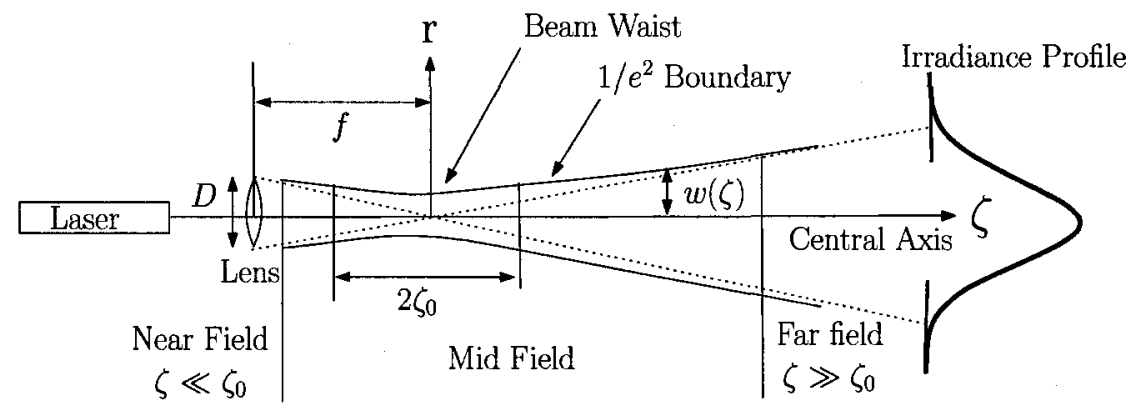

Figure 2.9: Laser beam $1 / e^{2}$ boundary. The near field is the region in which $\zeta \ll \zeta_{0}$ while the far field is the region in which $\zeta \gg \zeta_{0}$.

where $w(\zeta)$ is the beam radius, $w(0)$ is the radius of the beam waist, and $\zeta / \zeta_{0}$ is the ratio of the beam waist to the depth of focus of the beam $\zeta_{0}$. The depth of focus, illustrated in Figure 2.9, is defined by

$$
\zeta_{0}=\frac{\pi w^{2}(0)}{\lambda}
$$

where $\lambda$ is the laser wavelength. Meanwhile, the beam waist for an aberration-free optical system using a circular lens can be approximated by the Rayleigh diffraction equation

$$
w(0) \approx \frac{1.22 \lambda f}{D}
$$

where $D$ is the lens diameter and $f$ is the focal length of the lens [90]. The focal length also represents the distance from the lens to the beam waist.

Range can act as a proxy for the resolution of a measurement under the assumption that the focal length remains fixed and the surface is farther from the scanner than the beam waist. Under these conditions, measurements closer to the scanner can be considered to be of higher quality than those farther from the scanner. Although range is generally not referred to as an indicator of the quality of a measurement, this relationship is implied when the more distant of a pair of measurements is dropped as part of the registration process. 
Fiocco et al. [88] defined a distance quality metric based on the minimum and maximum range limits. In practice, a scanner is bounded by the minimum and maximum effective range, defined by a variety of factors including the laser power, beam spread, and photodetector sensitivity. Sequeira et al. [2] [3] simply applied a weighting factor to the range measurement to obtain a quality metric.

Only long range scans (those for which $\zeta \gg \zeta_{0}$, referred to as far field measurements [89]) are guaranteed to have measurement resolution decrease with range. Medium range scanners may be used for surfaces that are at, or even less than, the distance to the beam waist. Surfaces that are closer than the beam waist have a direct relationship between resolution and range, as shown in Figure 2.9. In this case, measurement quality decreases with distance. As a result, Fiocco's and Sequeira's methods are only applicable to the situation for which they were designed; laser range scanners in which the surface is farther from the scanner than the beam waist. For medium-range scanning, the surface may be placed such that it coincides as much as possible with the beam waist. A more general-purpose resolution-based quality metric should be applicable to both long and medium-range scanner data, as well as data from scanners with multiple focal lengths. The use of laser spot size in assessing measurement quality is addressed in Section 2.6.

\subsection{Neighbourhood-based Metrics}

Attributes such as surface orientation, or spatial or reflectivity discontinuities, cannot be determined from single measurements; they can only be inferred from groups of measurements located in close spatial proximity to each other. Spatially-related measurements are referred to here as a neighbourhood and are used to model a small portion of the surface being scanned to predict some aspect of that surface, such as its orientation. The class of neighbourhood-based metrics encompasses all quality metrics defined by the neighbourhood of a measurement. 
Neighbourhood-based quality metrics attempt to infer some aspect of a measurement by its relationship to its immediate neighbours. For purposes of discussion, a neighbourhood is defined as a point $\hat{\mathbf{p}}$ and the set of all points $P=\left\{\hat{\mathbf{p}}_{0}, \ldots \hat{\mathbf{p}}_{K}\right\}$ considered to be the immediate neighbours of $\hat{\mathbf{p}}$ by some commonly-accepted criteria. It is assumed that this criterion is either the Euclidean or the rotational distance, although the discussion could apply to other distance metrics.

Two neighbourhood-based quality metrics are considered: those based on inter-point distance, and those based on vertex orientation with respect to the line-of-sight. The former is a measure of the density of the measurements in a neighbourhood which, in turn, indicates how finely the surface has been sampled. The latter is used to estimate the orientation of the surface at a spatial location of the measurement, and is the most commonly used quality metric after measurement uncertainty. Surface complexity can also be evaluated using edge detection techniques. For surveys on edge detection techniques, see Argyle [92], Davis [93], Peli and Malah [94], Ziou and Tabbone [95], Trichili et al. [96], Xiao et al. [97], and Basu [98].

\subsubsection{Distance Metrics}

Distance metrics are typically used to evaluate two attributes: distance to neighbouring points, and the density of points in the neighbourhoods. The latter is referred to as sampling density which is the number of measurements per unit area of the surface being modelled. Densely sampled surfaces have the greatest possibility of detecting important surface features that might be missed by more sparse sampling methods. On the other hand, dense scanning techniques generate a large number of points, many of which may be redundant if the surface being scanned lacks significant surface features. With respect to quality, densely sampled surfaces, to within certain limits, have the greatest probability of 
generating high-quality models so sampling density is a measure of the potential quality of the final model.

According to Shannon sampling theory, given a bandlimited signal, the sampled signal will contain all the information in the bandlimited signal only if the sampling frequency is more than twice the signal bandwidth [99]. This is also known as the Shannon-Nyquist sampling theorem [100] or simply the Nyquist sampling theorem [101]. This means that the distance between samples must be less than half the smallest feature size to be resolved [102]; that is,

$$
d<\frac{\Delta x}{2}
$$

where $d$ is the distance between samples, and the smallest feature size to be resolved, also referred to as the target resolution, is given by $\Delta x$. The signal bandwidth is referred to as the Nyquist frequency, and the Nyquist rate, equal to twice the Nyquist frequency, defines the frequency that must be exceeded by the sampling frequency. If the sampling frequency is less than or equal to the Nyquist rate then aliasing, or aliasing distortion, occurs [101]. On the other hand, measurement quality does not improve in proportion to the amount by which the sampling rate exceeds the Nyquist rate [103] so the sampling rate is often defined to be only slightly higher than the Nyquist rate. The Nyquist rate, therefore, represents a quality breakpoint.

Shannon sampling requires a bandlimited signal, and diffraction in the optical system ensures this by imposing a limit on the size of features that can be resolved. The Rayleigh criteria represents the resolution limit of the scanning system even if measurement noise were negligible [48] [49]. In the case of a perfectly focused, diffraction-limited optical system, laser physics still imposes a limit on the size of the feature that can be resolved, given by the Rayleigh criteria. If $\Delta d$ represents the minimum distance between beam footprint peaks at which they can be separately resolved then the Nyquist rate is given 
by

$$
f_{R}=\frac{1}{\Delta d}
$$

and the Nyquist frequency becomes $f_{N}=f_{R} / 2$. The smallest feature that can be resolved is given by the beam footprint width $2 \Delta d$.

If $2 \Delta d$ is large with respect to $d$, the inter-sample distance, then fine details are blurred [49]; however, if $d$ is too large then fine details are missed. It is convenient, in the absence of other information about the system, to choose a sampling density slightly less than the smallest angular beamwidth such that $d<\min \{w(\zeta)\}$ within the volume of interest. The goal of scanning a surface is to achieve an inter-sample surface distance less than or equal to the target resolution $\Delta x$, given that the laser scanner is, under ideal conditions, unable to resolve features at less than $2 \Delta d$. Sampling density and inter-sample distance, therefore, are useful in assessing model quality.

Klein and Sequeira [43] and Klein and Zachmann [104] compared the actual sample density $\beta(\mathbf{p})$ to the expected sampling density $F(\mathbf{x}, V, m)$. The expected sampling density was found using

$$
F(\mathbf{x}, V, m)=m \frac{\vec{n}^{T}(\mathbf{x}-V)}{R^{3} A_{\text {patch }}}
$$

where $V$ is the position of the scanner in the world, $m$ is the resolution, $A_{p a t c h}$ is the solid angle of the patch covered by a single "pixel", $\vec{n}$ is the unit normal of the surface at $\mathbf{x}$, and $\mathbf{x}$ is a point in the global coordinate system. If $\mathbf{x}$ is part of an unscanned surface, then $F(\mathbf{x}, V, m)=0$. These quality metrics were then used to perform a cost-benefit analysis of potential viewpoints. Specifically, they calculated the resolution quality of point $\mathbf{x}$ in the surface as seen from viewpoint $V$ using

$$
B(\mathbf{x}, V, m)=\min \left\{\beta_{\max }(\mathbf{x}), F(\mathbf{x}, V, m)\right\}-\min \left\{\beta_{\max }(\mathbf{x}), \beta(\mathbf{x})\right\}
$$

where $\beta_{\max }(\mathbf{x})$ is the maximum sampling density of $\mathbf{x}$, and $\beta(\mathbf{x})$ is the observed sampling density of $\mathbf{x}$. In this case, the benchmark is $\beta_{\max }(\mathbf{x})$. The benefit of this approach is that 
it combined measurement resolution, surface orientation, and sampling density into a quality metric for each point on a surface.

Fiocco et al. [88] used a less complicated method for defining the density of a set of measurements than proposed by Klein. They defined the density quality metric as

$$
s=\left\{\begin{array}{cl}
\frac{s_{\max }-s_{i}}{s_{\max }} & s_{i} \leq s_{\max } \\
0 & \text { otherwise }
\end{array}\right.
$$

where $s_{i}$ is the distance to the closest neighbour and $s_{\max }$ is the maximum acceptable distance. Meanwhile, Sequeira et al. [2] [3] used the weighted average distance between neighbouring points as a quality metric.

One drawback of the quality metrics employed by Klein, Fiocco, and Sequeira is that they ignore measurement spatial uncertainty which also affects the resolution of the system [49] [105]. In particular, spatial uncertainty makes it difficult to know precisely the extent of the region covered by each laser spot. Another drawback of these metrics is that they do not make clear whether quality is being assessed relative to the target resolution $\Delta x$ or the attainable resolution $2 \Delta d$. The former is generally constant while the latter depends on surface orientation, the presence of spatial or reflectivity discontinuities, and the size of the laser spot illuminating the surface. In some cases, $\Delta x$ may not even be attainable for certain combinations of range and surface orientation.

\subsubsection{Orientation Metrics}

A typical approach to generating the orientation of a measurement is to obtain a mesh model of the surface and use the normals of each of the mesh elements to estimate the normal of the surface at the measurement [67] [82] [106]-[108]. Orientation is often represented by the surface normal which is generally found by taking the average of the normals of all Delaunay facets that have this measurement as a vertex [82] [107]. The exception is Hoppe et al. [109] who preferred to use the normal of a plane fit to the 
neighbourhood of the measurement. The benchmark for the grazing angle attribute is the angle that generates the most accurate range measurement; that is, when the surface normal is oriented along the line between the surface and the scanner. Assuming the maximum grazing angle is one in which the surface normal is perpendicular to the line between the surface and the scanner, the scale of the grazing angle attribute is from 0 (best quality) to $\pi / 2$ (worst quality) radians. This is often represented as the cosine of the grazing angle [74] [107] which has a range of 1 (best quality) to 0 (worst quality). Whether the surface normal is calculated using mesh elements or planar fit, care must be taken when interpreting the result because the uncertainty in the surface normal is a function of the uncertainties associated with all measurements used to obtain the surface normal.

Often the deviation of the return signal intensity from the ideal Lambertian model is represented by the surface normal [67] [82] [106] [107] or grazing angle [110]. The reasoning is that the signal intensity decreases with increasing surface orientation, so surface orientation can be used as a proxy for signal intensity; however, return signal intensity is affected by all the factors summarized in Table 2.2 so this assumption is true only in the absence of other factors such as surface spatial complexity and changes in surface reflectivity. Surface orientation also affects the uncertainty of range measurements [111], particularly for triangulation laser range scanners, so surface orientation as a metric can affect quality metrics for both spatial uncertainty and return signal intensity.

Fiocco et al. [88] used the deviation of the line-of-sight to the scanner from the surface normal as a quality metric. This metric took the form

$$
\varphi=1-\frac{\varphi_{i}}{90}
$$

where $\varphi_{i}$ is the surface orientation deviation in units of degrees. Turk and Levoy [107] used the cosine of the grazing angle to weight measurements prior to Iterative Closest Point (ICP) registration. Soucy and Laurendeau [74] showed that the squared cosine of 
the grazing angle corresponds to the relative illuminance received by the photodetector. They used this metric to perform a weighted merge of measurements from different viewpoints such that

$$
\mathbf{x}=\sum_{i=1}^{N} W_{i} \hat{\mathbf{x}}_{i}
$$

where

$$
W_{i}=\frac{\cos ^{2}\left(\gamma_{i}\right)}{\sum_{j=1}^{N} \cos ^{2}\left(\gamma_{j}\right)}
$$

is the weighting factor associated with measurement $\hat{\mathbf{x}}_{i}$. The cosine of the grazing angle can be found using

$$
\cos \left(\gamma_{i}\right)=\frac{\vec{n}_{i}^{T} \hat{\mathbf{x}}_{i}}{\hat{R}_{i}}
$$

where $\hat{\mathbf{x}}_{i}$ is a measurement located $\hat{R}_{i}$ units from the viewpoint, and $\vec{n}_{i}$ is the unit normal to the surface at $\hat{\mathbf{x}}_{i}$. In this case, the coordinate system is assumed centred on the scanner viewpoint. Curless [67] employed a similar approach to merging measurements that cooccupied the same voxel. Soucy and Laurendeau [74] demonstrated that the reflectivity of the surface was directly proportional to the square of (2.24). Because measurement quality was expected to be directly proportional to the amount of light returned to the sensor, $\cos ^{2}(\gamma)$ would better represent measurement quality than $\cos (\gamma)$; however, this was based on the assumption that the reflectivity change was primarily caused by high surface orientation. The relationship is less clear when the surface reflectivity is more complex.

Scott et al. [110] suggested that basing quality solely on the grazing angle of a measurement ignores the objective effects of high grazing angle in favour of a more subjective metric. Surface orientation, in particular, ignores factors that affect the shape and peak height of the intensity profile such as surface reflectivity changes. Moreover, 
the surface normal is the average of the orientations along each Delaunay edge extending from a point. As a result, it is possible to have a wide range of vertex normals but a surface normal oriented along the line-of-sight. Finally, for systems in which the baseline is large with respect to range, the line-of-sight could be defined with respect to the photodetector, the laser, or the scanner origin, each yielding a different result. As a result, surface orientation is important but insufficient as a quality metric.

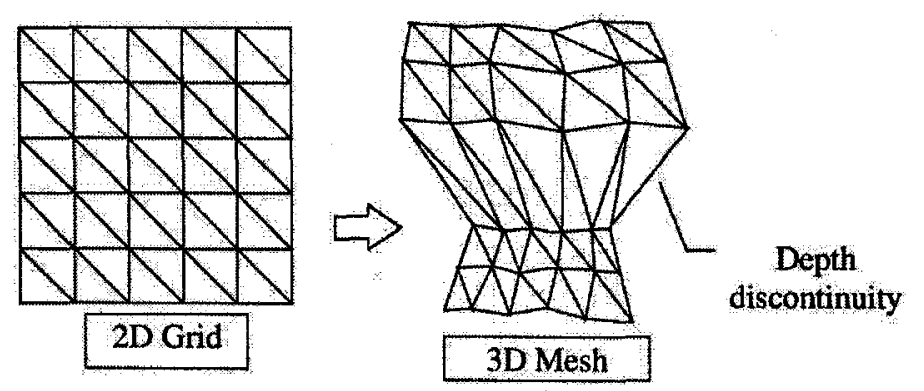

Figure 2.10: Range discontinuities result in elongation of Delaunay facets when viewed in three dimensions (Modified from Figure 4 of [3]).

An alternative to grazing angle for representing surface orientation of a range image obtained using a raster scan pattern is the facet edge length ratio. In this case, the ratio of longest to shortest edge of a Delaunay facet is used to assess the quality of the facet, and, by extension, its measurements. Sequeira used this approach to discard the facet if the ratio was too large [2]. Consider the image on the left in Figure 2.10 which represents a two-dimensional Delaunay triangulation of a range image; when seen in three dimensions, facets on a discontinuity are elongated with respect to their neighbours. The ratio between the longest and shortest edge should ideally be $1: 1$; that is, the triangles should be equilateral. As the surface orientation increases with respect to the line of sight from the scanner, the ratio between the longest and shortest edges increases. Specifically, 
given a facet $F_{i}$ with edges $E_{i}=\left\{e_{i, 1}, e_{i, 2}, e_{i, 3}\right\}$, the facet edge ratio $w_{i}$ can be found by

$$
w_{i}=\frac{\min E_{i}}{\max E_{i}} \in(0,1]
$$

The weighting factor $w_{i}$ decreases toward zero as the disparity between the longest and shortest edges increases.

(a)

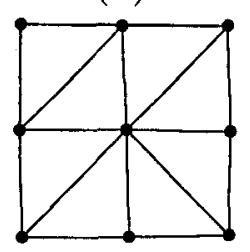

(c)

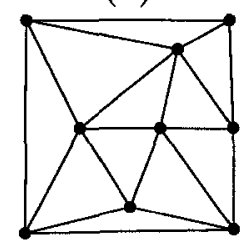

(e)

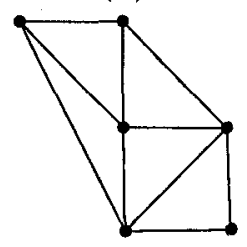

(b)

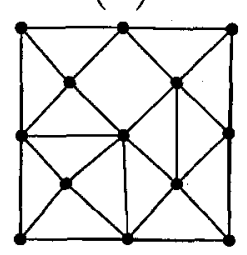

(d)

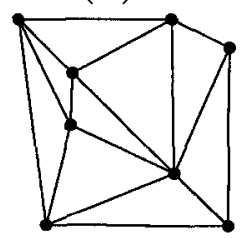

(f)

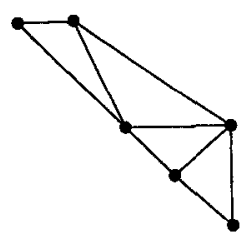

Figure 2.11: Facet ratio is most effective for regularly-spaced data, such as (a) and (b). As measurement distribution becomes less regular, facets with large facet ratios can emerge regardless of their relative range measurements.

The facet ratio represents a quality metric in which the neighbourhood is limited to the three measurements bounding the Delaunay facet. High quality measurements would be those in which the facet ratio was close to 1 , while those in which $w_{i}$ was very small would be considered to be low-quality measurements. Low-quality measurements 
would have elongated facets indicating steep surface slopes. A drawback of this method is that it is specifically designed to assess the quality of facets and can only be applied to measurements as a side benefit. Moreover, it is specifically designed to work with regularly-spaced raster patterns. Non-raster patterns can feature large edge ratios even if the surface is relatively flat, as illustrated in Figure 2.11. Arrangements (d), (e) and (f) contain facets with large facet ratios regardless of the range value associated with them. Although well-suited to the purpose for which it was designed, facet ratio is not easily adapted to use as a general-purpose quality metric representing surface orientation. Fiocco's method, as well as the more popular grazing angle metric described by (2.24), are better suited as general-purpose surface orientation quality metrics.

\subsection{Total Quality Metric}

Quality metrics are generally combined to generate an overall measure of quality, referred to here as a total quality metric. Scott et al. [54] cited two common examples of how quality metrics could be combined: weighted summation and composite binary pass/fail. The weighted summation approach takes the form

$$
C_{i}^{\text {total }}=\sum_{j=1}^{N_{C}} w_{j} C_{i, j}
$$

where $C_{i, j} \in[0,1]$ represents the $j^{\text {th }}$ quality metric. An example of this approach is the weighted average model used by Sequeira et al. [2] to determine the total quality of each measurement in a range image. To ensure that $C_{i}^{\text {total }} \in[0,1]$, the weight values can be restricted such that $\sum_{j=1}^{N_{C}} w_{j}=1$. Meanwhile, the binary product approach has the form

$$
C_{i}^{\text {total }}=\prod_{j=1}^{N_{C}}\left(C_{i, j} \geq C_{T, j}\right)
$$


where $C_{T, j}$ is a threshold quality limit for the $j^{\text {th }}$ quality metric. In this case, $\left(C_{i, j} \geq\right.$ $\left.C_{T, j}\right)=1$ when the quality metric equals or exceeds the threshold value, and $\left(C_{i, j}<\right.$ $\left.C_{T, j}\right)=0$ otherwise.

The choice of how quality metrics are combined depends on the application and the relative weight placed on each of the quality metrics. The weighted summation approach allows the researcher to tailor the contribution of each of the quality metrics to the overall measurement quality without any one metric dominating the result. For example, Fiocco et al. [88] experimentally derived the weights for each sensor used in the experiment. They also standardized the weighting factors such that each sensor technology could be represented by a single weighting factor that modified each of the metric weights. Sequeira et al. [2] [3] also used the weighted sum approach but did not indicate how the weights were derived. The binary product approach is effective if the goal is to simply exceed some preset quality level.

\subsection{Unresolved Quality Issues}

Several quality attributes are notably absent from contemporary, and even emerging, quality metrics. In particular, no quality metric has been developed to address the motion of the laser spot during the acquisition process. This is of particular interest in triangulation scanners where multiple sample intervals may be integrated to combat speckle noise. No quality metric has been defined to quantify the effect of measurement resolution. Even using range as a quality metric only addresses measurement resolution by proxy. In fact, neighbourhood-based metrics do not consider the issue of measurement density or proximity that is less than the measurement resolution of the system. No metric has addressed the problem of measurement repeatability, most likely because it requires multiple range images of the same surface, which substantially increases scanning time. Finally, surface complexity is only imperfectly evaluated using surface orientation. 
Measurement quality metrics are rarely combined into a total quality metric. As a result, operations such as measurement merge, range image registration, and deciding whether or not to delete a measurement are often based on inadequate information. For example, although a maximum likelihood merge of two measurements is statistically valid, the covariance matrix only partially describes the quality of the measurement. In fact, a measurement with relatively large covariance may be of substantially higher quality than a measurement with relatively small covariance when other factors such as distortion of

the signal peak and surface orientation are taken into account. A more comprehensive approach to applying measurement quality to manipulating measurements is required.

Finally, non-return measurements are generally treated as having no qualitative value so are often ignored during data collection. This means that information about regions of the environment that cannot be scanned is lost. Additional research is required to examine what can be learned about the environment being scanned from the absence of a return signal.

\subsection{Summary}

Quality metrics have featured significantly in contemporary research; however, most quality metrics have been designed for specific applications or specific algorithms, and are often used independently. Measurement uncertainty has been used extensively to represent measurement quality but many environmental factors affect measurement uncertainty, making it insufficient as an independent quality metric.

The relationship of range and resolution to measurement quality depends on the beam width. Additional work is required to better define the relationship between measurement quality and resolution for mid-field measurements where parallax must be taken into account. Sampling density has also featured in various forms as a quality metric, although most approaches are highly application specific. Absent from the literature is a more 
detailed analysis of how sampling density is related to measurement quality and how to quantify sampling density as a quality metric in a generalized fashion. Surface orientation has also been used extensively as a quality metric, although it is also insufficient as an independent quality metric. Reflectivity is affected by surface materials, orientation and surface complexity so this factor has been used to represent measurement quality. Given, however, that reflectivity is affected by multiple factors it, too, is insufficient as an independent quality metric.

The current state-of-the-art in quality metrics performs adequately in assessing the quality of measurements within the context of specific applications, but are often not readily generalizable. Few researchers combine quality metrics so that the strengths of one may offset the weakness of the other. More work is needed to develop a more comprehensive approach to measurement quality assessment. In the next chapter, we present this more comprehensive approach such that a high-quality model can be generated with minimal human intervention. This approach is presented within the context of using quality metrics to automate the data acquisition process. 


\section{Chapter 3}

\section{Region Mapping}

In this chapter, an approach to laser range scanning is presented in which quality metrics are used to automatically reduce the number of measurements acquired from a scanner viewpoint. As part of this approach, improved versions of the orientation and reflectivity quality metrics are presented, and three new quality metrics are introduced: outlier, resolvability, and planarity. These quality metrics are used to automatically divide the total field of view (TFoV) from a scanner viewpoint into three regions: the Unscannable region, the Completed region, and the Rescan region. The Unscannable region is likely to generate measurements that would be discarded as being of insufficient quality so is avoided during subscanning. The Completed region is likely to yield measurements that will add little new information to the surface model so is ignored during subscanning. The Rescan region is likely to yield measurements that contribute significant new information to the model so must be completely covered during subscanning. In order to ensure that the maximum amount of high-quality information is acquired from the current viewpoint, the operator is directed to move the scanner until the average quality-weighted spot size is minimized. A series of small high-density scans are then automatically generated to collect 
measurements from the Rescan region while minimizing coverage of the Unscannable region.

\subsection{Introduction}

Currently absent in the field of medium- to large-volume scanning is an interactive system capable of automatically obtaining a complete high-quality model of a scene or object in situ, using either an automated system or by guiding a minimally-trained operator through the scanning process, while minimizing the number of measurements acquired. Sequeira et al. used quality metrics for merging range images and, to a limited extent, for next-best-view (NBV) planning. Blais et al. iteratively merged multiple low-density scans until a stable model was achieved. Callieri et al. used a multi-stage approach, first developed by Scott et al. [110] for small-volume scanning, in which an initial lowdensity scan is followed by a series of high-density targeted scans. Region selection used by Callieri was, however, based solely on the measurability of each surface patch. In this chapter, the first two stages of a multi-stage approach are presented in which a series of quality metrics are used to adapt the scanning process such that the total quality of a composite range image is maximized while minimizing the number of measurements acquired. Unlike Callieri et al. [112], this approach uses the strengths of each quality metric, allowing the scanning process to be better tailored to the surface being scanned.

\subsubsection{Quality Metrics}

As discussed in Chapter 2, the quality of a range measurement depends on measurement uncertainty and measurement resolution which are strongly affected by environmental factors such as the type of surface material [57], surface reflectivity [77], distance to the surface [33] [77], incidence angle [75]. These environmental conditions must be detected in 
the data and combined with model-based uncertainty as metrics to better represent the quality of the virtual model. The quality of the mesh model used to represent the surface depends not only on the accuracy of each measurement, but also on how well the vertices and facets represent the surface. The few quality metrics that exist in contemporary literature have been described in Chapter 2, but all are limited in scope. They are often not used in conjunction with the physical properties and limitations of the scanner and/or surface. In this chapter, a low-density raster scan is used to perform a cursory examination of the environment, then various environmental factors are quantified using general-purpose quality metrics that relate to the physical properties of the scanner. These quality metrics are referred to as resolvability, planarity, orientation, reflectivity, and outlier quality metrics. These metrics are then used to both determine the quality of the measurements collected, and to direct the scanning process such that the potential quality of the resulting composite range image is maximized with respect to the scanner limits while minimizing total scan time.

The purpose of a quality metric is two-fold: it quantifies the relative position of some aspect of a range measurement on a continuum, and it quantifies the relationship of that aspect of a range measurement with respect to some previously established benchmark. It can then be used to compare methods or systems, or it can be used in an iterative process to maximize some aspect of a range image [54]. A quality metric uses two benchmarks to define the end points of the quality scale representing the best quality (1) and worst quality (0) associated with some attribute. Both benchmarks should be attainable, or nearly attainable, by the system. The best quality benchmark represents the attribute state which is most desired by the system so represents a target state. The worst-quality benchmark represents an unacceptable state so represents a breakpoint state. In all cases,

the quality metric has a value in the range $C_{i}^{\text {metric }}=1$ (ideal quality) to $C_{i}^{\text {metric }}=0$ (unacceptable quality) where metric refers to any metric presented in this chapter. 


\subsection{Laser Range Scanner System}

All range data was obtained using an auto-synchronizing variable-resolution triangulation laser range scanner designed by the National Research Council of Canada, shown in Figure 3.1. Figure 2.1 shows a schematic of the laser range scanner geometry. Note that the scanner in Figure 3.1 is rotated such that the $\mathrm{Y}$-axis mirror is oriented vertically rather than horizontally as shown in Figure 2.1. The scanner uses a green $(\lambda=532$ nanometres) laser that generates a laser spot with diameter 0.4 millimetres at the beam waist, 1.4 metres from the scanner. The spot expands to 0.9 millimetres at 1.0 and 1.8 metres from the scanner. The half-baseline length of the scanner is $h_{x}=0.045$ metres and the operational range is 0.5 metres to 10 metres [59]. The volume of interest (VoI) was limited to between 1 metre and 4 metres from the scanner for the experiments performed in this study.

The laser range scanning system consists of the laser range scanner (seen in Figure 3.1 , the scanner control workstation (SCW), and the model processing workstation (MPW). The SCW uses the QNX operating system to direct the operation of the laser range scanner and to convert peak and galvanometer (RAW) readings into Cartesian (XYZ) coordinates. RAW data conversion to XYZ is performed on the SCW after each raster scan and is stored as a text file. Range model generation is performed on the MPW using Matlab running under the Windows operating system. Matlab scripts issue commands via TCP/IP to the scanner control. After a sequence of one or more raster scans has been performed, all text files are copied to the MPW via TCP/IP. All data files transferred to the MPW are then converted into Matlab data files and pre-processed using the procedures described in Section 3.5.

The system is capable of performing raster and Lissajous scans within a TFoV of $30^{\circ} \times 30^{\circ}$ at between 128 and 4096 samples per scan line [59]; however, only raster scan patterns are used during the course of this study because they are what is employed most 


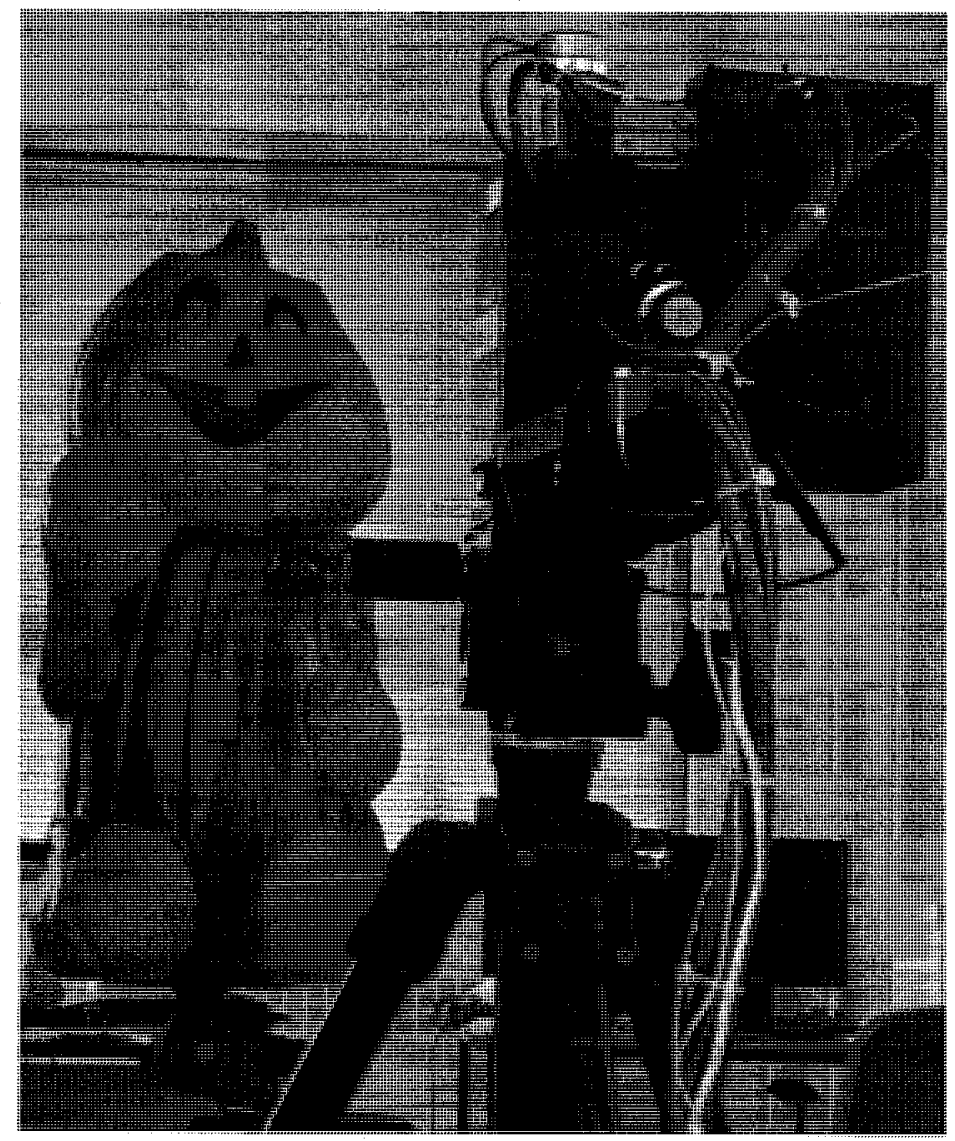

Figure 3.1: Autosynchronizing variable-resolution triangulation laser range scanner shown scanning the surface used in Experiment \#5. 
often in the field. Moreover, within each scan line the rotational separation between each measurement is constant because the scan velocity is constant, simplifying the process of determining the beam path in the environment. Raster scans are performed by the SCW by acquiring multiple scan lines of data, offsetting each scan line by a rotational increment. In all experiments, $256 \times 256$ raster scans are performed to minimize the size of the scan region. The Region of Interest ( $\mathrm{RoI})$ is bounded by scan line 3 and scan line 224, and by within-scan measurement 49 and within scan measurement 230 . The measurements on each scan line outside the RoI represent the time required to accelerate the beam to and from a full stop and to allow the scan velocity to stabilize, and are referred to as boundary measurements. All measurements, including boundary measurements, are retained throughout the region-building process but are discarded before generating the composite range image.

Not all measurements in a range image yield usable data; in some cases, the return signal is either insufficient to be detected (drop-out measurement) or exceeds the capacity of the photodetector (saturated measurement). In either case, the spatial and intensity measurement cannot be obtained so is generally assigned a value of zero. These measurements are referred to here as non-return measurements, while measurements that generate a non-zero range and intensity value are defined as return measurements. Nonreturn measurements are assigned the value $\hat{\mathbf{p}}=[0,0,0]$ by the SCW.

When calculating total scanning time, data transfer times have not been considered. The quantity of data being transferred is large while the data transfer rate for this system is low so data transfer between the SCW and MPW represents a significant bottleneck with respect to model generation time. The bottleneck could be significantly reduced in a commercial system so has not been considered important from the perspective of evaluating the performance of the model-building algorithm. For example, if the SCW and MPW were the same computer then the transfer time would be eliminated. The 
average time to perform each raster scan is approximately 10 seconds and conversion between RAW and XYZ on the SCW requires 30 seconds.

\subsubsection{Spherical Coordinate System}

All XYZ data are converted to the spherical (RTP) coordinate system to facilitate region generation and subscan generation where $\mathrm{R}$ refers to range, $\mathrm{T}$ refers to $\theta$ and $\mathrm{P}$ refers to $\phi$ as illustrated in Figure 3.2. The $\theta$ term refers to the horizontal rotation (along the scan line) and the $\phi$ term refers to the vertical rotation (between scan lines). The conversion equation is

$$
\left[\begin{array}{l}
R \\
\theta \\
\phi
\end{array}\right]=\left[\begin{array}{c}
\sqrt{x^{2}+y^{2}+z^{2}} \\
\tan ^{-1}\left(x / \sqrt{y^{2}+z^{2}}\right) \\
\tan ^{-1}(y / z)
\end{array}\right]
$$

where the range component $R$ is in units of metres, the rotational components, $\theta$ and $\phi$, are in units of radians, and $x, y$, and $z$ are in units of metres [11] [47] [113] [114]. Equation (3.1) is not the standard form of conversion between Cartesian and spherical but is used because it more closely approximates the geometry of the scanner as illustrated in Figure 2.1 .

Converting XYZ measurements to the RTP coordinate system makes it possible to treat the TFoV as a two-dimensional surface, bounded by the rotational limits of the galvanometers, in which each point has associated with it both a range and an intensity value. This surface is referred to as a region map and is used extensively in this study. Non-return measurements are assigned the value $\hat{\mathbf{p}}=[0,0,0]$ by the $\mathrm{SCW}$ so must be handled as a special case when translating XYZ coordinates to RTP coordinates for purposes of generating the region map. The region map is used to approximate the path of the laser through the VoI and experiments using surfaces of varying range verified that the $\theta$ and $\phi$ terms were approximately independant of range within the VoI. In order 


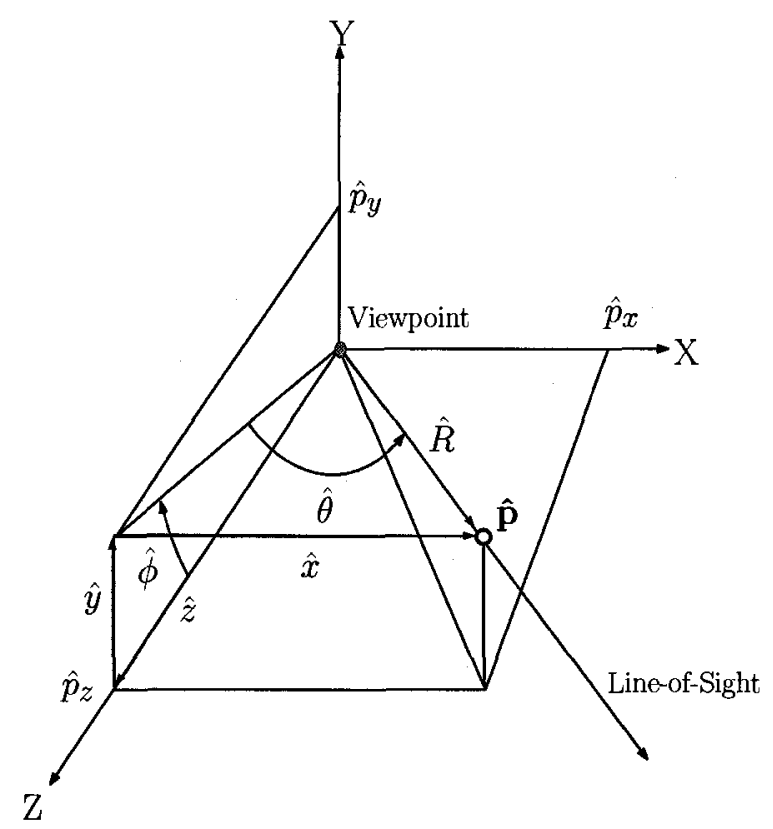

Figure 3.2: Orientation of a measurement $\hat{\mathbf{p}}$ with respect to the scanner origin (viewpoint). $\hat{\theta}$ is the rotational offset along the $(x, z)$-plane and $\hat{\phi}$ is the rotational offset from the $(x, z)$-plane toward the Y-axis. $\hat{R}$ is the distance along the line-of-sight to $\hat{\mathbf{p}}$. This format corresponds to the scanner geometry shown in Figure 2.1 in which $\hat{\phi}$ corresponds to motion of the $\mathrm{Y}$-axis scanner and $\hat{\theta}$ corresponds to motion of the $\mathrm{X}$-axis scanner. 
to approximate $\theta$ values for non-return measurements, the between-sample rotational distance within each scan is assumed to be constant and a simple linear equation is used to approximate all $\theta$ values for non-return measurements. Similarly, the between-scan line rotational distance is also assumed to be constant so that a linear equation is used to approximate all $\phi$ values for non-return measurements. The range and intensity values of all non-return measurements are assigned to be zero to indicate that they arise from a non-return measurement. The region map can, therefore, be represented as a regular grid of measurements that includes non-return measurements.

\subsection{Calibration}

Models were generated relating range, rotational, and intensity uncertainties, as well as a reference return signal intensity, to range values. Appendix $A$ shows the frequency histograms generated from 65,536 measurements at each of 8 distances from the laser range scanner between 0.8 and 3.6 metres. All measurements were obtained along the Z-axis of the scanner frame of reference. These data were then used to generate each of the models used in this study. In all cases, a flat white poster-board, oriented such that the surface normal was directed along the line-of-sight to the scanner, was used as a reference surface.

- Figure A-9 shows the best-fit curve relating range standard deviation to range values. The best-fit equation is

$$
\sigma_{R}(R)=70.25 \times 10^{-6} R^{3}-221.81 \times 10^{-6} R^{2}+268.08 \times 10^{-6} R-82.80 \times 10^{-6}
$$

- Figure A-10 shows the best-fit curves relating $\theta$ (horizontal rotation) and $\phi$ (vertical rotation) standard deviation to range values. The best-fit equations are

$$
\sigma_{\theta}(R)=97.71 \times 10^{-8} R^{-1}+740.55 \times 10^{-8}
$$


and

$$
\sigma_{\phi}(R)=11.24 \times 10^{-6}
$$

- Figure A-11 shows the best-fit curve relating expected return signal intensity to range values. The best-fit equation is

$$
I(R)=27.83 R^{-2}+288.79 R^{-1}-54.19
$$

- Figure A-12 shows the best-fit curve relating return signal intensity standard deviation to range values. The best-fit equation is

$$
\sigma_{I}(R)=-5.74 R^{-2}+10.04 R^{-1}-1.95
$$

It is interesting to note that $\sigma_{\theta}(R)$ increases with a decrease in range, particularly at less then 2.5 metres from the scanner origin. The vertical rotational uncertainty $\sigma_{\phi}(R)$ represents the between-scan line uncertainty so is relatively unaffected by parallax. Specifically, the length of the beam footprint along the vertical axis is relatively unchanged between 1 and 4 metres. On the other hand, the horizontal uncertainty $\sigma_{\theta}(R)$ is strongly affected by parallax, particularly for surfaces close to the scanner as illustrated in Figure A-10. In particular, given a surface with normal oriented along the line between the measurement and the scanner origin, the orientation of the laser beam increases with a decrease in range, resulting in an elongation of the beam footprint and, by extension, an increase in $\sigma_{\theta}(R)$. The $\sigma_{\theta}(R)$ term is reasonably constant between 2.5 and 4 metres, indicating that much of the range dependance of $\sigma_{\theta}(R)$ occurs close to the scanner as would be expected in this case.

Figure 3.3 summarizes the models used to generate range and intensity standard deviation, as well as laser spot radius, from range data. Figure 3.4 summarizes the models used to generate $\theta$ standard deviation and rotational resolution. Rotational resolution is 

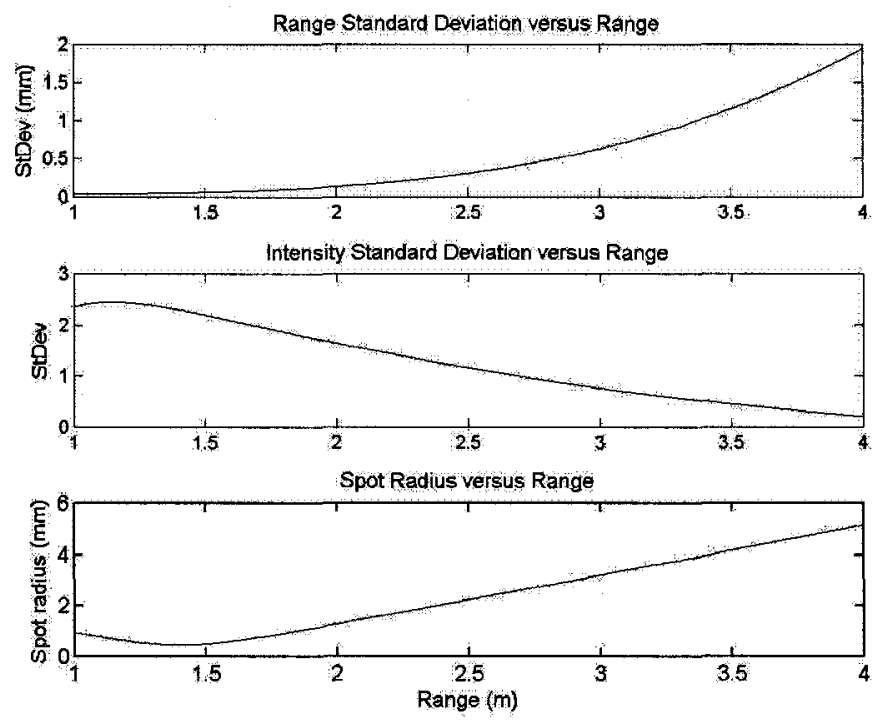

Figure 3.3: Summary of range models for range and intensity standard deviation, and for spot radius.
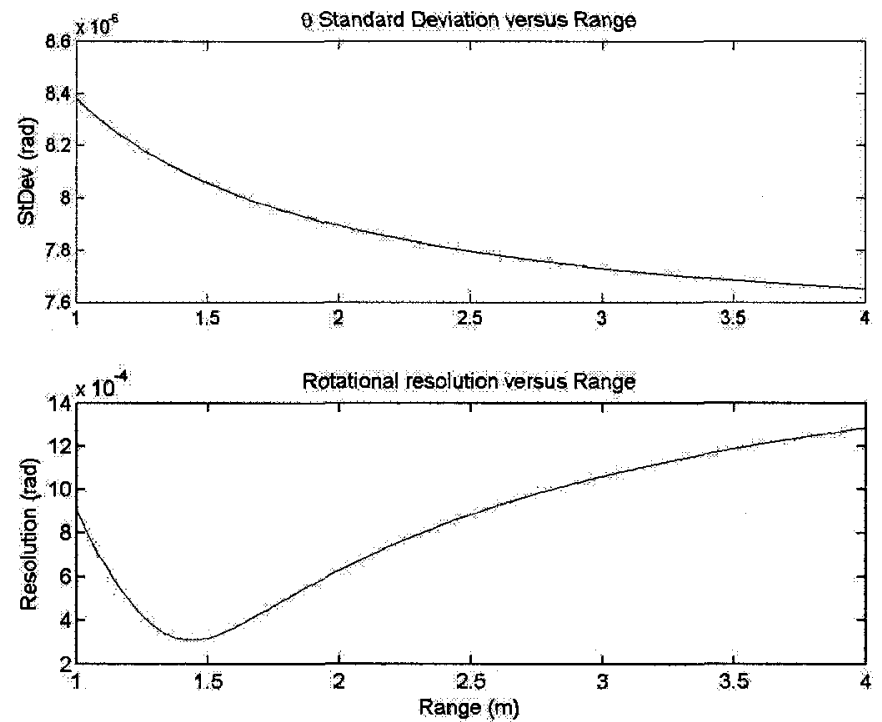

Figure 3.4: Summary of range models for $\theta$ standard deviation and for rotational resolution. In this case, rotational resolution refers to the arc-length of the laser spot in units of radians. 
the arc-length of the region covered by the laser spot expressed in units of radians. Specifically,

$$
\Delta \theta_{\text {res }}=\tan ^{-1}\left(\frac{\Delta d}{z}\right)
$$

where $\Delta \theta_{\text {res }}$ is the rotational resolution, $\Delta d$ is the radius of the laser spot, and $z$ is the distance between the laser spot and the scanner origin. At range values less than 1.4 metres the laser spot increases in diameter so its arc-length increases significantly. At ranges greater than 1.4 metres the laser spot also increases in diameter, but the increase is offset by (3.7). The term $\Delta \theta_{\text {res }}$ decreases as $z$ increases under constant $\Delta d$, but if the increase in $\Delta d$ is small as $z$ increases, then $\Delta d$ can, at sufficiently large $z$ be considered constant with respect to $\Delta d$. As a result, rotational resolution and spot size in the far field are generally treated as independant of range [33] [41] [90] [115]. This study, however, assumes surfaces in the mid-field so rotational resolution cannot be assumed independant of range.

\subsection{Test Surfaces}

Five test surfaces are used during the course of this investigation and are shown in Appendix B. The test surfaces consist of:

- A fiberglass head placed in front of the white poster-board used during calibration, referred to as Experiment \#1. Figure B-1 shows a view of the surface from the perspective of the laser range scanner. Figure B-2 shows a side view of the head to more clearly illustrate the surface structure.

- A section of wall with fine surface discontinuities, referred to as Experiment \#2. Figure B-3 shows the wall segment being scanned, illustrating that the surface is large enough to exceed the TFoV. Figure B-4 shows the surface details of the section of wall. 
- A plastic statue of a pumpkin man, referred to as Experiment \#3. Figure B-5 shows the plastic statue while Figure B-6 shows the region being scanned in detail.

- A ceramic statue placed in front of the white poster-board used during calibration, referred to as Experiment \#4. Figure B-7 shows the surface as seen from the laser range scanner. Figure B-8 is a side of the statue that illustrates the spatial complexity of the surface.

- A plastic foam-filled planter beside the white poster-board used during calibration, and a picture featuring four differently-coloured distributor caps, referred to as Experiment \#5. Figure B-9 shows a front view of the surface as seen from the laser range scanner. Figure B-10 shows the region being scanned in detail. Figure B-11 shows a detail view of the picture of the distributor caps with a reflective metallic surface in front of it. Figure B-12 shows the spatially complex surface of the plastic planter.

The test surfaces were selected to represent one or more issues that can arise during data acquisition as discussed in Chapter 2. These issues are summarized in Table 2.1 and Table 2.2. Specifically:

- Experiment \#1 represents a curved surface in front of a large planar surface. The poster-board is smaller than the TFoV so the boundaries of the poster-board should be detected as spatially complex regions while the planar inner surface should not be classified as spatially complex. The fiberglass head consists of a range of surface orientations so some surfaces should be detected as highly angled with respect to the laser path.

- Experiment \#2 represents a planar surface with fine surface discontinuities. The discontinuities are large enough to be detected by the scanner so should be identified as 
regions requiring dense scanning. Surface discontinuties should be flagged for highdensity scanning so that edge artefacts resulting from the spatial discontinuities can be examined in detail during post-processing.

- Experiment \#3 represents a complex curved surface that partially exceeds the TFoV. As can be seen in Figure B-6, the surface is reflective and consists of regions different reflectivity. The transitions between light and dark portions of the surface should be classified as intensity discontinuities and be isolated for high-density scanning. The surface features a variety of surface curvatures so highly oriented surfaces should be detected.

- Experiment \#4 represents a spatially complex surface with some planar surfaces. The statue represents a combination of fine details and smooth regions that should be isolated for dense scanning.

- Experiment \#5 represents a spatially complex surface with large planar surfaces. The planter, shown in Figure B-12, contains surface complexities that should be isolated for high-density scanning. Similarly, the picture of the distributor caps, shown in Figure B-11, consists of both range and intensity discontinuities that can result in both spatial and intensity measurement errors so should be isolated as regions requiring dense scanning. In particular, the intensity discontinuities on the picture of distributor caps should be flagged for high-density scanning so that edge artefacts resulting from the intensity discontinuities can be examined in detail during post-processing.

\subsection{Data smoothing}

Data obtained from a range scanner is typically smoothed to reduce the effect of noise. Often this process is performed by skilled operator who uses a combination of experience 
and personal preference to select a noise filter. In this study, much of the decision-making is being removed from the operator so it is useful to determine the optimal filter type and size. Once an optimal filter has been selected, it can be applied automatically. Noise filters are used to reduce the effects of random noise and are typically divided into linear and non-linear filters. Linear filters are employed to reduce random noise that follows a particular distribution such as Gaussian noise. Non-linear filters are used to eliminate random noise such as impulse noise.

Two popular linear filters are the local averaging filter and a Gaussian filter [116]. These filters smooth the data but may be affected by impulse noise and may blur edges. According to Trucco and Verri [117] an averaging filter will reduce Gaussian noise but will result in blurred edges, and it will diffuse, rather than remove, impulse noise. They warn that this could reduce the accuracy of results of edge localization. A Gaussian filter smoothes the data by replacing the central value with the weighted sum of the range values within the window [117]; however, it should attenuate, rather than remove, impulse noise [117]. In this study, edge detection is used to identify regions of interest so edge preservation is considered important. As a result, linear filters are not used in this study to perform data smoothing.

\subsubsection{Median Filtering}

The second class of filters is the non-linear filters, of which the median filter is a popular choice for removing outliers while minimizing the loss of edge information [118] [119]. Unweighted median filters provide better noise attenuation than weighted median filters [120] so only unweighted median filters are used in this study. A median filter smoothes the data by replacing the central value with the median of the range values within the window and is considered effective in smoothing a signal [121]. The resulting filtered signal features less blurring and isolated spikes are removed [119]. Under 
some circumstances the median filter can leave more noise than mean or Gaussian filter noise [122], and in the case of a recursive median filter that noise is cumulative [120]; however, median filters are still often recommended as a way to sharpen edges [123], and are considered to be better suited to handling impulse noise and preserving edges than linear filters [124]. The ability of the median filter to reduce noise depends upon finding a window size that is neither too large nor too small [125].

Median filters can be applied recursively to obtain a root signal within a finite time period [120] [126]. The root signal, or invariant [127] [128], is defined as a signal that does not change after further iterations of the filter. Such filters are referred to as idempotent because the signal generated by the filter is the root signal [120], and is considered to have better noise attenuation than the standard median filter [129]. For this reason, median filter iteration as well as filter window size are important factors in automating data pre-processing.

\subsubsection{Spot Size-dependant Median Filtering}

Median filtering is generally time-consuming because elements in each window must be sorted before the median value can be determined [130] [131]. This means that the filter window must be as small as possible, but must be large enough to ensure an appropriate level of smoothing. Typically, the optimal filter is determined through a combination of personal preference, experience, and post-scanning experimentation. The scanning method described in this thesis utilizes scan patterns of various densities so the filter window size that works well for one scan may not work well for another. In order to minimize the requirement for decisions by the user, the relationship between scan pattern coverage, instantaneous spot size, and smoothing must be established so that the filter window size can be adjusted automatically. 
A total of 8 scans were performed using a planar surface. The area covered by the scan pattern was increased after each scan, beginning with the smallest scan area that could be obtained and ending with an anchor scan. The XYZ data from each scan were converted to RTP, filtered using a square median filter of between $5 \times 5$ and $25 \times 25$, and the filter was applied between 1 and 5 times. The filtered RTP data were then converted to $\mathrm{XYZ}$ and fit to a regression plane. Appendix $\mathrm{C}$ shows the results of this experiment in which the size of the filter window (horizontal axis) and the number of repetitions (denoted by different lines) are correlated with the mean absolute residuals. The greater the mean absolute residuals the poorer the fit between the filtered data and the plane. The results are summarized in Figure 3.5 and Figure 3.6. Both use the term "Residualminimizing" to indicate that the curve represents the vertical axis value at which the residuals from a regression fit of the filtered data to a plane are minimized.

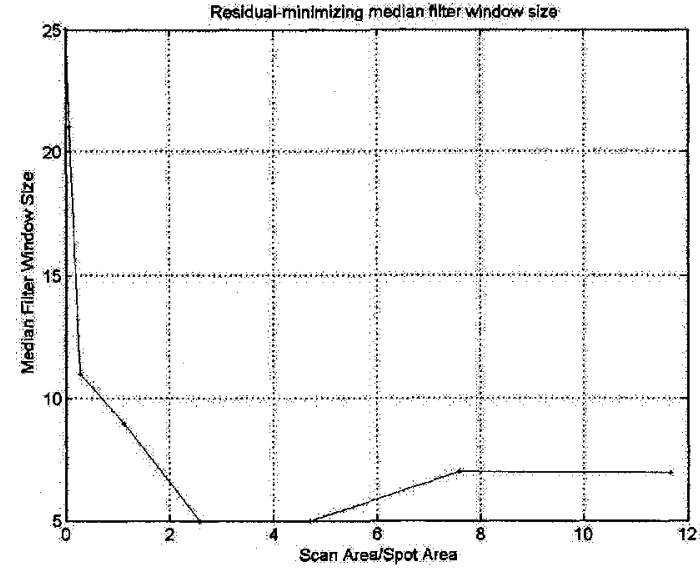

Figure 3.5: Median Window size.

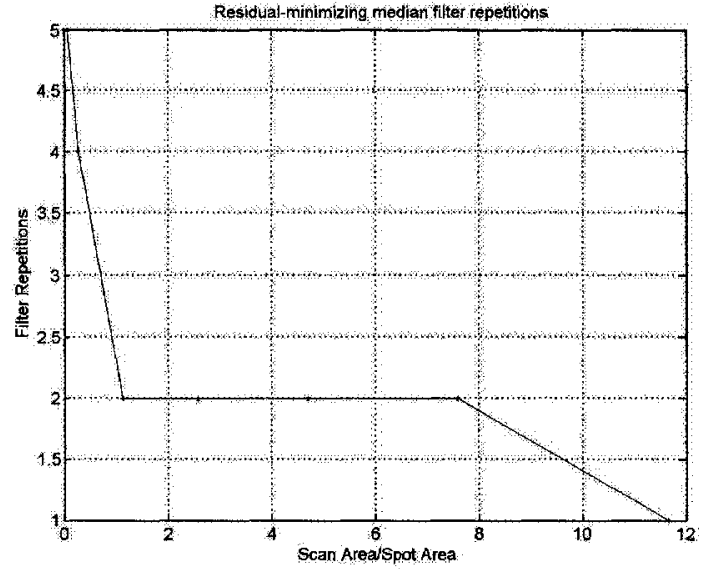

Figure 3.6: Median Window Repetitions.

The coverage of each scan pattern is defined by the relative rotational scan pattern per unit coverage of the instantaneous laser spots, referred to as the Scan Area/Spot Size 
in Appendix B. Given a rotational scan area per measurement defined by

$$
A_{\text {rot }}=4 \Delta \theta \Delta \phi
$$

where $\Delta \theta$ is the within-scan inter-sample distance and $\Delta \phi$ is the between-scan distance, and a spot area per measurement coverage defined by

$$
A_{\text {spot }}=\frac{\pi}{N_{\text {meas }}} \sum_{i=1}^{N_{\text {meas }}} \Delta \theta_{i}^{2}
$$

where $N_{\text {meas }}$ is the number of measurements and $\Delta \theta_{i}$ is the instantaneous rotational radius of the laser spot around $\hat{\mathbf{p}}_{i}$, the scan area to spot area ratio is defined by

$$
A_{\text {ratio }}=\frac{A_{\text {rot }}}{A_{\text {spot }}}
$$

When $A_{\text {ratio }}>1$, the scan pattern coverage is relatively large with respect to the instantaneous laser spot size. In this case, the inter-measurement rotational distance is, on average, larger than the instantaneous rotational radius of each laser spot. When $A_{\text {ratio }}<1$, the laser spots overlap so the inter-measurement rotational distance is smaller than the instantaneous rotational radius of each laser spot.

Figure 3.7 shows the relationship between integration distance and range measurement uncertainty. A large $\left(A_{\text {ratio }}>1\right)$ scan pattern has the benefit of an integration distance that is larger than the diameter of the laser spot so has a relatively low range uncertainty. The reduction in range noise is a result of the integration of the intensity signal over a sufficiently large region to minimize the effects of speckle noise. In Figure 3.7, large scan patterns correspond with $L / d_{o}$ values greater than 1 . A small $\left(A_{\text {ratio }}<1\right)$ scan pattern features an integration distance smaller than the diameter of the laser spot so correspond to $L / d_{o}$ values less than 1 . As a result, it is expected that $A_{\text {ratio }}=1$ should denote a transition between the requirements for filter window size, with smaller windows being sufficient for large $\left(A_{\text {ratio }}>1\right)$ scan patterns. This is confirmed in Figure 3.5 in 


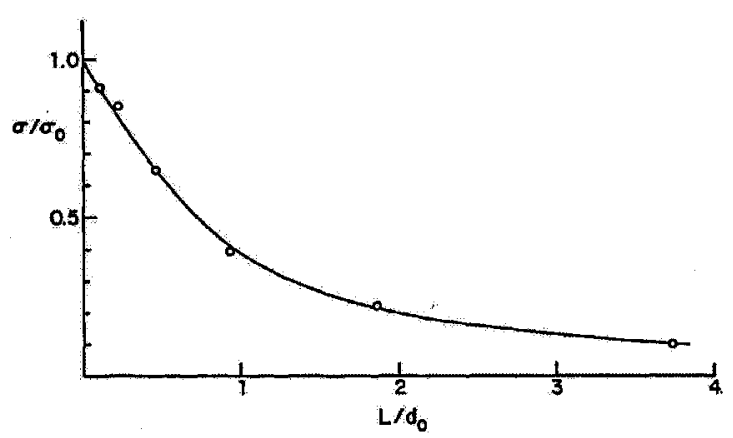

Figure 3.7: Increasing the distance $L$ between measurements per unit spot diameter $d_{0}$ results in a reduction in relative range measurement uncertainty $\sigma / \sigma_{0}$ where $\sigma_{0}$ is the range uncertainty without measurement integration (Reproduced from Figure 4 of [68]).

which $A_{\text {ratio }}>1$ corresponds with a median filter window size of between $5 \times 5$ and $7 \times 7$, while $A_{\text {ratio }}<1$ corresponds with a filter window of size $9 \times 9$ or greater.

From Figure 3.5 and Figure 3.6 it can be concluded that for large $\left(A_{\text {ratio }}>1\right)$ scan patterns, a median filter with window size no more than $7 \times 7$ elements should be applied using no more than two iterations to generate good smoothing of planar surfaces. In this study, scans with $A_{\text {ratio }}>1$ are smoothed using a $7 \times 7$ median filter applied with no iterations to the data set. Figure C-6 to Figure C-8 in Appendix B shows that a jump from 1 to 2 iterations of the median filter results in a noticable increase in fit error (vertical axis) to a planar surface. Meanwhile, for small $\left(A_{\text {ratio }}<=1\right)$ scan patterns, a median filter with window size no less than $9 \times 9$ elements should be applied using no less than two iterations to generate good smoothing of planar surfaces. In this study, scans with $A_{\text {ratio }} \leq 1$ are filtered using a $25 \times 25$ median filter applied twice to the data set.

Measurements at the edge of the RoI that have not been affected by the smoothing filter are discarded, and the RoI is reduced so that the data consists only of smoothed measurements. For example, if a $7 \times 7$ smoothing filter window is used, the first and last 3 
scan lines of the RoI, as well as the first and last 3 measurements of each scan line within the RoI are discarded and the RoI is reduced to bound the remaining measurements.

\subsubsection{Outlier Quality Metric}

The purpose of smoothing is to minimize the effects of sample noise; however, the postsmoothing spatial position and intensity of each measurement is a best guess based on the measurements within the filter window. Assuming the measurement range and intensity uncertainty models accurately reflect the possible distribution of measurements that could be obtained from the same point on the surface from the same scanner viewpoint, the best-guess replacement measurement should fall within $1.96 \sigma$ of the original measurement. Typically, $95 \%$ of the measurements should fall within $1.96 \sigma$ assuming the data is normally distributed with standard deviation $\sigma$ [132]. Best-guess measurements that do not fall within the expected error margin are suspect so are of lower quality than best-guess measurements within the expected error bound.

The outlier quality metric $C_{i}^{\text {out }}$ represents the reduction in confidence in a measurement after it has been shifted during typical pre-processing activities such as smoothing. For range measurements, the quality metric is found using

$$
C_{i}^{R, \text { out }}=\left\{\begin{array}{cc}
1 & \Delta R_{i}^{\text {shift }} \leq R_{i}^{\text {err }} \\
\frac{R_{i}^{\text {err }}}{\Delta R_{i}^{\text {shift }}} & \text { otherwise }
\end{array}\right.
$$

where $R_{i}^{\text {err }}=Z(\alpha / 2) \sigma_{R}$ is the two-tailed error margin assuming a normally-distributed data set [133] [134]. Typically $\alpha=0.05$ is used so $Z(\alpha / 2)=1.96$ [135], resulting in $R_{i}^{\text {err }}$ representing the $95 \%$ range error margin based on the range measurement uncertainty $\sigma_{R}$. The range measurement shift between pre- and post-smoothing is represented by $\Delta R_{i}^{\text {shift }}=\left|R_{i}^{\text {initial }}-R_{i}^{\text {final }}\right|$ where the range measurement prior to post-processing is $R_{i}^{i n i t i a l}$ and the range measurement after post-processing is $R_{i}^{f i n a l}$. For intensity measure- 
ments, the quality metric is found by

$$
C_{i}^{I, \text { out }}=\left\{\begin{array}{cc}
1 & \Delta I_{i}^{\text {shift }} \leq I_{i}^{\text {err }} \\
\frac{I_{i}^{\text {err }}}{\Delta I_{i}^{\text {shift }}} & \text { otherwise }
\end{array}\right.
$$

where $I_{i}^{e r r}=Z(\alpha / 2) \sigma_{I}$ is the two-tailed intensity error margin based on the intensity measurement uncertainty $\sigma_{I}$, and $\Delta I_{i}^{\text {shift }}=\left|I_{i}^{\text {initial }}-I_{i}^{\text {final }}\right|$. Once again, $Z(\alpha / 2)=1.96$ when $\alpha=0.05$. The intensity measurement prior to post-processing is $I_{i}^{\text {initial }}$ and the intensity measurement after post-processing is $I_{i}^{\text {final }}$. The outlier quality metric for the measurement $\mathbf{p}_{i}$ is the worst-case quality based on both range and intensity smoothing, and is found by

$$
C_{i}^{\text {out }}=\min \left\{C_{i}^{R, \text { out }}, C_{i}^{I, \text { out }}\right\}
$$

Because the outlier quality metric represents a deviation from what is expected based on the uncertainty model, $C_{i}^{\text {out }}$ is an augmentative quality metric; that is, measurements in which $C_{i}^{\text {out }}=0$ are still used in the final model, but measurements with higher $C_{i}^{\text {out }}$ are preferred.

Appendix D shows the outlier quality maps obtained from each of the five test surfaces, as well as the outlier quality map after applying a $7 \times 7$ median filter with no iteration. In all cases, the lightest-colour surfaces represent the worst quality measurements while the darkest-colour surfaces represent the best quality measurements. Regions corresponding to $C_{i}^{\text {out }}=1$ are considered ideal because neither the range nor intensity post-smoothing value differed from the original measurement by more than would be expected based on model-based range and intensity measurement uncertainty. Regions corresponding to $C_{i}^{\text {out }}=0$ represent non-return measurements because $C_{i}^{\text {out }} \in(0,1]$ for all return measurements. Regions corresponding to $C_{i}^{\text {out }} \rightarrow 0$ represent post-smoothing values that vary considerably from what would be expected based on model-based range and intensity measurement uncertainty. 
In Figure D-2, Figure D-8, and Figure D-10, the outlier quality decreases close to the transition points between return and non-return measurements because mixed measurements can result in range and intensity measurement errors. Changes in surface texture, as well as small changes in range measurements, can result in large changes in range or intensity values during the smoothing process when the window size is relatively large. For example, the recessed pattern on the wall segment in Experiment \#2, visible in Figure B-3, is just visible in Figure D-3. After applying the averaging filter, the recessed pattern is clearly seen as a region of low- $C_{i}^{\text {out }}$ measurements. The ability to detect such regions becomes important in Section 3.7.5 when selecting regions that should be rescanned.

\subsection{Anchor Scan Positioning}

It is important that the viewpoint from which the anchor scan is obtained be close enough to the object to maximize the quality of each measurement while ensuring that no surface is closer than the minimum acceptable range. In particular, the anchor scan should consist of as many measurements as possible that are resolvable to at least some target resolution $\Delta x$. Moreover, the scanner should be positioned to maximize the surface area oriented toward the scanner origin. On the other hand, the anchor scan should be obtained quickly and require minimal decision-making by the operator. These goals are achieved by performing a series of pre-anchor scans to find a viewpoint that locally minimizes the quality-weighted spot size using the predicted size of the laser spot on the surface. A gradient search is performed after each pre-anchor scan to predict a spatial position for the scanner origin that is likely to reduce the average quality-weighted spot size. The search space is restricted by the allowable range of scanner motion. In this section, the orientation quality metric and the resolvability quality metric are introduced, 
then they are used to find a locally-optimal scanner viewpoint from which to obtain an anchor scan.

\subsubsection{Orientation Quality Metric}

Surface orientation is a commonly-used quality metric [2] [67] [82] [88] [106] [107]; however, previous versions of this metric did not employ a maximum acceptable surface orientation as part of the metric. Surfaces with high orientation are generally discarded during post process because they result in low-quality measurements; however, the orientation quality metric $C_{i}^{\text {orient }}$ is defined to be

$$
C_{i}^{\text {orient }}=\left\{\begin{array}{cc}
0 & \cos \left(\gamma_{i}\right) \leq \cos \left(\gamma_{\max }\right) \\
\frac{\cos \left(\gamma_{i}\right)-\cos \left(\gamma_{\max }\right)}{1-\cos \left(\gamma_{\max }\right)} & \text { otherwise }
\end{array}\right.
$$

so that high orientation surfaces can be detected and eliminated before performing highdensity scanning [133] [134] [136] [137]. In (3.14), $\gamma_{i}$ is the orientation of the surface at point $\mathbf{p}_{i}$, defined using (2.24), and $\gamma_{\max }$ is the user-defined maximum acceptable orientation. Unlike contemporary orientation quality metrics, this approach generates a quality metric bounded by the target value $\cos \left(\gamma_{i}\right)=1$ and the breakpoint $\cos \left(\gamma_{i}\right)=$ $\cos \left(\gamma_{\max }\right)$. The orientation quality metric uses a pre-defined threshold $\gamma_{\max }$ so $C_{i}^{\text {orient }}$ is an exclusive quality metric; that is, measurements in which $C_{i}^{\text {orient }}=0$ are excluded from the final model.

Equation (3.14) can be further simplified by substituting (2.24) for $\cos \left(\gamma_{i}\right)$, eliminating the need to calculate the cosine values. As a result, (3.14) can be rewritten as

$$
C_{i}^{\text {orient }}=\left\{\begin{array}{cc}
0 & \vec{n}_{i}^{T} \hat{\mathbf{x}}_{i} \leq \vec{n}_{\max }^{T} \hat{\mathbf{x}}_{i} \\
\frac{\left(\vec{n}_{i}-\vec{n}_{\max }\right)^{T} \hat{\mathbf{x}}_{i}}{\hat{R}_{i}-\vec{n}_{\max }^{T} \hat{\mathbf{x}}_{i}} & \text { otherwise }
\end{array}\right.
$$

where $\hat{\mathbf{x}}_{i}$ is a measurement located $\hat{R}_{i}$ units from the viewpoint, $\vec{n}_{i}$ is the unit normal to the surface at $\hat{\mathbf{x}}_{i}$, and $\vec{n}_{\max }$ is the unit normal for a surface that is $\gamma_{\max }$ from the laser 
path. Measurements with $C_{i}^{\text {orient }}=0$ are classified as Angled, and arise from surfaces that are too highly angled so rescanning would yield measurements that would typically be discarded in post processing. Measurements with $C_{i}^{\text {orient }}>0$ are defined as Non-angled.

The orientation quality maps for the surfaces featured in Appendix B are shown in Appendix E. In all cases, $\gamma_{\max }=\pi / 4$ was used as the maximum acceptable surface orientation. Figure E-1, Figure E-7, and Figure E-9 feature large planar areas that show little variation in surface orientation. In Figure E-3 the recessed pattern is barely visible so is not detected when surface planarity is assessed in Section 3.7.1. Figure E-1, Figure E-5, Figure E-7, and Figure E-9 feature curved surfaces that are clearly visible in the orientation maps.

\subsubsection{Resolvability Quality Metric}

Prior to performing high-density scanning of a surface, it is useful to know whether it is even possible to achieve the desired surface resolution $\Delta x$ given the current scanner viewpoint. The resolution of any given measurement depends on the beam footprint, which is generally modelled using the laser spot area under the assumptions of a noncomplex surface that is continuous within the spot and with surface normal oriented along the laser path. For far-field measurements, the length of the scanner baseline is typically small enough that the scanner origin and laser origin can be assumed to be the same point; however, for near and mid-field measurements parallax can be a significant issue. In Figure 3.8(a), the surface orientation $\varrho_{i}$ is based on the deviation of the surface normal $\vec{n}_{i}$ from the line-of-sight to the laser origin. This coincides with the surface orientation used to calculate $C_{i}^{\text {orient }}$ only if the baseline is small enough compared to the distance from the scanner to the surface to be ignored. In all other cases, the calculation of $\varrho_{i}$ must be tailored to the geometry of the scanner. In this discussion, the term laser beam refers to the spatial region bounded by the laser, the surface, and the $1 / e^{2}$ boundary. 


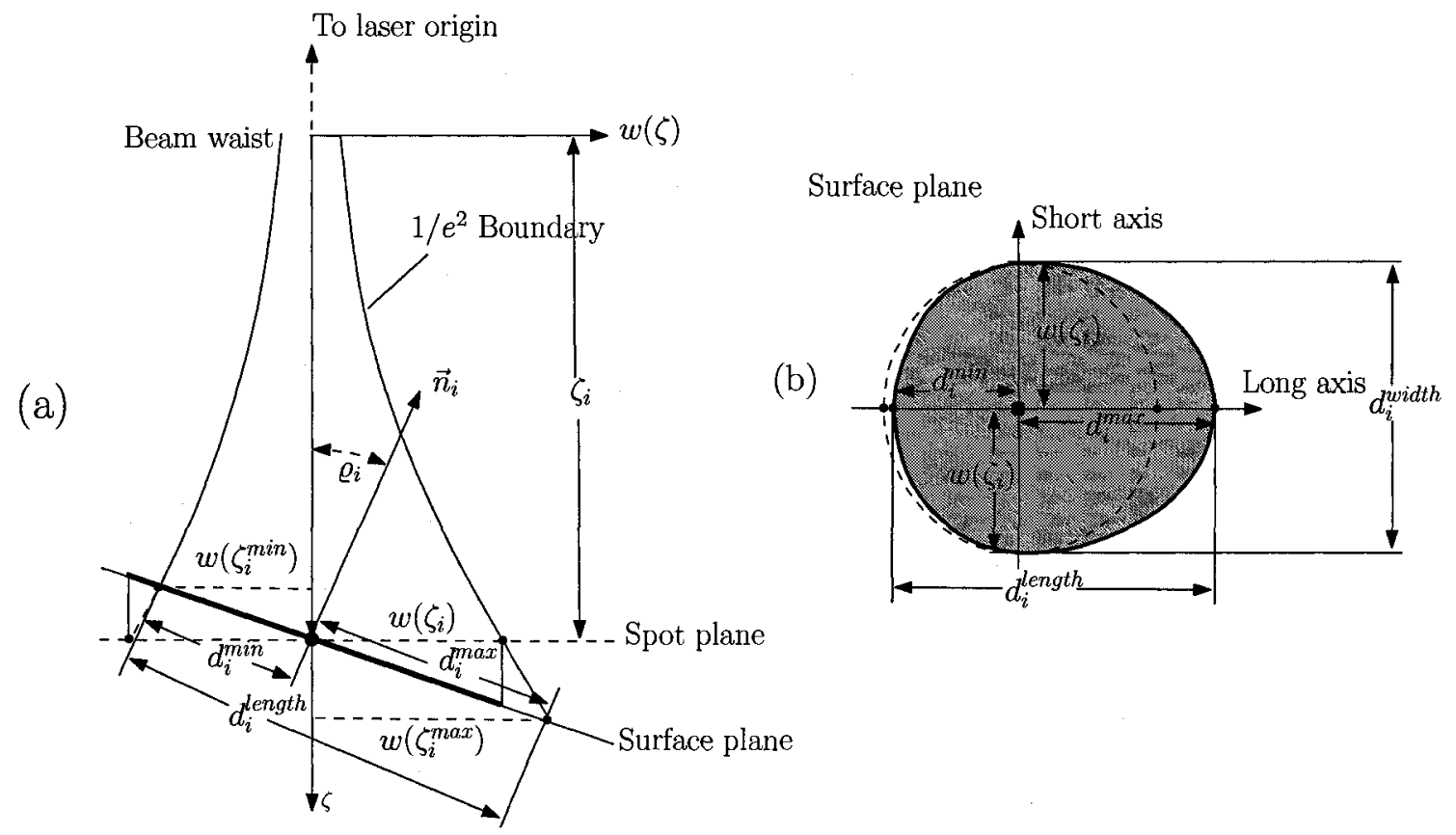

Figure 3.8: Beam intersection with an angled planar surface results in an oval beam footprint with length $d_{i}^{\text {max }}+d_{i}^{\text {min }}$ and width $2 w\left(\zeta_{i}\right)$. (a) side view with short axis directed outward from the page (b) top view with normal directed outward from the page. 
In Figure 3.8, the plane orthogonal to the beam path is the spot plane, represented by the dashed line in Figure 3.8(a), while the plane representing the approximate orientation of the surface is the surface plane, represented by the solid line in Figure 3.8(a). The surface resolution represents the resolution of the laser range scanner in the surface plane. This is rarely known a priori so the spot resolution, the resolution in the spot plane, is used to define the sampling density. Angulation of the surface with respect to the laser beam path can result in a distorted laser spot shape, as illustrated in Figure 3.8(b), making the determination of surface resolution more complex, particularly in the mid-field.

It is clear from Figure 3.8(a) that, particularly for mid-field measurements, the shape of the beam footprint resulting from the beam intersecting the surface plane can deviate significantly from the shape of the laser spot under the assumption that the surface normal is oriented along the laser path. This is more clearly represented in Figure 3.8(b) where the laser spot under the assumption that the surface normal is oriented long the laser path is represented by the circle bounded by dashed lines, and the beam footprint resulting from angulation of the surface is represented by the grey-filled oval bounded by thick lines.

The laser spot diameter on the spot plane is $2 w\left(\zeta_{i}\right)$, the length of the longest axis of the beam footprint and referred to as the beam footprint length, is $d_{i}^{\text {length }}=d_{i}^{\text {max }}+d_{i}^{\text {min }}$, while the beam footprint width is $d_{i}^{\text {width }}=2 w\left(\zeta_{i}\right)$, the same as the diameter of the laser spot. The longest segment $d_{i}^{\text {max }}$ and shortest segment $d_{i}^{\text {min }}$ of the long axis of the beam footprint, assuming a planar surface that is continuous within the $1 / e^{2}$ region, can be found by

$$
\left\{d_{i}^{\min }, d_{i}^{\max }\right\}=\left|\frac{-K_{i}^{2} \pm \sqrt{\left(K_{i}^{2}\right)^{2}-4 K_{i}^{1} K_{i}^{3}}}{2 K_{i}^{1}}\right|
$$


where

$$
\begin{aligned}
K_{i}^{1} & =\left[w(0) \sin \left(\varrho_{i}\right)\right]^{2}-\left[\zeta_{0} \cos \left(\varrho_{i}\right)\right]^{2} \\
K_{i}^{2} & =2 \zeta_{i} w^{2}(0) \sin \left(\varrho_{i}\right) \\
K_{i}^{3} & =\left[w(0) \zeta_{0}\right]^{2}+\left[w(0) \zeta_{i}\right]^{2}
\end{aligned}
$$

and $\zeta_{i}$ is the distance between the beam waist and the intersection of the beam peak with the surface [133] [136] [137]. These results hold even through the beam waist because the $1 / e^{2}$ boundary is symmetrical about $\zeta=0$. The derivation of $(3.16)$ can be found in Appendix F. In the near and far field, $d_{i}^{\text {length }}$ is simply approximated from the longest axis of the intersection of a plane with a cone.

The resolvability quality metric $C_{i}^{\text {resolve }}$ is used to identify regions that cannot be resolved at the desired surface resolution $\Delta x$ given the current scanner viewpoint to within a margin of error. This metric is found by

$$
C_{i}^{\text {resolve }}=\left\{\begin{array}{cc}
1 & d_{i}^{\text {length }} \leq d_{i}^{\text {upper }} \\
\frac{d_{i}^{\text {upper }}-d_{i}^{\text {width }}}{d_{i}^{\text {length }}-d_{i}^{\text {width }}} & d_{i}^{\text {width }} \leq d^{\text {upper }}<d_{i}^{\text {length }} \\
0 & \text { otherwise }
\end{array}\right.
$$

where $d_{i}^{\text {length }}$ is the length of the long axis of the beam footprint, $d_{i}^{\text {width }}$ is the length of the short axis, and $d_{i}^{u p p e r}=\Delta x+2 d_{i}^{\text {err }}$ is the desired surface resolution with an error margin based on the measurement rotational uncertainty [133] [134] [137]. If $d_{i}^{\text {length }}=d_{i}^{\text {width }}$, such as when $\gamma_{i}=0$, then $d_{i}^{\text {width }} \leq d^{\text {upper }}<d_{i}^{\text {length }}$ cannot occur and (3.18) becomes a binary equation such that $C_{i}^{\text {resolve }}=1 \mathrm{iff} d_{i}^{\text {length }} \leq d_{i}^{\text {upper }}$, otherwise $C_{i}^{\text {resolve }}=0$. The resolvability quality metric uses a pre-defined threshold $\Delta x$ so $C_{i}^{\text {resolve }}$ is, like $C_{i}^{\text {orient }}$, an exclusive quality metric.

The error term $d_{i}^{\text {err }}$ is added to the radial distance from the centre to the edge of the beam footprint to ensure the scanner is close enough to resolve features to at least $\Delta x$ with a margin of error. This error term is found using

$$
d_{i}^{e r r}=\frac{R \sin \left(\theta_{e r r}\right)}{\cos \left(\gamma_{i}\right)-\sin \left(\gamma_{i}\right) \sin \left(\theta_{e r r}\right)}
$$


where $\gamma_{i}$ is the angle between the surface normal $\vec{n}_{i}$ and the laser path $\vec{r}_{i}$ [137]. The rotational error term $\theta_{\text {err }}$ is defined as

$$
\theta_{\text {err }}=\sqrt{\chi^{2}(1, \alpha)\left(\max \left\{\sigma_{\theta}^{2}, \sigma_{\phi}^{2}\right\}\right)}
$$

where $\chi^{2}(1, \alpha) \approx 3.84$ for $\alpha=0.05$ [138]. The $\theta_{\text {err }}$ term represents the maximum distance between two points, in this case the centre of one beam footprint and the edge of its neighbouring beam footprint, such that they can still be considered likely to represent the same point to within a $95 \%$ confidence level given the expected rotational uncertainty of the scanner [137].

Appendix E shows the resolvability quality maps for the surfaces shown in Appendix B. For planar surfaces, such as those in Figure E-2, Figure E-4, Figure E-8, and Figure E-10, resolvability quality decreases the farther one travels from the point on the planar surface closest to the scanner. All angled measurements have $C_{i}^{\text {resolve }}=0$ because such surfaces will have very large $d_{i}^{\text {length }}: d_{i}^{\text {width }}$ ratios, resulting in $C_{i}^{\text {resolve }} \rightarrow 0$.

\subsubsection{Quality-weighted Spot Size}

Ideally, the scanner should be close enough to the surface that most of the measurements will be obtained using laser spots of minimal size; however, that must be balanced by the need to maximize the number of measurements obtained that will not later be discarded. The orientation and resolvability metrics are exclusive quality metrics so can be used to predict which measurements from a given viewpoint are likely to be discarded. The quality-weighted spot size $W_{i}^{s p o t}$ of each measurement is defined by

$$
W_{i}^{\text {spot }}=w\left(\zeta_{i}\right)\left(1-C_{i}^{\text {orient }} C_{i}^{\text {resolve }}\right)
$$

where $w\left(\zeta_{i}\right)$ is the radius of the laser spot assuming the surface normal is oriented along the laser path, $\zeta_{i}$ is the distance to the beam waist, $C_{i}^{\text {orient }} \in[0,1]$ is the orientation quality metric, and $C_{i}^{\text {resolve }} \in[0,1]$ is the resolvability quality metric [133] [134] [137]. 
The volume bounded by $w\left(\zeta_{i}\right)$ represents the region within which $86.5 \%$ of the beam irradiance is contained [89]-[91]. The quality-weighted spot size for each measurement is minimized when $w\left(\zeta_{i}\right)$ is minimized and when the exclusive quality metric product $C_{i}^{\text {orient }} C_{i}^{\text {resolve }}$ is maximized.

The average quality-weighted spot size is found by

$$
W_{\text {spot }}=\frac{\sum_{i=1}^{N_{\text {return }}} W_{i}^{\text {spot }}}{W_{\text {waist }}^{\text {spot }} N_{\text {return }}}
$$

where $N_{\text {return }}$ is the number of return measurements and $W_{\text {waist }}^{\text {spot }}$ is the size of the laser spot at the beam waist. The term $W_{\text {waist }}^{\text {spot }}$ acts as a scaling constant.

\subsubsection{Scanner Positioning}

Scanner positioning involves obtaining the average quality-weighted spot size $W_{\text {spot }}$ for the current scanner viewpoint, then virtually moving the scanner viewpoint in a direction that results in a decrease in $\tilde{W}_{\text {spot }}$ where $\tilde{W}_{\text {spot }}$ is the expected average quality-weighted spot size at the new virtual scanner viewpoint. If moving the scanner results in $\Delta W_{\text {spot }}=$ $W_{\text {spot }}-\tilde{W}_{\text {spot }}$ greater than some threshold value $T_{\text {spot }}$, or the repositioning required to achieve $\tilde{W}_{\text {spot }}$ is sufficiently large, then the operator moves the scanner, performs another anchor scan, and recalculates both $W_{\text {spot }}$ and $\tilde{W}_{\text {spot }}$. In this study, the process of respositioning the scanner and reacquiring the anchor scan was terminated when either $\Delta W_{\text {spot }}<T_{\text {spot }}$ where $T_{\text {spot }}=1 \times 10^{-6}$, or the requested translation was no more than 1 centimetre and the requested rotation was no more than $5^{\circ}(\approx 0.03 \pi)$. The value for $T_{\text {spot }}$ was experimentally derived, while the translation and rotation limits represented the practically-achievable minimum positioning accuracy of the operator given the constraints of the scanning system.

Appendix $\mathrm{G}$ shows the results of performing iterative anchor scan position re-adjustments of each of the test surfaces shown in Appendix B. In all cases, scanner motion was 
limited to $\mathrm{X}$-axis and $\mathrm{Z}$-axis translation, as well as a horizontal and vertical rotation about the new scanner position. Table G-1, Table G-2, Table G-3, Table G-4, and Table G-5 show the measured initial quality-weighted spot sizes before repositioning, as well as the predicted new quality-weighted spot size after moving the scanner in the prescribed manner. Each table also shows the translational and rotational motion information provided to the operator.

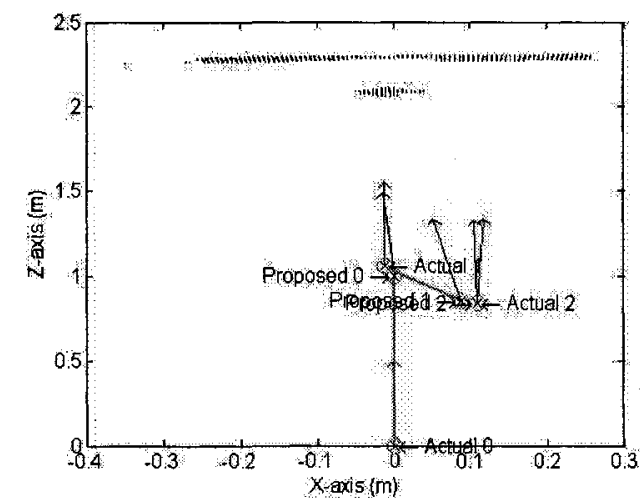

Figure 3.9: Experiment \#1 Viewpoint Trajectory.

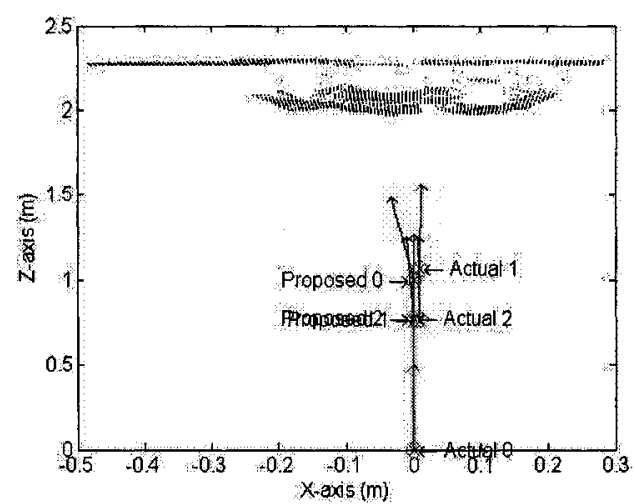

Figure 3.10: Experiment \#4 Viewpoint Trajectory.

Figure 3.9 summarizes the changes in scanner viewpoint relative to the surface used in Experiment \#1. The graphical instructions provided to the operator prior to each viewpoint position and orientation change in Experiment \#1 can be seen in Figure G-1, Figure G-2, and Figure G-3. Similarly, Figure 3.10 summarizes the viewpoint position and orientation changes for Experiment \#4. The points marked "Actual" represent the actual position of the scanner with respect to the surface. The points marked "Proposed" represent the location to which the operator is requested to move the scanner. The arrows represent the scanner orientation. In all experiments an optimal position was achieved within relatively few (no more than three) viewpoint adjustments. 


\subsection{Region-based Adaptive Scanning}

Region-based adaptive scanning consists of extracting the regions of the TFoV that correspond to the surface of interest, then scanning only those regions likely to contribute useful and non-redundant information to the model of the object being scanned. A region map is generated from the anchor scan in which the horizontal and vertical axes represent the rotational extents of the laser range scanner. The region map is then analyzed to identify the portions of the $\mathrm{TFoV}$ in which rescanning with one or more targeted dense raster scans would likely result in an improvement in the quality of the composite range image, referred to here as the Rescan region, and which portions are likely to yield either redundant or unacceptable measurements. The region likely to yield measurements that will add little new information to the composite range image is referred to as the Complete region, while the region likely to yield measurements that will be discarded is referred to as the Unscannable region. Region generation is based not only on the orientation, resolvability, and outlier quality metrics, but also on the surface planarity and the surface reflectivity. In this section, the planarity and reflectivity quality metrics are introduced, then they are combined with the other quality metrics as part of the region-generation process.

\subsubsection{Planar Quality Metric}

One way to extract regions requiring high-density scans from the total field of view is to identify regions containing spatially complex surfaces. A spatially complex surface features range measurements that change rapidly within the resolution of the system and require sampling at the maximum density of the scanning system to ensure that the surface is accurately represented in the composite range image. Rather than search for spatial complexity, the Rescan region is initially assumed to consist of spatially complex surfaces. Planar surfaces are then identified and used to initialize the Complete region. 
Subsampling planar regions is likely to yield little new information so the Complete region can be ignored during subscanning.

Planar surfaces are defined by all measurements in which the planarity quality metric is $C_{i}^{\text {planar }}=1$. A measurement $\hat{\mathbf{p}}_{i}$ that is contained within a locally planar neighbourhood is assigned $C_{i}^{\text {planar }}=1$; otherwise, it is assigned $C_{i}^{\text {planar }}=0$. Stamos [139] defined planar measurements using two criteria:

1. all measurements in a neighbourhood fit a regression plane to within a pre-defined margin of error; and

2. the regression planes associated with all measurements in a neighbourhood are oriented in the same direction.

The second criteria is ensured by requiring that the unit normals $\vec{n}_{i, j}$ of the surfaces associated with all measurements in the neighbourhood not deviate from each other by more than a threshold amount $T_{\text {normal }}$. In this study, the surface unit normal $\vec{n}_{i}$ associated with $\hat{\mathbf{p}}_{i}$ is obtained by determining the unit normal of the best-fit regression plane of the 8-neighbourhood of $\hat{\mathbf{p}}_{i}$.

Once the surface unit normals have been calculated for all measurements, the deflection between each 8-neighbour $\hat{\mathbf{p}}_{i, j}$ of $\hat{\mathbf{p}}_{i}$ is calculated using

$$
\cos \left(\gamma_{i, j}\right)=\vec{n}_{i}^{T} \vec{n}_{i, j}
$$

where $\vec{n}_{i}$ is the unit normal of the best-fit regression plane for the 8-neighbourhood of $\hat{\mathbf{p}}_{i}$, and $\vec{n}_{i, j}$ is the unit normal of the best-fit regression plane for the 8-neighbourhood of $\hat{\mathbf{p}}_{i, j}$. If the 8-neighbourhood of $\hat{\mathbf{p}}_{i}$ is completly planar then $\cos \left(\gamma_{i, j}\right)=1$ for all 8-neighbours of $\hat{\mathbf{p}}_{i}$. In this study, the 8-neighbourhood is considered to be sufficiently planar if $\cos \left(\gamma_{i, j}\right)>$ $T_{n o r m a l}$ for all 8-neighbours of $\hat{\mathbf{p}}_{i}$. In this study, a threshold value of $T_{\text {normal }}=\cos (0.083 \pi)$ was selected based on experimentation. The value of $T_{\text {normal }}$ can be adjusted by the 
operator or established as part of the calibration process to adjust how much of the surface is included in the planar region. When a measurement $\hat{\mathbf{p}}_{i}$ is referred to as planar, this means that the measurement is locally coplanar within its 8-neighbourhood. The planarity quality metric represents a deviation from the expectation of a planar surface, so $C_{i}^{\text {planar }}$ is, like $C_{i}^{\text {out }}$, an augmentative quality metric.

Appendix $\mathrm{H}$ shows the planarity quality map of all surfaces featured in Appendix B. Figure H-1 and Figure H-9 illustrate that the orientation threshold is sufficiently relaxed that even curved surfaces are classified as being planar. The planarity metric is used to identify spatially complex surfaces so simple surfaces like shallow curves are acceptable. Figure H-1, Figure H-5, Figure H-13, and Figure H-15 contain large planar surfaces that are correctly identified; however, the recessed pattern in Figure H-5 is not detected. Increasing $T_{\text {normal }}$ could solve the problem but at the cost of incorrectly labelling some other planar surfaces as being spatially complex. More pronounced surface features, such as those seen in Figure H-1 and Figure H-9, are detected.

\subsubsection{Reflectivity Quality Metric}

As discussed in Section 2.4.1, the qualitative effects of return signal intensity can be more easily examined when return signal intensity has been converted to reflectivity. In Table 2.2 , range and surface reflectivity changes have been noted as environmental factors that can affect return signal intensity. Reflectivity is calculated by dividing the observed return signal intensity by the expected return signal intensity of a reference surface. Specifically,

$$
\rho_{i}=\frac{\hat{I}_{i}}{I\left(\hat{R}_{i}\right)}
$$

is the surface reflectivity $\rho_{i}$ associated with a measurement $\hat{\mathbf{p}}_{i}$ relative to the return intensity model $I\left(\hat{R}_{i}\right)$ based on the reference surface defined by (3.5). Changes in return signal intensity could be due to changes in surface reflectivity or differences in distance 
from the scanner to the surface. Equation (3.24) removes the effect of range on the return signal intensity so that changes in reflectivity should only be due to differences in surface qualities such as orientation or changes in surface material. As indicated in Section 3.6.1, a reduction in surface reflectivity can increase measurement uncertainty so any deviation from the reference represents a potential change in real measurement uncertainty from that predicted by the model.

Fiocco et al. [88] had previously defined a reflectivity quality metric as (2.11) which is a binary quality metric; however, this reduces the generalizability of the metric. The reflectivity quality metric $C_{i}^{\text {reflect }}$ is defined to be

$$
C_{i}^{\text {reflect }}=\left\{\begin{array}{cc}
0 & \rho_{i} \geq \rho_{\max } \\
\frac{\rho_{\max }-\rho_{i}}{\rho_{\max }-1} & \rho_{\max }>\rho_{i}>1 \\
1 & \rho_{i}=1 \\
\frac{\rho_{i}-\rho_{\min }}{1-\rho_{\min }} & \rho_{\min }<\rho_{i}<1 \\
0 & \rho_{i} \leq \rho_{\min }
\end{array}\right.
$$

where $\rho_{\min }$ and $\rho_{\max }$ are user-defined bounds on the acceptable reflectivity of the surface [133] [134] [136] [137]. The reflectivity quality metric, like $C_{i}^{\text {orient }}$ and $C_{i}^{\text {resolve }}$, uses pre-defined thresholds $\rho_{\max }$ and $\rho_{\min }$ so $C_{i}^{\text {reflect }}$ is an exclusive quality metric.

Appendix $\mathrm{H}$ shows the reflectivity quality map of all surfaces featured in Appendix B. Figure H-2, Figure H-14, and Figure H-18 illustrate the effect of different surface materials on surface reflectivity. In particular, the changes in surface colour in Figure H-18 illustrate how even materials like paint can significantly change surface reflectance properties. Sudden changes in surface reflectivity, as illustrated in Figure 2.8, can result in range measurement errors by changing the shape of the return signal profile. As a result, regions containing sudden transitions in the reflectivity profile should be identified and moved from the Complete region to the Rescan region. 


\subsubsection{Reflectivity-based Edges}

In this study, reflectivity changes were detected within each neighbourhood using a Sobel edge filter [140] applied to the reflectivity quality metric. Planar measurements in which the reflectivity edge strength exceeded an experimentally-derived value of 0.1 were classified as edges so that they would be moved from the Complete region to the Rescan region. The Complete region is initially composed of planar measurements, so for purposes of region-building, planar measurements that are also edge measurements in the reflectivity map are reclassified as non-planar measurements.

Appendix $\mathrm{H}$ shows the planarity quality map of all surfaces featured in Appendix $\mathrm{B}$ after detecting reflectivity-based edges. In Figure H-7, Figure H-11, and Figure H19, sudden changes in surface reflectivity due to surface gloss (Figure H-11), changes in material properties (Figure H-11) and changes in surface colour (Figure H-19) were detected and used to increase the coverage of the Rescan region.

\subsubsection{Low-Outlier Detection}

Post-smoothed measurements that deviate significantly from the pre-smoothed values may indicate that the surface has not been sampled at sufficient density to detect all surface features, or that the surface material could generate more noisy measurements. In either case, regions containing such measurements are moved from the Complete region to the Rescan region for more detailed analysis. As with reflectivity edges, this is accomplished by reclassifying planar measurements with $\tilde{C}_{i}^{\text {outlier }}<C_{\text {threshold }}^{\text {outlier }}$ as nonplanar where $\tilde{C}_{i}^{\text {outlier }}$ is the outlier metric after applying a $7 \times 7$ averaging filter [117]. The averaging filter is used to attenuate and diffuse the high-frequency components of the outlier quality map. As a result, only regions with dense low- $C_{i}^{\text {outlier }}$ measurements are isolated while regions with sparse low- $C_{i}^{\text {outlier }}$ measurements are ignored. 
Appendix $\mathrm{H}$ shows the planarity quality map of all surfaces featured in Appendix B after low-outlier measurements are detected. The smoothed outlier threshold was defined to be $C_{\text {threshold }}^{\text {outlier }}=0.5$ for the anchor scan and $C_{\text {threshold }}^{\text {outlier }}=0.4$ for all subscans. The larger threshold for the anchor scan represents the lower tolerance for measurement uncertainty when selecting regions for rescanning. Glossy surfaces (Figure H-12), spatially complex surfaces (Figure H-16 and Figure H-20), and surfaces made with materials that are poor reflectors (Figure $\mathrm{H}-4$ and Figure $\mathrm{H}-8$ ) are detected. In particular, the recessed pattern in Figure $\mathrm{H}-8$ is accurately detected using the outlier metric and is correctly moved from the Complete region to the Rescan region. The detection of glossy and poorly reflective surfaces is important so that the transition between non-glossy/adequately reflective surfaces and glossy/poorly reflective surfaces can be better defined.

\subsubsection{Region Mapping}

Once the anchor scan has been acquired, a Region map is generated using Delaunay triangulation. Facets in which all vertices are non-return measurements are used to generate the Unscannable region. This approach means that the Rescan region covers the transition between return and non-return measurements, what is referred to as the silhouette of the surface. The Rescan map does not include the transition between the edge of the TFoV and the Complete region, what is referred to as the TFoV boundary; the Complete region is assumed to continue beyond the edge of the TFoV boundary.

The Unscannable region is initially defined by all non-return measurements, then is expanded by including measurements in which $C_{i}^{\text {exclusive }}=0$ where the within-scan exclusive quality metric is defined by the product of the exclusive quality metrics. Specifically,

$$
C_{i}^{\text {exclusive }}=C_{i}^{\text {reflect }} C_{i}^{\text {resolve }} C_{i}^{\text {orient }}
$$

where $C_{i}^{\text {reflect }}, C_{i}^{\text {resolve }}$, and $C_{i}^{\text {orient }}$ were previously identified as exclusive quality metrics [133] [134]. Specifically, any facet in which at least one vertex has $C_{i}^{\text {exclusive }}=0$ 
are moved to the Unscannable region. Once again, this ensures that the Rescan region includes the transition between Angled and Non-angled surfaces, Resolvable and Unresolvable surfaces, and Reflective and Non-reflective surfaces. Resolvable surfaces are defined as surfaces that generate measurements with $C_{i}^{\text {resolve }}>0$, while unresolvable surfaces are defined as surfaces that generate measurements with $C_{i}^{\text {resolve }}=0$. Reflective surfaces are defined as surfaces that generate measurements with $C_{i}^{\text {reflect }}>0$, while Nonreflective surfaces are defined as surfaces that generate measurements with $C_{i}^{\text {reflect }}=0$.

The Complete region is defined by all facets in which all the vertices have $C_{i}^{\text {planar }}=1$ after reflectivity edges and low-outlier measurements have been used to reclassify formerly planar measurements into non-planar measurements. The facets remaining in the Rescan region become the target for high-density rescanning using multiple small raster scans. The final region map for each of the surfaces featured in Appendix B can be found in Appendix I. White areas represent the Unscannable region while dark areas represent the Rescan region. The remainder of each map consists of Complete region.

\subsection{Subscan Generation}

A series of dense $256 \times 256$ subscans is automatically generated such that the intersample separation maximizes the likelihood of achieving a predefined target resolution $\Delta x$, while both minimizing the number of subscans obtained and ensuring complete coverage of the Rescan region. The size of the subscan was selected as the smallest scan size that could practically be generated by the scanner. The inter-sample separation can be defined using either of two approaches, depending on the needs of the operator: the target-based approach or the spot-based approach. In both approaches, the Rescan region is initially bounded by a box, then the box is subdivided into subscan regions such that all parts of the Rescan region are covered. The subscans are then shifted such that the coverage of the Unscannable region is minimized and coverage of the Rescan region is 
guaranteed to be complete. Each subscan is shifted to err on the side of rescanning a portion of the Complete region over rescanning a portion of the Unscannable region because it is preferable to retain measurements that may be redundant rather than to collect measurements that are likely to be discarded.

In the target-based approach, the inter-sample distance is defined by the target surface resolution after considering surface orientation. The target-based inter-sample separation $\theta^{\text {target }}$ is defined by

$$
\theta^{\text {target }}=\min _{i=1}^{N}\left\{\theta_{i}^{\Delta x}-\theta_{i}^{\text {err }}\right\}
$$

where $\theta_{i}^{\Delta x}$, found using

$$
\theta_{i}^{\Delta x}=\tan ^{-1}\left(\frac{\frac{\Delta x}{2} \cos \left(\gamma_{i}\right)}{\hat{R}_{i}+\frac{\Delta x}{2} \sin \left(\gamma_{i}\right)}\right)
$$

is the rotational distance defined by the target surface resolution $\Delta x$ and the surface orientation $\gamma_{i}$ with respect to the laser path [133] [134]. The target-based approach minimizes the total number of samples acquired so takes the least amount of time to complete. The cost of the target-based approach is that features smaller than the target resolution but still resolvable by the scanner may be missed. This approach is useful where time is critical but resolving features smaller than the target resolution is not important.

In the spot-based approach, the inter-sample distance is defined by the resolution of the laser spot if resolution is no larger than the target resolution. The spot-based inter-sample separation $\theta^{\text {spot }}$ is defined by

$$
\theta^{s p o t}=\min _{i=1}^{N}\left\{\theta_{i}^{r e s}-\theta_{i}^{e r r}\right\}
$$

where $\theta_{i}^{\text {res }}$ is the rotational resolution and $\theta_{i}^{e r r}$ is an error margin based on the rotational measurement uncertainty of the scanner. The rotational resolution $\theta_{i}^{\text {res }}$ is found using

$$
\theta_{i}^{\text {res }}=\tan ^{-1}\left(\frac{\frac{d_{\min }}{2} \cos \left(\gamma_{i}\right)}{\hat{R}_{i}+\frac{d_{\min }}{2} \sin \left(\gamma_{i}\right)}\right)
$$


which takes into account the effect of surface orientation $\gamma_{i}$ on the arc-length of the shortest portion of the long axis of the laser spot $d_{\min }$ as illustrated in Figure 3.8. The strength of the spot-based approach is that features smaller than the target surface resolution are resolvable where the size of the laser spot permits. The cost of this approach is a significant increase in total scanning time; however, as can be seen in Table I-3 of Appendix I, the total scanning time can still be significantly less than the time required to perform a complete high-density scan of the TFoV. This approach is useful where time is less of a factor than resolving as many surface features as possible.

The subscans are generated by defining the dimensions of the RoI of each subscan. As discussed in Section 3.2, measurements are removed from the start and end of each scan line. The region consisting of all measurements that are not discarded as being boundary measurements is the RoI. In Appendix I, the RoI is represented by a solid line while the actual scan region is represented by a dashed line. Given an inter-sample and inter-scan line separation of $\theta^{s e p}$, each subscan is defined as being $\left(K_{\text {samples }}-1\right) \theta^{\text {sep }} \times\left(K_{\text {lines }}-1\right) \theta^{\text {sep }}$ where $K_{\text {samples }}$ is the number of measurements per scan line within the RoI, and $K_{\text {lines }}$ is the number of scan lines within the RoI. If the target-based approach is used then $\theta^{\text {sep }}=\theta^{\text {target }}$ while if the spot-based approach is used then $\theta^{\text {sep }}=\theta^{\text {spot }}$.

The entire Rescan region is initially bounded by a box, the dimensions of which are integer multiples of the dimensions of a subscan RoI. The top graph on each page of Appendix I shows the rescan region bounding box. The solid line represents the region bounded by integer multiples of the subscan RoI region dimensions and is allowed to exceed the dimensions of the anchor scan. The dashed line represents the total area covered by all subscans if performed for the entire bounding box. Each neighbouring subscan RoI is allowed to overlap by a single row or column.

After the bounding box has been subdivided, all subscans that do not cover a portion of the Rescan region are discarded. Each subscan is then moved such that it maximizes the coverage of the Rescan region it previously covered while minimizing its coverage of 
the unscannable region. The process of shifting each subscan also reveals rescan regions that are redundantly covered by one or more subscans. These redundant subscans are eliminated to further reduce the number of subscans performed and, by extension, the number of measurements acquired is further reduced. The middle graph on each page of Appendix I shows the shifted subscan map for a target-based scan, while the graph at the bottom of each page shows the subscan map for the spot-based approach.

Table I-2 and Table I-3 show the number of subscans required to cover the Rescan region, total scanning and processing times for all anchor and subscans, the number of measurements obtained during the scanning process, and the time and sampling efficiencies of the subscan method compard to both a full and reduced scan. A full scan consists of sampling the TFoV with an inter-sample density based on the spot size at the beam waist. A reduced scan consists of scanning only the area covered by the bounding box with an inter-sample density based on the spot size at the beam waist. Table I-1 shows the predicted scan and processing time, as well as the number of measurments that would be obtained, using either a full scan or a reduced scan. In short, the full scan represents the worst case using contemporary methods, and the reduced scan represents the best case using contemporary methods.

It is clear from Table I-2 that using subscans with a target-based approach reduces the scan time to no more than $10.6 \%$ of the full scan time and requires no more than $9.0 \%$ of the measurements that would be required to complete a full scan. Compared to the more conservative reduced scan time estimate, target-based subscans generally require no more than $20.3 \%$ of the time and no more than $13.1 \%$ of the measurements required for a reduced scan. The positions of the subscans is generated automatically with minimal operator intervention whereas manually achieving the reduced scan results requires an $a$ priori examination of the surface to calculate the inter-sample separation. The greatest improvements in scanning time compared to either full or reduced scans were observed 
for surfaces with large planar regions (Figure I-2, Figure I-5, Figure I-11 and Figure I-14) or that feature an irregular silhouette (Figure I-2 and Figure I-11).

Subscans using a spot-based approach were observed to reduce the scan time to no more than $63.7 \%$ of the full scan time and the number of samples to no more than $60.1 \%$ of number of measurements in the full scan according to Table I-3; however, the smaller subscan regions are better targeted and cover the Rescan region more precisely. On the other hand, the scan time and sampling is only less than reduced scan time and sampling when large portions of the scene contain planar surfaces. As was the case with the targetbased approach, the smallest reductions in scan time, either full or reduced, were observed for complex (Figure I-15) and non-planar (Figure I-9) surfaces. In particular, Experiment \#3 results were worse for the region scanning approach when compared to the reduced scan time and sampling. This represents a limit to the effectiveness of the region-scanning approach; specifically, the spot-based region scanning approach is only more effective if the scanning region is smaller than the TFoV and contains planar surfaces. Due to the limited effectiveness of the spot-based approach, only the target-based approach is used in the next chapter.

\subsection{Summary}

Quality metrics can be used to significantly reduce the number of regions scanned at high resolution, resulting in a significant reduction in total time spent scanning the surface. The quality-weighted spot size is used in an iterative process to automatically find the locally optimal position based on spot size, resolvability, and surface orientation. Planarity, orientation, reflectivity and resolvability quality metrics are then used to automatically generate a list of regions within the total field of view that should be rescanned at high resolution. In the next chapter we examine how these and other quality metrics can be used to merge subscans into the composite range image. 


\section{Chapter 4}

\section{Building the Composite Scan}

In the previous chapter, a region map of a locally optimal anchor scan was used to generate a series of subscans. In this chapter, the results of performing these subscans is evaluated, then the subscans are merged with the anchor scan to generate a composite range image (CRI). Measurements that are rotationally close enough to have arisen from nearly the same surface location are merged using a quality-weighted variant of the traditional minimum-variance maximum-likelihood estimation approach. The range component of each merged measurement is used to generate a repeatability quality metric. This metric is combined with the maximum within-scan total quality of all merged measurements associated with the surface location to generate a within-composite total quality metric. The within-scan total quality is generated for each measurement in the anchor and subscans using the five quality metrics described in Chapter 3. Three new quality metrics are introduced to more completely describe the quality space of each measurement: aliasing, integration, and enclosed. 


\subsection{Introduction}

The quality of each measurement in the anchor scan and in the subscans can be represented by a single value that indicates the aggregate quality of the measurement within the quality space. In Chapter 3, five quality metrics were introduced: outlier (Section 3.5.3), orientation (Section 3.6.1), resolvability (Section 3.6.2), planarity (Section 3.7.1), and reflectivity (Section 3.7.2). These quality metrics were used to generate a region map that indicated which region was likely to generate measurements that would be discarded (Unscannable region), which region was likely to generate measurements that would be redundant (Complete region), and which region should be rescanned at a higher sampling density (Rescan region). In order to determine the total quality of each measurement, three additional quality metrics are required: aliasing, integration, and enclosed. Some of these eight quality metrics are critical, or exclusive, in that if they are unacceptable then the measurement must be discarded. Others augment the total quality of each measurement in that having one or more of these attributes be of acceptable quality adds value to the measurement. The exclusive and augmenting quality metrics are combined to generate the total quality metric.

The subscan bounds were selected such that, when combined, they would completely cover the Rescan region; however, for various regions some portions of the rescan region may not be covered. For example, data collection errors can generate subscans with unusable data, or measurement uncertainty may result in the actual measurement spatial positions not coinciding with the expected spatial position of each measurement. For this reason, the coverage of the subscans must be compared to the Rescan region to determine if any additional subscans need to be performed.

Once the Rescan region has been completely rescanned, all measurements obtained from the scanner viewpoint are combined into a range image that is a composite of the anchor and subscan measurements. Considerable measurement redundancy is expected 
and can be used to evaluate how repeatable each measurement is for a given surface location. Measurement redundancy can be reduced in two ways: by discarding planar subscan measurements within planar regions, and by merging measurements that arise from nearly the same surface location. Traditional approaches to measurement merger include the modified-Kalman minimum-variance estimation [82] [83] and quality-weighted merger [3]; however, the two approaches can be combined to generate a quality-weighted minimum-variance estimator. Using this approach, the spatial and reflectivity measurement estimates are weighted both by the covariance of all merged measurements as well as their within-scan total quality.

\subsection{Total Quality}

It is useful to be able to encapsulate the quality of each measurement as a single value to represent its overall, or total, quality. In the previous chapter, five metrics were used to represent the quality of a laser range scanner measurement. In this section, three new quality metrics are introduced to expand the quality space of each measurement. All quality metrics are then combined to generate a within-scan total quality metric that is applied to the anchor scan and all subscans prior to merging them to form the CRI.

\subsubsection{Aliasing Quality Metric}

In Section 3.6.2 it was stated that one issue relating to measurement resolution was whether a given range image achieved the target resolution $\Delta x$ such that aliasing was minimized; however, spatial uncertainty makes it difficult to know precisely the extents of the region covered by each beam footprint [49] [105]. Aliasing, within the context of this discussion, occurs when the sampling density is not sufficient to resolve surface features to at least $\Delta x$ and is discussed in greater detail in Section 2.6.1. Although smaller surface 
features may exist, their existence is ignored for purposes of creating a useful quality metric.

The aliasing quality metric $C_{i}^{\text {alias }}$ represents the likelihood that the measurements in a scan are sufficiently close together that features at the target surface resolution $\Delta x$ will be detected. It is found by

$$
C_{i}^{\text {alias }}=\left\{\begin{array}{cc}
0 & d_{i}^{\text {upper }} \leq d_{i}^{\text {far }} \\
1-\frac{d_{i}^{\text {far }}-d_{i}^{\text {lower }}}{2 d_{i}^{\text {err }}} & d_{i}^{\text {lower }}<d_{i}^{\text {far }}<d_{i}^{\text {upper }} \\
1 & d_{i}^{\text {far }} \leq d_{i}^{\text {lower }}
\end{array}\right.
$$

where $d_{i}^{f a r}$ is the distance to farthest 8-neighbour of $\hat{\mathbf{p}}_{i}, d_{i}^{\text {lower }}=\Delta x-2 d_{i}^{\text {err }}$, and $d_{i}^{\text {upper }}$ was defined in Section 3.6.2 to be $d_{i}^{\text {upper }}=\Delta x+2 d_{i}^{\text {err }}$ [133] [134]. The error term $d_{i}^{\text {err }}$ is obtained using (3.19) and is discussed in Section 3.6.2. Measurements previously marked as planar $\left(C_{i}^{\text {planar }}=1\right)$ always have $C_{i}^{\text {alias }}=1$ because planar surfaces lack spatial surface features. The aliasing metric is assigned $C_{i}^{\text {alias }}=0$ if any of the 8-neighbours of $\mathbf{p}_{i}$ are non-return or are outside the VoI.

Appendix $\mathbf{J}$ shows the aliasing quality map of all surfaces featured in Appendix B. All maps were generated from anchor scans so the inter-measurement distance is significantly larger than the spot radius. As a result, the only measurements with a non-zero aliasing metric are planar measurements. Appendix $\mathrm{K}$ shows the aliasing quality map of one representative subscan from each surface featured in Appendix B. The aliasing quality for most measurements in Figure K-3 is ideal.

\subsubsection{Integration Quality Metric}

As discussed in Section 2.3.3, speckle noise arises when speckle elements on the surface illuminated by the laser spot are large when compared to the wavelength of the laser light [68] [69], and is generally countered by integrating a single measurement over several 


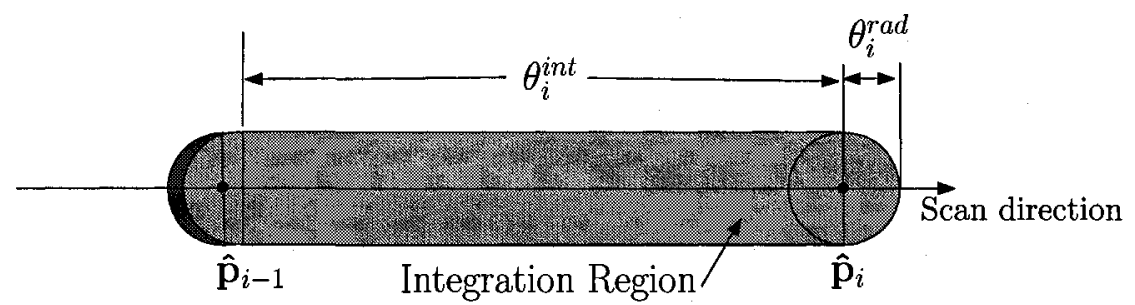

Figure 4.1: The range measurement uncertainty is reduced by integrating a measurement $\hat{\mathbf{p}}_{i}$ with instantaneous spot radius $\theta_{i}^{\text {rad }}$ over a distance $\theta_{i}^{\text {int }}$. The "Integration Region", shown in light grey, represents the region that contributes to the intensity profile, which is used to estimate the range and return signal intensity.

intensity samples as the laser spot is moved over the surface being scanned [70]. This is complicated by the need to minimize aliasing by ensuring that the measurements are separated by a distance less than the radius of the laser spot [52].

The integration quality metric represents the level of confidence in the accuracy of range measurements obtained using triangulation and continuous-wave laser range scanners, which can be affected by the distance the laser travels during the acquisition period as illustrated in Figure 4.1. It is obtained using

$$
C_{i}^{\text {int }}=\left\{\begin{array}{cc}
1 & \theta_{i}^{\text {int }} \leq \theta_{i}^{\text {rad }} \\
\frac{\theta_{i}^{\text {rad }}}{\theta_{i}^{\text {int }}} & \text { otherwise }
\end{array}\right.
$$

where $\theta_{i}^{i n t}$ is the rotational distance between measurement $\hat{\mathbf{p}}_{i}$ and the measurement $\hat{\mathbf{p}}_{i-1}$ that immediately preceded it, and $\theta_{i}^{r a d}$ is the radius of the laser spot in units of rotational distance [133] [134]. The integration metric is assigned $C_{i}^{i n t}=0$ if $\hat{\mathbf{p}}_{i-1}$ is not in the same scan line, or if either $\hat{\mathrm{p}}_{i}$ or $\hat{\mathrm{p}}_{i-1}$ is a non-return measurement. The integration quality metric represents a deviation from the expectation of an integration distance of no more than the spot radius so $C_{i}^{\text {int }}$ is an augmentative quality metric.

Appendix $\mathrm{J}$ shows the integration quality map of all surfaces featured in Appendix B. All maps were generated from anchor scans so the inter-measurement distance is 
significantly larger than the spot radius. As a result, all measurements were of low integration quality. Appendix K shows the integration quality map of one representative subscan from each surface featured in Appendix B. In Figure K-4, the integration quality is slightly lower for the more curved surfaces in which the inter-measurement surface distance is larger, as well as on the curved surfaces in Figure K-16 and Figure K-22. In Figure K-28, the change in integration quality is a result of the laser travelling a shorter distance between measurements where the planar surface is closer to the scanner. Moreover, the increased surface orientation with respect to the laser path results in a lengthening of the beam footprint, but not by as much as the inter-measurement surface distance increases. As a result, the integration quality of the measurements arising from this planar surface increases from upper left (closer to the scanner) to lower right (farther from the scanner).

\subsubsection{Enclosed Quality Metric}

Many of the quality metrics presented in this and the previous chapter are assigned $C_{i}^{\text {metric }}=0$ if any of the 8-neighbours of $\hat{\mathrm{p}}_{i}$ are non-return or are outside the VoI. In metrics such as the planarity quality metric (Section 3.7.1), the fit to a regression plane is most accurate when $\hat{\mathrm{p}}_{i}$ is completely enclosed within a neighbourhood in which all members of the neighbourhood are used to fit the plane. By extension, the orientation quality metric (Section 3.6.1) may also be questionable if based on less than a completely enclosed measurement because it is based on the normal of the regression plane. The resolvability (Section 3.6.2) and aliasing (Section 4.2.1) quality metrics are based on the surface distance between points which depends on a reasonably accurate estimate of the surface orientation.

The enclosed quality metric $C_{i}^{\text {enc }}$ indicates whether the quality metrics were obtained from a completely enclosed measurement or were estimated from neighbouring enclosed 
measurements. If a measurement is completely enclosed within an 8-neighbourhood then $C_{i}^{e n c}=1$ (enclosed); otherwise, $C_{i}^{e n c}=0$ (non-enclosed). Appendix J shows the enclosed quality map of all surfaces featured in Appendix B.

\subsubsection{Within-scan Total Quality Metric}

In Section 2.7, two types of total quality metrics were presented: weighted sum and binary product. Total quality could also be obtained using a quality product which has the form

$$
C_{i}^{\text {total }}=\prod_{j=1}^{N_{C}} C_{i, j}
$$

where $C_{i, j} \in[0,1]$ represents the $j^{\text {th }}$ quality metric associated with measurement $\hat{\mathbf{p}}_{i}$. In this case, $C_{i}^{\text {total }}=1$ only if $C_{i, j}=1 \forall j \in\left\{1, \ldots, N_{C}\right\}$, and $C_{i}^{\text {total }}=0$ if $\exists C_{i, j}=0$ for any $j \in\left\{1, \ldots, N_{C}\right\}$. Specifically, if any quality metric is zero (completely unacceptable) then the measurement has a total quality of zero.

One of the drawbacks of the quality product approach is that it does not permit any quality attribute to be unacceptable without considering the total quality of the measurement to be unacceptable. On the other hand, some quality attributes, such as surface orientation, resolvability, and reflectivity, may be critical indicators of total measurement quality. The solution is to use a hybrid approach in which some quality metrics are classified as critical or exclusive, while others are useful only in augmenting the per-measurement total quality. The total within-scan quality metric $C_{i}^{\text {total }}$ is the product of exclusive and augmenting quality metrics, and is obtained using

$$
C_{i}^{\text {total }}=C_{i}^{\text {exclusive }} \frac{1+C_{i}^{\text {augment }}}{2}
$$

where $C_{i}^{\text {exclusive }}$ was found using (3.26) and $C_{i}^{\text {augment }}$ is the augmenting quality average [133] [134]. The augmenting quality average $C_{i}^{\text {augment }}$ is found by

$$
C_{i}^{\text {augment }}=\frac{C_{i}^{\text {planar }}+C_{i}^{\text {enc }}+C_{i}^{\text {int }}+C_{i}^{\text {alias }}+C_{i}^{\text {out }}}{5} .
$$


It is important to note that a measurement is unacceptable only if $C_{i}^{\text {total }}=0$. Due to the cumulative nature of (4.4), a measurement can have $C_{i}^{\text {total }}$ close to zero, yet the measurement would still be of acceptable quality. The $C_{i}^{\text {total }}$ value of any measurement must be considered relative to other measurements obtained from the same surface region and not with respect to the ideal of $C_{i}^{\text {total }}=1$. Real measurements are often not ideal; they must merely be of acceptable quality to be useful in building a range model.

Appendix $\mathrm{J}$ shows the within-scan total quality map of all surfaces featured in Appendix B. The highest quality surfaces were the planar surfaces made of the same surface material used during the calibration process (Figure J-4, Figure J-19, and Figure J24); however, no surface displayed ideal quality because the inter-measurement distance is significantly larger than the spot radius. As a result, the integration quality of all measurements was low, resulting in a reduced augmenting quality. The bottom figure of each page shows the within-scan total quality in the form of an acceptance map: black represents measurements of acceptable quality, grey represents measurements of unacceptable quality, and white represents both non-return measurements and measurements from outside the VoI. The acceptability map more clearly illustrates which regions generate measurements that are suitable for use in the final model and which regions will need to be rescanned from another viewpoint.

Appendix $\mathrm{K}$ shows the within-scan total quality map of one representative subscan from each surface featured in Appendix B. In Figure K-11 the inner portions of the fissures are clearly discernable as lower-quality portions of the image. In Figure K-29 the portions of the picture (more clearly seen in Figure B-11) that are most similar in reflectivity to the calibration surface are clearly discernable as high-quality measurements against a lower-quality background. The bottom-right figure of each page shows the within-scan total quality of each subscan in the form of an acceptance map. 


\subsection{Region Coverage}

After the subscans have been acquired their coverage is examined to ensure that the Rescan region has been completely rescanned, and that the surface has been sampled to at least the target inter-sample surface distance $\Delta x$. Only the results of targetbased subscans are presented here; however, the methods can be applied to spot-based subscans as well. The region in which the target inter-sample surface distance has not been achieved, referred to as the Aliased region, is resampled at higher sampling density, then the region coverage is re-examined. The process continues until no Rescan or Aliased region remains.

\subsubsection{Rescan Region Coverage}

The surface coverage provided by the subscans must be compared to the Rescan region coverage to ensure that all portions of the Rescan region have been rescanned. The system used in this experiment generates scan patterns from the expected Cartesian centre of the scan, as well as the width of the scan pattern in Cartesian coordinates. As a result, there is sometimes a discrepancy between the expected scan coverage (based on spherical coordinates) and actual scan coverage (based on Cartesian coordinates) so that portions of the Rescan region are missed. As well, problems in the scan process can result in an unusable anchor or subscan that is automatically discarded.

As each subscan is processed, the region covered by the subscan is moved from the Rescan region to the Completed region. Once all subscans have been processed, the remaining portions of the Rescan region, if any, are then selected for second pass subscanning. Table L-1 shows the effect on the total scanning efficiency of performing secondary (Stage 2) scans. The number of Stage 1 scans refers to the Rescan region coverage discussed in Section 3.8, and the number of Stage 2 scans refers to the coverage of Aliased regions and regions not covered by Stage 1 subscans. Even after a second set of 
subscans, the fraction of full scan time is no more than $23.8 \%$ and the fraction of reduced scan time is no more than $47.7 \%$. The number of measurements obtained is no more than $37.4 \%$ and $65.9 \%$ of the number used for full and reduced scans respectively. The worst results were obtained, as would be expected, from surfaces like those in Experiment \#3 that contain few, if any, planar surfaces.

Appendix L shows the results of analyzing subscan coverage of the Rescan region. The upper left image on each page shows the actual coverage of each subscan as a black box. The upper right image shows the unscanned portions of the Rescan region as dark (blue) patches and the Completed region as light (green) patches. The Unscannable region is shown in white, while the light (red) patches are discussed in Section 4.3.3. In general, planar regions were best covered (Figure L-2, Figure L-5, and Figure L-14), and complex portions of the surface (Figure L-11) generated the most unscanned Rescan regions.

\subsubsection{Subscan Overlap}

Assuming all subscans were obtained without error, Rescan region coverage can be increased by increasing the degree of overlap among subscans. Moreover, increasing overlap increases the number of initial subscans slightly, but greatly reduces the number of second subscans required to provide complete Rescan and Aliased region coverage. If portions of the Rescan region remain after the initial set of subscans have been completed, then the degree of overlap is automatically increased for subscans used in this or future CRI generation.

The degree of non-coverage of the Rescan region is determined by first ensuring that all requested subscans have been completed. If any facets remain that have been marked for Rescan, then the number of elements in the overlap region is increased. Table 4.1 shows the results of increasing the degree of overlap on the number of initial and second subscans, as well as on the degree of initial scan coverage. For purposes of illustration, 
Table 4.1: Subscans coverage with increasing overlap.

\begin{tabular}{|c|cc|cc|cc|}
\hline $\begin{array}{c}\text { Overlap } \\
\text { Width }\end{array}$ & Relative & Relative & \# Initial & \# Second & Total \# & Total Scan \\
Sliasing & Coverage & Subscans & Subscans & Subscans & Time (min) \\
\hline \hline 0 & 0.0700 & 0.0214 & 16 & 43 & 59 & 10.39 \\
4 & 0.0253 & 0.0061 & 17 & 26 & 43 & 7.75 \\
7 & 0.0601 & 0.0030 & 17 & 39 & 56 & 10.04 \\
11 & 0.0217 & 0.0000 & 18 & 25 & 43 & 7.57 \\
\hline
\end{tabular}

the increase in overlap was applied to the same surface (Experiment \#3) within the same CRI. In practice, the degree of overlap is increased as needed during the course of scanning. The size of the overlap window was initially defined to be 0 elements and was increased after each set of subscans. As the degree of overlap was increased, the relative degree of coverage decreased until the entire Rescan region was covered (Relative Coverage $=0.0000$ ). The cost was a slight increase in the number of initial subscans, but the total scan time decreased. Table 4.2 shows that the scanning efficiency also increased with an increase in subscan overlap. In effect, the scanning process adapts to the degree of Rescan region coverage, resulting in increased scanning efficiency. The effect of increasing the overlap window size is graphically illustrated in Figure 4.2 to Figure 4.4.

Table 4.2: Subscan efficiency with overlap region.

\begin{tabular}{|c|ccc|}
\hline $\begin{array}{c}\text { Overlap } \\
\text { Width }\end{array}$ & $\begin{array}{c}\text { Fraction of } \\
\text { Full Scan }\end{array}$ & $\begin{array}{c}\text { Fraction of } \\
\text { Reduced Scan }\end{array}$ & $\begin{array}{c}\text { Fraction of } \\
\text { No Overlap }\end{array}$ \\
\hline \hline 0 & 0.09 & 0.28 & N/A \\
4 & 0.07 & 0.21 & 0.75 \\
7 & 0.08 & 0.27 & 0.97 \\
11 & 0.06 & 0.21 & 0.73 \\
\hline
\end{tabular}




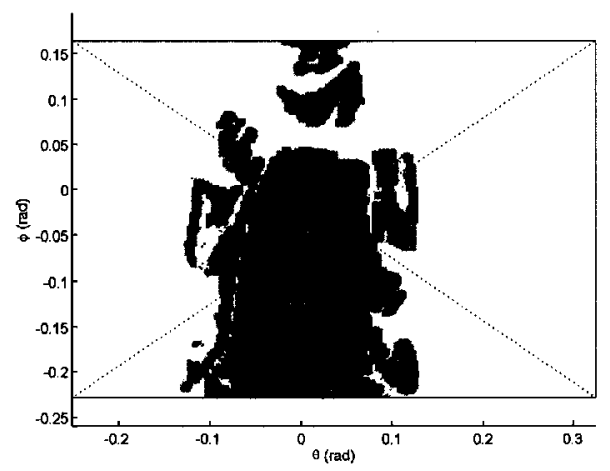

Figure 4.2: 4-element subscan overlap.

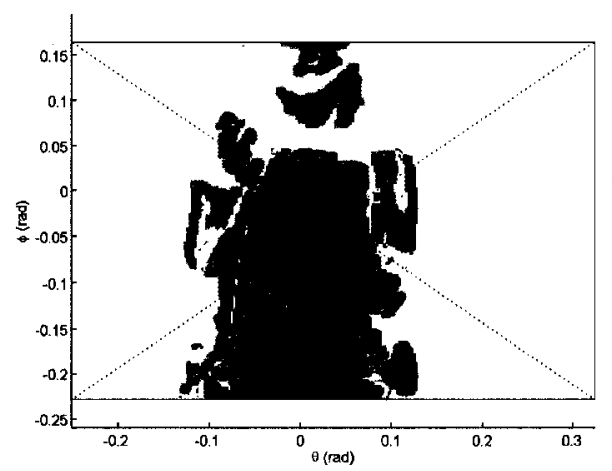

Figure 4.3: 7-element subscan overlap.

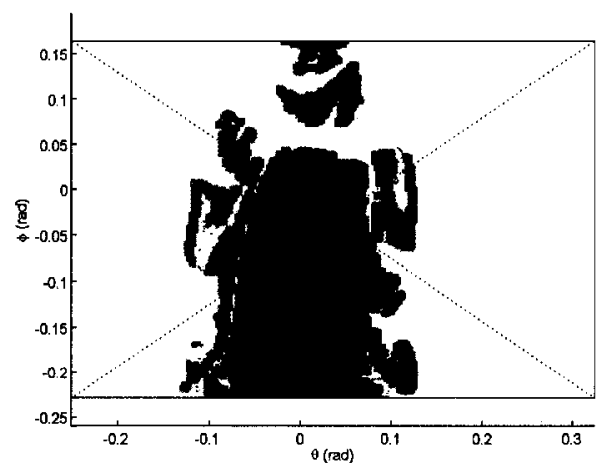

Figure 4.4: 11-element subscan overlap. 


\subsubsection{Anti-aliasing Coverage}

The goal of performing subscans is to sample the surface to at least some target intersample surface distance $\Delta x$ in regions that are of sufficiently low quality, measured using the aliasing metric introduced in Section 4.2.1, to warrant rescanning. Although the intermeasurement rotational distance of the subscans is selected to minimize the possibility of aliasing with respect to the target inter-measurement surface distance, aliasing can still occur. Even after taking measurement rotational uncertainty into account, aliasing can still occur so must be detected and compensated for by increasing the sampling density of each subscan.

Aliasing is detected by searching for all Enclosed $\left(C_{i}^{\text {enc }}=1\right)$, Resolvable $\left(C_{i}^{\text {resolve }}>0\right)$, Non-angled $\left(C_{i}^{\text {orient }}>0\right.$ ) subscan measurements with $C_{i}^{\text {alias }} \geq T^{\text {alias }}$ where $T^{\text {alias }}$ is the minimum acceptable aliasing quality. The region map facet in which this measurement occurs is then marked as Aliased. The aliasing quality threshold can be selected by the user to be conservative $\left(T^{\text {alias }}=0\right)$ or moderate $\left(T^{\text {alias }}=0.5\right)$ depending upon whether an error margin should (conservative) or should not (moderate) be included in the detection process. In this study a conservative setting was used to maximize aliasing detection.

All Aliased facets are added to the Rescan region after subscan coverage analysis has been performed. The inter-measurement rotational distance is then reduced by a predefined value $D_{\text {alias }}$ so that the sampling density is increased. The new sampling density is then applied to this surface. If Aliasing is still detected then the sampling density is once again increased. The $D_{\text {alias }}$ value that resulted in no Aliased facets being generated is then used for all future subscans. In this way, the scanning process adapts to the degree of aliasing such that the probability of aliasing being detected in future subscans is reduced.

Table 4.3 shows the results of decreasing the inter-sample distance on the degree of aliasing, as well as on the degree of Rescan region coverage. For purposes of illustration, 
Table 4.3: Subscans aliasing reduction with reduced inter-sample separation.

\begin{tabular}{|c|cc|cc|cc|}
\hline $\begin{array}{c}\text { Inter-sample } \\
\text { Separation }\end{array}$ & Relative & Relative & \# Initial & \# Second & Total \# & Total Scan \\
Subseans & Coverage & Subscans & Subscans & Subscame (min) \\
\hline \hline$\theta^{\text {sep }}$ & 0.0700 & 0.0214 & 16 & 24 & 40 & 7.05 \\
$0.75 \theta^{\text {sep }}$ & 0.0014 & 0.0465 & 24 & 30 & 54 & 9.51 \\
$0.56 \theta^{\text {sep }}$ & 0.0000 & 0.0472 & 39 & 38 & 77 & 13.56 \\
\hline
\end{tabular}

the process of adapting inter-sample rotational separation was applied only to Experiment \#3. The step value for inter-sample separation was defined to be $D_{\text {alias }}=0.75$. Relative aliasing (column 2) was reduced to zero after two iterations so future subscans of this object would be performed assuming $D_{\text {alias }}=0.75 * 0.75=0.5625$. This value would only be reduced if Aliased facets were detected. Table 4.4 shows the reduction in scanning efficiency as the subscan density was increased. Even after compensating for measurement aliasing, the scanning efficiency is still significantly higher than for either a full or a reduced scan.

Table 4.4: Subscan efficiency with reduced inter-sample separation.

\begin{tabular}{|c|cc|}
\hline $\begin{array}{c}\text { Inter-sample } \\
\text { Separation }\end{array}$ & $\begin{array}{c}\text { Fraction of } \\
\text { Full Scan }\end{array}$ & $\begin{array}{c}\text { Fraction of } \\
\text { Reduced Scan }\end{array}$ \\
\hline \hline$\theta^{\text {sep }}$ & 0.06 & 0.19 \\
$0.75 \theta^{\text {sep }}$ & 0.08 & 0.26 \\
$0.56 \theta^{\text {sep }}$ & 0.11 & 0.37 \\
\hline
\end{tabular}

\subsubsection{Combining Overlap and Anti-aliasing}

One side effect of increasing sampling density is that the degree of coverage of the Rescan region is reduced. Table 4.5 shows that applying the 11 -element overlap to the $0.56 \theta^{\text {sep }}$ 
inter-sample separation does not reduce the Relative Coverage to zero as it did when the inter-sample separation was $\theta^{\text {sep }}$ (see Table 4.1). Increasing the overlap region to 14 elements further reduces the Relative Coverage; however, increasing the overlap region to 18 elements results in a significant increase in the number of subscans required. The increase in the number of subscans using the 18 element overlap results in a greater scanning time (10.74 minutes) than would be required when the overlap is only 14 elements (10.04 minutes) so the overlap region is not increased beyond 14 elements. Table 4.6 shows that the scanning efficiency is maximized when using the 14-element window. This situation illustrates the importance of considering the total efficiency when deciding whether or not to increase the overlap region: the overlap region is not increased if it results in an increase in the total scanning time.

Table 4.5: Combining subscan overlap with $0.56 \theta^{\text {sep }}$ inter-sample rotational separation.

\begin{tabular}{|c|cc|cc|cc|}
\hline $\begin{array}{c}\text { Overlap } \\
\text { Width }\end{array}$ & Relative & Relative & \# Initial & \# Second & Total \# & Total Scan \\
Susing & Coverage & Subscans & Subscans & Subscans & Time (min) \\
\hline \hline 0 & 0.0000 & 0.0472 & 39 & 38 & 77 & 13.56 \\
11 & 0.0000 & 0.0066 & 53 & 10 & 63 & 11.10 \\
14 & 0.0000 & 0.0028 & 54 & 3 & 57 & 10.04 \\
18 & N/A & N/A & 61 & N/A & 61 & 10.74 \\
\hline
\end{tabular}

\subsection{Composite Range Image Generation}

The CRI is generated by merging measurements from each subscan with measurements obtained in the anchor scan. The CRI would later be combined with CRIs from other viewpoints to generate the surface model of the object being scanned; however, CRI merger is not addressed in the current study. A traditional approach to measurement 
Table 4.6: Efficiency of combining subscan overlap with $0.56 \theta^{\text {sep }}$ inter-sample rotational separation.

\begin{tabular}{|c|ccc|}
\hline $\begin{array}{c}\text { Overlap } \\
\text { Width }\end{array}$ & $\begin{array}{c}\text { Fraction of } \\
\text { Full Scan }\end{array}$ & $\begin{array}{c}\text { Fraction of } \\
\text { Reduced Scan }\end{array}$ & $\begin{array}{c}\text { Fraction of } \\
\text { No Overlap }\end{array}$ \\
\hline \hline 0 & 0.11 & 0.37 & N/A \\
11 & 0.09 & 0.30 & 0.82 \\
14 & 0.08 & 0.27 & 0.74 \\
18 & 0.09 & 0.29 & 0.79 \\
\hline
\end{tabular}

merger is the minimum-variance maximum likelihood [82] [83], or Kalman [141]-[146], estimator, that is sometimes referred as the modified Kalman minimum-variance (MKMV) estimator [82]. A less common approach is to use the spherical, rather than Cartesian, coordinate system to perform MKMV merging [147]-[150]; however, in the spherical covariance matrix the radial and rotational elements are decoupled. This is computationally simpler to perform so should reduce the effect of cumulative error.

The potential for measurement redundancy provides an opportunity to test the repeatability of the measurement process. Highly repeatable measurements can be trusted as being reasonably good estimates of the true surface spatial and reflectivity values, while measurements that are not repeatable are considered questionable because it is uncertain which measurements, if any, are representative of the true surface spatial and reflectivity values. The repeatability quality metric are then combined with the cumulative within-scan total quality to generate a within-composite total quality metric.

\subsubsection{Initialization Procedure}

Consider an anchor scan with $N_{\text {anchor }}$ measurements $\hat{\mathbf{p}}_{i, \text { Anchor }}$, represented in spherical coordinates. The associated model-based covariance of $\hat{\mathbf{p}}_{i, \text { Anchor }}$ is $S_{i, \text { Anchor }}$, and the 
measurement has a within-scan total quality of $C_{i, \text { Anchor }}^{\text {Total }}$. The CRI is initialized with all return measurements $\hat{\mathbf{p}}_{i, \text { Anchor }}$ that are within the VoI. Specifically,

- $\mathbf{p}_{i, C R I}=\hat{\mathbf{p}}_{i, \text { Anchor }}$ represents a spatial measurement in the CRI;

- $\Sigma_{i, C R I}=S_{i, A n c h o r}$ represents the covariance of the spatial measurement in the CRI;

- $I_{i, C R I}=\hat{I}_{i, A n c h o r}$ represents the return signal intensity of $\mathbf{p}_{i, C R I}$ in the CRI;

- $\sigma_{I_{i, C R I}}^{2}=s_{\hat{I}_{i, A n c h o r}}^{2}$ represents the variance of the return signal intensity;

- $C_{i, C R I}^{w s T o t a l}=C_{i, A n c h o r}^{T o t a l}$ is the cumulative within-scan total quality; and

- $C_{i, C R I}^{R e p e a t}=1$ is the repeatability quality metric.

\subsubsection{Update Procedure}

All measurements from the anchor scan and all subscans arise from the same scanner viewpoint, so measurements from nearly the same surface region are rotationally close. Specifically, two measurements $\hat{\mathbf{q}}_{i}=\left[\hat{\theta}_{i}, \hat{\phi}_{i}\right]^{T}$ and $\hat{\mathbf{q}}_{j}=\left[\hat{\theta}_{j}, \hat{\phi}_{j}\right]^{T}$ expressed in spherical coordinates, with rotational covariances

$$
Q_{i}=\left[\begin{array}{cc}
s_{\theta_{i}}^{2} & 0 \\
0 & s_{\phi_{i}}^{2}
\end{array}\right]
$$

and

$$
Q_{j}=\left[\begin{array}{cc}
s_{\theta_{j}}^{2} & 0 \\
0 & s_{\phi_{j}}^{2}
\end{array}\right]
$$

respectively are likely to be rotationally similar at $P<\alpha$ if

$$
d^{2}\left(\left\{\hat{\mathbf{q}}_{i}, Q_{i}\right\},\left\{\hat{\mathbf{q}}_{j}, Q_{j}\right\}\right) \leq \chi^{2}(2, \alpha)
$$


where $d^{2}\left(\left\{\hat{\mathbf{q}}_{i}, Q_{i}\right\},\left\{\hat{\mathbf{q}}_{j}, Q_{j}\right\}\right)$ is the Mahalanobis interpoint distance between $\hat{\mathbf{q}}_{i}$ and $\hat{\mathbf{q}}_{j}$ in the rotational plane. Note that the relationship between the Mahalanobis interpoint distance and the $\chi^{2}$ statistic is true only if the covariance matrices are diagonal. If $\alpha=0.05$ then $\chi^{2}(2, \alpha) \approx 5.99$ [138]. The Mahalanobis interpoint distance is found by

$$
d^{2}\left(\left\{\hat{\mathbf{q}}_{i}, Q_{i}\right\},\left\{\hat{\mathbf{q}}_{j}, Q_{j}\right\}\right)=\left(\hat{\mathbf{q}}_{i}-\hat{\mathbf{q}}_{j}\right)^{T}\left(Q_{i}+Q_{j}\right)^{-1}\left(\hat{\mathbf{q}}_{i}-\hat{\mathbf{q}}_{j}\right)
$$

where $\hat{\mathbf{q}}_{i}$ and $\hat{\mathbf{q}}_{j}$ arise from normally distributed populations [141] [146]. Generally $\alpha=$ $0.05[146]$ so $\chi^{2}(2, \alpha) \approx 5.99[138]$.

A $\chi^{2}$ test of the rotational Mahalanobis interpoint distance is used to determine if a measurement $\hat{\mathbf{p}}_{i, s u b s c a n}=\left[\hat{R}_{i, s u b s c a n} \hat{\mathbf{q}}_{i, \text { subscan }}^{T}\right]^{T}$ from a subscan corresponds to a measurement $\mathbf{p}_{i, C R I}=\left[R_{i, C R I} \mathbf{q}_{i, C R I}^{T}\right]^{T}$ in the CRI. Specifically, if

$$
d^{2}\left(\left\{\hat{\mathbf{q}}_{i, s u b s c a n}, Q_{i, s u b s c a n}\right\},\left\{\mathbf{q}_{i, C R I}, \Gamma_{i, C R I}\right\}\right) \leq \chi^{2}(2, \alpha)
$$

for $\alpha=0.05$ then $\hat{\mathbf{p}}_{i, s u b s c a n}$ is said to rotationally correspond to $\mathbf{p}_{i, C R I}$ where

$$
Q_{i, s u b}=\left[\begin{array}{cc}
s_{\theta_{i, \text { subscan }}}^{2} & 0 \\
0 & s_{\phi_{i, \text { subscan }}}^{2}
\end{array}\right]
$$

is the rotational covariance of $\hat{\mathbf{p}}_{i, s u b}$ and

$$
\Gamma_{i, s u b}=\left[\begin{array}{cc}
\sigma_{\theta_{i, C R I}}^{2} & 0 \\
0 & \sigma_{\phi_{i, C R I}}^{2}
\end{array}\right]
$$

is the rotational covariance of $\mathbf{p}_{i, C R I}$. Both $Q_{i, s u b}$ and $\Gamma_{i, s u b}$ are diagonal so (4.10) can be simplified to

$$
\begin{aligned}
d^{2}\left(\left\{\hat{\mathbf{q}}_{i, s u b s c a n}, Q_{i, s u b s c a n}\right\},\right. & \left.\left\{\mathbf{q}_{i, C R I}, \Gamma_{i, C R I}\right\}\right) \\
= & d^{2}\left(\left\{\hat{\theta}_{i, \text { subscan }}, s_{\hat{\theta}_{i, \text { subscan }}^{2}}^{2}\right\},\left\{\theta_{i, C R I}, \sigma_{\theta_{i, C R I}}^{2}\right\}\right) \\
& +d^{2}\left(\left\{\hat{\phi}_{i, s u b s c a n}, s_{\hat{\phi}_{i, s u b s c a n}}^{2}\right\},\left\{\phi_{i, C R I}, \sigma_{\phi_{i, C R I}}^{2}\right\}\right)
\end{aligned}
$$


where

$$
d^{2}\left(\left\{\hat{\theta}_{i, s u b s c a n}, s_{\hat{\theta}_{i, s u b s c a n}}^{2}\right\},\left\{\theta_{i, C R I}, \sigma_{\theta_{i, C R I}}^{2}\right\}\right)=\frac{\left(\hat{\theta}_{i, \text { subscan }}-\theta_{i, C R I}\right)^{2}}{s_{\hat{\theta}_{i, s u b s c a n}}^{2}+\sigma_{\theta_{i, C R I}}^{2}}
$$

and

$$
d^{2}\left(\left\{\hat{\phi}_{i, s u b s c a n}, s_{\hat{\phi}_{i, s u b s c a n}}^{2}\right\},\left\{\phi_{i, C R I}, \sigma_{\phi_{i, C R I}}^{2}\right\}\right)=\frac{\left(\hat{\phi}_{i, s u b s c a n}-\phi_{i, C R I}\right)^{2}}{s_{\hat{\phi}_{i, s u b s c a n}}^{2}+\sigma_{\phi_{i, C R I}}^{2}}
$$

are the Mahalanobis interpoint distances along each of the rotational axes.

If a subscan measurement $\hat{\mathbf{p}}_{i, s u b s c a n}$ has no rotationally corresponding measurement in the CRI then the subscan measurement is added to the CRI using the following procedure:

- $\mathbf{p}_{i, C R I}=\hat{\mathbf{p}}_{i, s u b s c a n}$

- $\Sigma_{i, C R I}=S_{i, \text { subscan }}$

- $I_{i, C R I}=\hat{I}_{i, \text { subscan }}$

- $\sigma_{I_{i, C R I}}^{2}=s_{\hat{I}_{i, s u b s c a n}}^{2}$

- $C_{i, C R I}^{w s \text { Total }}=C_{i, s u b s c a n}^{\text {Total }} ;$ and

- $C_{i, C R I}^{R e p e a t}=1$.

Otherwise, if $\hat{\mathbf{p}}_{i, \text { subscan }}$ rotationally corresponds to $\mathbf{p}_{i, C R I}$ then the update procedure proceeds as follows:

- If $C_{i, C R I}^{\text {wstotal }}>0$ and $C_{i, s u b s c a n}^{\text {total }}=0$ then no update is required (Option 1).

- If $C_{i, C R I}^{\text {wstotal }}=0$ and $C_{i, s u b s c a n}^{\text {total }}>0$ then the measurement is reinitialized (Option 2).

- If $C_{i, C R I}^{\text {wstotal }}=0$ and $C_{i, \text { subscan }}^{\text {total }}=0$ then the MKMV method [82] [83] (Option 3) is used. 
- If $C_{i, C R I}^{\text {wstotal }}>0$ and $C_{i, s u b s c a n}^{\text {total }}>0$ then the quality weighted MKMV method (Option 4) is used.

Each of the four update options are explained in the following sections. The repeatability metric update is discussed separately after $\mathbf{p}_{i, C R I}, \Sigma_{i, C R I}, I_{i, C R I}, \sigma_{I_{i, C R I}}^{2}$, and $C_{i, C R I}^{\text {wsTotal }}$ have been addressed because it is handled in the same way for all update options.

\section{Option 1: No update required}

The CRI consists of the highest-quality measurements from the anchor and subscans. If the current CRI measurement $\mathbf{p}_{i, C R I}$ has non-zero quality and the rotationally corresponding subscan measurement $\hat{\mathbf{p}}_{i, \text { subscan }}$ has a zero quality, then the $\hat{\mathbf{p}}_{i, \text { subscan }}$ is not of sufficient quality to be included in the CRI.

\section{Option 2: Reinitialization}

If the within-scan total quality of the subscan measurement $\hat{\mathbf{p}}_{i, s u b s c a n}$ is non-zero and the cumulative within-scan total quality of the rotationally corresponding measurement in the CRI $\mathbf{p}_{i, C R I}$ is zero then the measurement in the CRI is replaced with the subscan measurement. Specifically,

- $\mathbf{p}_{i, C R I}=\hat{\mathbf{p}}_{i, s u b s c a n}$

- $\Sigma_{i, C R I}=S_{i, s u b s c a n}$

- $I_{i, C R I}=\hat{I}_{i, s u b s c a n}$

- $\sigma_{I_{i, C R I}}^{2}=\sigma_{\hat{I}_{i, s u b s c a n}}^{2} ;$ and

- $C_{i, C R I}^{\text {wsTotal }}=C_{i, \text { subscan }}^{\text {Total }}$. 
In effect, any previous rotationally corresponding anchor and subscan measurements are discarded as being of insufficient quality. As indicated in Section 4.4.2, the CRI consists of the highest-quality measurements from the anchor and subscans. If the $\mathbf{p}_{i, C R I}$ is zero then all previously merged measurements must have had a quality of zero.

\section{Option 3: Standard MKMV}

If both $\mathbf{p}_{i, C R I}$ and $\hat{\mathbf{p}}_{i, s u b s c a n}$ are of zero quality then both are of insufficient quality to weight the measurements. In this case, the update procedure uses the standard MKMV estimator approach. The standard MKMV approach results in a spatial measurement estimate that is closer to the measurement with the smaller variance along each of the spatial axes. In this case, the axes are the spherical coordinate axes rather than the traditional Cartesian axes. As a result, the path between the measurements is curved in Cartesian space, but if the measurements are relatively close then the degree of curvature relative to the measurement uncertainty is small. The benefit of this approach is that the covariance matrices are diagonal so fewer calculations are required for each point pair.

The update procedure using the standard MKMV approach is summarized as follows:

- The spatial measurement estimate is updated using

$$
\mathbf{p}_{i, C R I}=W_{i}^{-1} \Sigma_{i, C R I}^{-1} \mathbf{p}_{i, C R I}+W_{i}^{-1} S_{i, s u b s c a n}^{-1} \hat{\mathbf{p}}_{i, s u b s c a n}
$$

where

$$
W_{i}=\Sigma_{i, C R I}^{-1}+S_{i, s u b s c a n}^{-1}
$$

is the weighting factor. This weights the estimated spatial measurement toward the measurement with the smaller variance through the inversion of the covariance matrices. This is the maximum-likelihood estimator of $\mathbf{p}_{i, C R I}$ and $\hat{\mathbf{p}}_{i, s u b s c a n}$ [151], as well as the Kalman estimate of the current state of the spatial measurement. 
- The covariance matrix estimate is updated using

$$
\Sigma_{i, C R I}=W_{i}^{-1}
$$

which is the covariance associated with the maximum-likelihood estimation of $\hat{\mathbf{p}}_{i, s u b s c a n}[151]$. It is also the posterior error estimate associated with the Kalman estimate of the current state of the spatial measurement.

- The return signal intensity estimate is updated using

$$
I_{i, C R I}=\frac{I_{i, C R I}}{w_{i} \sigma_{I_{i, C R I}}^{2}}+\frac{\hat{I}_{i, \text { subscan }}}{w_{i} s_{\hat{I}_{i, s u b s c a n}}^{2}}
$$

where

$$
w_{i}=\frac{1}{\sigma_{I_{i, C R I}}^{2}}+\frac{1}{s_{\hat{I}_{i, s u b s c a n}}^{2}}
$$

is the weighting factor. This weights the estimated return signal intensity toward the measurement with the smaller return signal intensity variance.

- The estimate of the return signal intensity variance is updated using

$$
\sigma_{I_{i, C R I}}^{2}=\frac{1}{w_{i}}
$$

- The cumulative within-scan total $C_{i, C R I}^{w s T o t a l}$ requires no updating because $C_{i, C R I}^{w s T o t a l}=$ $C_{i, s u b s c a n}^{\text {Total }}=0$.

\subsubsection{Option 4: Quality-weighted MKMV}

If both $\mathbf{p}_{i, C R I}$ and $\hat{\mathbf{p}}_{i, s u b s c a n}$ are of non-zero quality then in order for the CRI to consist of the highest quality measurements the total quality of each measurement must be included in the update equation. The update procedure is, in this case, as follows: 
- The spatial measurement estimate is updated using

$$
\mathbf{p}_{i, C R I}=W_{i}^{-1} C_{i, C R I}^{\text {wsTotal }} \Sigma_{i, C R I}^{-1} \mathbf{p}_{i, C R I}+W_{i}^{-1} C_{i, s u b s c a n}^{\text {Total }} S_{i, s u b s c a n}^{-1} \hat{\mathrm{p}}_{i, s u b s c a n}
$$

where

$$
W_{i}=C_{i, C R I}^{\text {wsTotal }} \Sigma_{i, C R I}^{-1}+C_{i, s u b s c a n}^{\text {Total }} S_{i, s u b s c a n}^{-1}
$$

is the weighting factor [134]. Note that $C_{i, C R I}^{w s T o t a l}$ and $C_{i, s u b s c a n}^{\text {Total }}$ are scalar quantities so weight each element in the covariance matrices. This not only weights the estimated spatial measurement toward the measurement with the smaller variance through the inversion of the covariance matrices, but weights the estimated spatial measurement toward the higher quality measurement.

- The covariance matrix estimate is updated using

$$
\Sigma_{i, C R I}=\frac{1}{C_{i, C R I}^{w s T o t a l}+C_{i, \text { subscan }}^{\text {Total }}} W_{i}^{-1}
$$

which weights the covariance matrix elements toward the higher-quality measurement $[134]$.

- The estimate of the return signal intensity is updated using

$$
I_{i, C R I}=\frac{C_{i, C R I}^{w s T o t a l}}{w_{i} \sigma_{I_{i, C R I}}^{2}} I_{i, C R I}+\frac{C_{i, \text { subscan }}^{\text {Total }}}{w_{i} s_{\hat{I}_{i, s u b s c a n}}^{2}} \hat{I}_{i, s u b s c a n}
$$

where

$$
w_{i}=\frac{C_{i, C R I}^{w s T o t a l}}{\sigma_{I_{i, C R I}}^{2}}+\frac{C_{i, \text { subscan }}^{\text {Total }}}{s_{\hat{I}_{i, s u b s c a n}}^{2}}
$$

is the weighting factor. 
- The return signal intensity variance estimate is updated using

$$
\sigma_{i, C R I}^{2}=\frac{1}{w_{i} C_{i, C R I}^{w s T o t a l}+w_{i} C_{i, \text { subscan }}^{\text {Total }}} .
$$

- The cumulative within-scan total is updated using the quality-weighted scalar equivalent variance of the quality-weighted MKMV estimator approach. The scalar equivalent variance represents the variance along the line between two measurements and is explained in Appendix M. All measurements are represented in spherical coordinates so, in this case, the line is the geometric line between two spherical measurements. The update equation is

$$
C_{i, C R I}^{w s T o t a l}=m_{i, C R I} C_{i, C R I}^{w s T o t a l}+m_{i, s u b s c a n} C_{i, s u b s c a n}^{T o t a l}
$$

where

$$
m_{i, C R I}=\frac{C_{i, C R I}^{w s T o t a l}}{m_{i} \sigma\left(\hat{\mathbf{p}}_{i, s u b s c a n} \mid \mathbf{p}_{i, C R I}, \Sigma_{i, C R I}\right)^{2}}
$$

and

$$
m_{i, \text { subscan }}=\frac{C_{i, \text { subscan }}^{\text {Total }}}{m_{i} \sigma\left(\mathbf{p}_{i, C R I} \mid \hat{\mathbf{p}}_{i, C R I}, S_{i, \text { subscan }}\right)^{2}}
$$

are the weighting factors. This time, the total weight $m_{i}$ is found using

$$
m_{i}=\frac{C_{i, C R I}^{\text {wsTotal }}}{\sigma\left(\hat{\mathbf{p}}_{i, \text { subscan }} \mid \mathbf{p}_{i, C R I}, \Sigma_{i, C R I}\right)^{2}}+\frac{C_{i, \text { subscan }}^{\text {Total }}}{\sigma\left(\mathbf{p}_{i, C R I} \mid \hat{\mathbf{p}}_{i, C R I}, S_{i, \text { subscan }}\right)^{2}}
$$

so that the unit-variance terms are weighted by the total quality of each measurement [134].

\subsubsection{Non-return Measurements}

Non-return measurements in subscans are included in the merge process and represent a special case. Consider that anchor scan measurements obtained using triangulation or 
continuous-wave laser range scanners are cumulative over the scan path. As a result, the spatial location of the measurement may not refer to a real surface but an estimate based on the integration over the spatial acquisition interval. Subscan measurements are generated using much smaller spatial integration intervals so it is possible to generate non-return subscan measurements that rotationally correspond to return anchor scan measurements.

If a subscan measurement $\hat{\mathbf{p}}_{i, \text { subscan }}$ rotationally corresponds to an anchor measurement $\mathbf{p}_{i, C R I}$ that has not been merged with any previous subscan measurements then:

- The measurement estimate $\mathbf{p}_{i, C R I}$ and its associated covariance are removed from the CRI;

- The return signal intensity measurement $I_{i, C R I}$ and its associated variance are removed from the $\mathrm{CRI}$; and

- The quality metrics associated with $\mathbf{p}_{i, C R I}$ are removed from the CRI.

This process results in the silhouette of the object being refined using measurements obtained from denser scan patterns. Non-return subscan measurements are then discarded to minimize the number of measurements in the CRI.

\subsubsection{Repeatability Quality Metric}

The repeatability quality metric $C_{i, C R I}^{\text {repeat }}$ represents the change in the range measurement resulting from merging two measurements. A highly repeatable measurement displays little change when merged with nearby measurements and indicates that the range measurement can be reliably obtained. As each subscan is merged into the CRI, the metric is updated for each measurement in which a subscan measurement is merged with a rotationally corresponding measurement in the CRI. As a result, $C_{i, C R I}^{\text {repeat }}$ may be 
recalculated multiple times for a single measurement, changing to represent the shift in range measurement after each merger.

The repeatability quality metric is updated using

$$
C_{i, C R I}^{r e p e a t}=\left\{\begin{array}{cc}
C_{i, C R I}^{r e p e a t} & \Delta R_{i, C R I} \leq R_{i, C R I}^{\text {err }} \\
\min \left\{C_{i, C R I}^{r e p e a t},\left(\frac{R_{i, C R I}^{e r r}}{\Delta R_{i, C R I}}\right)\right\} & \text { otherwise }
\end{array}\right.
$$

where $R_{i, C R I}^{e r r}=Z(\alpha / 2) \sigma_{R}$ is the two-tailed range error margin based on the modelbased range measurement uncertainty $\sigma_{R}$. Typically $\alpha=0.05$ is used so $Z(\alpha / 2)=$ 1.96 [135]. The range measurement change between pre- and post-merger is represented by $\Delta R_{i, C R I}=\left|R_{i, C R I}^{\text {before }}-R_{i, C R I}^{a f t e r}\right|$ where the range measurement prior to merging the two measurements is $R_{i, C R I}^{b e f o r e}$ and the range measurement after merging the two measurements is $R_{i, C R I}^{a f t e r}$. In effect, $C_{i, C R I}^{\text {repeat }}$ is unchanged if the shift in range value is less than would be expected based on the uncertainty in the measurement.

\subsubsection{Results}

Appendix $\mathrm{N}$ shows the results of merging a cluster of subscans with measurements from the anchor scan into a CRI. In all cases, only four subscans are considered so that the effects of merging measurements can be more clearly illustrated. Subscans were selected to illustrate overlap among subscans in spatially complex regions where possible. The upper left graphic shows the within-scan total quality speckle plot with the subscans indicated as boxes. The upper-right image is a speckle plot of the within-scan total quality of all anchor and subscan measurements superimposed prior to merger.

The lower-left images show the repeatability quality metric as a speckle plot. Lowrepeatability measurements are light grey while completely repeatable measurements are represented in black. The repeatability metric has a range $(0,1]$ so $C_{i, C R I}^{\text {repeat }}$ is always greater than zero. The lower-right images show the within-scan total quality metric after 
measurement merger. Subscans that were merged with anchor scan measurements appear as light grey speckling of the within-scan total quality map.

\subsection{Within-composite Quality Metrics}

Quality metrics can be applied to the composite range image much like they were to each of the scan range images. In the previous section, two quality metrics were introduced: repeatability quality and within-scan total quality. This section introduces four withincomposite quality metrics: reflectivity, orientation, aliasing, and within-composite total. These metrics are analogous to reflectivity, orientation, aliasing and within-scan total quality metrics generated for each scan. The experimental results for each metric can be found in Appendix $\mathrm{O}$.

\subsubsection{Delaunay Neighbourhood}

Within-scan quality metrics were generated using raster scans so it was convenient to use the 8-neighbourhood of each measurement. As a result, within-scan quality metrics could be generated relatively quickly, often using vector mathematics. CRI measurements are not distributed in a regular pattern so it is not feasible to use 8-neighbourhoods unless the CRI were resampled as a grid-based surface. An alternative is to use the Delaunay triangulation of the CRI-based surface to generate a Delaunay neighbourhood of each measurement. The Delaunay neighbourhood of $\mathbf{p}_{i}$ is defined as the set $F_{i}$ of all $N_{i}$ measurements that share a Delaunay edge with $\mathbf{p}_{i}$. That is, $\mathbf{p}_{i}$ and $\mathbf{p}_{j}$ are Delaunay neighbours if $\exists E\left(\mathbf{p}_{i}, \mathbf{p}_{j}\right)$ where $E\left(\mathbf{p}_{i}, \mathbf{p}_{j}\right)$ is a Delaunay edge.

Delaunay neighbourhoods are a subset of 8-neighbourhoods: all members of a Delaunay neighbourhood in a raster scan will be 8-neighbours, but all 8-neighbours are not necessarily Delaunay neighbours. Figure 4.5 shows the Delaunay and 8-neighbourhoods of 

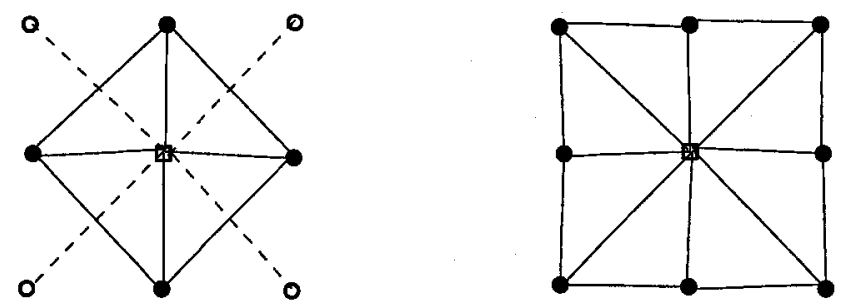

Figure 4.5: The filled circles represent Delaunay neighbours of a measurement (empty square). The filled and empty circles represent the 8-neighbours of a measurement. The Delaunay neighbourhood on the right is equivalent to the 8-neighbourhood, while the Delaunay neighbourhood on the left is smaller than the 8-neighbourhood.

a measurement. The illustration on the right shows the largest Delaunay-neighbourhood that can be obtained in a raster image and also represents an 8-neighbourhood of the same measurement. The illustration on the left shows the smallest Delaunay neighbourhood (filled circles) of a measurement. The 8 neighbourhood consists of all measurements marked with either empty of filled circles.

\subsubsection{Composite Smoothing}

Prior to generating the within-composite quality metrics, the CRI is smoothed using a median filter applied to each Delaunay neighbourhood. Specifically, the median filter size is defined to be that of the neighbourhood. The median filter replaces the range and intensity measurement with the median range and intensity measurement of all Delaunay neighbours of the measurement. An outlier quality metric was not generated because both the pre- and post-smoothed range and intensity values are best-guess estimates of the surface. The outlier metric represents the degree of deviation from the original measured values; however, measurement merger and post-acquisition smoothing result in the CRI measurements being estimates rather than measured values. 


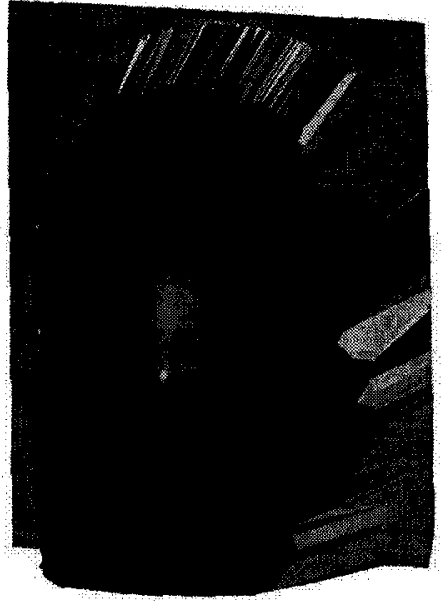

Figure 4.6: Experiment \#1 facet map.

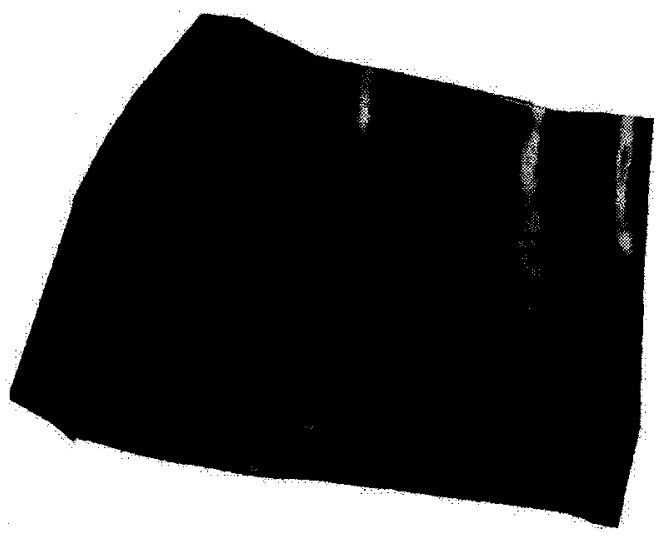

Figure 4.8: Experiment \#3 facet map.

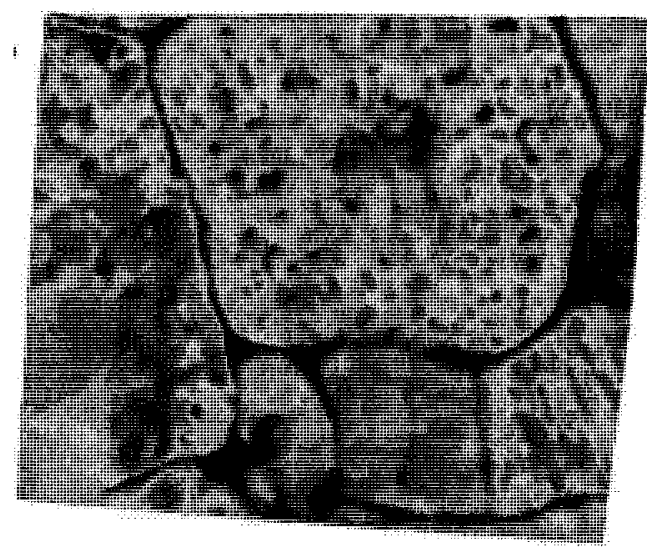

Figure 4.7: Experiment \#2 facet map.

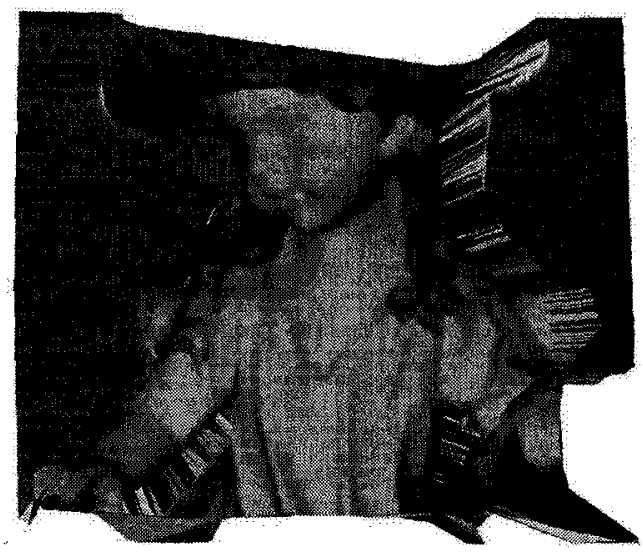

Figure 4.9: Experiment \#4 facet map.

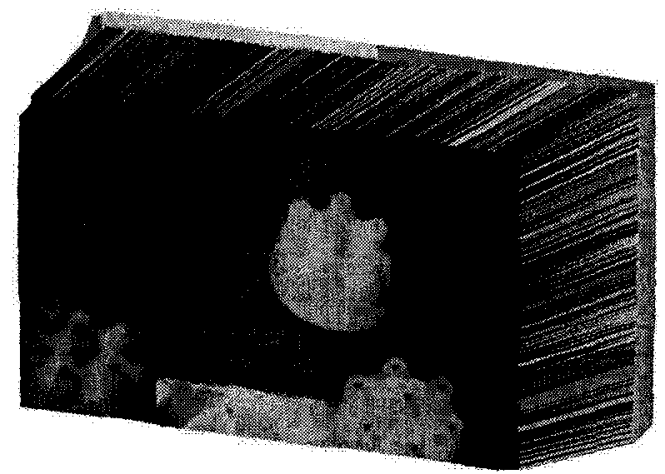

Figure 4.10: Experiment \#5 facet map. 
After the CRI has been smoothed, the return signal intensity estimates are converted into model-based reflectance values using (3.24). Figure 4.6 to Figure 4.10 show the final Delaunay surface map after smoothing. Facet colour is based on the estimated reflectance of the surface at each measurement.

\subsubsection{Within-composite Reflectivity Quality Metric}

The reflectance values for each measurement are used to generate the within-composite reflectivity metric using $C_{i, C R I}^{\text {reflect }}(3.25)$. The top-left images in Appendix O show the reflectivity quality map as a speckle plot for all experimental surfaces. In all experiments except Experiment \#5 the reflectivity quality showed no indication of being generated by merging subscans. In Experiment \#5 (Figure O-17), illumination changes during the scanning process changed the return signal intensity, resulting in a noticeable difference in reflectivity from the neighbouring scans. Future work will examine how to automatically detect and correct for illumination changes amoung neighbouring subscans.

\subsubsection{Within-composite Orientation Quality Metric}

The orientation of the surface through each measurement is approximated by finding the orientation of a plane fitted to each Delaunay neighbourhood. The surface orientation deflection from the line of sight to the scanner is then used to obtain a within-composite orientation metric $C_{i, C R I}^{\text {orient }}$ for each measurement, using (3.15). The orientation quality map for each surface can be seen as the upper-right image on each page of Appendix O. In Experiment \#2 (Figure O-6), the surface orientation increases toward the top of the image because the laser beam strikes the surface at a higher angle at higher rotation angles. A similar situation occurs in Experiment \#5 (Figure O-18) toward the bottom of the image. 


\subsubsection{Within-composite Aliasing Quality Metric}

In Section 4.2.1, the within-scan aliasing quality metric was introduced to detect whether the inter-measurement distance was sufficient to resolve surface features to at least $\Delta x$. Once again, although smaller surface features may exist, their existence is ignored for purposes of creating a useful quality metric. The within-composite aliasing metric $C_{i, C R I}^{\text {alias }}$ is obtained using (4.1), but this time $d_{i}^{f a r}$ is the distance to farthest Delaunay neighbour of $\mathrm{p}_{i}$ and $d_{i}^{\text {err }}$ is based on the covariance of each measurement after merger rather than the model-based uncertainty. Measurements with $C_{i, C R I}^{\text {orient }}=0$ are automatically assigned $C_{i, C R I}^{a l i a s}=0$ because the surface orientation is unacceptably steep. The bottom-left image on each page of Appendix $O$ represents the aliasing quality map as a speckle plot for each of the experimental surfaces.

\subsubsection{Within-composite Total Quality Metric}

As was the case with the anchor scan and subscans, the quality of each measurement can be represented by a single value to represent the cumulative quality of the measurement. The within-composite total quality metric $C_{i, C R I}^{t o t a l}$ is found using

$$
C_{i, C R I}^{\text {total }}=\frac{C_{i, C R I}^{w s T o t a l} C_{i, C R I}^{\text {orient }} C_{i, C R I}^{r e f l e c t}\left(2+C_{i, C R I}^{\text {repeat }}+C_{i, C R I}^{\text {alias }}\right)}{4}
$$

If any within-composite quality metric, except aliasing, is zero then the within-composite total quality is also zero. The within-composite total quality map for each experimental surface can be seen in the bottom-right of each page of Appendix $O$.

\subsection{Summary}

Two new quality metrics are introduced: aliasing and integration quality metrics, then are combined into a with-scan total quality metric. The aliasing metric is used to determine 
whether the subscan density was sufficient to avoid aliasing with respect to the target surface sampling resolution after taking measurement uncertainty into account. The integration metric is applicable only to continuous-wave and triangulation laser range scanners in which which each measurement is cumulative over a long surface region, refered to as the integration region. All quality metrics are classified as either exclusive or augmenting for purposes of combining them into a total quality metric. Quality metrics are exclusive if they must be non-zero for the measurement to be included in the final model. A quality metric is augmenting if it can be unacceptable (0) yet still be included in the final model.

Three of the quality metrics introduced in this and the previous chapter for use with raster scans can be adapted for non-grid data sets such as arise from merging multiple scans. Measurements that share a Delaunay edge with a measurement are defined as Delaunay neighbours of the measurement. Orientation and aliasing metrics are computed using the neighbourhood of a measurement so can be computed for either 8neighbourhoods, in the case of raster data, or for the more general Delaunay neighbours. A repeatability quality metric can also be defined to represent the quality of points in a composite range image based on the consistency in range measurement values. Specifically, if the range measurements of rotationally similar measurements do not vary by more than would be expected from model-based range uncertainty then the repeatability of the measurement is ideal.

The aliasing quality metric can be used to determine whether the scan density is sufficient to minimize the chance of aliasing with respect to the target surface sampling resolution. If aliased measurements are detected then the subscan density is automatically increased to avoid aliasing in future subscans. Experiments indicate that aliasing can be corrected for within a few iterations, and that the total scan time using automatic adaptation to aliasing is still significantly less than scanning the total field of view using a high-density scan. 
The degree of non-coverage of the rescan region can be used to automatically adjust the degree of overlap among subscans. Experiments indicate that a degree of overlap can be obtained within relatively few iterations that eliminates rescan region non-coverage, and that the total scanning time using the new degree of overlap is still less than for a single high-density scan. This result holds even when automatic anti-aliasing and automatic non-coverage correction are combined.

Rotationally similar measurements that contain quality information can be merged such that both the quality of each measurement and their covariance are combined to determine the spatial location and reflectivity of each point in the composite range image. The quality-weighted MKMV merge approach uses a variation of the maximum-likelihood estimator approach; the spatial and intensity values are weighted toward both the smaller covariance and the higher total quality. If only one of the rotationally similar pair of measurements is of non-zero total quality then the measurement without non-zero total quality is discarded. 


\section{Chapter 5}

\section{Conclusions and Future Work}

\subsection{Summary and Conclusions}

This dissertation is concerned with developing a method for adapting scan patterns to reduce the time required to obtain a $3 \mathrm{D}$ model, reduce dependence on operator experience, and to better represent the quality of the measurements obtained during the scanning process. In this study, the scanner viewpoint was assumed static; however, future research can extend the method to include multiple viewpoints. Tthe quality metrics can also be adapted for use with view planning procedures.

\subsubsection{Contributions and Innovations}

This dissertation presents the following innovations based on a combination of theoretical work and experimental evidence:

1. New quality metrics were derived to quantify:

- the deviation of model data from acquired data;

- the ability to resolve a surface to at least the target resolution; and 
- how well the surface around a measurement fits a plane.

2. Contemporary quality metrics were improved, including:

- surface orientation; and

- surface reflectivity.

3. A method for finding the locally optimal scanner viewpoint based on measurement quality was presented and demonstrated experimentally;

4. A method for finding the dimensions of a laser spot in the mid-field was derived;

5. A method was presented and demonstrated experimentally to automatically isolate which region is likely to generate useful measurements, which region is likely to generate measurements that will be discarded, and which region is likely to generate measurements that will add little to the model based on measurement quality;

6. An improved quality metric was derived to quantify whether the measurement density was sufficient to resolve the surface to at least the target resolution, taking into account both measurement resolution and measurement uncertainty;

7. A new quality metric was introduced to quantify the effect of how far a laser spot travels relative to the size of the spot during measurement acquisition;

8. A method was presented for merging spatial and intensity measurements based on both measurement uncertainty and measurement quality; and

9. A new quality metric was presented to quantify the reproducibility of range measurements obtained from nearly the same surface region.

This dissertation also presents the following contributions: 
1. Environmental factors affecting the quality of laser range scanner measurements were identified in contemporary literature;

2. A comprehensive survey was conducted of quality metrics for laser range scanners to identify areas where measurement quality has not been adequately addressed;

3. The relationship between laser spot area per unit measurement coverage and both filter window size and the number of times the filter is applied was examined experimentally, and the optimal filter window size and number of repetitions was determined experimentally;

4. A method was presented for automatically generating a sequence of subscans to perform high-density scanning of the region which is likely to generate useful measurements, with experimental evidence demonstrating that this approach was able to significantly reduce both the number of measurements acquired and the total scan time.

5. A method was presented and experimentally demonstrated for automatically adapting the degree of overlap among subscans to compensate for region non-coverage; and

6. A method was presented and experimentally demonstrated for automatically adapting the inter-sample distance to compensate for measurement aliasing with respect to the target sampling resolution.

\subsubsection{Quality Metrics}

In Chapter 2, a comprehensive survey of quality metrics for laser range scanners was presented in which the environmental factors affecting the quality of laser range scanner measurements were identified in contemporary literature, and areas where measurement 
quality has not been adequately addressed were identified. In general, most quality metrics have been designed for specific applications or specific algorithms, and are often used independently. Measurement uncertainty has been used extensively to represent measurement quality but many environmental factors affect measurement uncertainty, making it insufficient as an independent quality metric. For this reason, much of this study was concerned with the development of quality metrics that quantified those environmental factors that cause true measurement uncertainty to deviate from the standard uncertainty model.

The relationship of range and resolution to measurement quality was not well defined for mid-field measurements so this study focused on the shape of the beam in the midfield. In particular, the lengths of the long and short axes of the beam footprint were derived for an arbitrary surface orientation under the assumption that the surface within the beam could be approximated as a planar surface. The axial lengths of the beam footprint were then related to measurement quality through the resolvability quality metric. Moreover, the resolvability metric took into account the rotational uncertainty associated with each measurement such that the resulting quality metric represented the degree of likelihood that the desired measurement resolution could be achieved.

Sampling density, and, by extension, measurement aliasing, was identified as an environmental factor that has been represented using quality metrics, but most approaches were found to be highly application specific. An aliasing quality metric was designed to better represent whether the surface has been sampled to at least the desired sampling density. In particular, assuming each measurement is surrounded by valid (return, withinVoI) measurements, if the most distant neighbour is sufficiently close that aliasing is unlikely then aliasing is unlikely in any direction. Aliasing, in this context, is considered unlikely if the distance between samples is less than half the target surface resolution.

The region-generating approach presented in this dissertation was applied to a point scanner so the rotational components of the laser range scanner measurements were used 
to define the field of view. The approach can be applied to linear track scanners by defining the field of view as a combination of rotational and translational values, or to planar track scanners by defining the field of view using two translational parameters. In general, the field of view is defined by the range of motion through which the laser can be moved. In should, however, be noted that the scanner must be capable of multi-density scanning and of subscanning portions of the field of view for it to be possible to perform rescanning. This limits the number of systems capable of using the approach presented in this dissertation; however, it may be possible to work with suppliers to allow new, or even current, systems to perform multi-density and subregion scanning.

Contemporary quality metrics were found to perform adequately in assessing the quality of measurements within the context of specific applications, but were often not readily generalizable. Quality metrics were standardized such that the maximum quality (1) indicated a measurement of ideal quality, and the minimum quality (0) indicated that the measurement was of unacceptable quality. Quality metrics were then classified as either exclusive or augmenting for purposes of combining them into a total quality metric. Quality metrics are exclusive if they must be non-zero for the measurement to be included in the final model. A quality metric is augmenting if it can be unacceptable (0) yet still be included in the final model.

Few researchers had combined quality metrics so that the strengths of one may offset the weakness of the other. In this study, the information imparted by each quality metric was used to address a different issue within the context of minimizing the total number of measurements obtained while maximizing the quality of those measurements. Resolvability and orientation quality metrics were used to find the scanner viewpoint that maximized the number of measurements obtained with non-zero resolvability and orientation, and to identify measurements that would normally be discarded during postprocessing. The planarity quality metric was used to identify regions unlikely to contain surface spatial complexity, while outlier and reflectivity metrics were used, together with 
non-return measurements, to identify regions that should be rescanned. The aliasing metric was used to determine whether the subscan density was sufficient to avoid aliasing after considering measurement uncertainty. The integration metric was used to distinguish between anchor scan measurements, in which each measurement is cumulative over a large surface region, and subscan measurements, in which each measurement is cumulative over a small surface region.

\subsubsection{Adaptive Scanning}

Chapter 3 presented a method for finding the locally optimal scanner viewpoint, as well as for automatically scanning regions of interest while minimizing the total number of measurements acquired as well as the total scan time. This method was demonstrated using five different test surfaces including a wall segment (Experiment \#2), a complex and angled surface (Experiment \#5), a spatially simple surface with complex reflectivity (Experiment \#5), smoothly curved and glossy surface (Experiments \#1 and \#3), and a mix of planar and spatially complex surfaces (Experiments \#1, \#4, and \#5). Using these surfaces, it was demonstrated experimentally that quality metrics could be used to significantly reduce the number of regions scanned at high resolution, resulting in a significant reduction in total time spent scanning the surface.

Data smoothing was identified as a post-processing step that requires an experienced operator; however, the relationship between smoothing and scan density is generally ignored because contemporary approaches to scanning involve raster patterns of globally constant sampling density. Where sampling density changes, an expert operator is generally on hand to adjust the smoothing algorithm based on personal experience and preference. The relationship among scan density, spot size, and noise reduction were examined for a planar surface to determine the optimal smoothing window size and number of repetitions for different scan densities. It was discovered that when the scan 
density is larger than the spot size then a small window with few repetitions will minimize the residual error of fitting a plane to measurements of a known planar surface. In fact, for scan density-to-spot size ratios greater than one, a $7 \times 7$ filter window with no more than two repetitions is sufficient. For scan density-to-spot size ratios less than one, the size of the filter window and number of repetitions increases with a decrease in scan density-to-spot size ratio. A future study could examine this effect in detail by more precisely relating beam footprint area to scan density at a variety of ranges.

An issue that has not been well addressed in contemporary research is how to determine the overall quality of a scanning viewpoint. Klein and Sequeira [43] and Klein and Zachmann [104] combined surface orientation, sampling density, and the desired surface resolution into a single quality metric. The approach, however, did not take into account measurement resolution or measurement uncertainty. In this study, resolvability and orientation quality metrics were used to generate a quality-weighted spot size that was then used in an iterative process to automatically find the locally optimal scanner viewpoint. The resolvability quality metric not only quantified whether the beam footprint was small enough to resolve surface features to the desired sampling resolution, but also took into account rotational uncertainty. Experiments with the five test surfaces indicate that the method can be used with a minimally trained operator to find a local optimum in no more than three scanner viewpoint adjustments. This approach used a fast low-density scan to determine the proportion of measurements that would be discarded if the current viewpoint were used to perform high resolution scanning. A model of the scanner viewpoint was then virtually moved until the resolvability and orientation quality metrics were maximized while minimizing the size of the beam footprint. The operator was then instructed how far to move the scanner and by how much to adjust the scanner orientation to reach this locally optimal position. The current and predicted quality weighted spot size were then displayed to give the operator an impression of the degree of improvement that could potentially be realized by moving the scanner viewpoint. 
In this study, planarity, orientation, reflectivity, and resolvability quality metrics were used to automatically divide the total field of view into three regions based on the usefulness of the measurement data that would be obtained from them. Non-return measurements, as well as measurements with unacceptable (0) resolvability and orientation quality were classified as likely to generate measurements that would be discarded during post processing so indicated which regions should be avoided during high-density scanning. Measurements with acceptable (1) planarity quality, no low-outlier quality measurements, and no reflectivity transitions were classified as likely to generate measurements that would be redundant for modeling purposes so indicated which regions should be ignored during high-density scanning. The remaining regions of the total field of view were marked for scanning using a series of small, high-density scan patterns. The regions marked for high-density scanning included the transition between return and non-return measurements where mixed measurements are likely to arise. Experiments using each of the five test surfaces indicate that planar regions are accurately detected and excluded from the region marked for rescanning. Experiment \#5, in particular, demonstrates that surfaces with complex return signal profiles are also detected and included in the region marked for rescanning. Experiment \#2 demonstrates that even shallow range transitions are isolated using the outlier quality metric to identify regions that change significantly after smoothing. Regions with low outlier quality indicate surface regions that could vary significantly from the final model so should be scanned at high resolution to ensure that they are accurately represented.

Once a region of interest has been identified using quality metrics, a sequence of high-density subscans is automatically generated to scan the region. The scan density is automatically set based on the target resolution and/or beam footprint size and includes a margin of error based on the expected rotational uncertainty. Experiments using the five test surfaces indicate that the total scanning time, excluding processing time, is significantly lower than if a single high-density scan were performed of the total field of 
view, or even if the field of view were reduced to encompass the silhouette of the surface being scanned. Moreover, quality maps provide the operator with an indication of not only the total quality of the measurements, but of the reasons why certain measurements may be of lower quality. For example, the orientation quality map warns of high-orientation regions.

The aliasing quality metric is used to determine whether the scan density is sufficient to minimize the chance of aliasing with respect to the target surface sampling resolution. Specifically, if measurements are unacceptably (0) aliased then the subscan density is increased. In this way, the scan density is automatically adapted to the observed intermeasurement surface distance so that the operator does not have to check for aliasing. Moreover, the aliasing quality map reveals problem regions that the operator may wish to rescan or seek the advice of a more experienced operator. Experiments using one of the test surfaces indicate that aliasing can be minimized within a few iterations. Experimental results demonstrate that the total scan time using automatic adaptation to aliasing is still significantly less than scanning the total field of view using a high-density scan.

After a single set of subscans has been performed, the degree of non-coverage of the rescan region is examined and is used to automatically adjust the degree of overlap among subscans. Experiments using the five test surfaces indicate that the total scanning time after a second set of subscans is used to scan the unscanned regions is still less than the total scanning time of a single high-density scan. Experiments using the test surface from Experiment \#3 indicate that a degree of overlap can be obtained within relatively few iterations that eliminates rescan region non-coverage, and that the total scanning time using the new degree of overlap is still less than the total scanning time for a single high-density scan. This result holds even when automatic anti-aliasing and automatic non-coverage correction are combined. 


\subsubsection{Quality-based Merging}

In Chapter 4, a measurement merge method was described in which both the quality of each measurement and their covariance were combined to determine the spatial location and reflectivity of each point in the composite range image. The total quality metric was used to represent the overall quality of each measurement. The $\chi^{2}$ rotational distance between measurement pairs was used to determine whether they should be flagged for merger because they are statistically likely to arise from nearly the same surface region. Flagged measurements were then combined to form a single point in the composite range image. Measurement pairs in which only one measurement had an unacceptable (0) total quality resulted in the unacceptable measurement being dropped. Measurement pairs in which both measurements were of unacceptable quality $(0)$ were combined using the maximum likelihood estimator approach. Measurement pairs in which both were of acceptable $(>0)$ quality were combined using a variation of the maximum likelihood estimator approach in which the spatial and intensity values were weighted toward both the smaller covariance and the higher total quality. Where measurement covariance was approximately equal, the spatial and intensity values would be most similar to the measurement of higher total quality. Measurements of significantly lower covariance are the result of previous merges so spatial and intensity values are more similar to the previously merged value unless the new measurement is of significantly higher quality.

The quality-weighted merge procedure was designed to handle the situation of a non-return subscan measurement being rotationally close to a return anchor scan measurement. In this case, the anchor scan measurement is a result of a long integration region so the rotational position of the measurement may not correspond to a real surface. Subscan measurements, arising from a much shorter integration region, are more accurate measurements of the presence or absence of a reflective surface. As a result, it is possible to have a non-return subscan measurement from a region in the total field of view that 
rotationally corresponds to a return anchor scan measurement. The merge procedure automatically discards any anchor scan measurement that is rotationally close to a nonreturn subscan measurement. This reduces the likelihood of false edges being included in the composite range image. Many of the quality metrics developed or improved in this study for raster scans were adapted for non-grid data sets such as arise from merging multiple scans. Measurements that share a Delaunay edge with a measurement were defined as its neighbours for purposes of generating quality metrics for points in the composite range image. Reflectivity, orientation, and aliasing metrics were defined for composite range image points. As well, a repeatability quality metric was developed in this study to represent the quality of points in a composite range image based on the consistency of range measurement values.

\section{$5.2 \quad$ Future Work}

The current study was a proof-of-concept for using adaptive scans and quality maps for improving model fidelity while minimizing the time spent acquiring data. Many issues have not been addressed in this study such as motion distortion, view planning, and how best to merge data from other scanner viewpoints. The latter problem can be further divided into problems of representing measurement quality using merged data and minimizing registration error.

\subsubsection{Motion Distortion}

The system envisioned within the context of this research consists of a laser range scanner that is placed in a stable location, such as on the ground or on a platform, prior to scanning a surface. This should avoid motion distortion caused by hand shaking that is an issue in hand-held laser range scanners [152]. Contemporary hand-held systems must 
resort to registration techniques such as ICP to compensate for motion distortion [5] [152]-[154]; however, techniques like ICP depend on identifying correspondences among scans [5] [152] [153] [155] [156] so typically require significant measurement redundancy. This redundancy increases the time required to scan a surface.

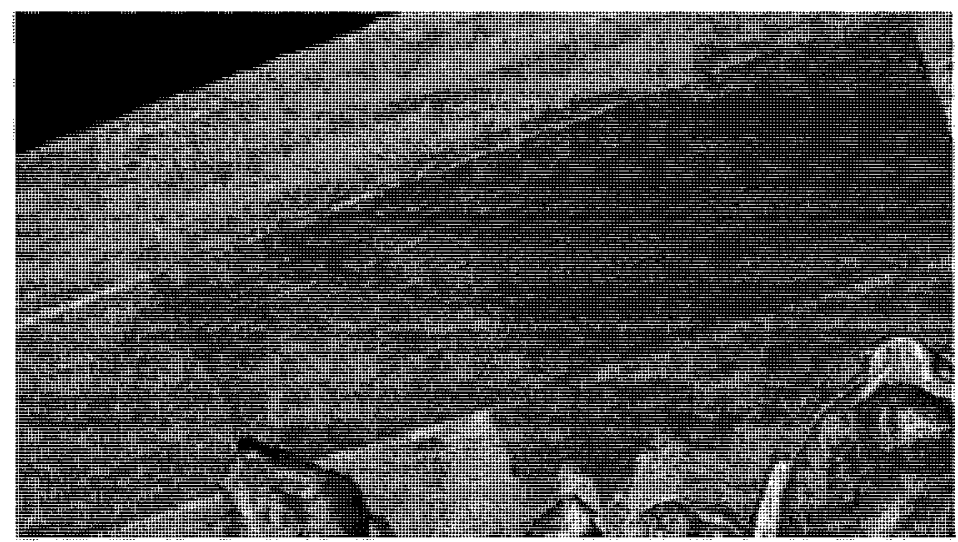

Figure 5.1: Motion distortion can result in wave patterns such as those shown in this range image (Reproduced from Figure 5 of [41]).

Blais [5] [153] suggested that even relatively stable platforms can become corrupted with low-frequency noise of between 10 and $100 \mathrm{~Hz}$ [153] during a single scan. Techniques are required to compensate for low-frequency motion distortion, either using detection and feedback or introducing an additional uncertainty term, such as an aperiodic measurement confirmation (AMC). An AMC technique could involve randomly resampling the surface to determine whether measurement pair variance was greater than could be accounted for by sensor variance alone. Another approach is to select a landmark that is aperiodically resampled throughout the scanning process to detect low-frequency changes in measurements at levels greater than the level of sensor noise. A similar technique was employed by Blais to track a surface [5] [153]; however their technique involved tracking the entire surface rather than one or more static features. 


\subsubsection{Merged Measurement Map}

It was assumed in this study that no previous measurements had been obtained from other viewpoints. This meant that all measurements could be assumed to have been obtained from the current scanner viewpoint so were spatially limited by the scanner resolution; that is, their inter-measurement distance would be limited by the resolution of the scanning system. If multiple viewpoints were included then the inter-measurement distance would be smaller for viewpoints in which the beam waist was closer to the surface. Moreover, registration of scans from multiple viewpoints introduces additional measurement error that must be included in the covariance matrix [157] [158]. These differences in measurement resolution and registration errors would require the development of additional quality metrics. Future work should examine techniques for including measurements from other viewpoints when constructing quality maps for the current viewpoint, and should examine how to represent the global, rather than within-viewpoint, quality of measurements.

\subsubsection{Registration Error}

Combining laser range scanner measurements from multiple viewpoints into a single virtual model involves matching, registration and integration of these measurements [159][162]. A typical approach is to use control points which must correspond among range images [160] [161] [163] [164], and may even have been independently surveyed so that their spatial location is known to a high degree of accuracy [23] [165] [166]. If the position and orientation of each viewpoint could be known within a global frame of reference to a high degree of accuracy then the registration error would be minimized [167]. One way to accurately determine the current orientation of the scanner is to use landmarks as a reference [168]. Assuming the scene is static and the scanner is not moving, then triplets of landmarks [168] can be used between viewpoints to determine the scanner's 
relative position and orientation. This technique is often used by robots to determine their location in an environment [169], and more recently for simultaneous localization and mapping (SLAM) [170] [171].

Often researchers use landmarks, either natural [24] [158] [172] or artificial [23] [162] [173], to improve range image registration. Methods exist for automatically identifying [168] [174] and evaluating [168] [169] landmarks; however, a human operator can evaluate the results of automatic landmark selection and possibly suggest alternative landmarks based on where they plan to start scanning next. If a requirement for any new viewpoint is that at least three landmarks must be visible from that viewpoint, then the choice of new viewpoints is limited. Similarly, if an operator can eliminate viewpoints that are not feasible then the choice of landmarks is limited to those visible from feasible locations. A result of this interactive relationship should be to improve both the quality of landmarks selected and to reduce the registration error. Future research would explore using an interactive approach to landmark selection and identification, and would determine whether this approach could reduce registration error.

\subsubsection{Edge Distortion}

Non-return measurements are generally ignored in contemporary research; however, they indicate locations in the total field of view in which no surface information can be obtained. As indicated in Section 2.4, many factors can affect the strength of the return signal so its absence does not necessarily indicate the absence of a surface, merely the absence of an indication that the surface exists. In this study, the absence of a return signal is taken as an indication of a region that should be examined in greater detail if even one of its neighbours generates a return signal. Moreover, the rotational position of the non-return measurement provides a boundary to the region that should be examined in detail. 
Surface discontinuities present a special problem for laser range scanners because they can result in a laser signal being split between two surfaces [2] [3] [51] [71] [76] [77] [86] [159] [175]. Witzgall [159] examined the effect of edges on the construction of accurate Delaunay mesh surfaces but focused on post-processing of laser range scanner data. Tuley [86] focused on identifying and filtering out mixed pixels that result from edges. Future research can address how to automatically perform super-resolution scanning of mixed-measurement regions, implementing some of the approaches currently being examined for obtaining more accurate transition region information. For example, ultrahigh density scanning could be combined with a model of the return signal profile to correct for signal profile truncation, which can then be used to correct for range errors.

\subsubsection{View Planning}

View planning is an active area of research [1]-[4] [9] [42] [43] [54] [108] [110] [112] [176][192], but few have used quality metrics to assist in the planning process. Sequeira et al. used quality metrics for next-best-view planning, while Callieri et al. used a multistage approach, first developed by Scott et al. [42] [54] [110] [177]-[181] for small-volume scanning, in which an initial low-density scan is followed by a series of high-density targeted scans. Both Callieri et al. and Scott et al. used a form of quality analysis referred to as a measurability matrix in which future views were selected based on the likelihood of acquiring sufficient useful information from a particular viewpoint.

One approach to quality-based view planning is to divide the surface model into two regions: the region in which the orientation and resolvability quality are currently acceptable, and the remainder of the surface model. The former region is referred to as the Completed region and the latter is referred to as the Rescan region. A series of viewpoints would then be sought that maximize the orientation and resolvability quality of the Rescan region. After each optimal viewpoint is selected, the regions of the model 
likely to generate acceptable-quality measurements are classified as Targetted so that the next viewpoint can be obtained that maximizes the orientation and resolvability quality of the portion of the Rescan region not already marked as Targetted. Operator feedback can be used to eliminate viewpoints that it are not feasible to attain, resulting in a reanalysis of the Rescan region. Reanalysis of the Rescan region would also take place after each composite range image is merged into the model. This approach is best suited to medium- and large-volume scanning of environments rather than single objects because it requires a minimum of scanner repositionings. The problem with this approach is that the number of data points in the model quickly becomes very large so data reduction and model rescaling techniques would be required. This approach is also best suited to operator-assisted scanning because the operator is able to quickly see the completed model emerge from the scanning process.

An alternative approach is one advocated by Scott et al. [42] [54] [110] [177]-[181] in which a series of low-density scans are performed of the entire environment to determine the measurability of all surfaces to be included in the model. The environment is then rescanned using high-density scan patterns, following the sequence of viewpoints obtained from an analysis of the measurability of all surfaces. Surface measurability can be augmented with the quality metrics presented in this thesis, and the process of determining subscans would be replaced with a combination of view planning and subscan generation. The quality-weighted spot size approach would still be employed to seek locally optimal viewpoints, but the optimal anchor scans would then be used to ensure complete surface coverage in which all low-density scans exhibit locally-optimal orientation and resolvability quality. This approach is best suited to small- and mediumvolume scanning of single objects or object clusters because high-density scanning would be based on a complete evaluation of the surface or surfaces being scanned. The problem with this approach is that the number of scanner repositionings can be very large if the 
surface area being scanned is large or the surface is complex, but this is not a serious issue if the scanning system is automated rather than operator-guided. 


\section{References}

[1] V. Sequeira, J. G. Goncalves, and M. I. Ribeiro, "3D Scene modelling from multiple range views," in Proceedings of the SPIE: Videometrics IV, S. F. El-Hakim, Ed., vol. 2598, Philadelphia, Pennsylvania, USA, 25-26 October 1995, pp. 114-127.

[2] V. Sequeira, K. Ng, E. Wolfart, J. G. Goncalves, and D. Hogg, "Automated 3D reconstruction of interiors with multiple scan-views," in Proceedings of SPIE, vol. 3641, 1998, pp. 106-117.

[3] V. Sequeira, K. Ng, E. Wolfart, J. G. M. Goncalves, and D. Hogg, "Automated reconstruction of 3D models from real environments," ISPRS Journal of Photogrammetry and Remote Sensing, vol. 54, no. 1, pp. 1-22, February 1999.

[4] V. Sequeira and J. Goncalves, "3D reality modelling: photo-realistic 3D models of real world scenes," in Proceedings of the First International Symposium on $3 D$ Data Processing Visualization and Transmission, 19-21 June 2002, pp. 776-783.

[5] F. Blais, M. Picard, and G. Godin, "Recursive Model Optimization Using ICP and Free Moving 3D Data Acquisition," in Proceedings of the 4th International Conference on 3-D Digital Imaging and Modeling, Banff, AL, Canada, 6-10 October 2003. 
[6] G. Godin, J.-A. Beraldin, J. Taylor, L. Cournoyer, M. Rioux, S. El-Hakim, R. Baribeau, F. Blais, P. Boulanger, J. Domey, and M. Picard, "Active optical 3D imaging for heritage applications," IEEE Computer Graphics and Applications, vol. 22, no. 5, pp. 24-35, September/October 2002.

[7] P. K. Allen, A. Troccoli, B. Smith, S. Murray, I. Stamos, and M. Leordeanu, "New Methods for Digital Modeling of Historic Sites," IEEE Computer Graphics and Applications, vol. 23, no. 6, pp. 32-41, November/December 2003.

[8] P. K. Allen, I. Stamos, A. Troccoli, B. Smith, M. Leordeanu, and Y. C. Hsu, "3D Modeling of Historic Sites using Range and Image Data," in Proceedings of International Conference on Robotics and Animation. Taipei, Taiwan: IEEE, 1119 September 2003, pp. 145-150.

[9] H. Surmann, A. Nuchter, and J. Hertzberg, "An autonomous mobile robot with a 3D laser range finder for 3D exploration and digitalization of indoor environments," Robotics and Autonomous Systems, vol. 45, no. 3-4, pp. 181-198, December 2003.

[10] I. Kweon and T. Kanade, "High-resolution terrain map from multiple sensor data," IEEE Transactions on Pattern Analysis and Machine Intelligence, vol. 14, no. 2, pp. 278-292, February 1992.

[11] F. Blais, J.-A. Beraldin, S. El-Hakim, and L. Cournoyer, "Real-Time Geometrical Tracking and Pose Estimation Using Laser Triangulation and Photogrammetry," in Proceedings of the Third International Conference on 3D Digital Imaging and Modeling, Québec City, QC, Canada, May 28 - June 12001.

[12] P. Payeur, D. Laurendeau, and C. Gosselin, "Range data merging for probabilistic octree modeling of 3D workspaces," in Proceedings of the IEEE International Conference on Robotics and Automation, vol. 4, 1998, pp. 3071-3078. 
[13] P. Payeur, "3D modeling from range images with integrated registration," in Proceedings of the IEEE International Workshop on Haptic Virtual Environments and Their Applications, 17-18 November 2002, pp. 79-84.

[14] — - "Dealing with uncertain measurements in virtual representations for robot guidance," in Proceedings of the IEEE International Symposium on Virtual and Intelligent Measurement Systems, 2002, pp. 56-61.

[15] J.-A. Beraldin, F. Blais, M. Rioux, L. Cournoyer, D. Laurin, and S. MacLean, "Eye-safe digital 3-D sensing for space applications," Optical Engineering, vol. 39, no. 1 , pp. 196-211, January 2000.

[16] S. El-Hakim, P. Boulanger, F. Blais, J.-A. Beraldin, and G. Roth, "A Mobile System for Indoors 3-D Mapping and Positioning," in Proceedings of Optical 3D Measurement Techniques IV, Zurich, Switzerland, September 1997, pp. 275-282.

[17] I. Heinz, F. Hartl, and C. Frohlich, "Semi-automatic 3D CAD model generation of as-built conditions of real environments using a visual laser radar," in Proceedings of the IEEE International Workshop on Robot and Human Interactive Communication, Bordeaux, Paris, France, 18-21 September 2001, pp. 400-406.

[18] R. Roncella, G. Forlani, and F. Remondino, "Photogrammetry for geological applications: automatic retrieval of discontinuity orientation in rock slopes," in Proceedings of the SPIE: Videometrics VIII, J.-A. Beraldin, S. F. El-Hakim, A. Gruen, and J. Walton, Eds., vol. 5665, San Jose, California, USA, 18-20 January 2005, pp. 17-27.

[19] A. Deslauriers, I. Showalter, A. Montpool, R. Taylor, and I. Cristie, "Shuttle TPS Inspection using Triangulation Scanning Technology," in Proceedings of the SPIE: 
Airborne Sensors II, P. T. Jr. and B. Holz, Eds., vol. 5798, Orlando, Florida, USA, 28-29 March 2005, pp. 26-33.

[20] M. Mettenleiter, F. Härtl, C. Fröhlich, and D. Langer, "Imaging Laser Radar for 3-D Modelling of Real World Environments," in Proceedings of the International Conference on OPTO / IRS2 / MTT, 9-11 May 2000.

[21] R. Singh, D. Chapman, and K. Atkinson, "CCD Based active triangulation for rock surface measurements," in Proceedings of the SPIE: Videometrics IV, S. F. El-Hakim, Ed., vol. 2598, Philadelphia, Pennsylvania, USA, 25-26 October 1995, pp. 233-244.

[22] S. Slob, H. Hack, and A. Turner, "An approach to automate discontinuity measurements of rock faces using laser scanning techniques," in Proceedings of the ISRM International Symposium on Rock Engineering for Mountainous Regions, C. D. da Gama and L. R. e Sousa, Eds. Sociedade Portuguesa de Geotecnia, 25-28 November 2002, pp. 87-94.

[23] Q. Feng, "Novel methods for 3-D semi-automatic mapping of fracture geometry at exposed rock faces," PhD Thesis, Royal Institute of Technology, Stockholm, Sweden, May 2001.

[24] C. Chao and I. Chao, "Semi-Automatic Range to Range Registration: A FeatureBased Method," in Proceedings of the Fifth International Conference on 3-D Digital Imaging and Modeling, 13-16 June 2005, pp. 254-261.

[25] I. Abuhadrous, F. Nashashibi, C. Laurgeau, and F. Goulette, "Onboard real-time system for 3D urban environment reconstruction," in Proceedings of the IEEE Intelligent Vehicles Symposium, 9-11 June 2003, pp. 479-483. 
[26] G. J. Iddan and D. G. Yahav, "3D Imaging in the studio," in Proceedings of the SPIE: Three-Dimensional Image Capture and Applications IV, B. D. Corner, J. H. Nurre, and R. P. Pargas, Eds., vol. 4298, April 2001, pp. 48-55.

[27] L. Nyland, D. McAllister, V. Popescu, C. McCue, A. Lastra, P. Rademacher, M. Oliveira, G. Bishop, G. Meenakshisundaram, M. Cutts, and H. Fuchs, "The impact of dense range data on computer graphics," in Proceedings of the IEEE Workshop on Multi-View Modeling and Analysis of Visual Scenes, Fort Collins, CO, USA, 26 June 1999, pp. 3-10.

[28] S. F. El-Hakim, P. Boulanger, F. Blais, and J.-A. Beraldin, "A system for indoor 3-D mapping and virtual environments," in Proceedings of the SPIE: Videometrics $V$, vol. 3174 , July 1997 , p. 2135.

[29] S. F. El-Hakim, C. Brenner, and G. Roth, "A multi-sensor approach to creating accurate virtual environments," ISPRS Journal of Photogrammetry and Remote Sensing, vol. 53, no. 6, pp. 379-391, December 1998.

[30] J.-A. Beraldin, F. Blais, L. Cournoyer, M. Rioux, F. Bernier, and N. Harrison, "Portable digital 3-D imaging system for remote sites," in Proceedings of the IEEE International Symposium on Circuits and Systems, vol. 5, 31 May-3 June 1998, pp. $488-493$.

[31] J.-A. Beraldin, M. Picard, S. El-Hakim, G. Godin, V. Valzano, A. Bandiera, and D. Latouche, "Virtualizing a Byzantine Crypt by Combining High-resolution Textures with Laser Scanner 3D Data," in Proceedings of Virtual Systems and Multimedia, Gyeongju, Korea, September 25-27 2002, pp. 3-14.

[32] J.-A. Beraldin, M. Picard, S. El-Hakim, G. Godin, E. Paquet, S. Peters, M. Rioux, V. Valzano, and A. Bandiera, "Virtual heritage: the cases of the byzantine crypt of 
Santa Cristina and temple C of Selinunte," in Proceedings of Virtual Systems and Multimedia, Japan, 17-19 November 2004.

[33] J.-A. Beraldin, M. Picard, S. El-Hakim, G. Godin, L. Borgeat, F. Blais, E. Paquet, M. Rioux, V. Valzano, and A. Bandiera, "Virtual Reconstruction of Heritage Sites: Opportunities and Challenges Created by 3D Technologies," in Proceedings of the International Workshop on Recording, Modeling and Visualization of Cultural Heritage, Ascona, Switzerland, 22-27 May 2005.

[34] J.-A. Beraldin, M. Picard, S. El-Hakim, G. Godin, V. Valzano, and A. Bandiera, "Combining 3D technologies for cultural heritage interpretation and entertainment," in Proceedings of the SPIE: Videometrics VIII, J.-A. Beraldin, S. F. ElHakim, A. Gruen, and J. Walton, Eds., vol. 5665, San Jose, California, USA, 18-20 January 2005 , pp. 108-118.

[35] G. Guidi, F. B., M. De Simone, A. Cioci, A. Spinetti, L. Carosso, L. Micoli, M. Russo, and T. Grasso, "Virtualizing ancient Rome: 3D acquisition and modeling of a large plaster-of-Paris model of imperial Rome," in Proceedings of the of the SPIE - Videometrics VIII, vol. 5665, San Jose, USA, 18-20 January 2005, pp. 119 133.

[36] F. Dekeyser, F. Gaspard, L. de Luca, M. Florenzano, X. Chen, and P. Leray, "Cultural Heritage Recording with Laser Scanning, Computer Vision and Exploitation of Architectural Rules," in The International Archives of the Photogrammetry, Remote Sensing and Spatial Information Sciences, vol. XXXIV, no. 5/W12. Corfu, Greece: ISPRS, 2-6 September 2002.

[37] S. El-Hakim, J.-A. Beraldin, M. Picard, and A. Vettore, "Effective 3D Modeling of Heritage Sites," in Proceedings of the 4th International Conference of $3 D$ Imaging and Modeling, Banff, AL, Canada, 6-10 October 2003, pp. 302-309. 
[38] M. Levoy, K. Pulli, B. Curless, S. Rusinkiewicz, D. Koller, L. Pereira, M. Ginzton, S. Anderson, J. Davis, J. Ginsberg, J. Shade, and D. Fulk, "The Digital Michelangelo Project: 3D Scanning of Large Statues," in Proceedings of SIGGRAPH, 2000, pp. 131-144.

[39] J. Taylor and J.-A. Beraldin, "Heritage Recording Applications of High Resolution 3D Imaging," in Proceedings of the Electronic Imaging and the Visual Arts, Florence, Italy, March 26-30 2001.

[40] C. Teutsch, T. Isenberg, E. Trostmann, M. Weber, D. Berndt, and T. Strothotte, "Evaluation and Correction of Laser-Scanner Point Clouds," in Proceedings of the SPIE: Videometrics VIII, J.-A. Beraldin, S. F. El-Hakim, A. Gruen, and J. Walton, Eds., vol. 5665, San Jose, California, USA, 18-20 January 2005, pp. 172-183.

[41] J.-A. Beraldin, "Integration of Laser Scanning and Close-Range Photogrammetry - The Last Decade and Beyond," in Proceedings of the XXth International Society for Photogrammetry and Remote Sensing (ISPRS) Congress. Commission VII, Istanbul, Turkey, 12-23 July 2004, pp. 972-983.

[42] W. R. Scott, G. Roth, and J.-F. Rivest, "View planning for automated threedimensional object reconstruction and inspection," ACM Computing Surveys, vol. 35, no. 1, pp. 64-96, March 2003.

[43] K. Klein and V. Sequeira, "The view-cube: an efficient method of view planning for 3D modelling from range data," in Proceedings of the Fifth IEEE Workshop on Applications of Computer Vision, Palm Springs, CA, USA, 4-6 December 2000, pp. 186-191. 
[44] F. Chen, G. M. Brown, and M. Song, "Overview of three-dimensional shape measurement using optical methods," Optical Engineering, vol. 39, no. 1, pp. 10-22, January 2000.

[45] G. Cheok, W. Stone, and A. Lytle, "Standards Requirements for LADARs?" in Proceedings of SPIE: Aerosense, 2005.

[46] H. Zhang, O. Hall-Holt, and A. Kaufman, "Range image registration via probability field," in Proceedings of Computer Graphics International, 2004, pp. 546-552.

[47] F. Blais, J. A. Beraldin, and S. F. El-Hakim, "Range Error Analysis of an Integrated Time-of-flight, Triangulation, and Photogrammetry 3D Laser Scanning System," in Proceedings of the SPIE: AeroSense, G. W. Kamerman, U. N. Singh, C. Werner, and V. V. Molebny, Eds., vol. 4035, no. 1, Orlando, FL., September 2000, pp. 236-247.

[48] J. G. Walker, "Optical imaging with resolution exceeding the Rayleigh criterion," Optica Acta, vol. 30, no. 9, pp. 1197-1202, 1983.

[49] D. D. Lichti and S. Jamtsho, "Angular resolution of terrestrial laser scanners," The Photogrammetric Record, vol. 21, no. 114, pp. 141-160, June 2006.

[50] F. Blais and J.-A. Beraldin, "Recent developments in 3D multi-modal laser imaging applied to cultural heritage," Machine Vision and Applications, vol. 17, no. 6, pp. 395-409, 2006.

[51] M. Hebert and E. Krotkov, "3-D measurements from imaging laser radars: how good are they?" in Proceedings of the IEEE/RSJ International Workshop on Intelligent Robots and Systems, vol. 1, 3-5 November 1991, pp. 359-364.

[52] F. Blais, J. Taylor, L. Cournoyer, M. Picard, L. Borgeat, L. Dicaire, M. Rioux, J.-A. Beraldin, G. Godin, C. Lahanier, and G. Aitken, "High resolution imaging at 50 
$\mu \mathrm{m}$ using a portable XYZ-RGB color laser scanner," in International Workshop on Recording, Modeling and Visualization of Cultural Heritage, Centro Stefano Franscini, Monte Verita. Ascona, Switzerland, 22-27 May 2005.

[53] B. Rohani and H.-J. Zepernick, "Application of a perceptual speech quality metric for link adaptation in wireless systems," in Proceedings of the 1st International Symposium on Wireless Communication Systems, 20-22 September 2004, pp. 260264.

[54] W. Scott, G. Roth, and J. Rivest, "Performance-Oriented View Planning for Model Acquisition," in Proceedings of the The International Symposium on Robotics, Montréal, QC, Canada, May 2000, pp. 212-219.

[55] J. S. Ryan and A. I. Carswell, "Laser beam broadening and depolarization in dense fogs," Journal of the Optical Society of America, vol. 68, no. 7, pp. 900-908, July 1978.

[56] J.-A. Beraldin, S. El-Hakim, and L. Cournoyer, "Practical range camera calibration," in Proceedings of the SPIE: Videometrics II, vol. 2067, Boston, Massachusetts, 7-10 September 1993, pp. 21-31.

[57] M. Adams, "Lidar design, use, and calibration concepts for correct environmental detection," IEEE Transactions on Robotics and Automation, vol. 16, no. 6, pp. 753-761, December 2000.

[58] D. Green and F. Blais, "A Multiple DSP-based 3D Laser Range Sensor and its Application to Real-time Motion Detection," National Research Council of Canada, Tech. Rep. NRC/ERB-1095, May 2002.

[59] D. MacKinnon, F. Blais, and V. Aitken, "Object Location using Edge-Bounded Planar Surfaces from Sparse Range Data," in Proceedings of the Canadian Con- 
ference on Electrical and Computer Engineering, vol. 1, 4-7 May 2003, pp. 403408.

[60] M. Longbin, S. Ziaoquan, Z. Yiyu, and S. Z. Chang, "Unbiased Converted Measurements for Tracking," IEEE Transactions on Aerospace and Electronic Systems, vol. 34, no. 3, pp. 1023-1027, July 1998.

[61] P. Suchomski, "Explicit Expressions for Debiased Statistics of 3D Converted Measurements," IEEE Transactions on Aerospace and Electronic Systems, vol. 35, no. 1, pp. 368-370, January 1999.

[62] J.-A. Beraldin, C. Latouche, S. El-Hakim, and A. Filiatrault, "Applications of Photogrammetric and Computer Vision Techniques in Shake Table Testing," in Proceedings of the 13th World Conference on Earthquake Engineering, Vancouver, BC, Canada, 1-6 August 2004.

[63] F. Blais, "Review of 20 Years of Range Sensor Development," Journal of Electronic Imaging, vol. 13, no. 1, pp. 231-243, January 2004.

[64] M. Adams, "Coaxial range measurement - current trends for mobile robotic applications," IEEE Sensors Journal, vol. 2, no. 1, pp. 2-13, February 2002.

[65] M.-C. Amann, T. Bosch, M. Lescure, R. Myllyla, and M. Rioux, "Laser ranging: a critical review of usual techniques for distance measurement," Optical Engineering, vol. 40, no. 1, pp. 10-19, January 2001.

[66] E. Garcia and H. Lamela, "Low-cost three-dimensional vision system based on a low-power semiconductor laser rangefinder and a single scanning mirror," Optical Engineering, vol. 40, no. 1, pp. 61-66, January 2001.

[67] B. L. Curless, "New methods for surface reconstruction from range images," $\mathrm{PhD}$ Thesis, Stanford University, 1997. 
[68] R. Baribeau and M. Rioux, "Influence of speckle on laser range finders," Applied Optics, vol. 30, no. 20, pp. 2873-2978, July 1991.

[69] J. W. Goodman, "Some fundamental properties of speckle," Journal of the Optical Society of America, vol. 66, no. 11, pp. 1145-1150, November 1976.

[70] R. Baribeau, M. Rioux, and G. Godin, "Color reflectance modeling using a polychromatic laser range sensor," IEEE Transactions on Pattern Analysis and Machine Intelligence, vol. 14, no. 2, pp. 263-269, February 1991.

[71] J. A. Hancock, "Laser Intensity-Based Obstacle Detection and Tracking," PhD Dissertation, The Robotics Institute, Carnegie Mellon University, January 1999.

[72] F. Prieto, P. Boulanger, R. Lepage, and T. Redarce, "Automated inspection system using range data," in Proceedings of the IEEE International Conference on Robotics and Automation, vol. 3, Washington, DC, USA, 11-15 May 2002, pp. 2557-2562.

[73] J. Lang and D. Pai, "Bayesian estimation of distance and surface normal with a time-of-flight laser rangefinder," in Proceedings of the Second International Conference on 3-D Digital Imaging and Modeling, Ottawa, ON, CAN, 4-8 October 1999, pp. 109-117.

[74] M. Soucy and D. Laurendeau, "A general surface approach to the integration of a set of range views," IEEE Transactions on Pattern Analysis and Machine Intelligence, vol. 17, no. 4, pp. 344-358, April 1995.

[75] A. Johnson, R. Hoffman, J. Osborn, and M. Hebert, "A system for semi-automatic modeling of complex environments," in Proceedings of the International Conference on Recent Advances in 3-D Digital Imaging and Modeling, Ottawa, ON, Canada, 12-15 May 1997, pp. 213-220. 
[76] J. Hancock, M. Hebert, and C. Thorpe, "Laser intensity-based obstacle detection," in Proceedings of the IEEE/RSJ International Conference on Intelligent Robots and Systems, vol. 3, Victoria, BC, Canada, 13-17 October 1998, pp. 1541-1546.

[77] J. Hancock, D. Langer, M. Hebert, R. Sullivan, D. Ingimarson, E. Hoffman, M. Mettenleiter, and C. Froehlich, "Active laser radar for high-performance measurements," in Proceedings of the IEEE International Conference on Robotics and Automation, vol. 2, Leuven, Belgium, 16-20 May 1998, pp. 1465-1470.

[78] J.-A. Beraldin, F. Blais, M. Rioux, J. Domey, L. Gonzo, F. D. Nisi, F. Comper, D. Stoppa, M. Gottardi, and A. Simoni, "Optimized Position Sensors for FlyingSpot Active Triangulation Systems," in Proceedings of the Fourth International Conference on 3-D Digital Imaging and Modeling, Banff, AL, Canada, 6-10 October 2003, pp. 29-36.

[79] G. Godin, J.-A. Beraldin, M. Rioux, M. Levoy, and L. Cournoyer, "An Assessment of Laser Range Measurement of Marble Surfaces," in Proceedings of the 5th Conference on Optical 3-D Measurement Techniques, Vienna, Austria, 1-4 October 2001, pp. 49-56.

[80] S. El-Hakim and J.-A. Beraldin, "Configuration design for sensor integration," in Proceedings of the SPIE: Videometrics IV, vol. 2598, Philadelphia, Pennsylvania, 22-26 October 1995, pp. 274-285.

[81] F. DeNisi, F. Comper, L. Gonzo, M. Gottardi, D. Stoppa, A. Simoni, and J.-A. Beraldin, "A CMOS Sensor Optimized for Laser Spot-Position Detection," IEEE Sensors Journal, vol. 5, no. 6, pp. 1296-1304, December 2005. 
[82] M. Rutishauser, M. Stricker, and M. Trobina, "Merging range images of arbitrarily shaped objects," in Proceedings of the IEEE Computer Society Conference on Computer Vision and Pattern Recognition, 21-23 June 1994, pp. 573-580.

[83] Z. Zhang and O. Faugeras, "A 3D world model builder with a mobile robot," International Journal of Robotics Research, vol. 11, no. 4, pp. 269-285, August 1992.

[84] D. Carmer and L. Peterson, "Laser radar in robotics," Proceedings of the IEEE, vol. 84, no. 2, pp. 299-320, February 1996.

[85] S. El-Hakim and J.-A. Beraldin, "On the Integration of Range and Intensity Data to Improve Vision-based Threee-Dimensional Measurements," in Proceedings of the SPIE: Videometrics III, S. F. El-Hakim, Ed., vol. 2350, Boston, Massachusetts, USA, 2-4 November 1994, pp. 306-321.

[86] J. Tuley, N. Vandapel, and M. Hebert, "Analysis and Removal of artifacts in 3-D LADAR Data," in Proceedings of the IEEE International Conference on Robotics and Automation, January 2005, pp. 2203-2210.

[87] G. Godin, M. Rioux, and R. Baribeau, "Three-dimensional registration using range and intensity information," in Proceedings of the SPIE: Videometrics III, S. F. El-Hakim, Ed., vol. 2350, Boston, Massachusetts, USA, 2-4 November 1994, pp. $279-290$.

[88] M. Fiocco, G. Boström, J. Gonçalves, and V. Sequeira, "Multisensor fusion for Volumetric Reconstruction of Large Outdoor Areas," in Proceedings of the Fifth International Conference on 3-D Digital Imaging and Modeling, 2005, pp. 47-54.

[89] D. Williams, Optical Methods in Engineering Metrology, 1st ed. Chapman \& Hall, 1993, pp. 11-16. 
[90] B. Chu, Laser Light Scattering Basic Principles and Practice, 2nd ed. Academic Press, Inc., 1991, pp. 156-160.

[91] G. Jacobs, "Understanding Spot Size for Laser Scanning," Professional Surveyor Magazine, October 2006.

[92] E. Argyle, "Techniques for Edge Detection," Proceedings of the IEEE, vol. 59, no. 2, pp. 285-287, February 1971.

[93] L. Davis, "A Survey of Edge Detection Techniques," Computer Graphics Image Processing, vol. 4, no. 3, pp. 248-270, September 1975.

[94] T. Peli and D. Malah, "A Study of Edge Detection Algorithms," Computer Graphics Image Processing, vol. 20, pp. 1-21, 1982.

[95] D. Ziou and S. Tabbone, "Edge Detection Techniques - An Overview," International Journal of Pattern Recognition and Image Analysis, vol. 8, pp. 537-559, 1998.

[96] H. Trichili, M.-S. Bouhlel, N. Derbel, and L. Kamoun, "A survey and evaluation of edge detection operators application to medical images," in Proceedings of the IEEE International Conference on Systems, Man and Cybernetics, vol. 4, 6-9 October 2002.

[97] Z. Xiao, M. Yu, C. Guo, and H. Tang, "Analysis and comparison on image feature detectors," in Proceedings of the 3rd International Symposium on Electromagnetic Compatibility, 21-24 May 2002, pp. 651-656.

[98] M. Basu, "Gaussian-based edge-detection methods-a survey," IEEE Transactions on Systems, Man and Cybernetics, Part C, vol. 32, no. 3, pp. 252-260, August 2002. 
[99] Abdul J. Jerri, "The Shannon Sampling Theorem-Its Various Extensions and Applications: A Tutorial Review," Proceedings of the IEEE, vol. 65, no. 11, pp. 1565-1596, November 1977.

[100] C.-H. Lee, "Image surface approximation with irregular samples," IEEE Transactions on Pattern Analysis and Machine Intelligence, vol. 11, no. 2, pp. 206-212, February 1989.

[101] A. V. Oppenheim and R. W. Schafer, Discrete Signal Processing, 2nd ed., ser. Prentice-Hall Signal Processing. Prentice-Hall, Inc., 1999, ch. 4, pp. 142-147.

[102] G. Guidi, B. Frischer, M. Russo, A. Spinetti, L. Carosso, and L. L. Micoli, "Threedimensional acquisition of large and detailed cultural heritage objects," Machine Vision and Applications, 2006.

[103] R. D. Fiete and T. A. Tantalo, "Image quality of increased along-scan sampling for remote sensing systems," Optical Engineering, vol. 38, no. 5, pp. 815-820, May 1999.

[104] J. Klein and G. Zachmann, "Proximity Graphs for Defining Surfaces over Point Clouds," in Proceedings of the Eurographics Symposium on Point-Based Graphics, M. Alexa and S. Rusinkiewicz, Eds., 2004.

[105] A. J. den Dekker and A. van den Bos, "Resolution: a survey," Journal of the Optical Society of America A, vol. 14, no. 3, pp. 547-557, March 1997.

[106] M. Soucy, A. Croteau, and D. Laurendeau, "A multi-resolution surface model for compact representation of range images," in Proceedings of the IEEE International Conference on Robotics and Automation, vol. 2, Nice, France, 12-14 May 1992, pp. $1701-1706$. 
[107] G. Turk and M. Levoy, "Zippered Polygon Meshes from Range Images," in SIGGraph-94, 1994, pp. 311-318.

[108] N. A. Massios and R. B. Fisher, "A Best Next View Selection Algorithm incorporating a Quality Criterion," in 9th British Machine Vision Conference, Southampton, England, 1998, pp. 780-789.

[109] H. Hoppe, T. DeRose, T. Duchamp, J. McDonald, and W. Stuetzle, "Surface Reconstruction from Unorganized Points," in Proceedings of SIGRAPH, vol. 26, July 1992, pp. 71-78.

[110] W. Scott, G. Roth, and J.-F. Rivest, "View Planning for Multi-Stage Object Reconstruction," in Proceedings of Vision Interface, Ottawa, ON, Canada, June 2001, pp. 64-71.

[111] A. Johnson and S. B. Kang, "Registration and integration of textured 3-D data," in Proceedings of the International Conference on Recent Advances in 3-D Digital Imaging and Modeling, 1997, pp. 234-241.

[112] M. Callieri, A. Fasano, G. Impoco, P. Cignoni, R. Scopigno, G. Parrini, and G. Biagini, "RoboScan: an automatic system for accurate and unattended 3D scanning," in Proceedings of the 2nd International Symposium on 3D Data Processing, Visualization and Transmission, 6-9 September 2004, pp. 805-812.

[113] F. Blais, J. Beraldin, and S. El-Hakim, "Real-time 3D Pose Estimation Using Photogrammetry and Laser Based Geometrical Target Tracking for Autonomous Operation in Space," in Proceedings of the 5th Conference on Optical 3-D Measurement Techniques, Vienna, Austria, 1-3 October 2001.

[114] F. Blais, J. A. Beraldin, S. F. El-Hakim, and L. Cournoyer, "Comparison of Pose Estimation Methods of a 3D Laser Tracking System using Triangulation 
and Photogrammetry Techniques," in Proceedings of the SPIE: Electronic Imaging, Videometrics and Optical Methods for 3D Shape Measurement VII, S. F. El-Hakim and A. Gruen, Eds., vol. 4309, no. 1, San-Jose, CA, December 2000, pp. 185-194.

[115] K. J. Gasvik, Optical Metrology. John Wiley and Sons, 1987, pp. 58-63.

[116] G. Ritter and J. Wilson, Handbook of Computer Vision Algorithms in Image Algebra, 2nd ed. CRC Press, 2000, pp. 57-60.

[117] E. Trucco and A. Verri, Introductory Techniques for 3D Computer Vision. Upper Saddle, NJ: Prentice-Hall, Inc., 1998, ch. Dealing with Imaging Noise, pp. 56-62.

[118] W. Pratt, Digital Image Processing, 3rd ed. John Wiley and Sons, 2001, pp. $271-273$.

[119] G. Ritter and J. Wilson, Handbook of Computer Vision Algorithms in Image Algebra, 2nd ed. CRC Press, 2000, pp. 65-68.

[120] A. Burian and P. Kuosmanen, "Tuning the smoothness of the recursive median filter," IEEE Transactions on Signal Processing, vol. 50, no. 7, pp. 1631-1639, 2002 .

[121] A. Restrepo Palacios and L. Chacon, "A smoothing property of the median filter," IEEE Transactions on Signal Processing, vol. 42, no. 6, pp. 1553-1555, 1994.

[122] E. Davies, "A remnant noise problem with the median filter," in Proceedings of the 11th IAPR International Conference on Image, Speech and Signal Analysis in Pattern Recognition, vol. III, 30 Aug-3 Sep 1992, pp. 505-508.

[123] E. Trucco and A. Verri, Introductory Techniques for 3D Computer Vision. Upper Saddle, NJ: Prentice-Hall, Inc., 1998, pp. 62-63. 
[124] I. Pitas and A. Venetsanopoulos, "Edge Detectors Based on Nonlinear Filters," IEEE Transactions on Pattern Analysis and Machine Intelligence, vol. 8, no. 4, pp. 538-550, July 1986.

[125] Y. Zou and W. Dunsmuir, "Generalized max/median filtering," in Proceedings of the International Conference on Image Processing, vol. 1, 26-29 Oct 1997, pp. 428431.

[126] X. Zhou and W. Ye, "A conjecture on iterated three-point median filter," IEEE Transactions on Signal Processing, vol. 44, no. 9, pp. 2347-2350, 1996.

[127] P. Wendt, "Nonrecursive and recursive stack filters and their filtering behavior," IEEE Transactions on Acoustics, Speech, and Signal Processing, vol. 38, no. 12, pp. 2099-2107, 1990.

[128] E. Coyle, M. Gabbouj, and J.-H. Lin, "From median filters to optimal stack filtering," in IEEE International Sympoisum on Circuits and Systems, vol. 1, 11-14 June 1991, pp. 9-12.

[129] G. Qiu, "An improved recursive median filtering scheme for image processing," IEEE Transactions on Image Processing, vol. 5, no. 4, pp. 646-648, 1996.

[130] D. Mittermair and P. Puschner, "Which sorting algorithms to choose for hard realtime applications," in Proceedings of the Ninth Euromicro Workshop on Real-Time Systems, 11-13 Jun 1997, pp. 250-257.

[131] G. Angelopoulos and I. Pitas, "A fast implementation of two-dimensional weighted median filters," in Proceedings of the 12th IAPR International Conference on Signal Processing in Pattern Recognition, vol. 3, 9-13 Oct 1994, pp. 140-142.

[132] W. Mendenhall, Mathematical Statistics with Applictions, 3rd ed. Boston, Massachusetts: PWS Publishers, 1986, ch. 1: What is Statistics?, p. 9. 
[133] D. MacKinnon, V. Aitken, and F. Blais, "Using Quality Metrics with Laser Range Scanners," in Proceedings of the ISTET/SPIE 20th Annual Symposium on Electronic Imaging Science and Technology, San Jose, CA, USA, 27-31 January 2008.

[134] —-, "Adaptive Laser Range Scanning using Quality Metrics," in Proceedings of the IEEE International Instrumentation and Measurement Technology Conference, Victoria, BC, CAN, 12-15 May 2008.

[135] W. Mendenhall, Mathematical Statistics with Applictions, 3rd ed. Boston, Massachusetts: PWS Publishers, 1986, ch. Appendix III: Tables, p. 696.

[136] D. MacKinnon, V. Aitken, F. Blais, and M. Picard, "Adaptive Laser Range Scanning," in Proceedings of the IEEE International Workshop on Robotic and Sensors Environments, Ottawa, ON, Canada, 12-13 October 2007.

[137] D. MacKinnon, V. Aitken, and F. Blais, "Adaptive Laser Range Scanning," in Proceedings of the American Controls Conference, 11-13 June 2008.

[138] W. Mendenhall, Mathematical Statistics with Applictions, 3rd ed. Boston, Massachusetts: PWS Publishers, 1986, ch. Appendix III: Tables, p. 699.

[139] I. Stamos and P. K. Allen, "3-D Model Construction Using Range and Image Data," in Proceedings of the IEEE Conference on Computer Vision and Pattern Recognition, vol. 1, Hilton Head Island, SC, USA, 13-15 June 2000, pp. 531-536.

[140] R. Jain, R. Kasturi, and B. G. Schunck, Machine Vision. McGraw-Hill, Inc., 1995, ch. Steps in Edge Detection, pp. 147-148.

[141] J. Chen, T. Leung, H.and Lo, J. Litva, and M. Blanchette, "A modified probabilistic data association filter in a real clutter environment," IEEE Transactions on Aerospace and Electronic Systems, vol. 32, no. 1, pp. 300-313, January 1996. 
[142] L. Perera, W. Wijesoma, and M. Adams, "Data Association in Dynamic Environments Using a Sliding Window of Temporal Measurement Frames," in Proceedings of the IEEE/RSJ International Conference on Intelligent Robots and Systems. IEEE, 2-6 August 2005, pp. 753- 758.

[143] J. Andrade-Cetto and A. Sanfeliu, "The Effects of Partial Observability When Building Fully Correlated Maps," IEEE Transactions on Robotics, vol. 21, no. 4, pp. 771-777, August 2005.

[144] M. Farooq, A. Rouhi, and D. Horsman, "Analysis of Suboptimal Kalman Tracking Algorithms," in Proceedings of the 28th Conference on Decision and Control, Tampa, FL, USA, 1989, pp. 1417-1422.

[145] S. Cong, L. Hong, and D. Wicker, "Markov-chain Monte-Carlo approach for association probability evaluation," IEEE Process-Control Theory and Applications, vol. 151, no. 2, pp. 185-193, March 2004.

[146] I. J. Cox, "A Review of Statistical Data Association Techniques for Motion Correspondence," International Journal of Computer Vision, vol. 10, no. 1, pp. 53-66, 1993.

[147] A. Rouhi, M. Farooq, and S. Bruder, "Multiple structure adaptive target tracking," in Proceedings of the 32nd Midwest Symposium on Circuits and Systems, vol. 1, 1416 August 1990, pp. $613-616$.

[148] X. R. Li, "Canonical Transform for Tracking with Kinematic Models," IEEE Transactions on Aerospace and Electronic Systems, vol. 33, no. 4, pp. 1212-1224, October 1997. 
[149] M. Farooq and S. Bruder, "Information Type Filters for Tracking a Maneuvering Target," IEEE Transactions on Aerospace and Electronic Systems, vol. 26, no. 3, pp. 441-454, May 1990.

[150] R. Baheti and D. O'Hallaron, "Efficient parallel implementation of target tracking Kalman filter," in Proceedings of the 27th IEEE Conference on Decision and Control, ser. 7-9 December, vol. 1, Austin, TX, USA, 1988, pp. 376-381.

[151] P. S. Maybeck, Stochastic models, Estimation and Control. Academic Press, 1979, vol. 1, ch. 1: Introduction, pp. 1-16.

[152] P. Hebert and M. Rioux, "Toward a handheld laser range scanner: integrating observation-based motion compensation," in Proceedings of the SPIE: ThreeDimensional Image Capture and Applications, R. N. Ellson and J. H. Nurre, Eds., vol. 3313, no. 1, San Jose, California, 27-28 January 1998, pp. 2-13.

[153] F. Blais, M. Picard, and G. Godin, "Accurate 3D Acquisition of Freely Moving Objects," in Proceedings of the Second International Symposium on $3 D$ Data Processing, Visualization and Transmission, Thessaloniki, Greece, 6-9 September 2004, pp. 422-429.

[154] D. Simon, M. Hebert, and T. Kanade, "Real-time 3-D pose estimation using a high-speed range sensor," in Proceedings of the IEEE International Conference on Robotics and Automation, vol. 3, San Diego, CA, USA, 8-13 May 1994, pp. 22352241.

[155] P. Hebert, "A Self-Referenced Hand-Held Range Sensor," in Proceedings of the Third International Conference on 3D Imaging and Modeling, Quebec City, QC, Canada, 28 May-1 June 2001, pp. 5-12. 
[156] K. Nishino and K. Ikeuchi, "Robust Simultaneous Registration of Multiple Range Images," in Proceedings of the 5th Asian Conference on Computer Vision, Melbourne, Australia, 2325 January 2002, pp. 454-461.

[157] D. Robinson and P. Milanfar, "Fundamental performance limits in image registration," IEEE Transactions on Image Processing, vol. 13, no. 9, pp. 1185-1199, 2004.

[158] X. Pennec and J.-P. Thirion, "A Framework for Uncertainty and Validation of 3D Registration Methods Based on Points and Frames," International Journal of Computer Vision, vol. 25, no. 3, pp. 203 - 229, December 1997.

[159] C. Witzgall and G. S. Cheok, "Registering 3D Point Clouds: An Experimental Evaluation," National Institute of Standards and Technology, Gaithersburg, MD, USA, Technical Report NISTIR 6743, May 2001.

[160] Y. Chen and G. Medioni, "Object modelling by registration of multiple range images," Image and Vision Computing, vol. 10, no. 3, pp. 145-155, April 1992.

[161] H. Gagnon, M. Soucy, R. Bergevin, and D. Laurendeau, "Registration of multiple range views for automatic $3-\mathrm{D}$ model building," in Proceedings of the IEEE Computer Society Conference onComputer Vision and Pattern Recognition, Seattle, Washington, USA, 21-23 June 1994, pp. 581-586.

[162] R. Yang and P. Allen, "Registering, integrating, and building CAD models from range data," in Proceedings of the IEEE International Conference on Robotics and Automation, vol. 4, 16-20 May 1998, pp. 3115-3120.

[163] C. Dorai, G. Wang, C. Mercer, and A. K. Jain, "From Images to Models: Automatic 3D Object Model Construction from Multiple Views," in Proceedings of the IEEE 
International Conference on Pattern Recognition, vol. I, Washington, DC, USA, 1996, pp. $770-774$.

[164] T. Masuda, "Object shape modelling from multiple range images by matching signed distance fields," in Proceedings of the First International Symposium on $3 D$ Data Processing Visualization and Transmission, 2002, pp. 439-448.

[165] F. Remondino, A. Guarnieri, and A. Vettore, "3D Photogrammetry of CloseRange Objects: Photogrammetry of Laser Scanning," in Proceedings of the SPIE: Videometrics VIII, J.-A. Beraldin, S. F. El-Hakim, A. Gruen, and J. Walton, Eds., vol. 5665, San Jose, California, USA, 18-20 January 2005, pp. 216-225.

[166] S. El-Hakim, J. Fryer, M. Picard, and E. Whitting, "Digital recording of aboriginal rock art," in Proceedings of the 10th International Conference on Virtual Systems and Multimedia, Ogaki City, Gifu, Japan, 17-19 November 2004, pp. 344-353.

[167] Z.-W. Zheng and Y.-S. Zhu, "New least squares registration algorithm for data fusion," IEEE Transactions on Aerospace and Electronic Systems, vol. 40, no. 4, pp. $1410-1416$, October 2004.

[168] G. Soucy, F. G. Callari, and F. P. Ferrie, "Estimating pose through local geometry," in Proceedings of the Second International Conference on 3D Imaging and Modeling, Ottawa, ON, Can, 4-8 October 1999, pp. 220-229.

[169] J. Fleischer, S. Marsland, and J. Shapiro, "Sensory Anticipation for Autonomous Selection of Robot Landmarks," in Anticipatory Behavior in Adaptive Learning Systems: Foundations, Theories, and Systems, ser. Lecture Notes in Computer Science, M. V. Butz, O. Sigaud, and P. Gérard, Eds. Springer Berlin / Heidelberg, January 2003, vol. 2684, pp. 201-221. 
[170] S. Thrun, "Robotic Mapping: A Survey," in Exploring Artificial Intelligence in the New Millennium, G. Lakemeyer and B. Nevel, Eds. Morgan Kaufmann, 2002.

[171] C. Brenneke, O. Wuif, and B. Wagner, "Using 3D Laser Range Data for SLAM in Outdoor Environments," in Proceedings of the IEEE/RSJ International Conference on Intelligent Robots and Systems, Las Vegas, Nevada, USA, October 2003, pp. 188-193.

[172] I. Stamos and M. Leordeanu, "Automated feature-based range registration of urban scenes of large scale," in Proceedings of the IEEE Computer Society Conference on Computer Vision and Pattern Recognition, vol. 2, 18-20 June 2003, pp. II-555-II561.

[173] A. B. Abche, G. S. Tzanakos, and E. Micheli-Tzanakou, "A Method For Multimodal 3-D Image Registration with External Markers," in Proceedings of the Annual International Conference of the IEEE Engineering in Medicine and Biology Society, vol. 14, 29 October-1 November 1992, pp. 1881-1882.

[174] M. V. Butz, S. Swarup, and D. E. Goldberg, "Effective Online Detection of TaskIndependent Landmarks," Illinois Genetic Algorithms Laboratory, University of Illinois at Urbana-Champaign, Urbana, IL, USA, Tech. Rep. 2004002, January 2004.

[175] W. Boehler, M. B. Vincent, and A. Marbs, "Investigating Laser Range Scanner Accuracy," in Proceedings of the XIXth CIPA Symposium, Antalya, Turkey, 30 September - 4 October 2004.

[176] K. Klein and V. Sequeira, "View planning for the 3D modelling of real world scenes," in Proceedings of the IEEE/RSJ International Conference on Intelligent Robots and Systems, vol. 2, 31 October-5 November 2000, pp. 943-948. 
[177] W. Scott, G. Roth, and J.-F. Rivest, "Performance-oriented view planning for automatic model acquisition," in Proceedings of the 31st International Symposium on Robotics, Montreal, QC, Canada, 2000, pp. 314-319.

[178] - "View Planning with a Registration Component," in Proceedings of the 3D Imaging and Modeling Conference, Québec City, QC, Canada, May 28 - June 1 2001, pp. 127-134.

[179] ——, "View Planning with Positioning System Error," National Research Council of Canada, Tech. Rep. NRC/ERB-1086, May 2001.

[180] —, "View Planning as a Set Covering Problem," National Research Council of Canada, Tech. Rep. NRC/ERB-1089, August 2001.

[181] W. Scott, G. Roth, and J. Rivest, "View planning with a registration constraint," in Proceedings of the Third International IEEE Conference on 3-D Digital Imaging and Modeling, Quebec City, QC, Canada, 28 May - 1 June 2001, pp. 127-134.

[182] R. A. Pito, "Automated surface acquisition using range cameras," PhD Thesis, University of Pennsylvania, 1997.

[183] R. Pito, "A solution to the next best view problem for automated surface acquisition," IEEE Transactions on Pattern Analysis and Machine Intelligence, vol. 21 , no. 10 , pp. 1016-1030, October 1999.

[184] D. Papadopoulos-Orfanos and F. Schmitt, "Automatic 3-D digitization using a laser rangefinder with a small field of view," in Proceedings of the International Conference on Recent Advances in 3-D Digital Imaging and Modeling, Ottawa, ON, Canada, 12-15 May 1997, pp. 60-67.

[185] P. Allen, I. Stamos, A. Gueorguiev, E. Gold, and P. Blaer, "AVENUE: Automated site modeling in urban environments," in Proceedings of the Third International 
Conference on 3-D Digital Imaging and Modeling, Québec City, QC, Canada, 28 May-1 June 2001, pp. 357-364.

[186] J. Banta, L. Wong, C. Dumont, and M. Abidi, "A next-best-view system for autonomous 3-D object reconstruction," IEEE Transactions on Systems, Man and Cybernetics, Part A, vol. 30, no. 5, pp. 589-598, September 2000.

[187] Y. Li, B. He, and P. Bao, "Automatic view planning with self-termination in 3D object reconstructions," Sensors and Actuators A: Physical, vol. 122, no. 2, pp. 335-344, August 2005.

[188] Y. Li, B. He, S. Chen, and P. Bao, "A view planning method incorporating self-termination for automated surface measurement," Measurement Science and Technology, vol. 16, no. 9, pp. 1865-1877, September 2005.

[189] Y. Liu, "Multi-scale 3-D map building and representation for robotics applications and vehicle guidance," PhD Thesis, Université Laval, 1997.

[190] J. Maver and R. Bajcsy, "Occlusions as a guide for planning the next view," IEEE Transactions on Pattern Analysis and Machine Intelligence, vol. 15, no. 5, pp. 417-433, May 1993.

[191] M. Reed, P. Allen, and I. Stamos, "Automated model acquisition from range images with view planning," in Proceedings of the IEEE Computer Society Conference on Computer Vision and Pattern Recognition, 17-19 June 1997, pp. 72-77.

[192] S. Roy, S. Chaudhury, and S. Banerjee, "Recognizing large isolated 3-D objects through next view planning using inner camera invariants," IEEE Transactions on Systems, Man and Cybernetics, Part B, vol. 35, no. 2, pp. 282-292, April 2005.

[193] F. Zhao, J. Shin, and J. Reich, "Information-driven dynamic sensor collaboration," IEEE Signal Processing Magazine, vol. 19, no. 2, pp. 61-72, March 2002. 
[194] R. De Maesschalck, D. Jouan-Rimbaud, and D. L. Massart, "The Mahalanobis distance," Chemometrics and Intelligent Laboratory Systems, vol. 50, no. 1, pp. $1-18$, January 2000.

[195] C. Archambeau and M. Verleysen, "Fully Nonparametric Probability Density Function Estimation with Finite Gaussian Mixture Models," in Proceedings of the 5th International Conference on Advances in Pattern Recognition, Calcutta, India, 13 December 2003, pp. 81-84.

[196] J. Neira and J. D. Tardós, "Data Association in Stochastic Mapping Using the Joint Compatibility Test," IEEE Transactions on Robotics and Automation, vol. 17, no. 6, pp. 890-897, December 2001. 


\section{Appendix A: Calibration}
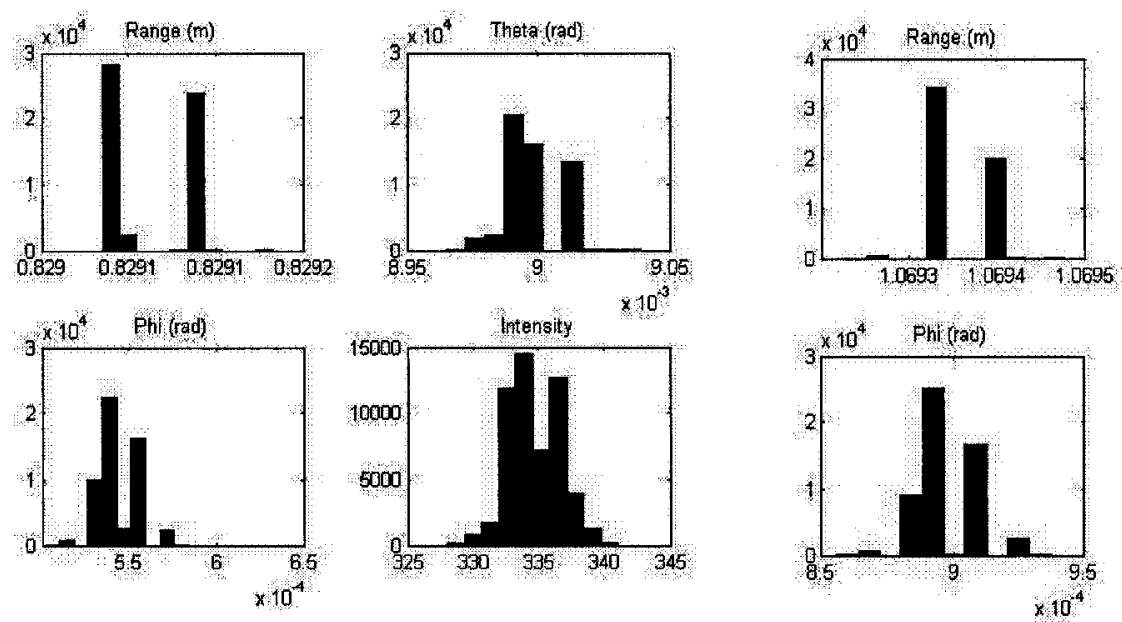

Figure A-1: Data set \#1
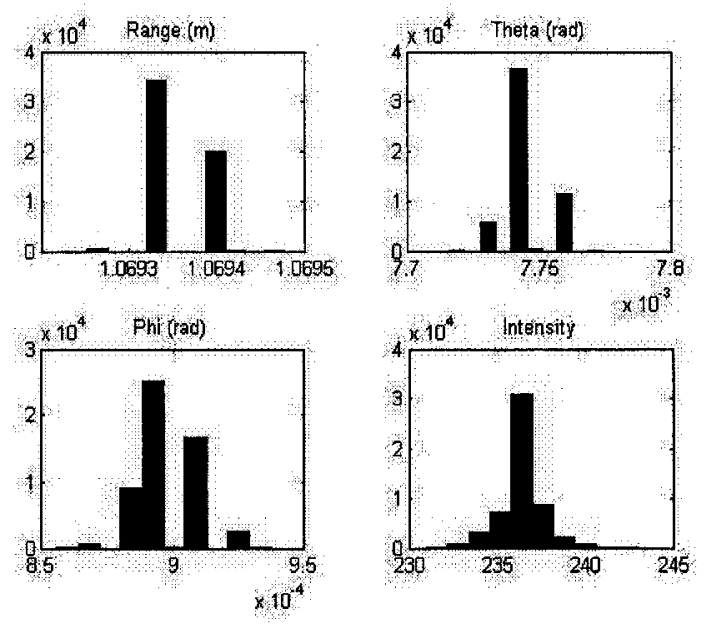

Figure A-2: Data set \#2 

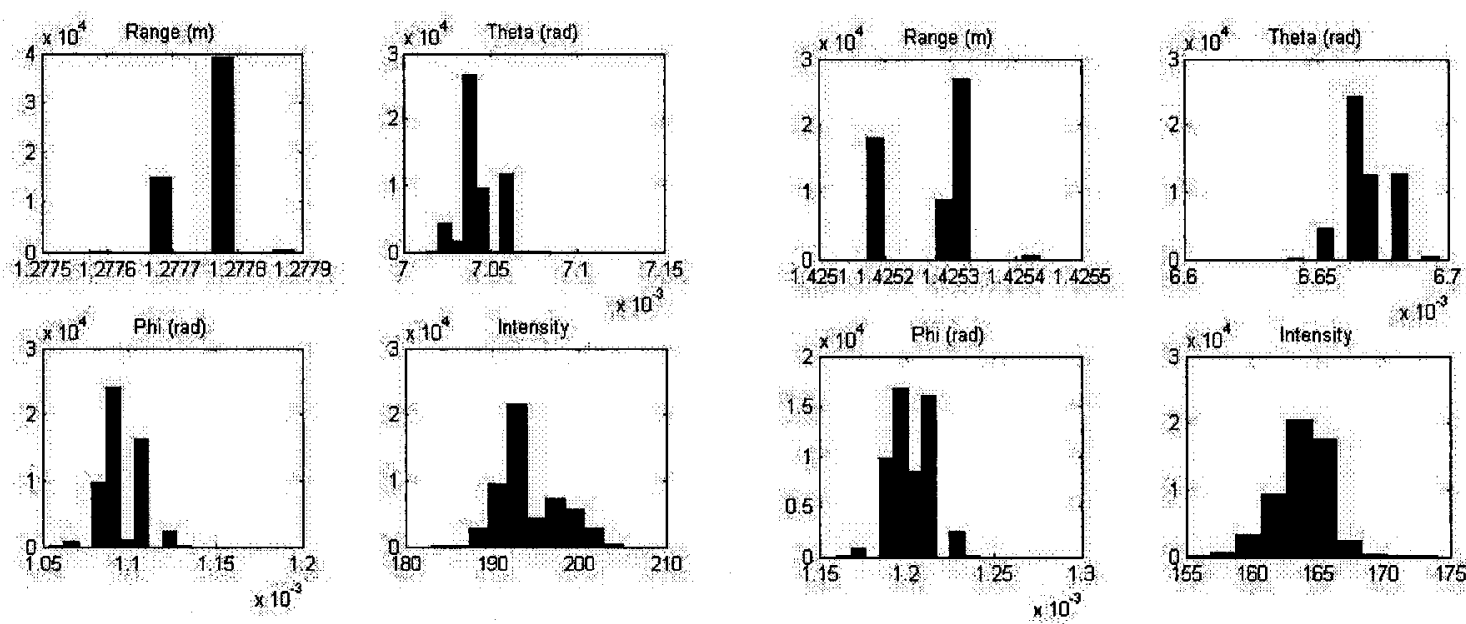

Figure A-3: Data set \#3
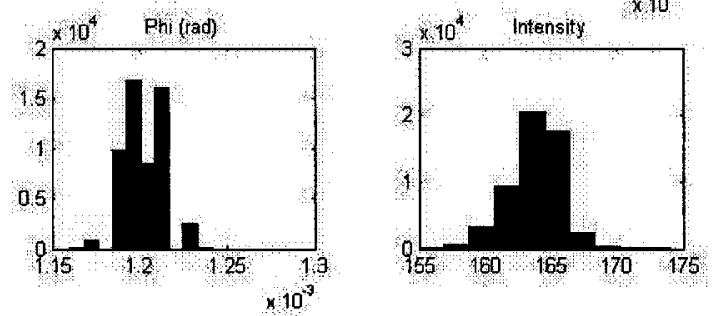

Figure A-4: Data set \#4
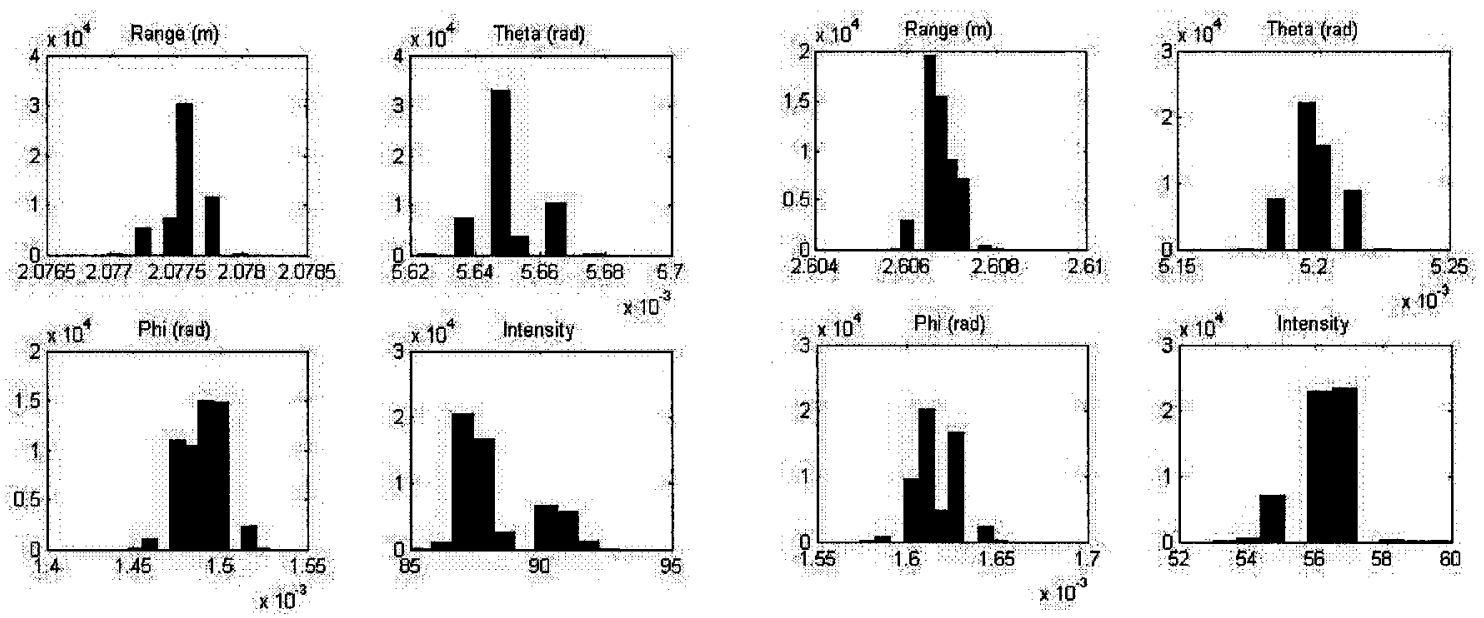

Figure A-5: Data set \#5

Figure A-6: Data set \#6 

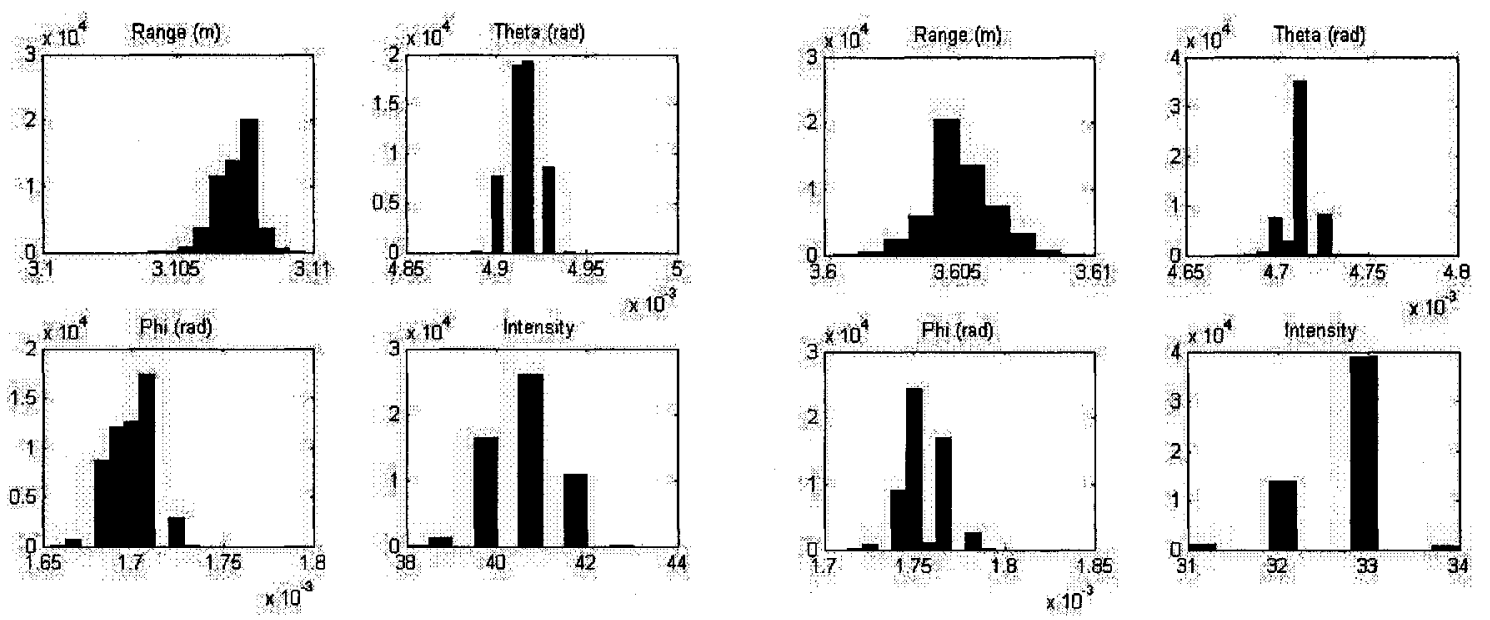

Figure A-7: Data set \#7

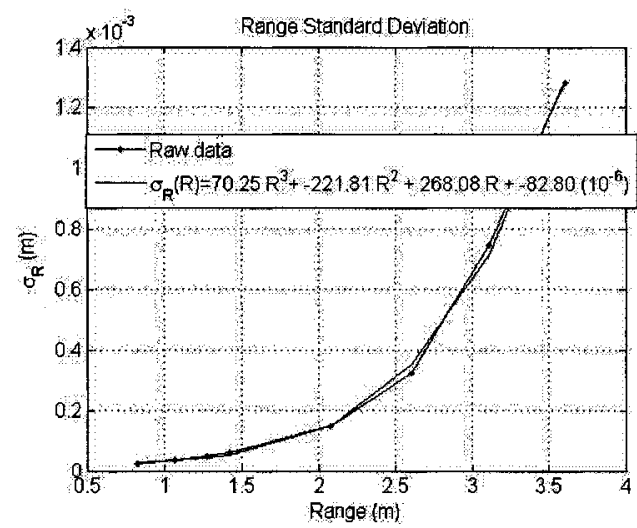

Figure A-9: Range uncertainty model

Figure A-8: Data set \#8

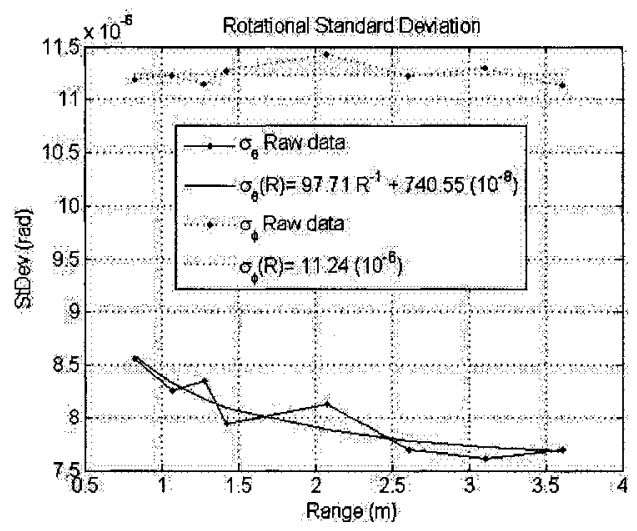

Figure A-10: Rotational uncertainty model 


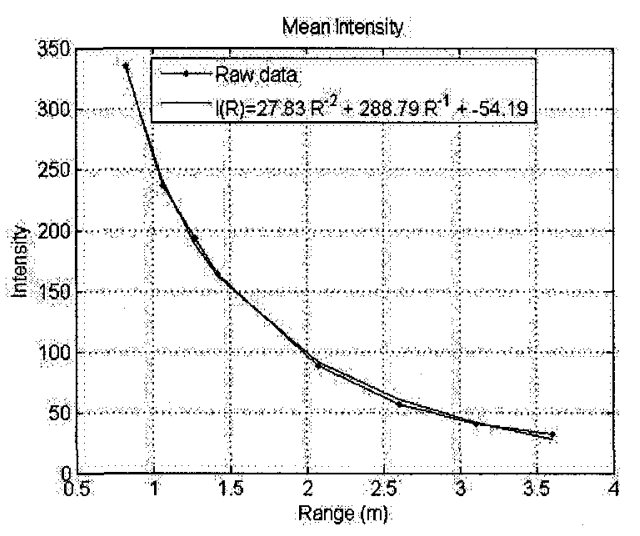

Figure A-11: Reference intensity model

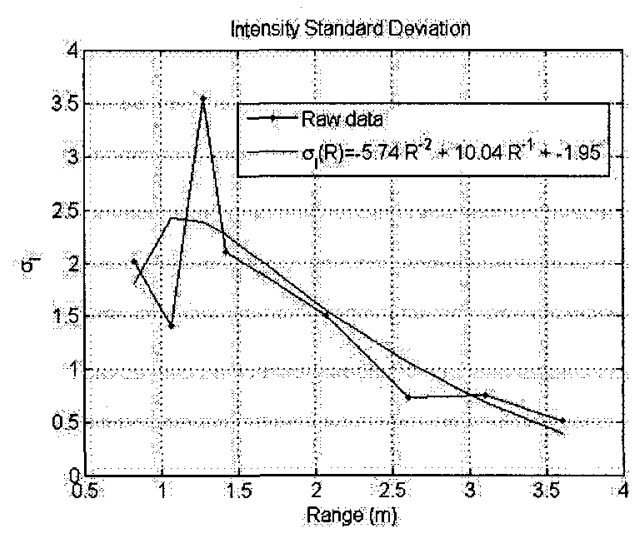

Figure A-12: Intensity uncertainty model 


\section{Appendix B: Test Surfaces}

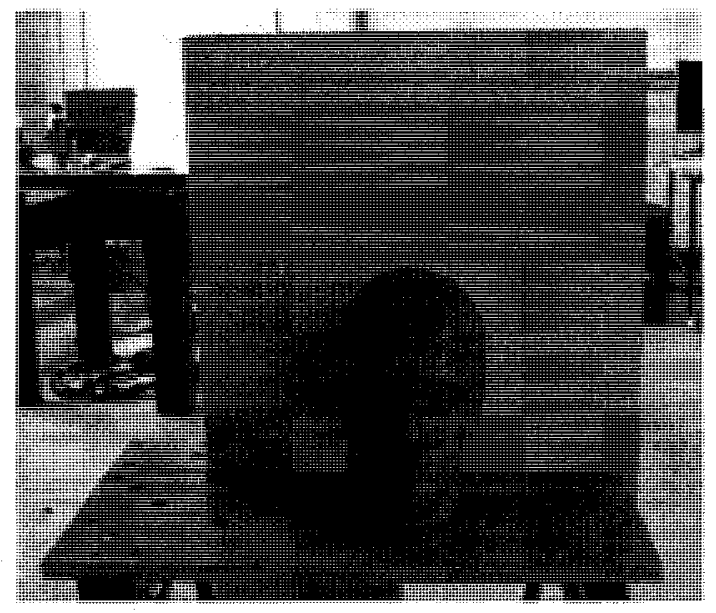

Figure B-1: Experiment \#1: front view

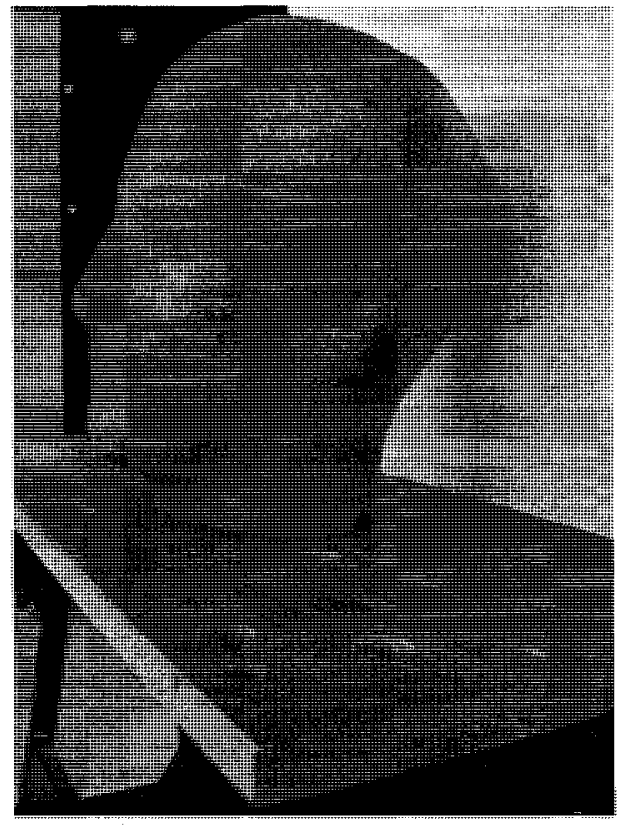

Figure B-2: Experiment \#1: side view 


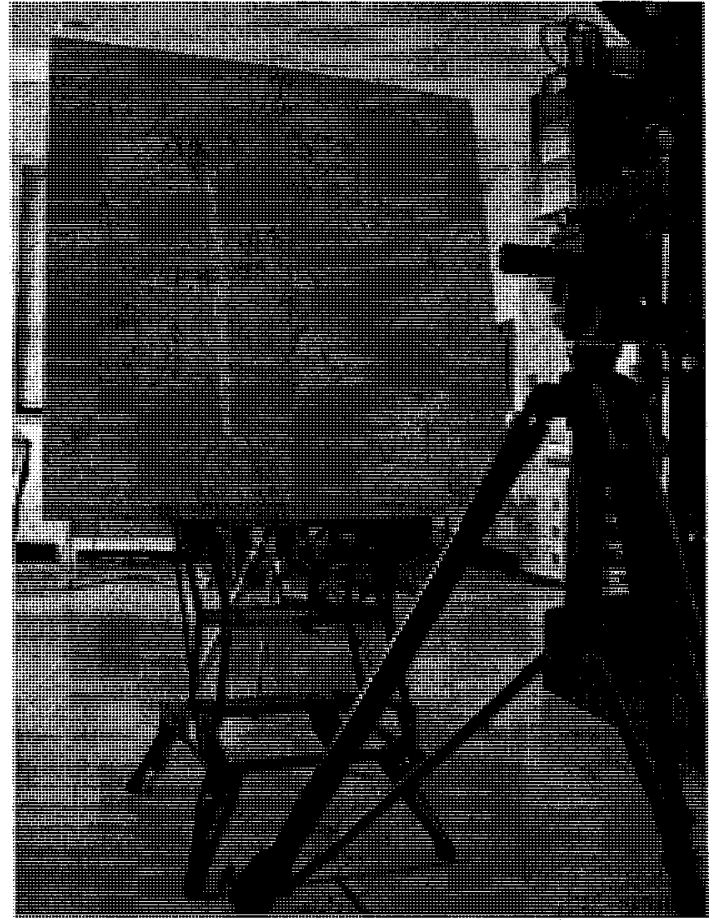

Figure B-3: Experiment \#2: front view

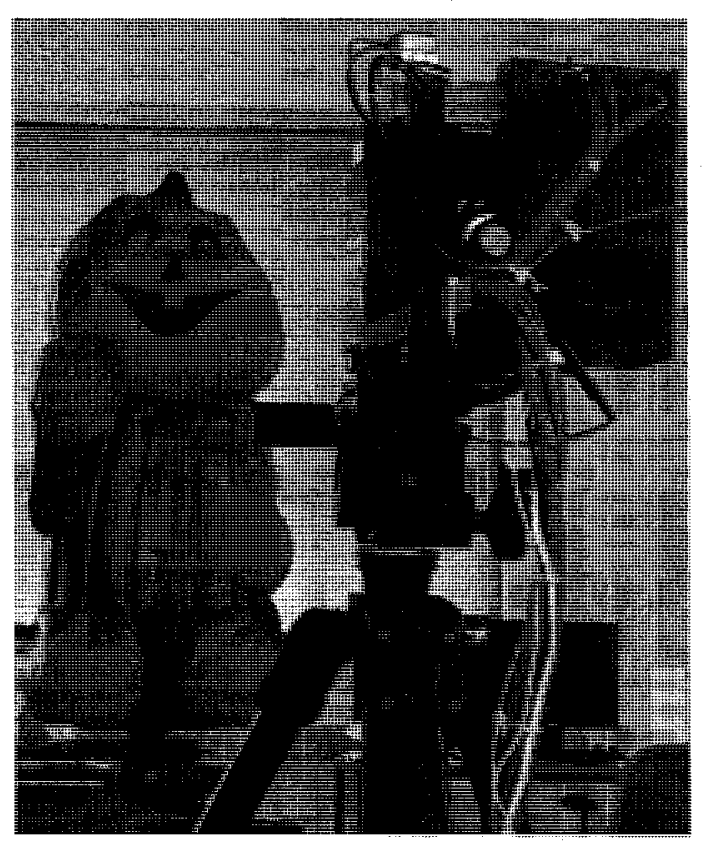

Figure B-5: Experiment \#3: front view

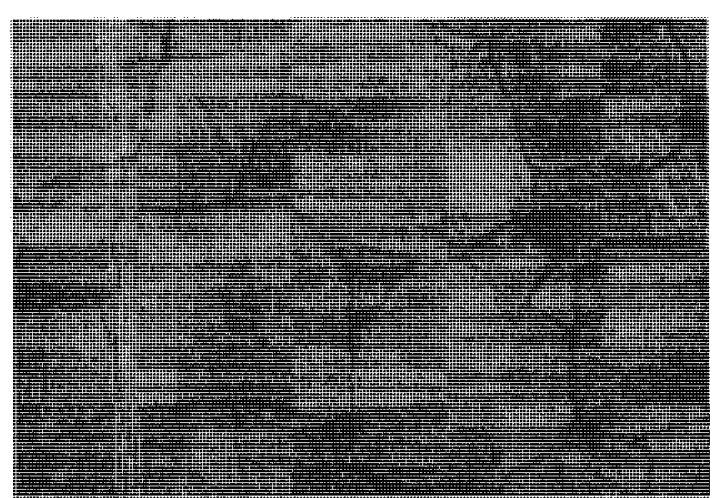

Figure B-4: Experiment \#2: detail view

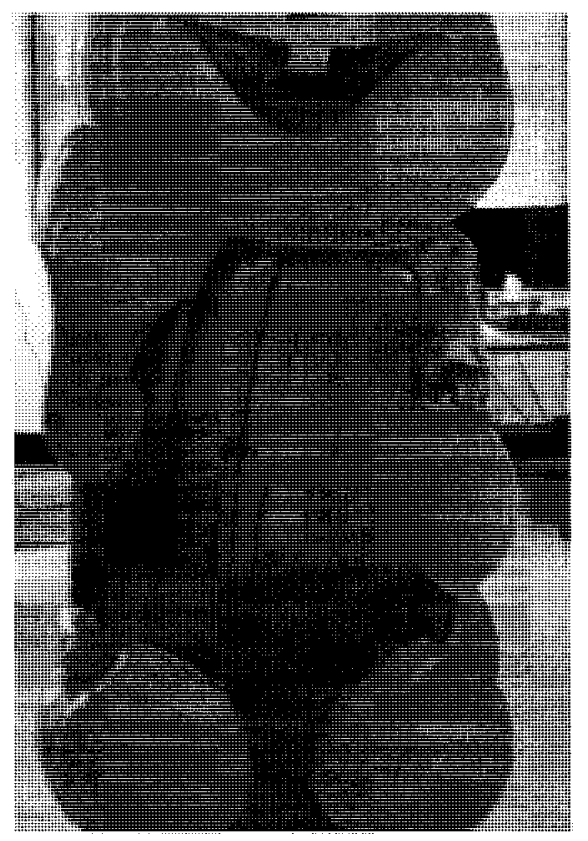

Figure B-6: Experiment \#3: detail view 


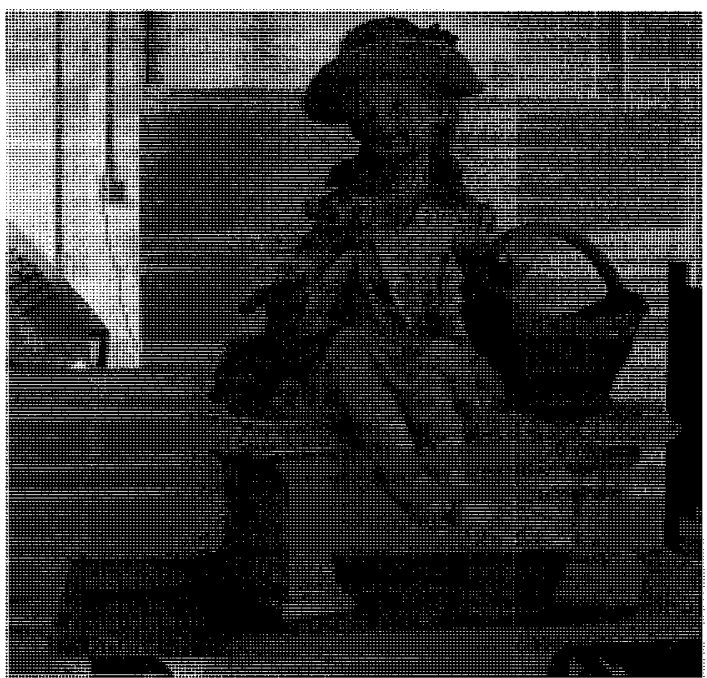

Figure B-7: Experiment \#4: front view

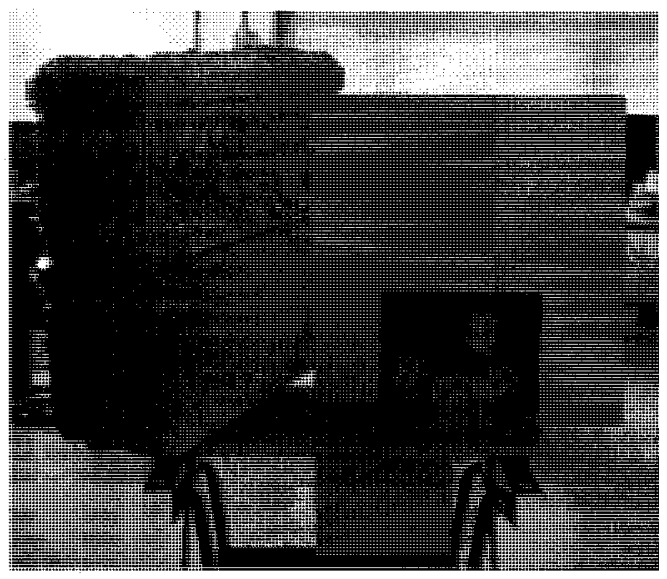

Figure B-9: Experiment \#5: front view

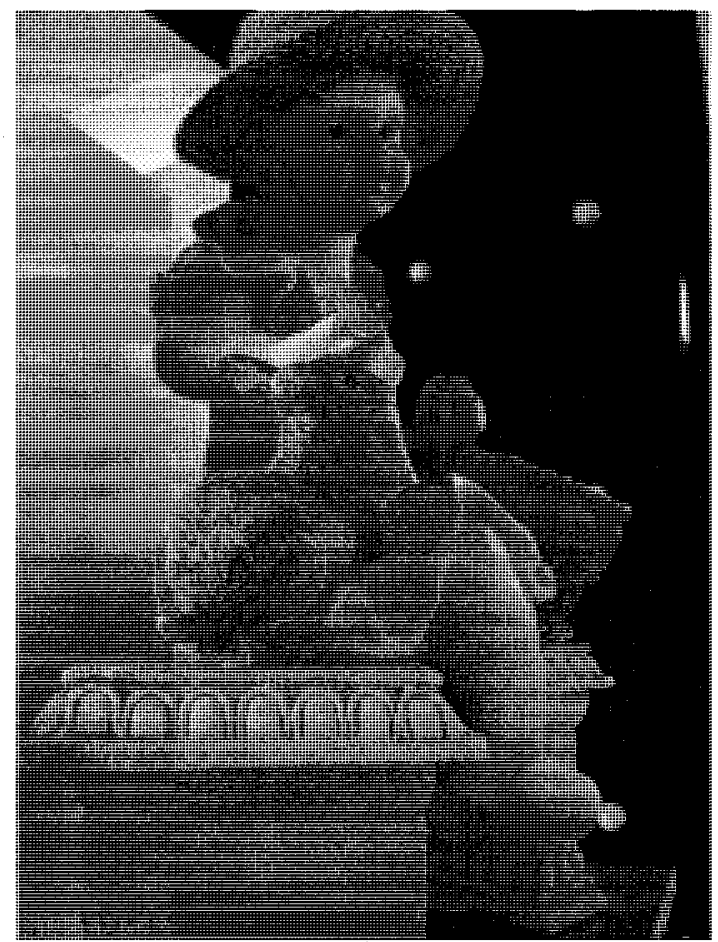

Figure B-8: Experiment \#4: side view

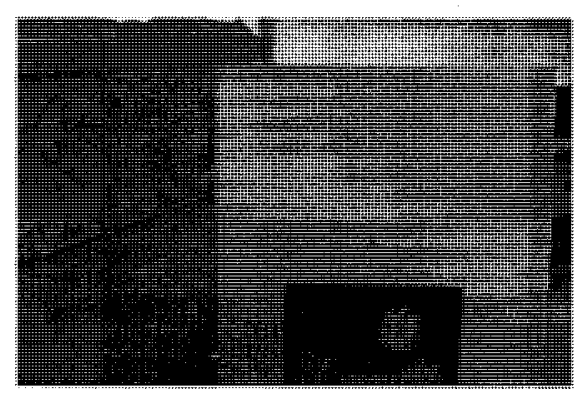

Figure B-10: Experiment \#5: detail view 


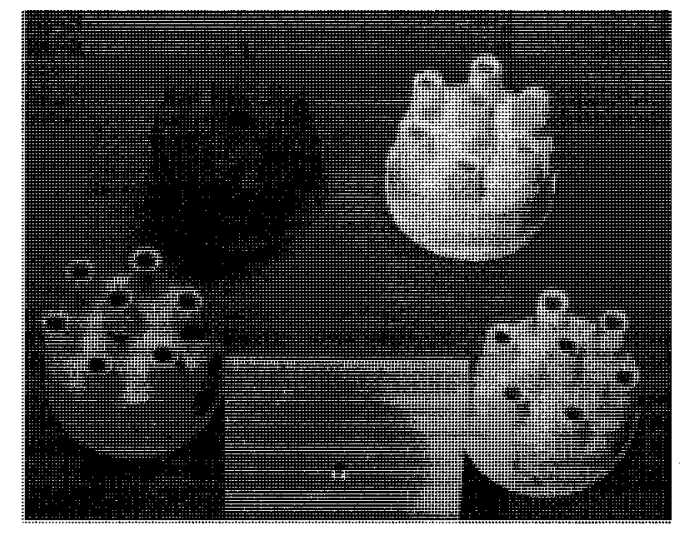

Figure B-11: Experiment \#5: picture detail

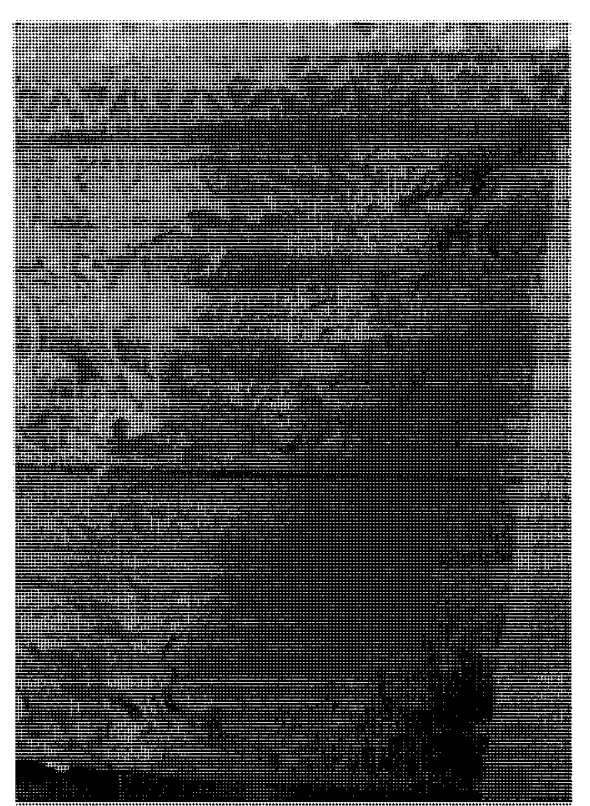

Figure B-12: Experiment \#5: planter detail 


\section{Appendix C: Filter Calibration}

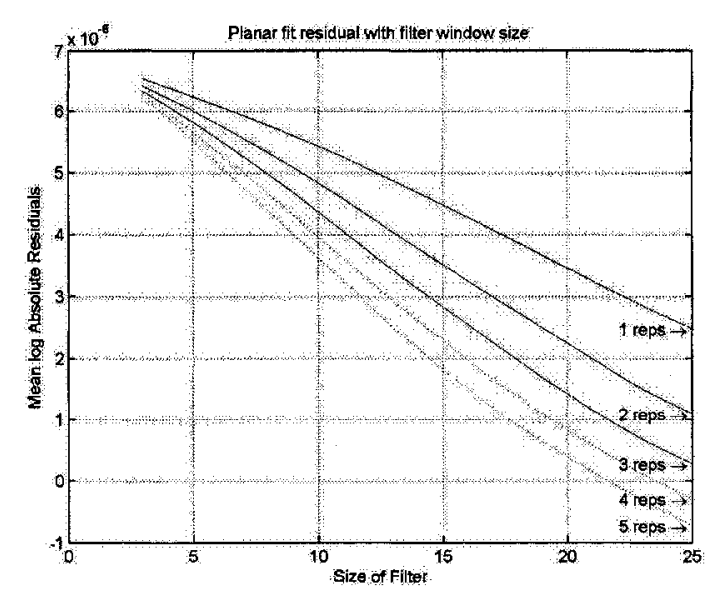

Figure C-1: Scan Area/Spot Size $=0.003$

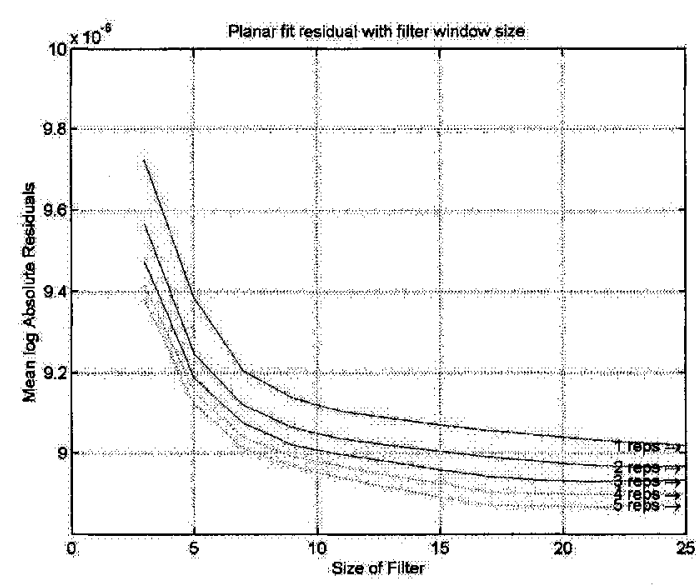

Figure C-2: Scan Area/Spot Size $=0.068$ 


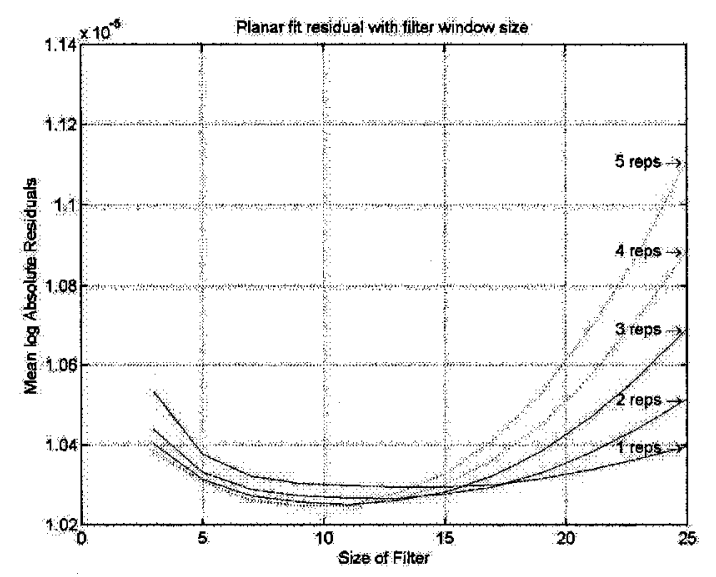

Figure C-3: Scan Area/Spot Size $=0.279$

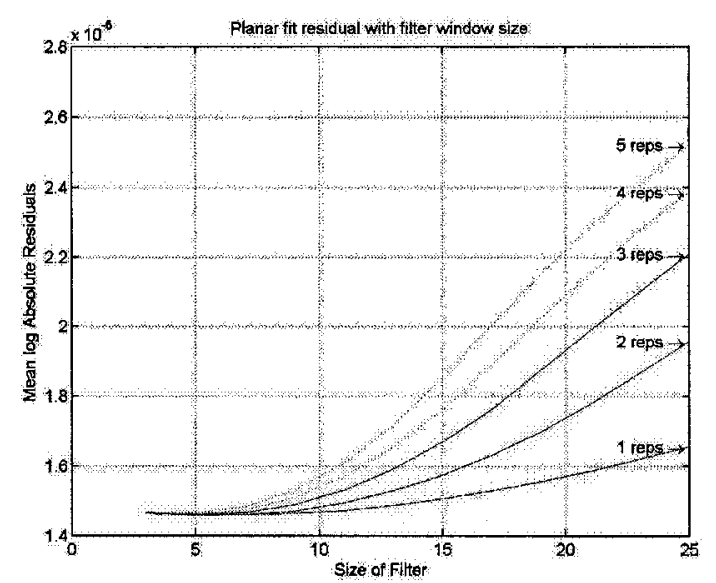

Figure C-5: Scan Area/Spot Size $=2.582$

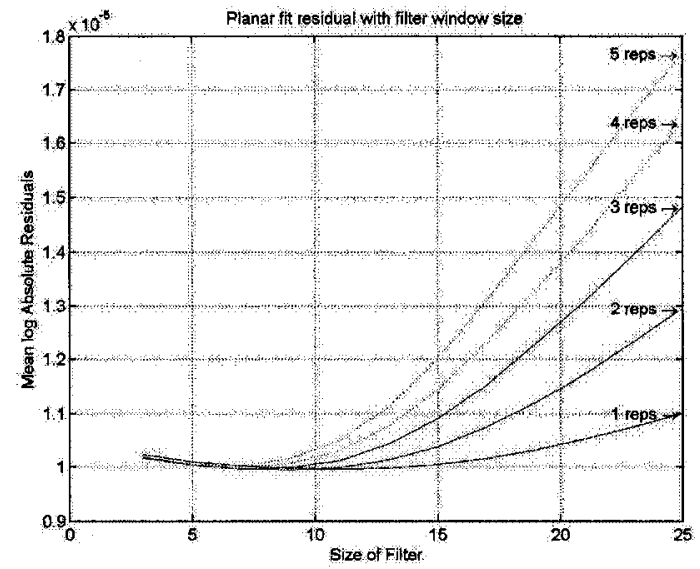

Figure C-4: Scan Area/Spot Size $=1.130$

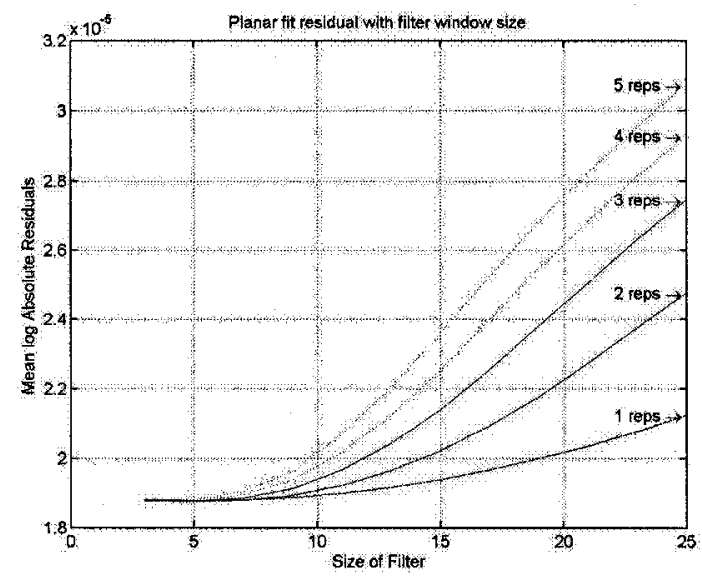

Figure C-6: Scan Area/Spot Size=4.702 

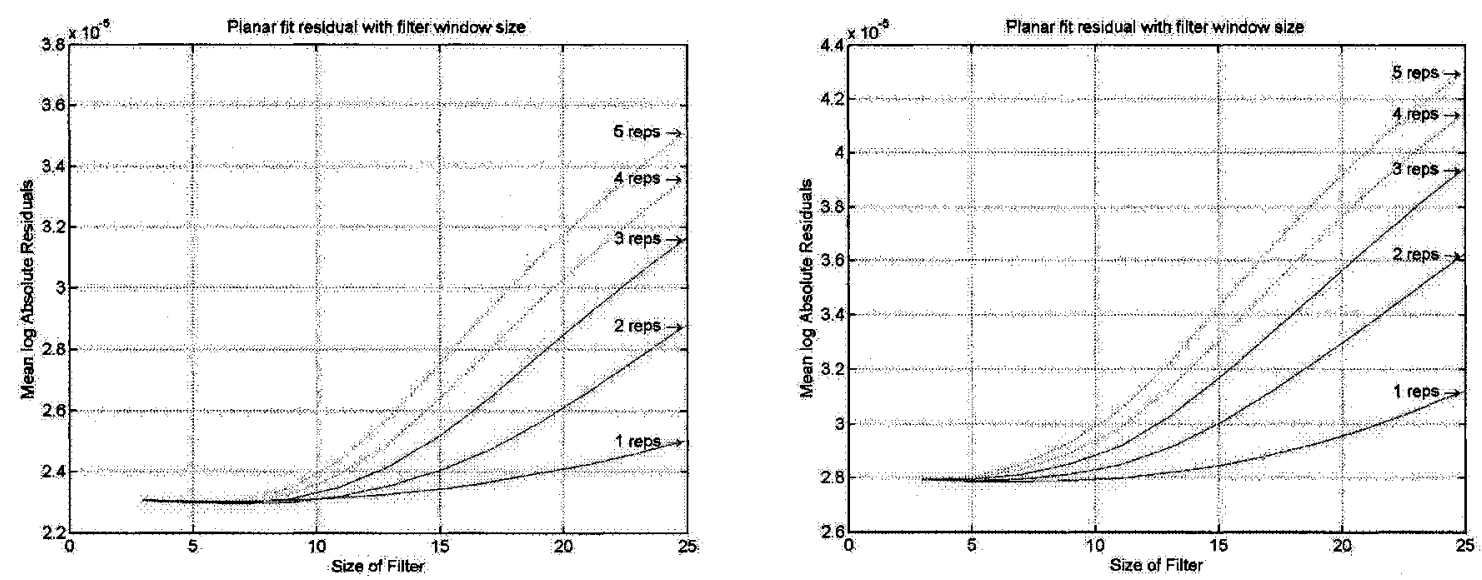

Figure C-7: Scan Area/Spot Size=7.585

Figure C-8: Scan Area/Spot Size $=11.654$ 


\section{Appendix D: Outlier Metric}

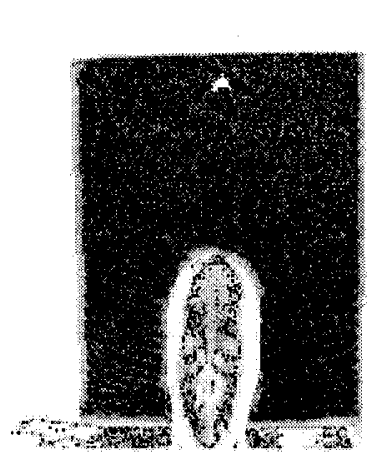

Figure D-1: Experiment \#1 outlier quality map

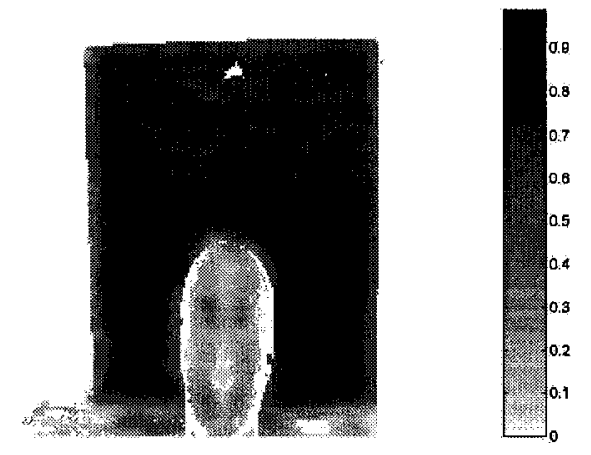

Figure D-2: Experiment \#1 smoothed outlier map 


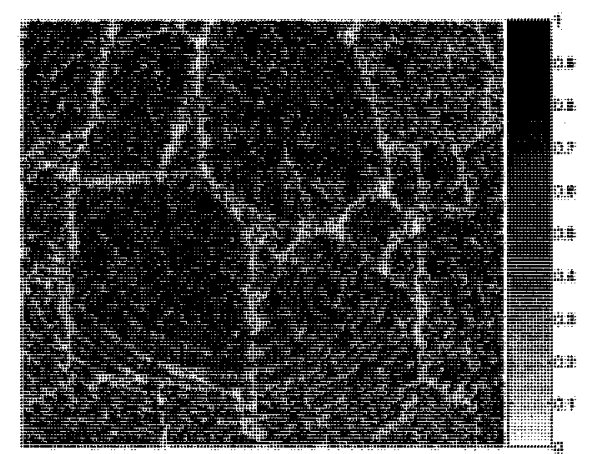

Figure D-3: Experiment \#2 outlier quality map
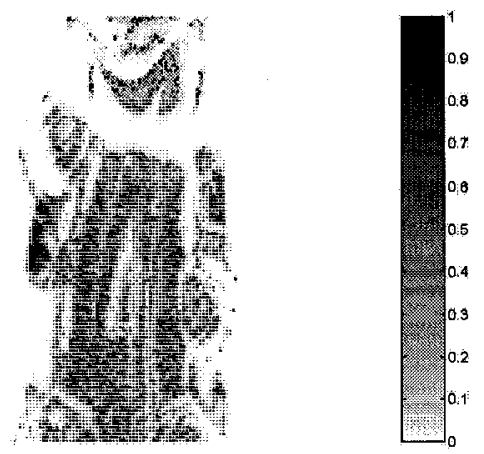

Figure D-5: Experiment \#3 outlier quality map

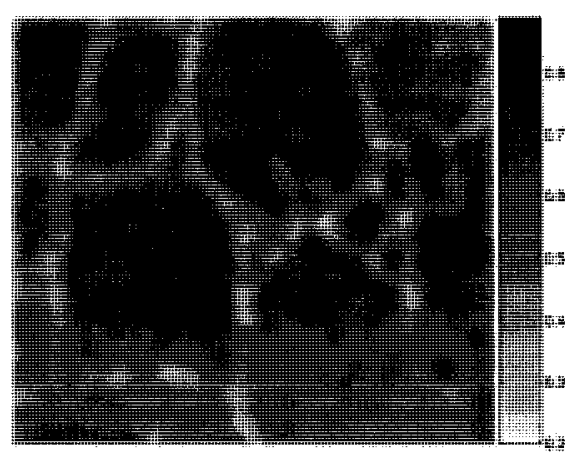

Figure D-4: Experiment \#2 smoothed outlier map
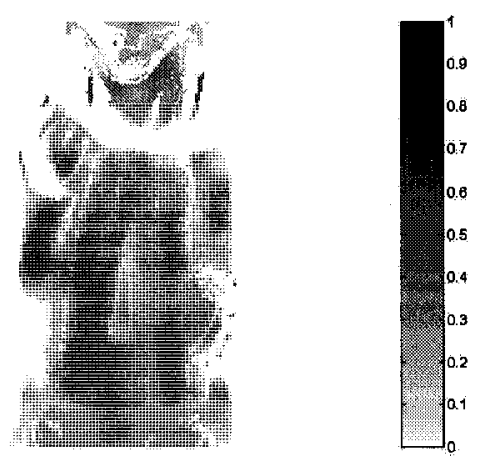

Figure D-6: Experiment \#3 smoothed outlier map 

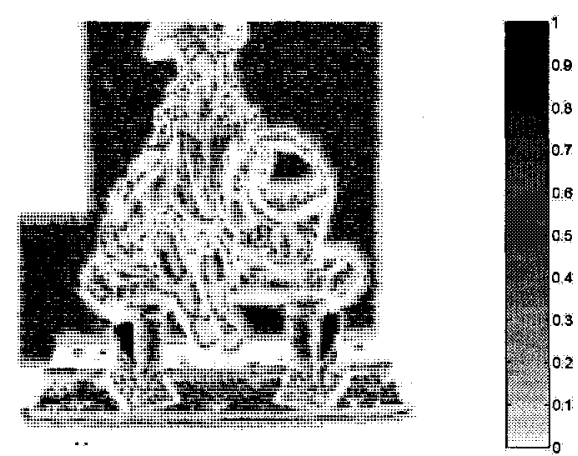

Figure D-7: Experiment \#4 outlier quality map

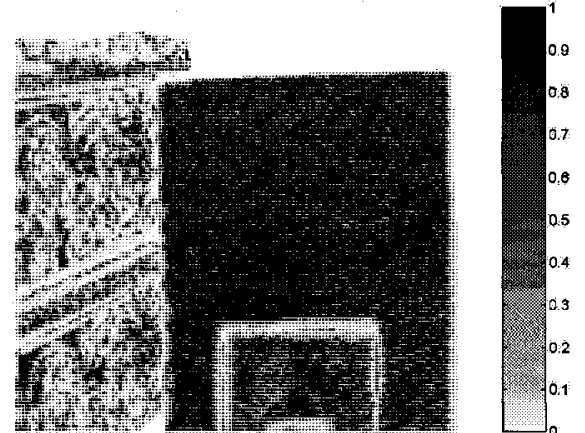

Figure D-9: Experiment \#5 outlier quality map

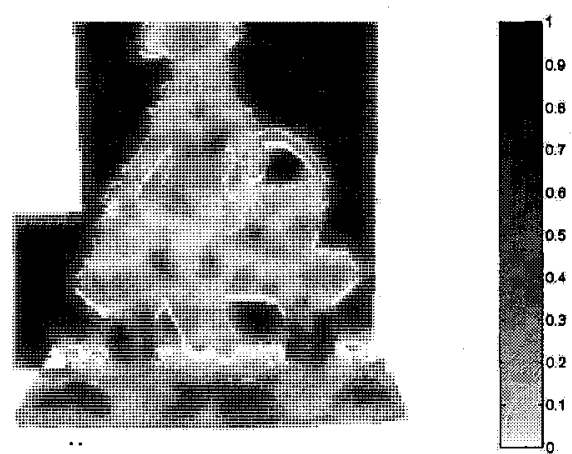

Figure D-8: Experiment \#4 smoothed outlier map

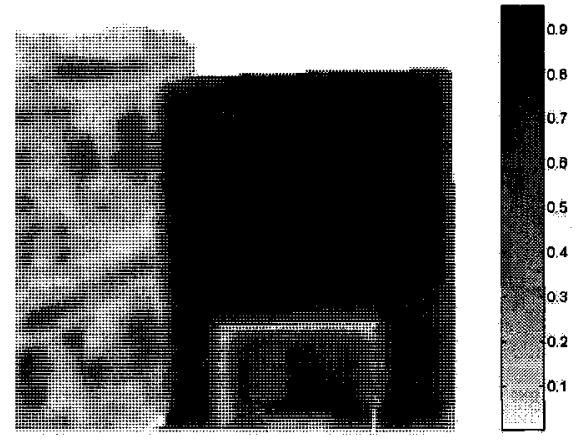

Figure D-10: Experiment \#5 smoothed outlier map 


\section{Appendix E: Orientation and Resolvability Metrics}

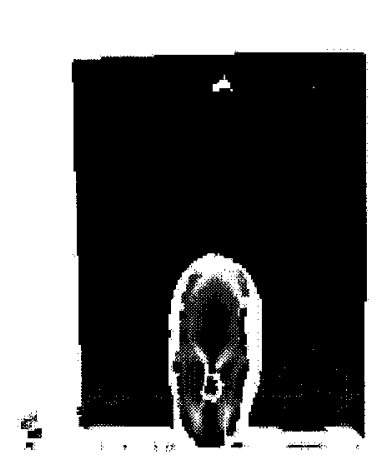

Figure E-1: Experiment \#1 orientation quality map

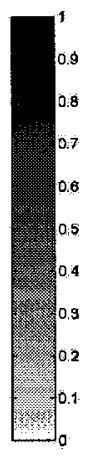

Figure E-2: Experiment \#1 resolvability quality map 


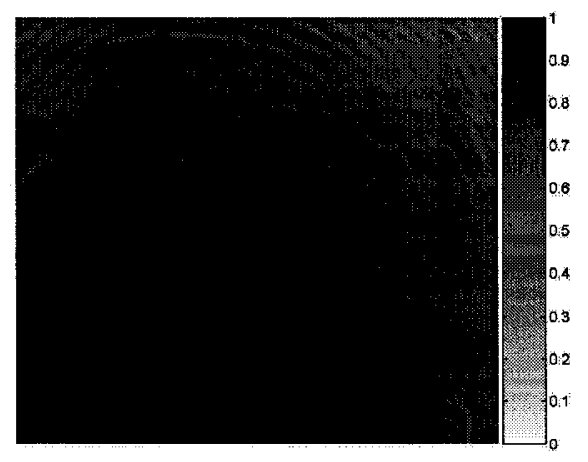

Figure E-3: Experiment \#2 orientation quality map
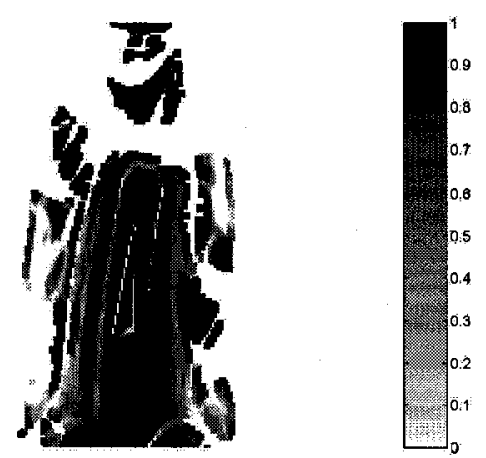

Figure E-5: Experiment \#3 orientation quality map

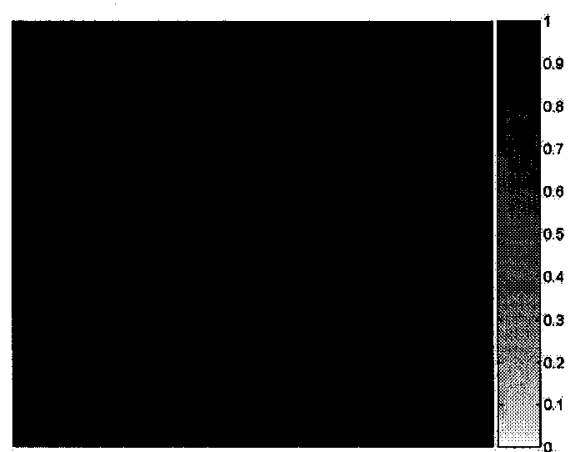

Figure E-4: Experiment \#2 resolvability quality map
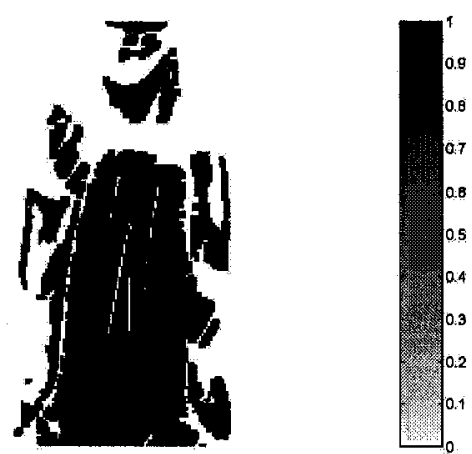

Figure E-6: Experiment \#3 resolvability quality map 

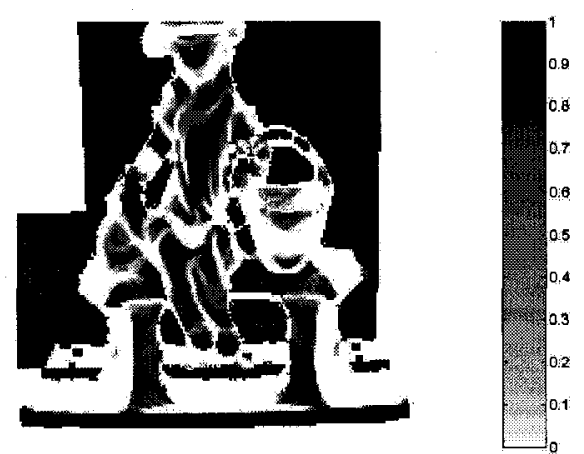

Figure E-7: Experiment \#4 orientation quality map
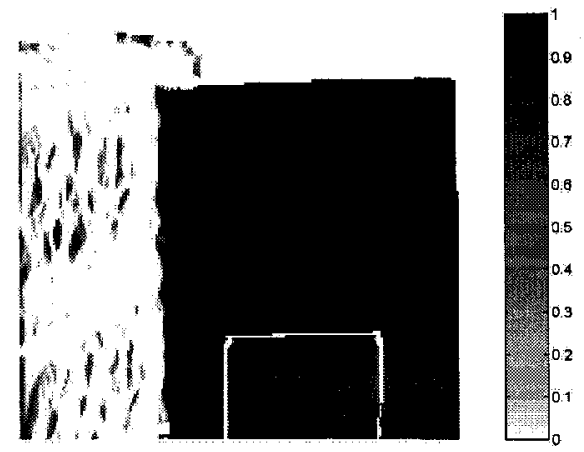

Figure E-9: Experiment \#5 orientation quality map

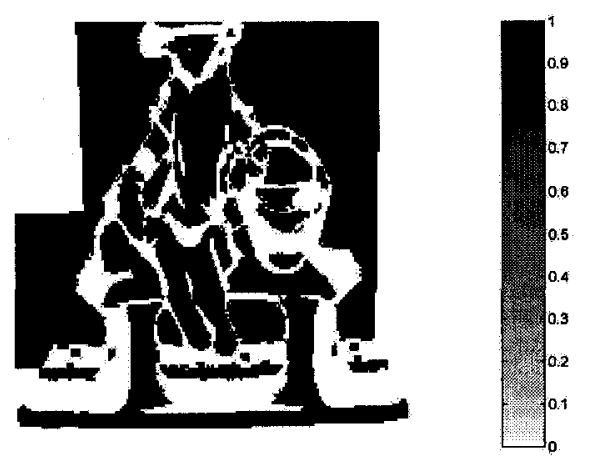

Figure E-8: Experiment \#4 resolvability quality map
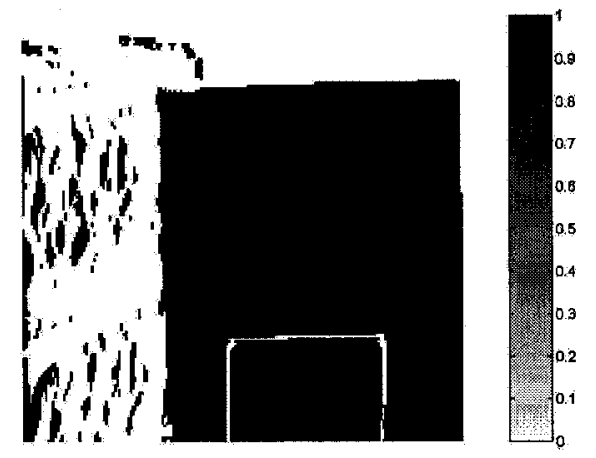

Figure E-10: Experiment \#5 resolvability quality map 


\section{Appendix F: Beam-Surface}

\section{Intersection}

The surface dimensions of the region illuminated by the laser beam can be obtained for mid-field measurements if the surface is assumed to be strictly planar within the beam footprint. Consider Figure 3.8(a) which illustrates the intersection of the laser beam with a surface angled $\varrho_{i}$ with respect to the laser path. We can rewrite (2.13) as

$$
\zeta_{0}^{2} w(\zeta)^{2}=\zeta_{0}^{2} w_{0}^{2}+\zeta^{2} w_{0}^{2}
$$

where we have used $w_{0}=w(0)$ to simplify the equation.

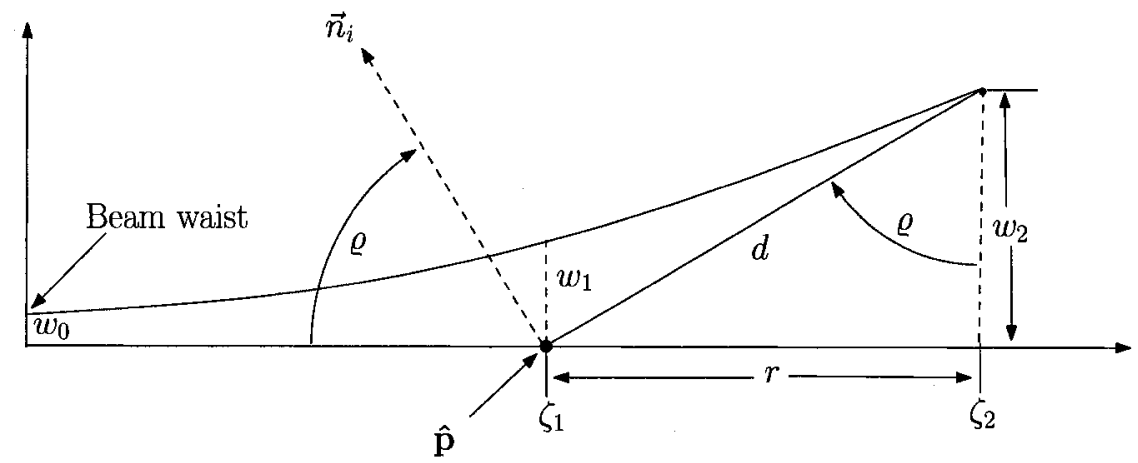

Figure F-1: Beam-surface intersection 
Figure F-1 illustrates the cross-section of the plane intersecting the laser beam. The plane intersects the centre of the beam at a distance $\zeta_{1}$ from the beam waist, and intersects the $1 / e^{2}$ boundary at $\zeta_{2}$ from the beam waist. We use $w_{1}=w\left(\zeta_{1}\right)$ and $w_{2}=w\left(\zeta_{2}\right)$ to represent the widths of the beam at a distance $\zeta_{1}$ and $\zeta_{2}$ respectively. The term $\zeta_{1}$ also represents the distance between the beam waist and the spatial location of the measurement generated by the reflected beam footprint so can be easily obtained. Similarly, $w_{1}$ represents the spot radius corresponding to the measurement $\hat{\mathbf{p}}$ so is also easily obtained. Finally, $\varrho$ is estimated from the surface normal $\vec{n}_{i}$ of the neighbourhood regression plane through $\hat{\mathbf{p}}$. What remains is to find $d$, the surface distance between the peak and edge of the beam footprint.

The terms $\zeta_{2}$ and $w_{2}$ are unknown so need to be eliminated. From Figure F-1, we see that

$$
\zeta_{2}=\zeta_{1}+r
$$

where

$$
r=d \sin (\varrho)
$$

SO

$$
\zeta_{2}=\zeta_{1}+d \sin (\varrho)
$$

in which all the terms in the right-hand side are known. Similarly

$$
w_{2}=d \cos (\varrho)
$$

is expressed in terms of $d$ and $\varrho$ which are also known. Substituting (F-4) and (F-5) into (F-1) results in

$$
\zeta_{0}^{2} d^{2} \cos ^{2}(\varrho)=\zeta_{0}^{2} w_{0}^{2}+w_{0}^{2}\left(\zeta_{1}+d \sin (\varrho)\right)^{2} .
$$


Equation (F-6) can be expanded in terms of $d$ into an equation of the form

$$
d^{2} K_{1}+d K_{2}+K_{3}=0
$$

where

$$
\begin{aligned}
K_{1} & =\left[w_{0} \sin (\varrho)\right]^{2}-\left[\zeta_{0} \cos (\varrho)\right]^{2}, \\
K_{2} & =2 \zeta_{1} w_{0}^{2} \sin (\varrho),
\end{aligned}
$$

and

$$
K_{3}=\left[w_{0} \zeta_{0}\right]^{2}+\left[w_{0} \zeta_{1}\right]^{2}
$$

Equation (F-7) can now be solved using a quadratic equation, generating two possible values of $d$. These values are the lengths of the long and short axes, representing the cross-section of the intersection of the surface with the $1 / e^{2}$ boundary. Specifically,

$$
\left\{d_{\min }, d_{\max }\right\}=\left|\frac{-K_{2} \pm \sqrt{\left(K_{2}\right)^{2}-4 K_{1} K_{3}}}{2 K_{1}}\right| .
$$

Equation (F-1) is symmetrical about the beam waist so this approach holds throughout the mid-field regardless of whether the surface is closer to or farther from the laser with respect to the beam waist. As $\varrho \rightarrow 0, d_{\max } \rightarrow d_{\min }$ and $d_{l e n g t h}=d_{\max }+d_{\min } \rightarrow d_{w i d t h}=$ $2 w_{1}$ where $d_{\text {length }}$ is the length of the ovoid beam footprint and $d_{w i d t h}$ is the width of the ovoid beam footprint. 


\section{Appendix G: Scanner Positioning}

Table G-1: Experiment \#1 scanner placement

\begin{tabular}{|ccccc|}
\hline $\begin{array}{c}\text { Reposition } \\
\text { Step }\end{array}$ & $\begin{array}{c}\text { Initial } \\
W^{\text {spot }}\end{array}$ & $\begin{array}{c}\text { Predicted } \\
W^{\text {spot }}\end{array}$ & $\begin{array}{c}\text { Requested } \\
\text { Translation }(\mathrm{m})\end{array}$ & $\begin{array}{c}\text { Requested } \\
\text { Rotation }\end{array}$ \\
\hline \hline 1 & 8.332 & 0.136 & $\mathrm{X}=0.0 / \mathrm{Y}=0.0 / \mathrm{Z}=1.0$ & $\theta=2^{\circ} / \phi=12^{\circ}$ \\
2 & 0.414 & 0.305 & $\mathrm{X}=0.0 / \mathrm{Y}=0.0 / \mathrm{Z}=-0.2$ & $\theta=4^{\circ} / \phi=2^{\circ}$ \\
3 & 0.268 & 0.268 & $\mathrm{X}=0.0 / \mathrm{Y}=0.0 / \mathrm{Z}=0.0$ & $\theta=1^{\circ} / \phi=3^{\circ}$ \\
\hline
\end{tabular}

Table G-2: Experiment \#2 scanner placement

\begin{tabular}{|ccccc|}
\hline $\begin{array}{c}\text { Reposition } \\
\text { Step }\end{array}$ & $\begin{array}{c}\text { Initial } \\
W^{\text {spot }}\end{array}$ & $\begin{array}{c}\text { Predicted } \\
W^{\text {spot }}\end{array}$ & $\begin{array}{c}\text { Requested } \\
\text { Translation }(\mathrm{m})\end{array}$ & $\begin{array}{c}\text { Requested } \\
\text { Rotation }\end{array}$ \\
\hline \hline 1 & 0.277 & 0.277 & $\mathrm{X}=0.0 / \mathrm{Y}=0.0 / \mathrm{Z}=0.0$ & $\theta=-2^{\circ} / \phi=2^{\circ}$ \\
\hline
\end{tabular}

Table G-3: Experiment \#3 scanner placement

\begin{tabular}{|ccccc|}
\hline $\begin{array}{c}\text { Reposition } \\
\text { Step }\end{array}$ & $\begin{array}{c}\text { Initial } \\
W^{\text {spot }}\end{array}$ & $\begin{array}{c}\text { Predicted } \\
W^{\text {spot }}\end{array}$ & $\begin{array}{c}\text { Requested } \\
\text { Translation }(\mathrm{m})\end{array}$ & $\begin{array}{c}\text { Requested } \\
\text { Rotation }\end{array}$ \\
\hline \hline 1 & 0.591 & 0.318 & $\mathrm{X}=0.0 / \mathrm{Y}=0.0 / \mathrm{Z}=0.4$ & $\theta=3^{\circ} / \phi=2^{\circ}$ \\
2 & 0.586 & 0.518 & $\mathrm{X}=-0.1 / \mathrm{Y}=0.0 / \mathrm{Z}=-0.1$ & $\theta=-1^{\circ} / \phi=2^{\circ}$ \\
3 & 0.436 & 0.436 & $\mathrm{X}=0.0 / \mathrm{Y}=0.0 / \mathrm{Z}=0.0$ & $\theta=0^{\circ} / \phi=2^{\circ}$ \\
\hline
\end{tabular}


Table G-4: Experiment \#4 scanner placement

\begin{tabular}{|ccccc|}
\hline $\begin{array}{c}\text { Reposition } \\
\text { Step }\end{array}$ & $\begin{array}{c}\text { Initial } \\
W^{\text {spot }}\end{array}$ & $\begin{array}{c}\text { Predicted } \\
W^{\text {spot }}\end{array}$ & $\begin{array}{c}\text { Requested } \\
\text { Translation }(\mathrm{m})\end{array}$ & $\begin{array}{c}\text { Requested } \\
\text { Rotation }\end{array}$ \\
\hline \hline 1 & 7.773 & 0.473 & $\mathrm{X}=0.0 / \mathrm{Y}=0.0 / \mathrm{Z}=1.0$ & $\theta=4^{\circ} / \phi=12^{\circ}$ \\
2 & 0.791 & 0.473 & $\mathrm{X}=0.0 / \mathrm{Y}=0.0 / \mathrm{Z}=-0.3$ & $\theta=1^{\circ} / \phi=1^{\circ}$ \\
3 & 0.441 & 0.441 & $\mathrm{X}=0.0 / \mathrm{Y}=0.0 / \mathrm{Z}=0.0$ & $\theta=1^{\circ} / \phi=2^{\circ}$ \\
\hline
\end{tabular}

Table G-5: Experiment \#5 scanner placement

\begin{tabular}{|ccccc|}
\hline $\begin{array}{c}\text { Reposition } \\
\text { Step }\end{array}$ & $\begin{array}{c}\text { Initial } \\
W^{\text {spot }}\end{array}$ & $\begin{array}{c}\text { Predicted } \\
W^{\text {spot }}\end{array}$ & $\begin{array}{c}\text { Requested } \\
\text { Translation }(\mathrm{m})\end{array}$ & $\begin{array}{c}\text { Requested } \\
\text { Rotation }\end{array}$ \\
\hline \hline 1 & 7.305 & 0.586 & $\mathrm{X}=0.0 / \mathrm{Y}=0.0 / \mathrm{Z}=0.9$ & $\theta=4^{\circ} / \phi=11^{\circ}$ \\
2 & 0.327 & 0.277 & $\mathrm{X}=0.0 / \mathrm{Y}=0.0 / \mathrm{Z}=-0.1$ & $\theta=-1^{\circ} / \phi=2^{\circ}$ \\
3 & 0.332 & 0.332 & $\mathrm{X}=0.0 / \mathrm{Y}=0.0 / \mathrm{Z}=0.0$ & $\theta=-1^{\circ} / \phi=3^{\circ}$ \\
\hline
\end{tabular}




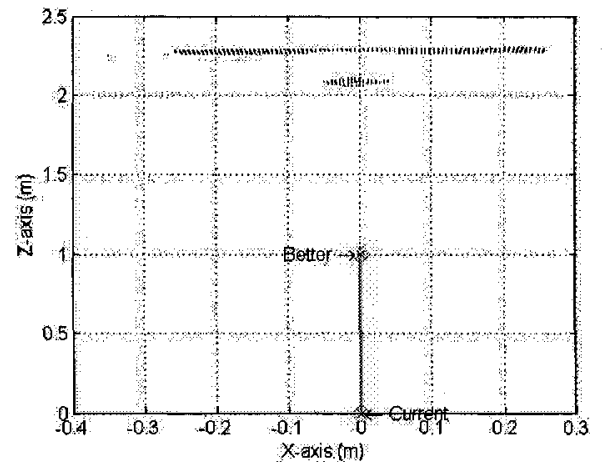

Figure G-1: Experiment \#1 repositioning 1

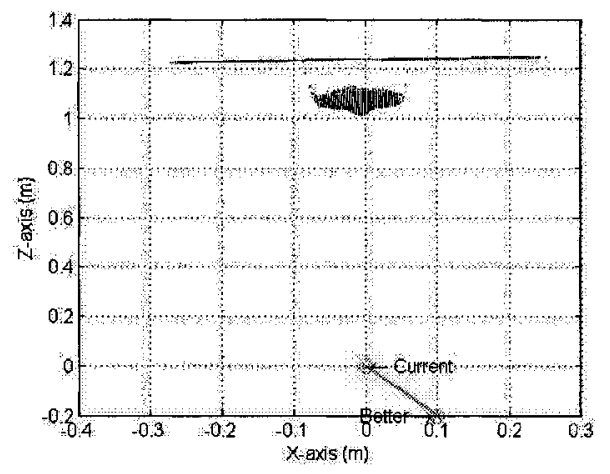

Figure G-2: Experiment \#1 repositioning 2

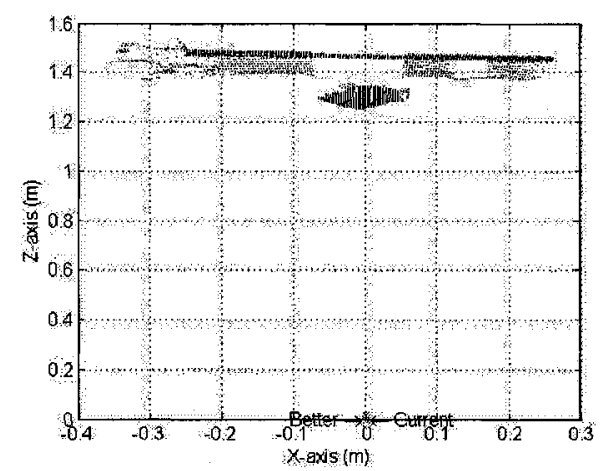

Figure G-3: Experiment \#1 repositioning 3 


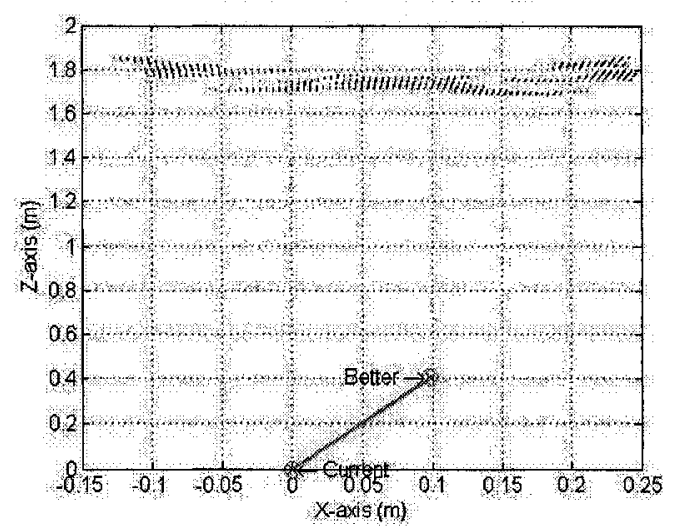

Figure G-4: Experiment \#3 repositioning 1

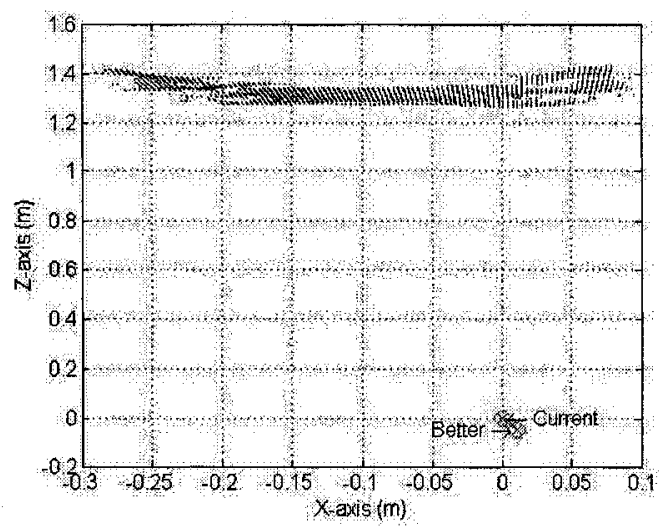

Figure G-5: Experiment \#3 repositioning 2

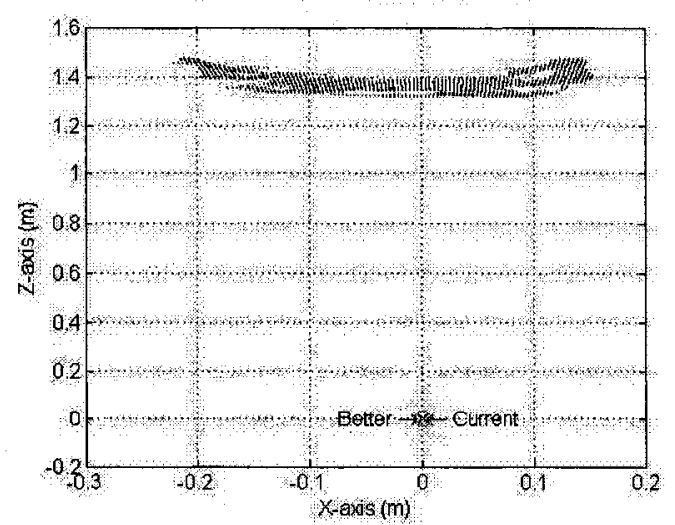

Figure G-6: Experiment \#3 repositioning 3 


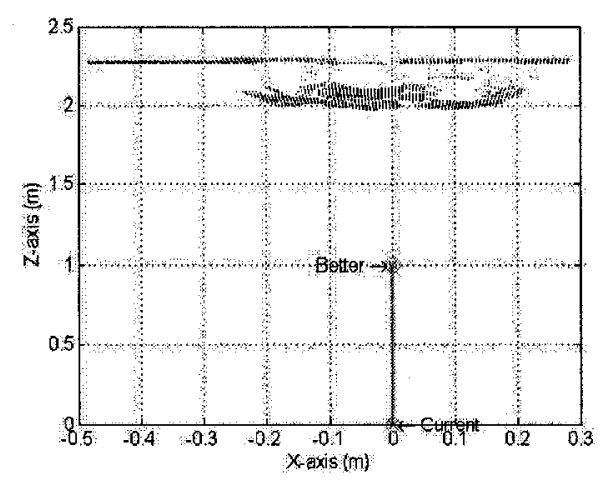

Figure G-7: Experiment \#4 repositioning 1

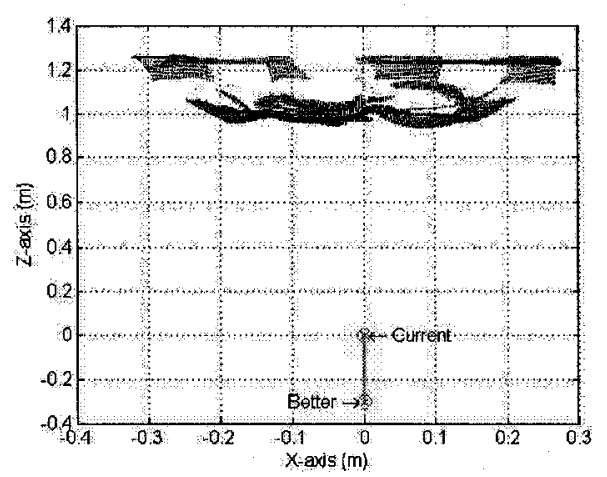

Figure G-8: Experiment \#4 repositioning 2

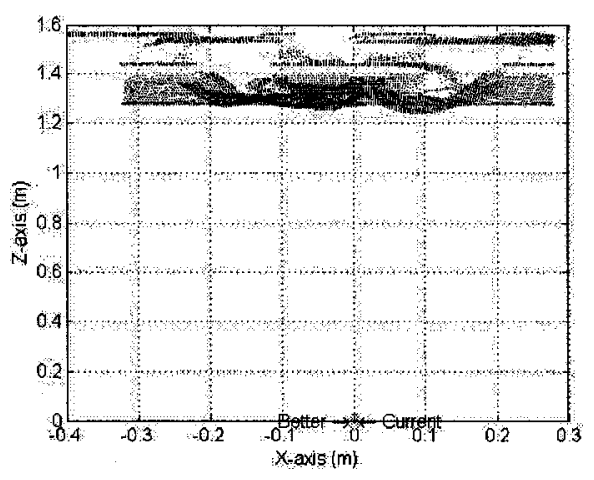

Figure G-9: Experiment \#4 repositioning 3 


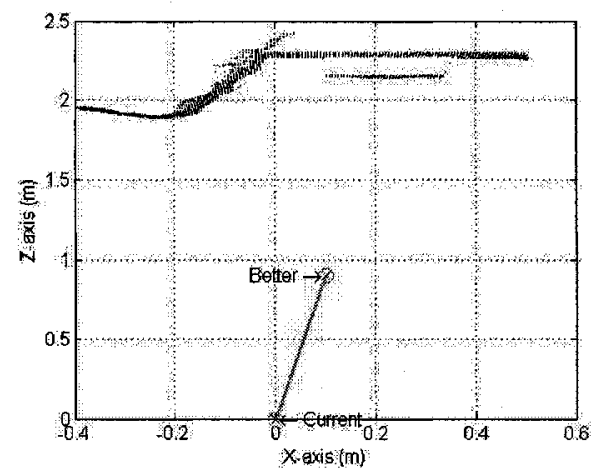

Figure G-10: Experiment \#5 repositioning 1

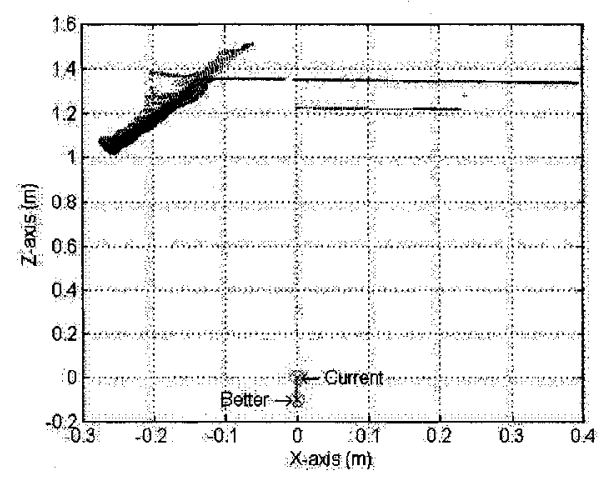

Figure G-11: Experiment \#5 repositioning 2

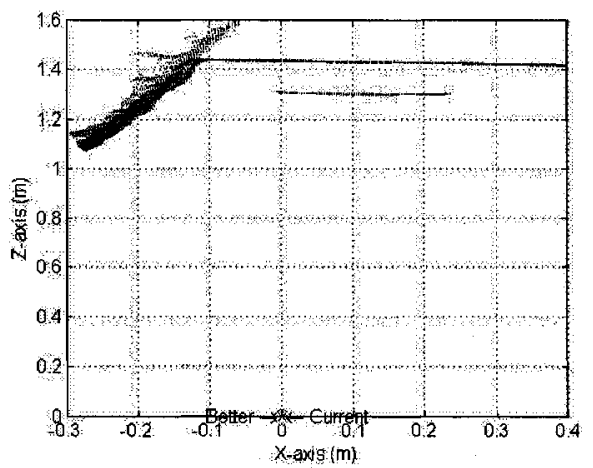

Figure G-12: Experiment \#5 repositioning 3 


\section{Appendix H: Reflectivity and Planarity Quality Maps}



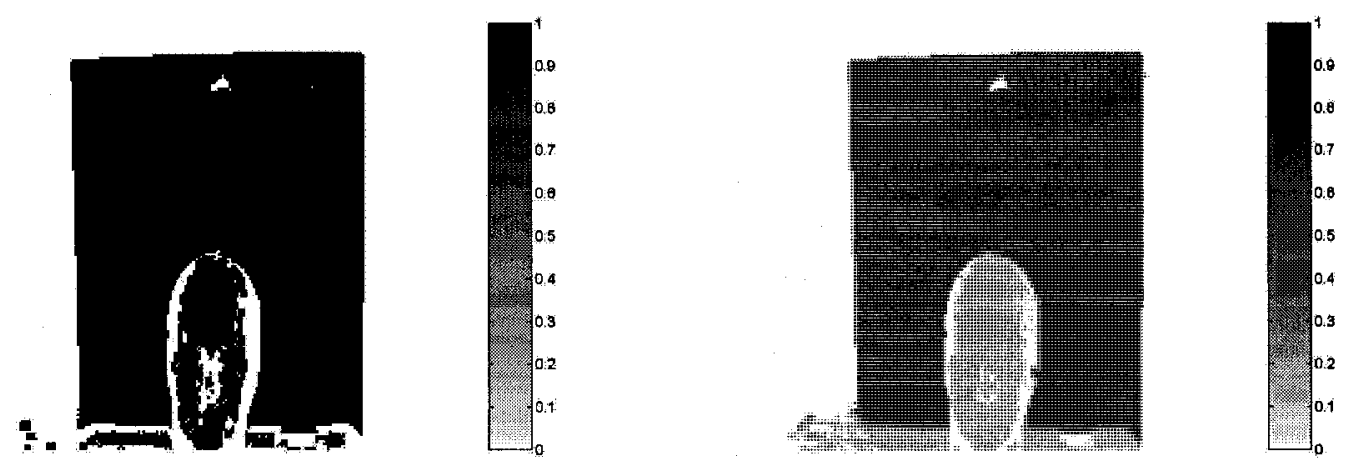

Figure H-1: Experiment \#1 prereflectivity planarity quality map Figure H-2: Experiment \#1 reflectivity quality map
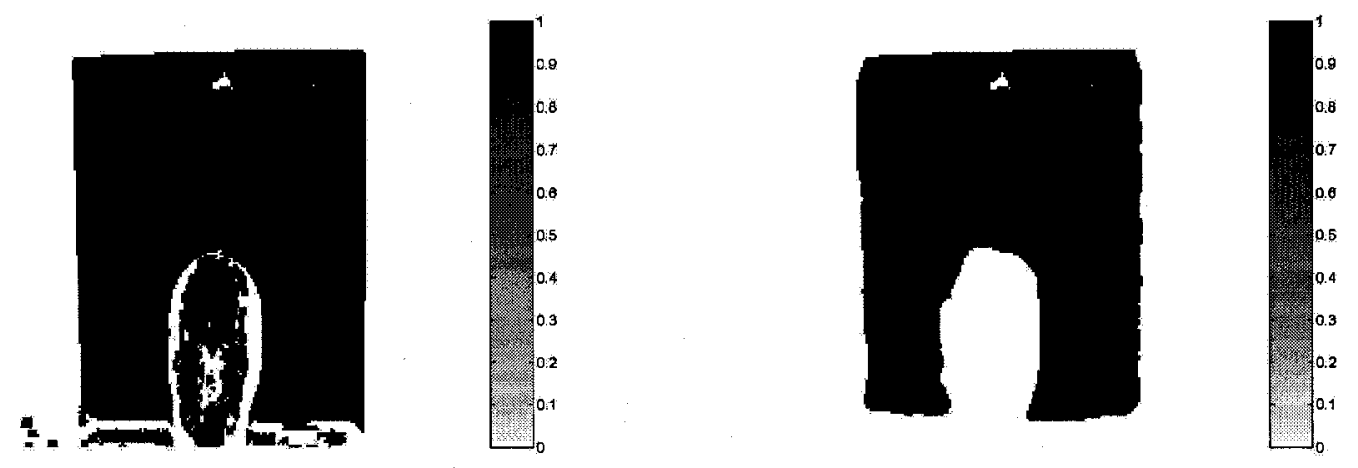

Figure H-3: Experiment \#1 postreflectivity planarity quality map

Figure H-4: Experiment \#1 post-outlier planarity quality map 


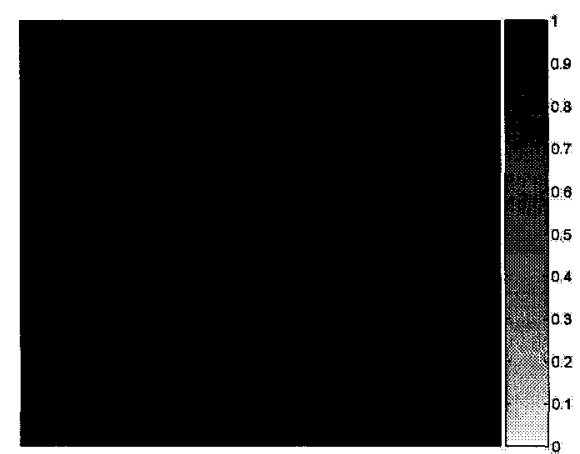

Figure H-5: Experiment \#2 prereflectivity planarity quality map

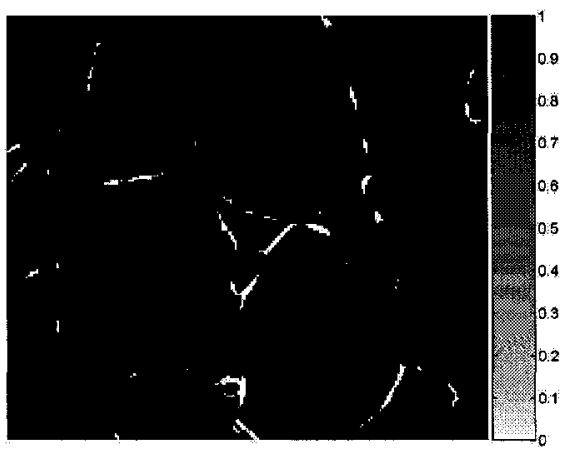

Figure H-7: Experiment \#2 postreflectivity planarity quality map

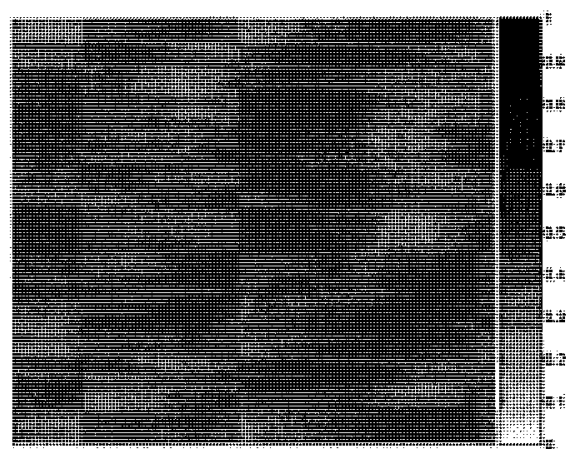

Figure H-6: Experiment \#2 reflectivity quality map

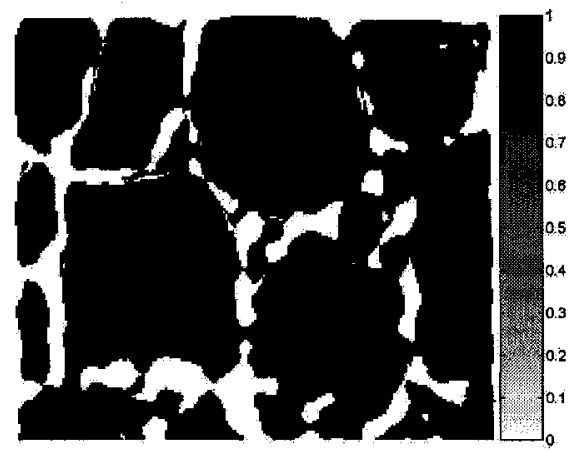

Figure H-8: Experiment \#2 post-outlier planarity quality map 

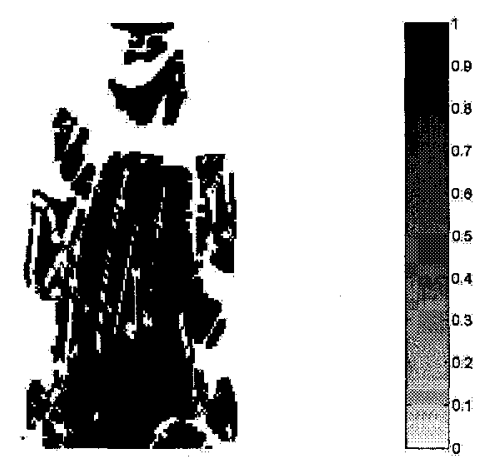

Figure H-9: Experiment \#3 prereflectivity planarity quality map
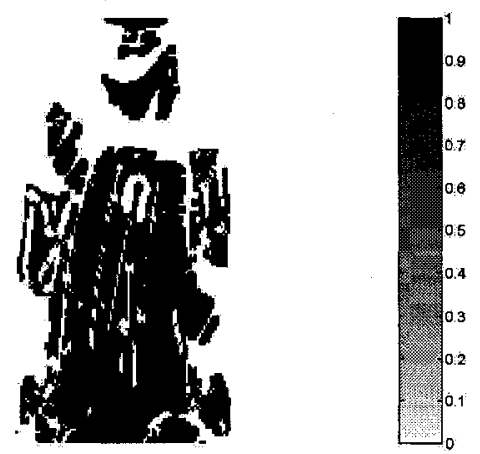

Figure H-11: Experiment \#3 postreflectivity planarity quality map
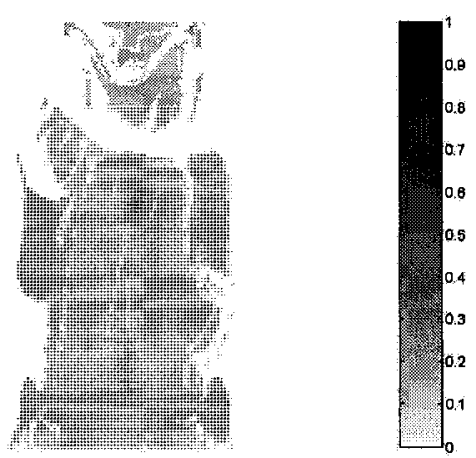

Figure H-10: Experiment \#3 reflectivity quality map

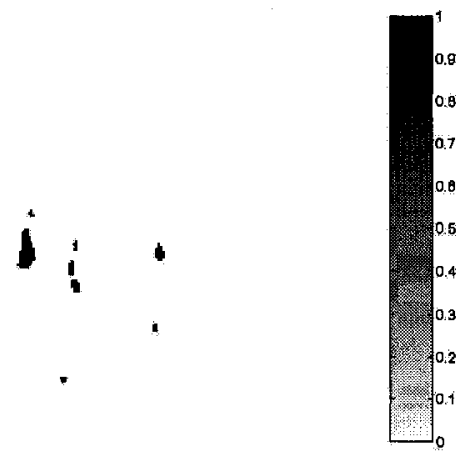

Figure H-12: Experiment \#3 post-outlier planarity quality map 

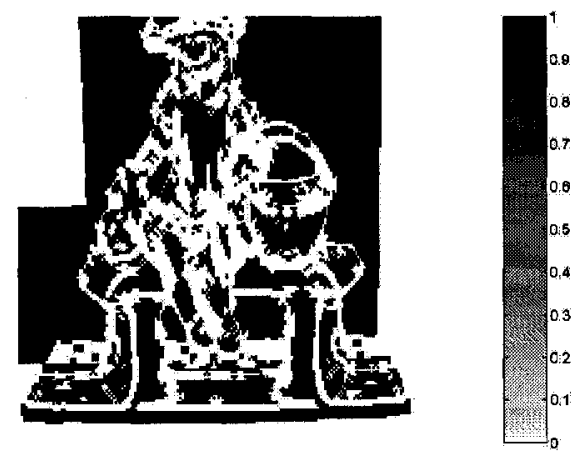

Figure H-13: Experiment \#4 prereflectivity planarity quality map
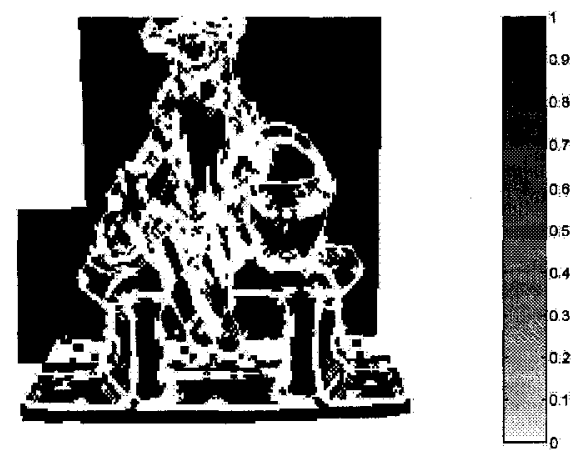

Figure H-15: Experiment \#4 postreflectivity planarity quality map

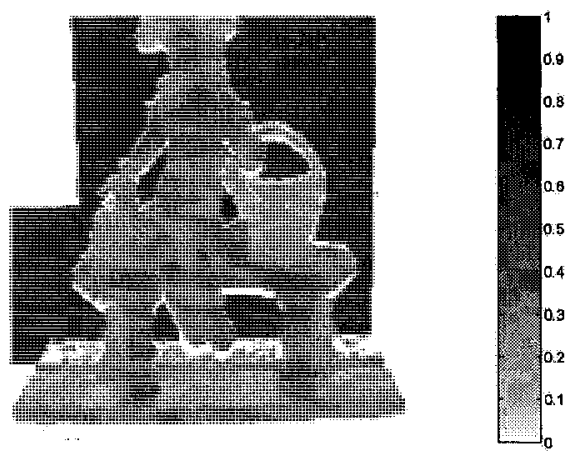

Figure H-14: Experiment \#4 reflectivity quality map
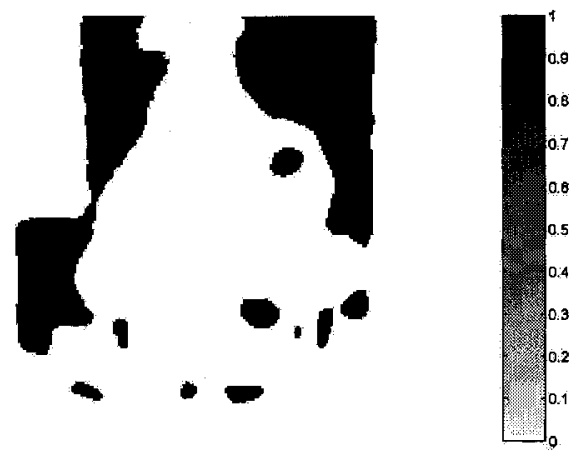

Figure H-16: Experiment \#4 post-outlier planarity quality map 

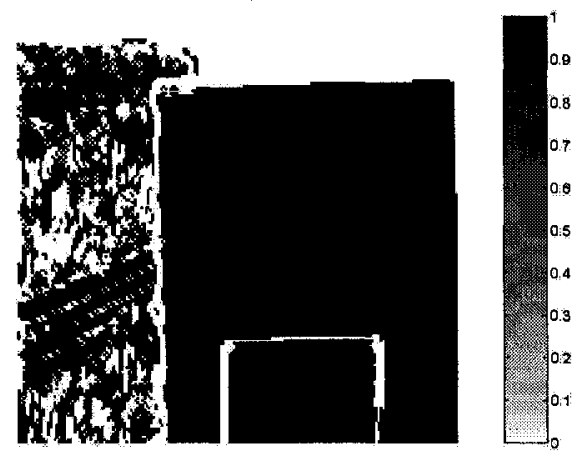

Figure H-17: Experiment \#5 prereflectivity planarity quality map
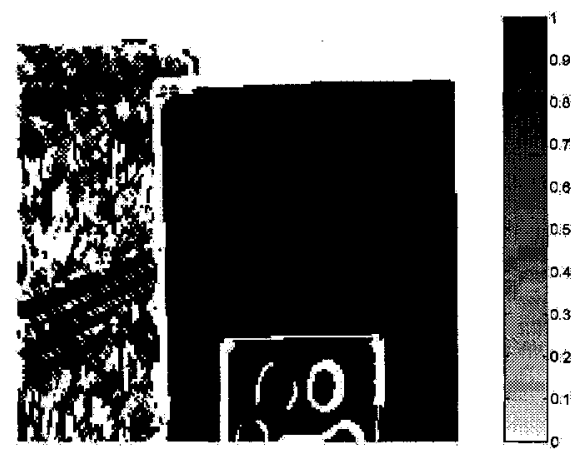

Figure H-19: Experiment \#5 postreflectivity planarity quality map

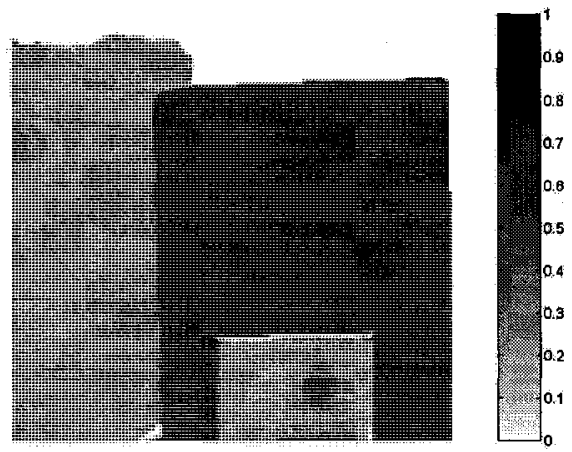

Figure H-18: Experiment \#5 reflectivity quality map

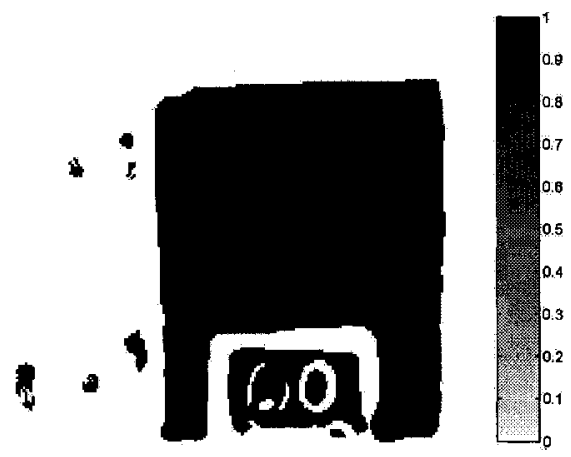

Figure H-20: Experiment \#5 post-outlier planarity quality map 


\section{Appendix I: Regions Maps and}

\section{Subscans}

Table I-1: Scan time, processing time and number of measurements for full and reduced scans

\begin{tabular}{|l|ccccc|}
\hline \multicolumn{1}{|c|}{ Experiment } & 1 & 2 & 3 & 4 & 5 \\
\hline Full Scan & \multicolumn{5}{|c|}{} \\
\hline Scan + Processing & $456.68 \mathrm{~min}$ & $456.68 \mathrm{~min}$ & $456.68 \mathrm{~min}$ & $456.68 \mathrm{~min}$ & $456.68 \mathrm{~min}$ \\
\# Measurements & $25,396,875$ & $25,396,875$ & $25,396,875$ & $25,396,875$ & $25,396,875$ \\
\hline Reduced Scan & & & & \\
\hline Scan + Processing & $189.4 \mathrm{~min}$ & $372.03 \mathrm{~min}$ & $141.38 \mathrm{~min}$ & $284.07 \mathrm{~min}$ & $284.07 \mathrm{~min}$ \\
\# Measurements & $11,513,250$ & $20,693,750$ & $9,519,125$ & $15,802,500$ & $15,802,500$ \\
\hline
\end{tabular}


Table I-2: Target-based Subscan efficiency versus full and reduced scan times and number of measurements (see Table I-1)

\begin{tabular}{|c|ccccc|}
\hline Experiment & 1 & 2 & 3 & 4 & 5 \\
\hline \# Anchor Scans & 3 & 1 & 3 & 3 & 3 \\
\# Subscans & 19 & 27 & 16 & 32 & 27 \\
\# Measurements & $1,441,792$ & $1,835,008$ & $1,245,184$ & $2,293,760$ & $1,966,080$ \\
\hline Scanning Time & $3.87 \mathrm{~min}$ & $4.93 \mathrm{~min}$ & $2.99 \mathrm{~min}$ & $5.81 \mathrm{~min}$ & $5.28 \mathrm{~min}$ \\
Processing Time & $26.56 \mathrm{~min}$ & $41.68 \mathrm{~min}$ & $25.69 \mathrm{~min}$ & $42.43 \mathrm{~min}$ & $38.84 \mathrm{~min}$ \\
\hline Total Stage 1 & $30.43 \mathrm{~min}$ & $46.61 \mathrm{~min}$ & $28.68 \mathrm{~min}$ & $48.24 \mathrm{~min}$ & $44.12 \mathrm{~min}$ \\
\hline Versus Full Scan & & & & & \\
\hline Time Efficiency & $6.7 \%$ & $10.2 \%$ & $6.3 \%$ & $10.6 \%$ & $9.7 \%$ \\
Sampling Efficiency & $5.7 \%$ & $7.2 \%$ & $4.9 \%$ & $9.0 \%$ & $7.7 \%$ \\
\hline Versus Reduced Scan & & & & & \\
\hline Time Efficiency & $16.1 \%$ & $12.5 \%$ & $20.3 \%$ & $17.0 \%$ & $15.5 \%$ \\
Sampling Efficiency & $12.5 \%$ & $8.9 \%$ & $13.1 \%$ & $14.5 \%$ & $12.4 \%$ \\
\hline
\end{tabular}


Table I-3: Spot-based Subscan efficiency versus full and reduced scan times and number of measurements (see Table I-1)

\begin{tabular}{|c|ccccc|}
\hline Experiment & 1 & 2 & 3 & 4 & 5 \\
\hline \# Anchor Scans & 3 & 1 & 3 & 3 & 3 \\
\# Subscans & 113 & 211 & 152 & 230 & 190 \\
\# Measurements & $7,602,176$ & $13,893,632$ & $10,158,080$ & $15,269,888$ & $12,648,448$ \\
\hline Scanning Time & $19.90 \mathrm{~min}$ & $37.16 \mathrm{~min}$ & $26.77 \mathrm{~min}$ & $40.50 \mathrm{~min}$ & $33.46 \mathrm{~min}$ \\
Processing Time & $118.99 \mathrm{~min}$ & $253.58 \mathrm{~min}$ & $150.75 \mathrm{~min}$ & $240.38 \mathrm{~min}$ & $186.29 \mathrm{~min}$ \\
\hline Total Stage 1 & $139.41 \mathrm{~min}$ & $290.89 \mathrm{~min}$ & $177.68 \mathrm{~min}$ & $281.04 \mathrm{~min}$ & $220.26 \mathrm{~min}$ \\
\hline Versus Full Scan & & & & & \\
\hline Time Efficiency & $30.5 \%$ & $63.7 \%$ & $38.9 \%$ & $61.5 \%$ & $48.2 \%$ \\
Sampling Efficiency & $29.9 \%$ & $54.7 \%$ & $40.0 \%$ & $60.1 \%$ & $49.8 \%$ \\
\hline Versus Reduced Scan & & & & & \\
\hline Time Efficiency & $73.6 \%$ & $78.2 \%$ & $125.7 \%$ & $98.9 \%$ & $77.5 \%$ \\
Sampling Efficiency & $66.0 \%$ & $67.1 \%$ & $106.7 \%$ & $96.6 \%$ & $80.0 \%$ \\
\hline
\end{tabular}




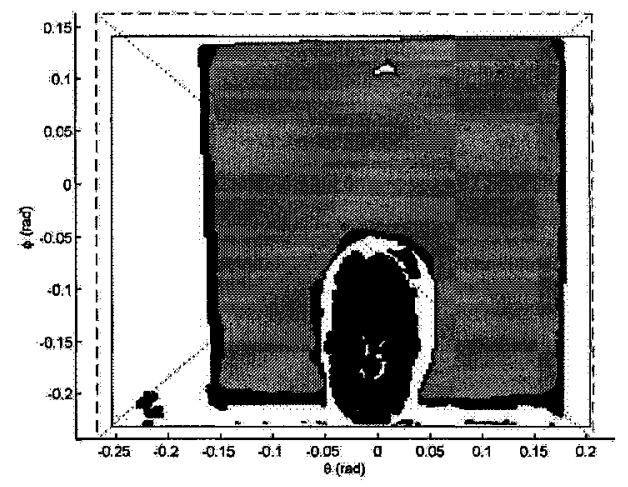

Figure I-1: Experiment \#1 region map

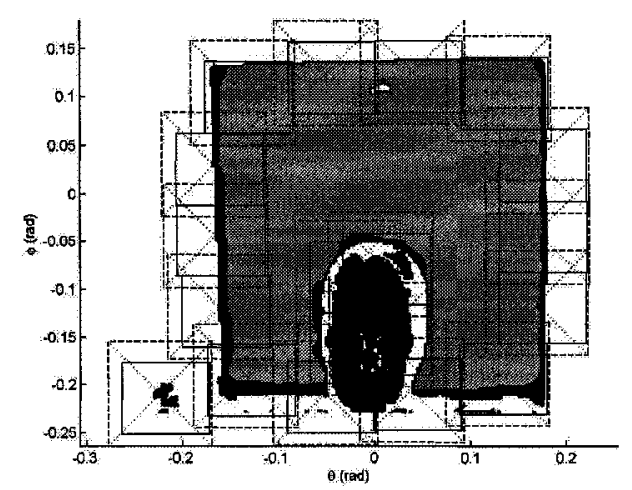

Figure I-2: Experiment \#1 target-based subscan map

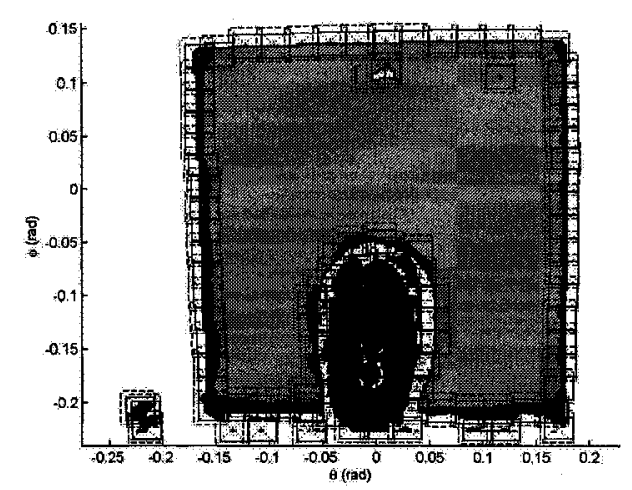

Figure I-3: Experiment \#1 spot-based subscan map 


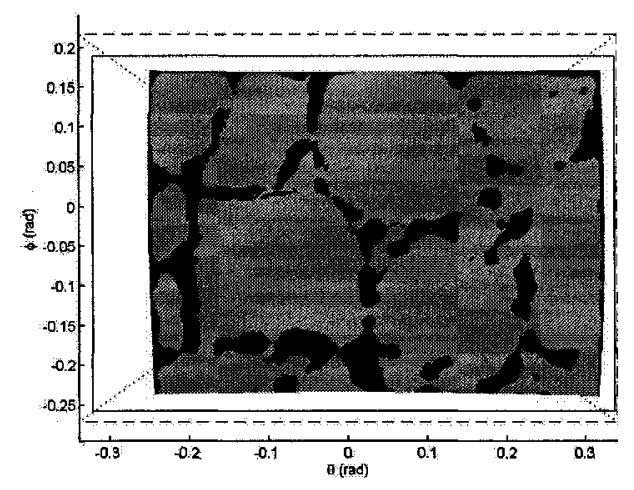

Figure I-4: Experiment \#2 region map

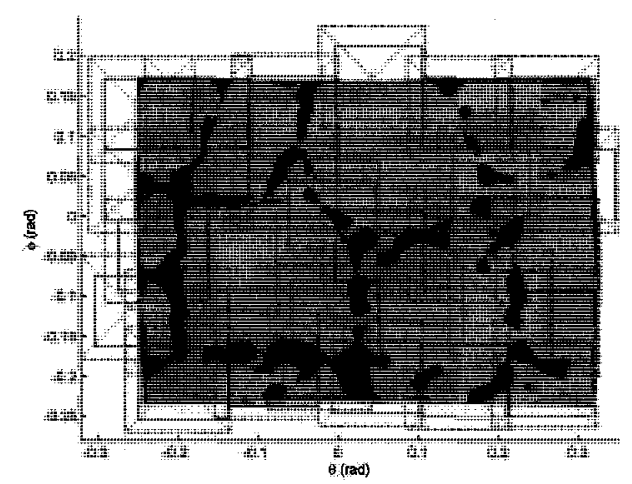

Figure I-5: Experiment \#2 target-based subscan map

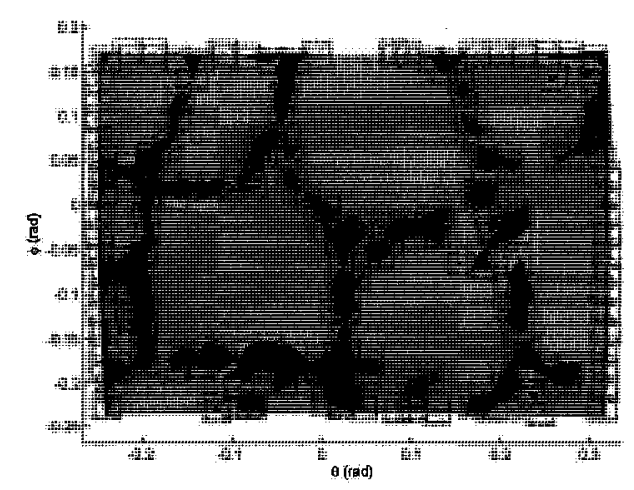

Figure I-6: Experiment \#2 spot-based subscan map 


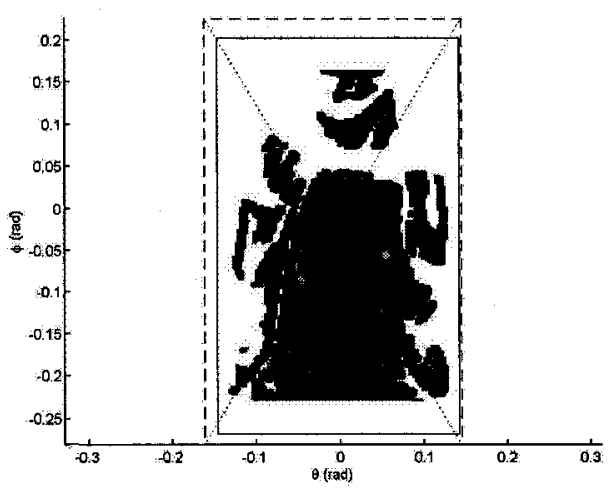

Figure I-7: Experiment \#3 region map

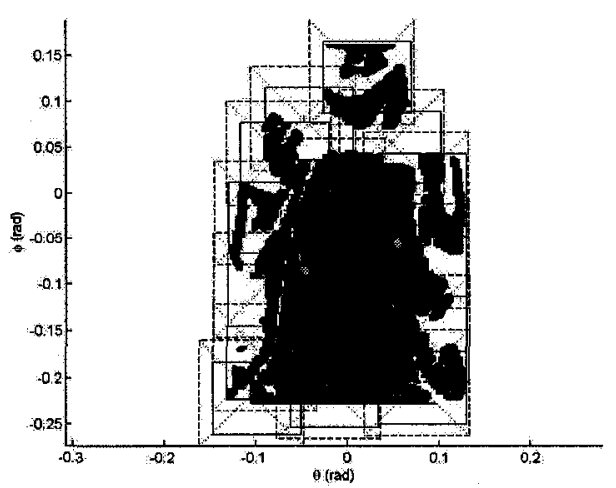

Figure I-8: Experiment \#3 target-based subscan map

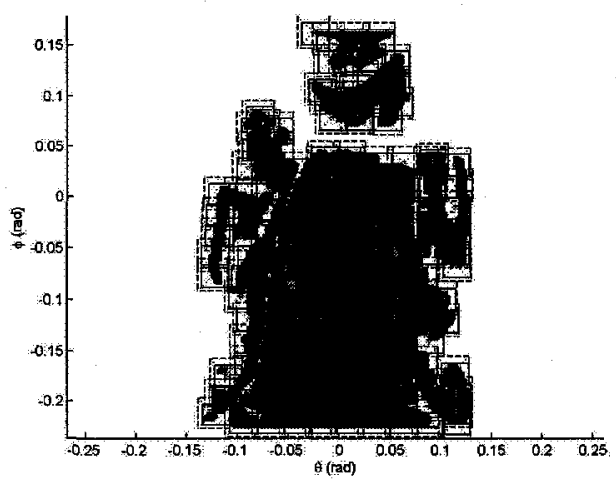

Figure I-9: Experiment \#3 spot-based subscan map 


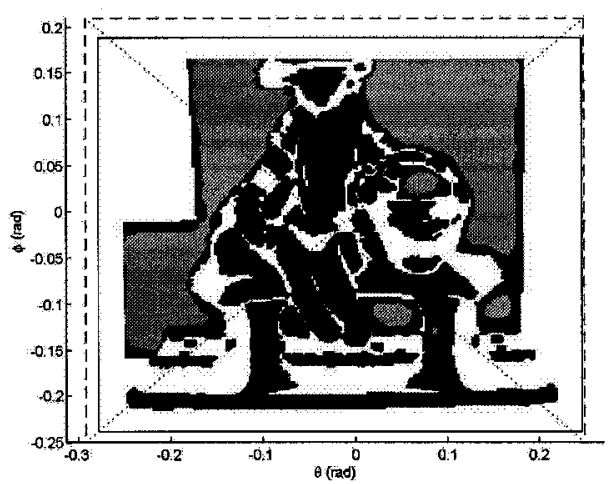

Figure I-10: Experiment \#4 region map

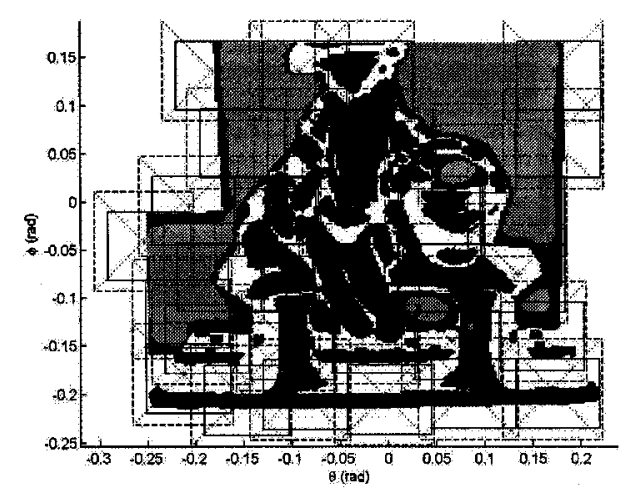

Figure I-11: Experiment \#4 target-based subscan map

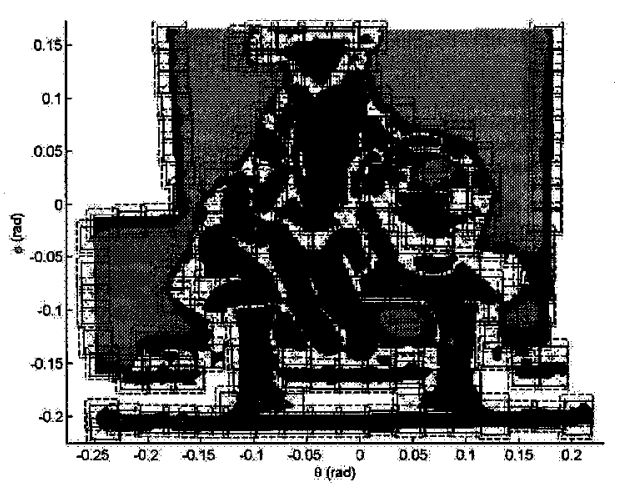

Figure I-12: Experiment \#4 spot-based subscan map 


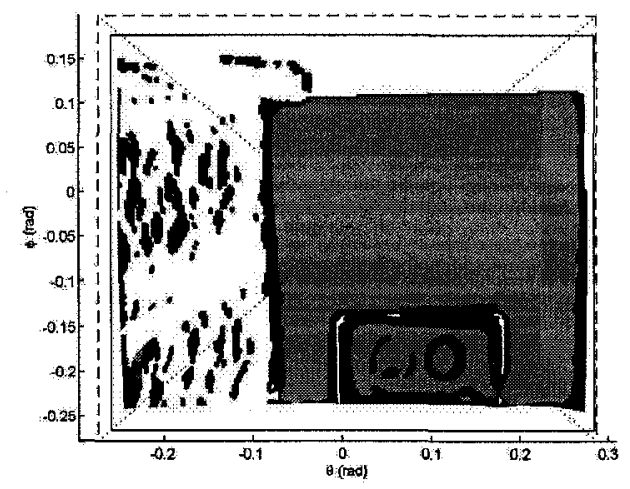

Figure I-13: Experiment \#5 region map

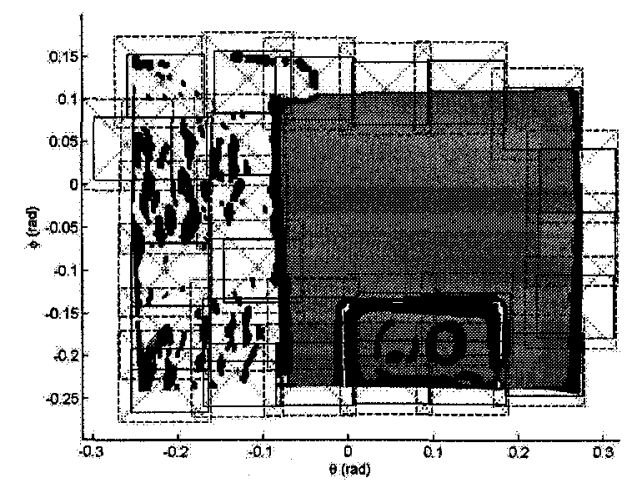

Figure I-14: Experiment \#5 target-based subscan map

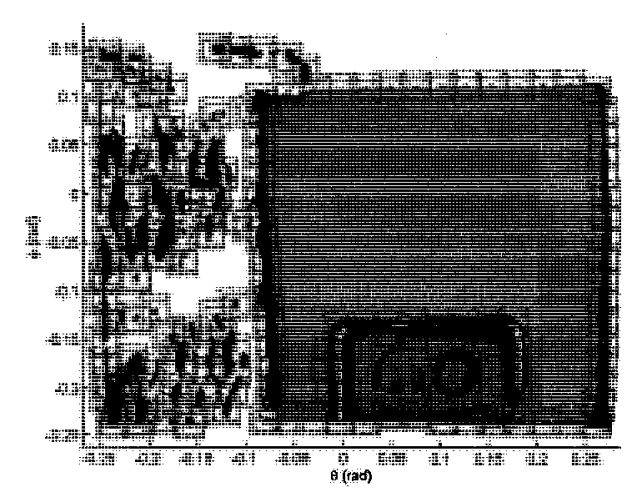

Figure I-15: Experiment \#5 spot-based subscan map 


\section{Appendix J: Aliasing, Integration, Completness and Total Quality Maps}



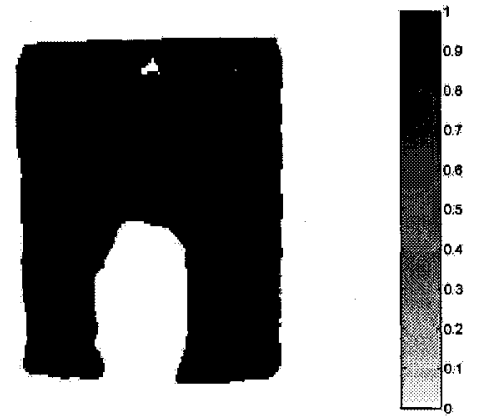

Figure J-1: Experiment \#1 aliasing quality map
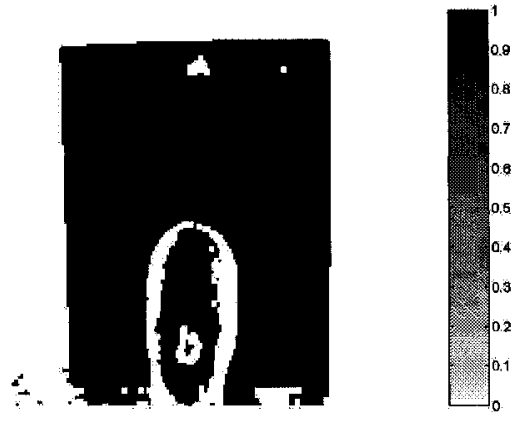

Figure J-3: Experiment \#1 enclosed quality map

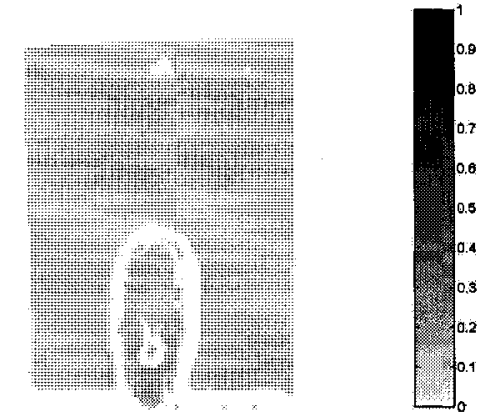

Figure J-2: Experiment \#1 integration quality map
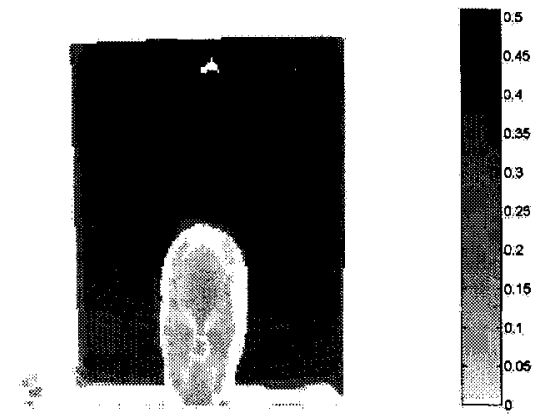

Figure J-4: Experiment \#1 within-scan total quality map

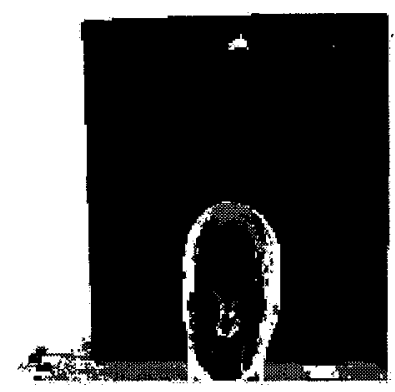

Figure J-5: Experiment \#1 acceptability map 


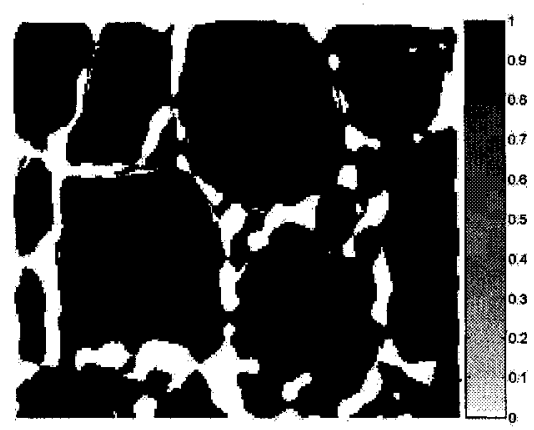

Figure J-6: Experiment \#2 aliasing quality map

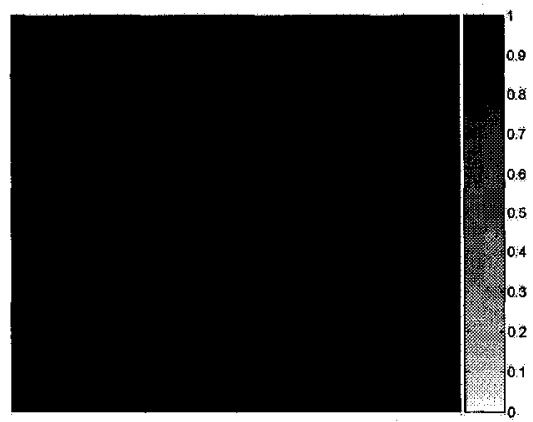

Figure J-8: Experiment \#2 enclosed quality map

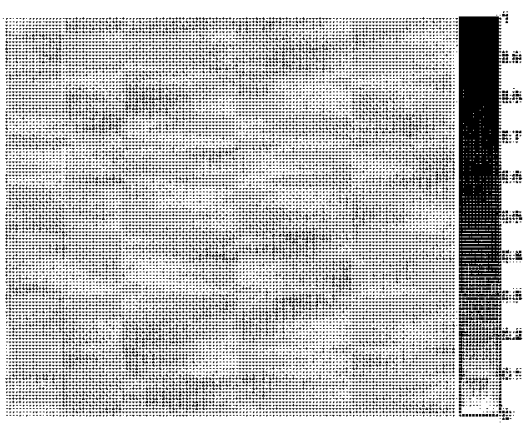

Figure J-7: Experiment \#2 integration quality map

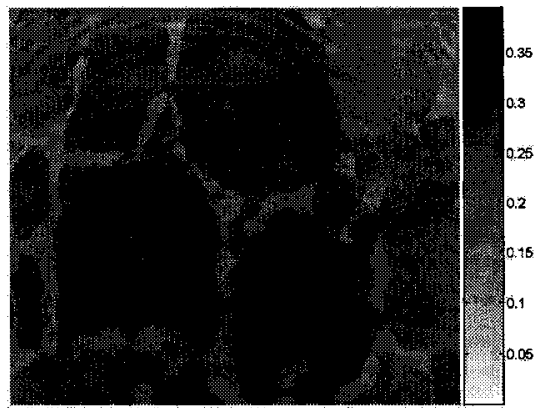

Figure J-9: Experiment \#2 within-scan Total quality map

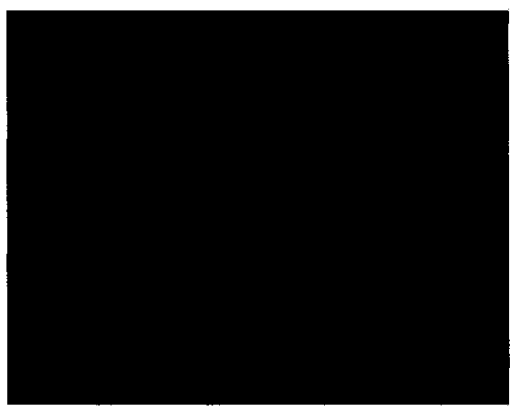

Figure J-10: Experiment \#2 acceptability map 


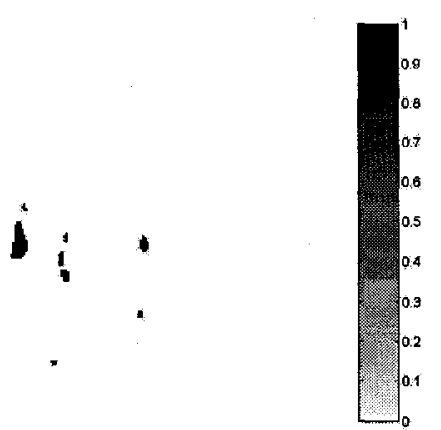

Figure J-11: Experiment \#3 aliasing quality map
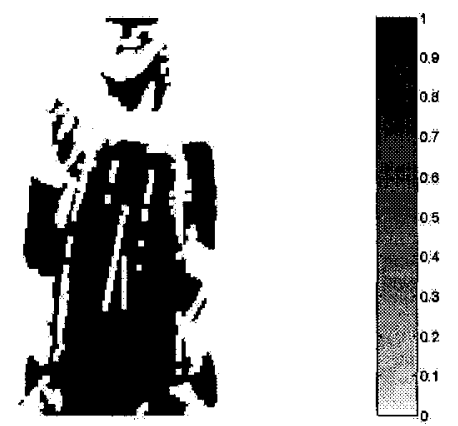
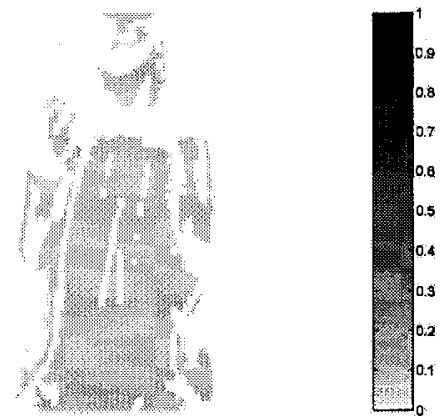

Figure J-12: Experiment \#3 integration quality map
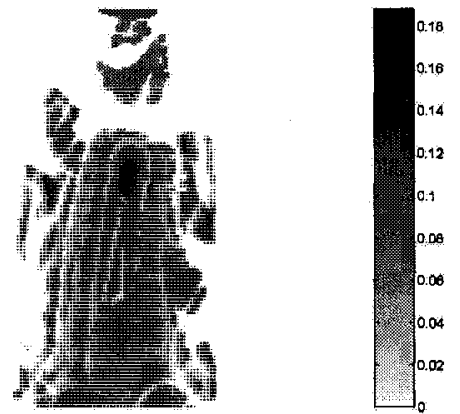

Figure J-13: Experiment \#3 enclosed Figure J-14: Experiment \#3 within-scan quality map total quality map

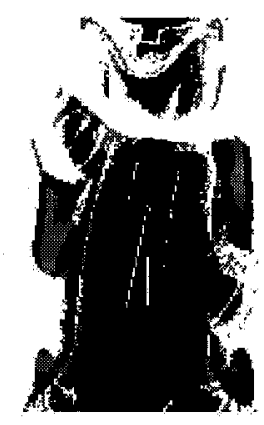

Figure J-15: Experiment \#3 acceptability map 

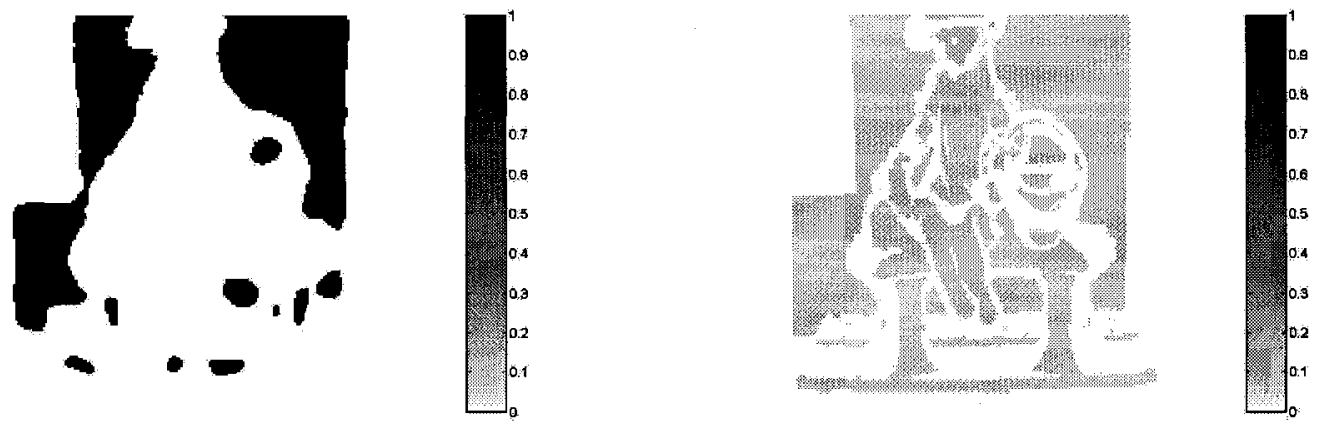

Figure J-16: Experiment \#4 aliasing quality map
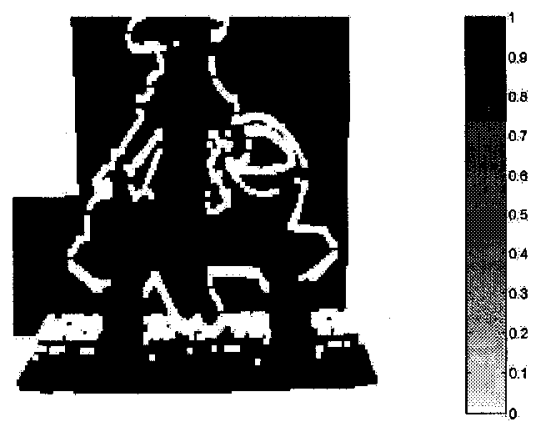

Figure J-17: Experiment \#4 integration quality map
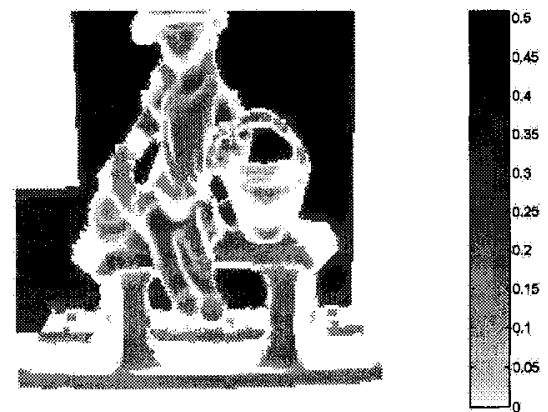

Figure J-18: Experiment \#4 enclosed Figure J-19: Experiment \#4 within-scan quality map total quality map

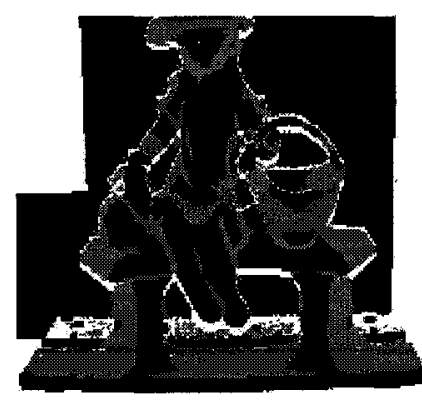

Figure J-20: Experiment \#4 acceptability map 


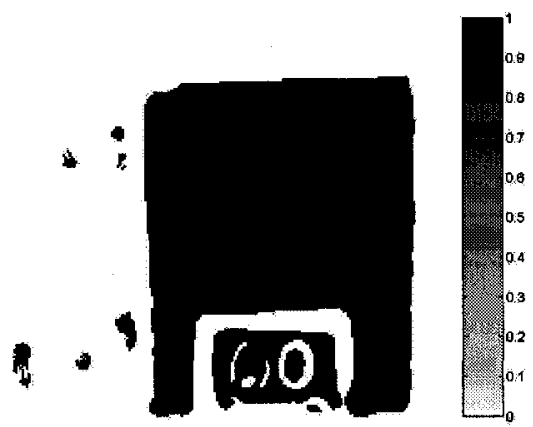

Figure J-21: Experiment \#5 aliasing quality map
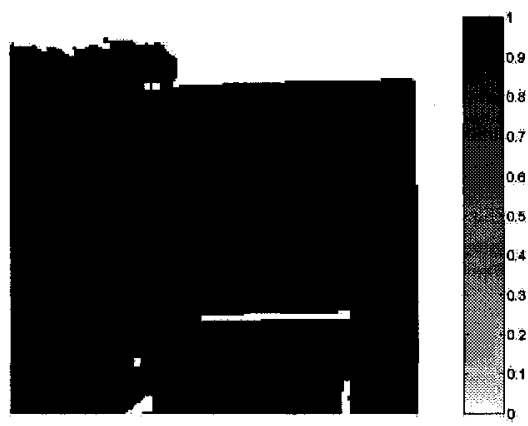

Figure J-23: Experiment \#5 enclosed quality map

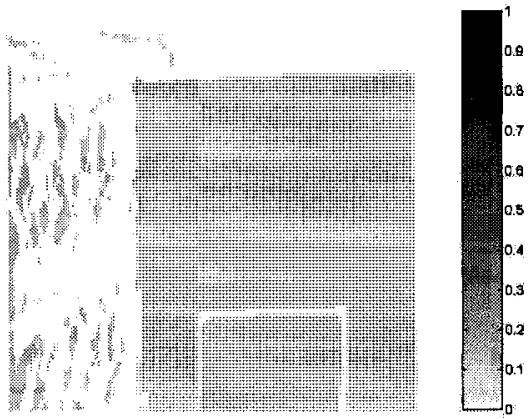

Figure J-22: Experiment \#5 integration quality map

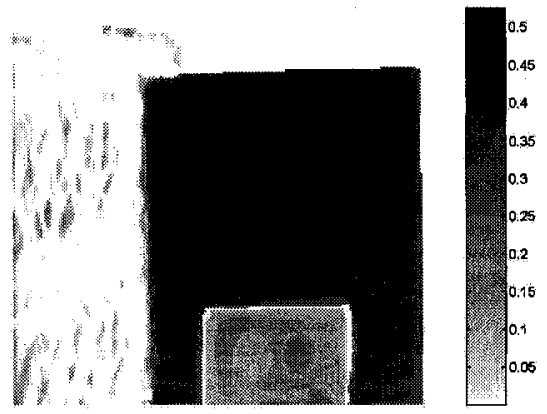

Figure J-24: Experiment \#5 within-scan total quality map

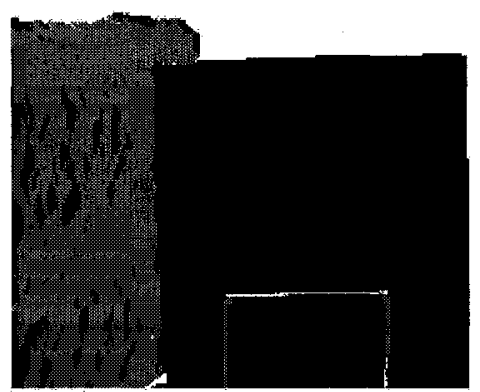

Figure J-25: Experiment \#5 acceptability map 


\section{Appendix K: Subscan Quality}




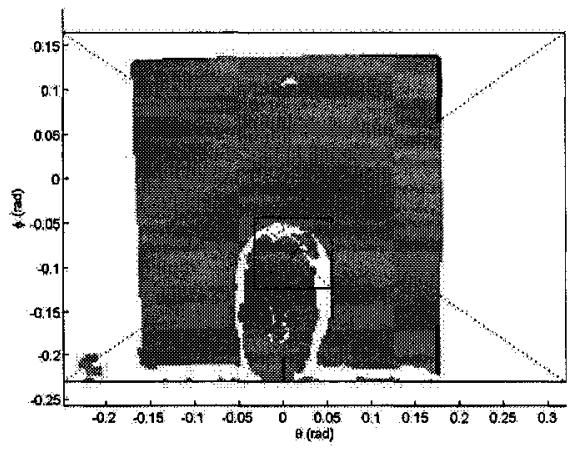

Figure K-1: Experiment \#1 subscan location in anchor

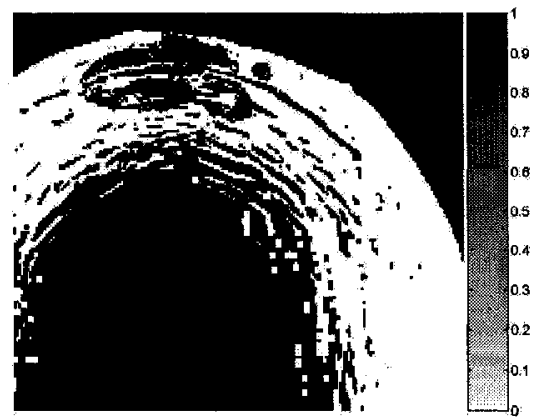

Figure K-3: Experiment \#1 subscan aliasing quality map

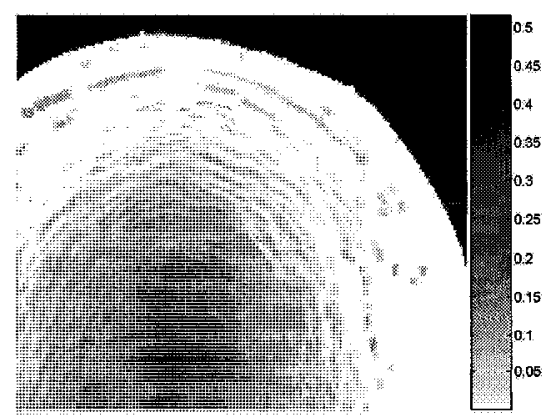

Figure K-5: Experiment \#1 subscan total quality map

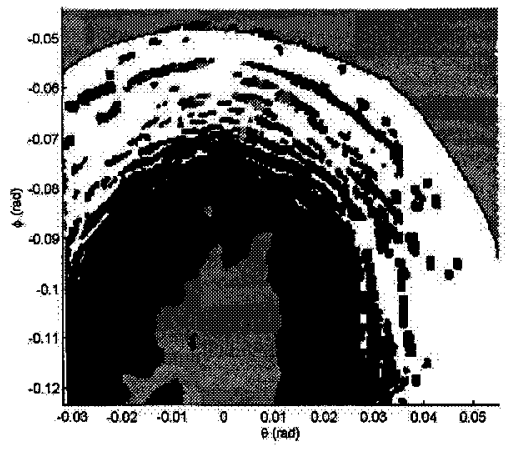

Figure K-2: Experiment \#1 subscan map

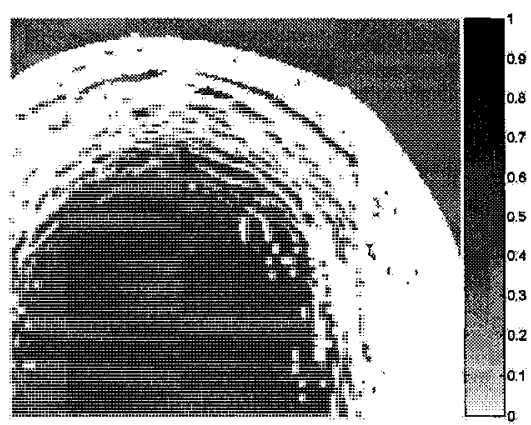

Figure K-4: Experiment \#1 subscan integration quality map

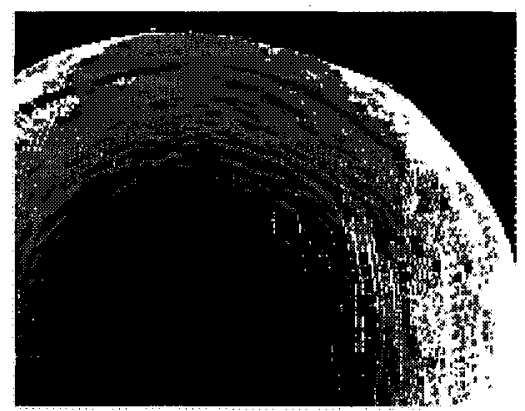

Figure K-6: Experiment \#1 subscan acceptability map 


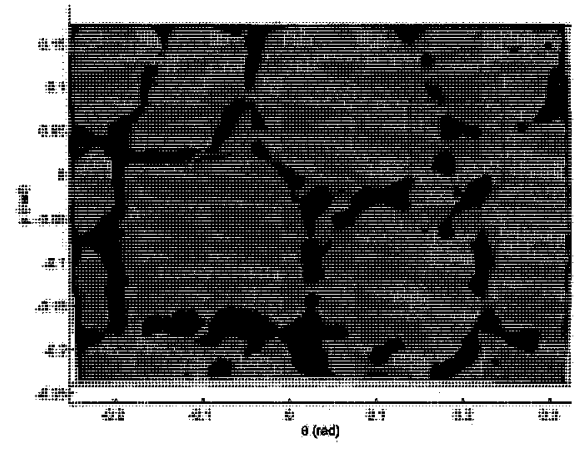

Figure K-7: Experiment \#2 subscan location in anchor

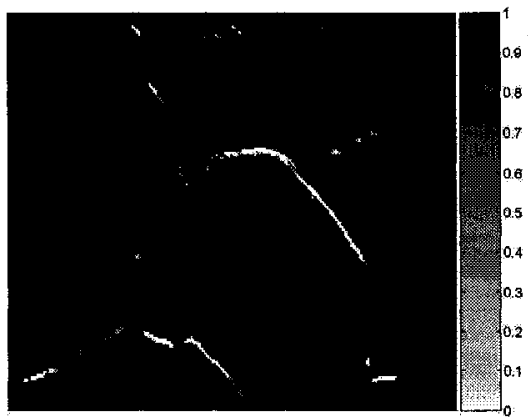

Figure K-9: Experiment \#2 subscan aliasing quality map

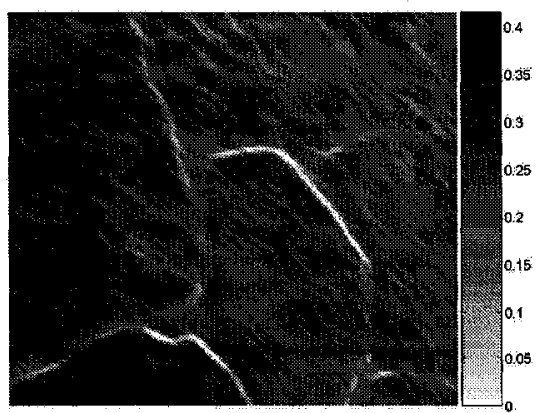

Figure K-11: Experiment \#2 subscan total quality map

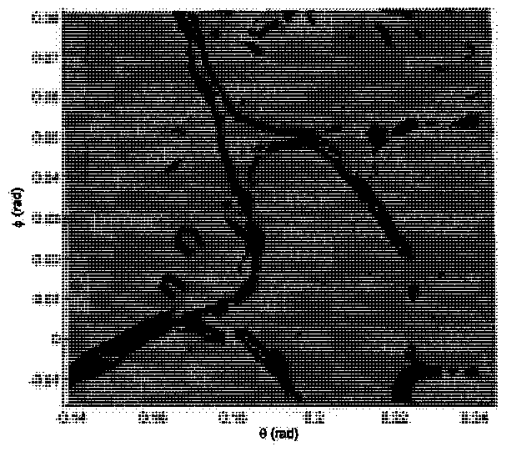

Figure K-8: Experiment \#2 subscan map

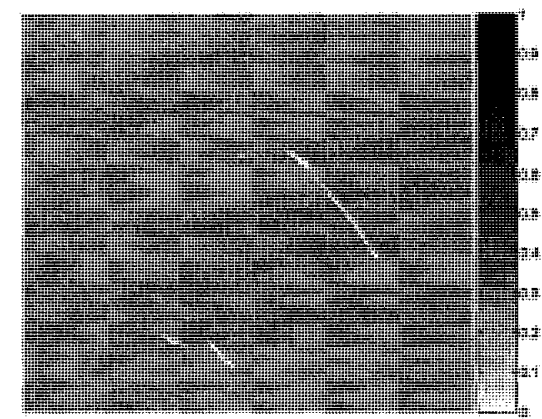

Figure K-10: Experiment \#2 subscan integration quality map

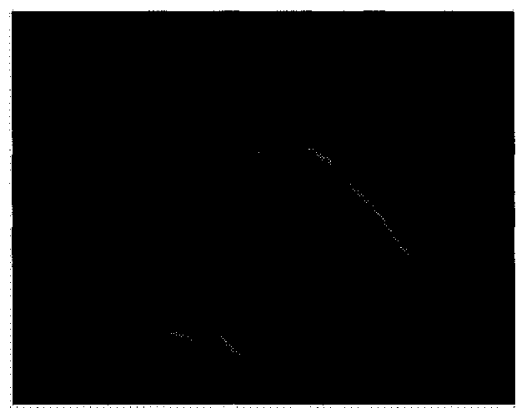

Figure K-12: Experiment \#2 subscan acceptability map 


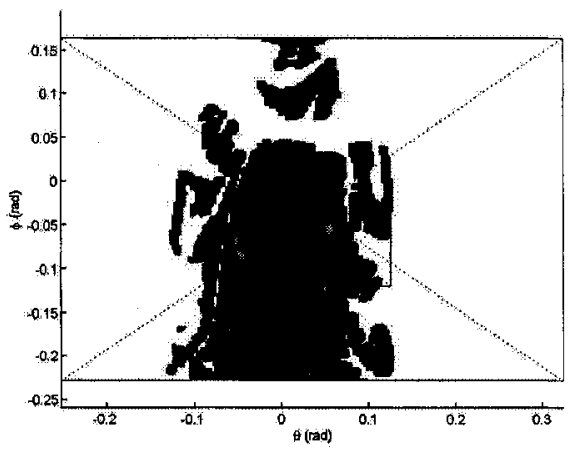

Figure K-13: Experiment \#3 subscan location in anchor

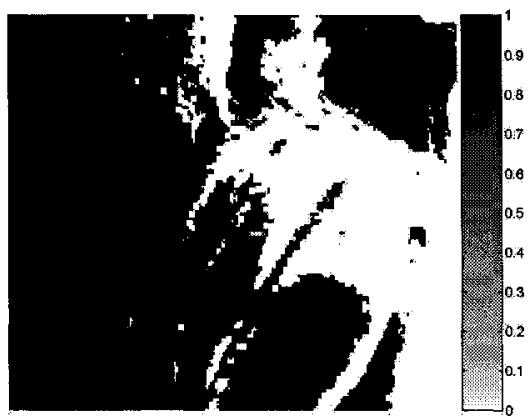

Figure K-15: Experiment \#3 subscan aliasing quality map

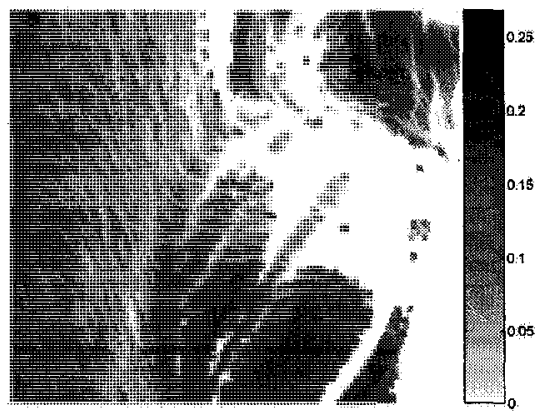

Figure K-17: Experiment \#3 subscan total quality map

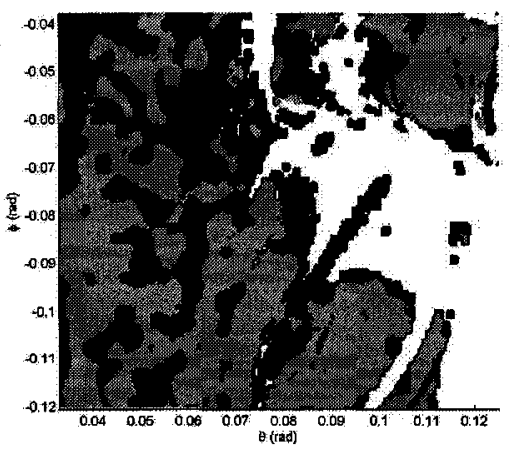

Figure K-14: Experiment \#3 subscan map

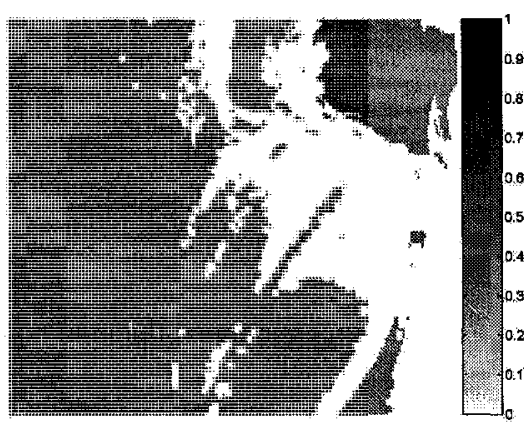

Figure K-16: Experiment \#3 subscan integration quality map

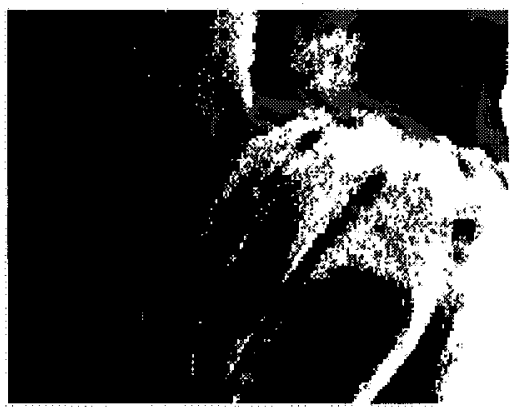

Figure K-18: Experiment \#3 subscan acceptability map 


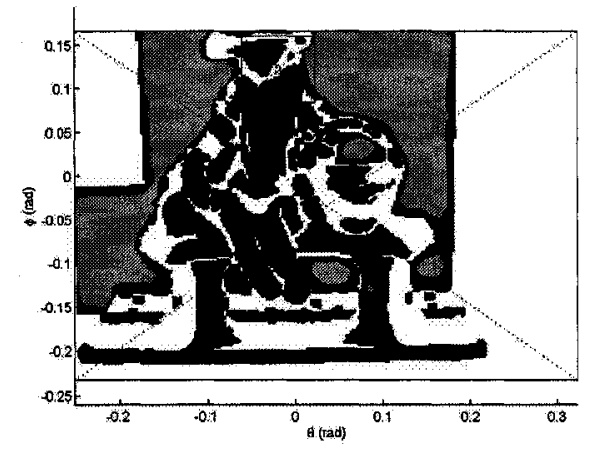

Figure K-19: Experiment \#4 subscan location in anchor

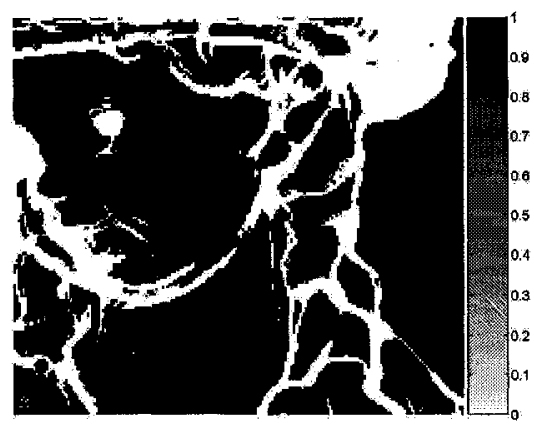

Figure K-21: Experiment \#4 subscan aliasing quality map

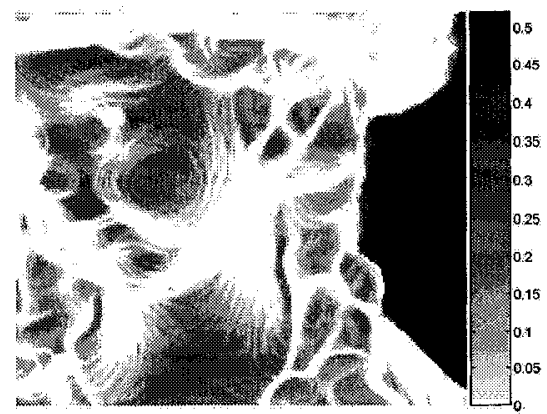

Figure K-23: Experiment \#4 subscan total quality map

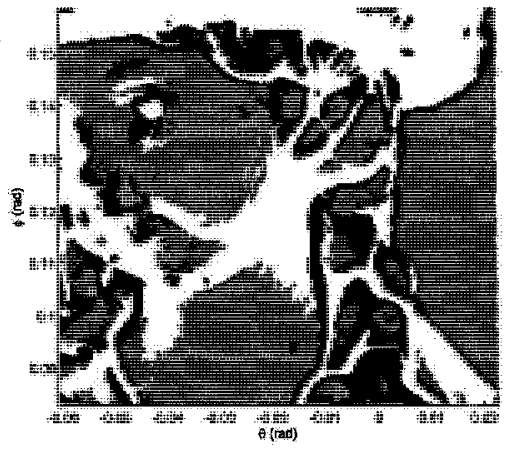

Figure K-20: Experiment \#4 subscan map

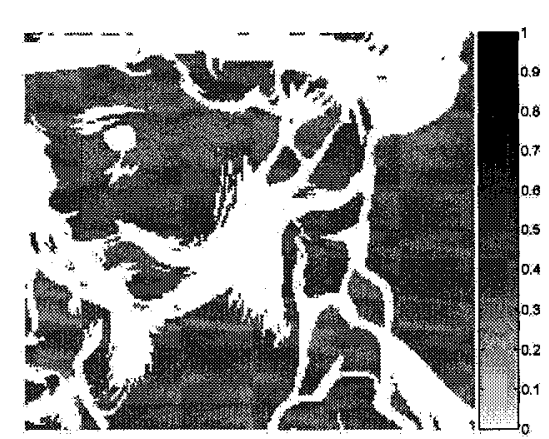

Figure K-22: Experiment \#4 subscan integration quality map

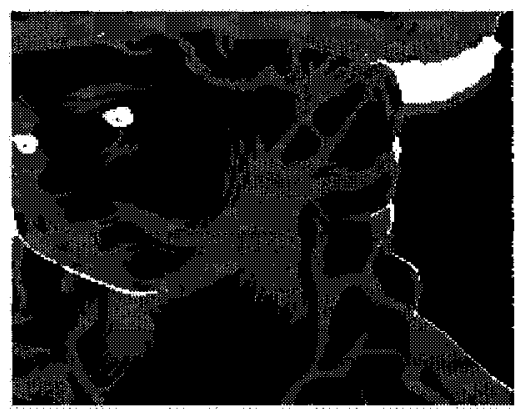

Figure K-24: Experiment \#4 subscan acceptability map 


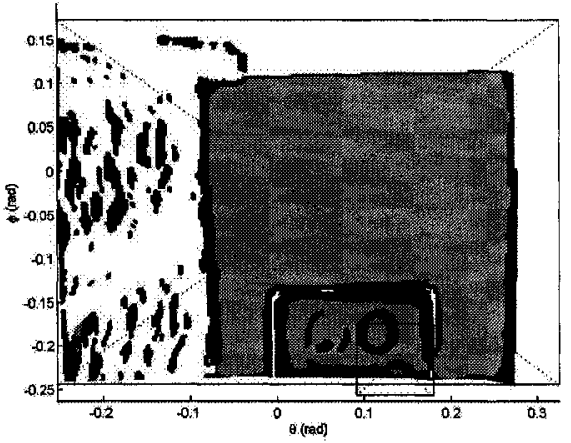

Figure K-25: Experiment \#5 subscan location in anchor

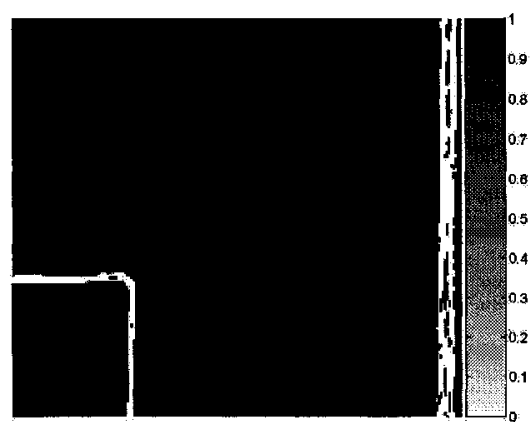

Figure K-27: Experiment \#5 subscan aliasing quality map

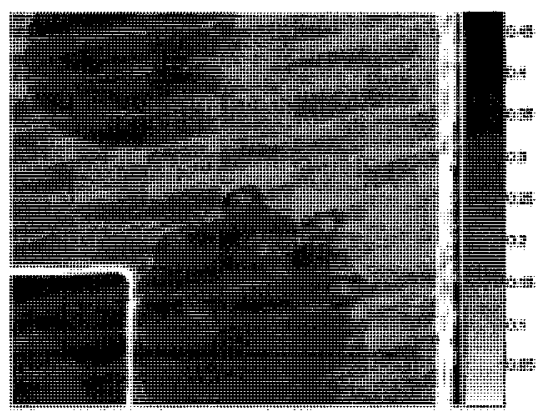

Figure K-29: Experiment \#5 subscan total quality map

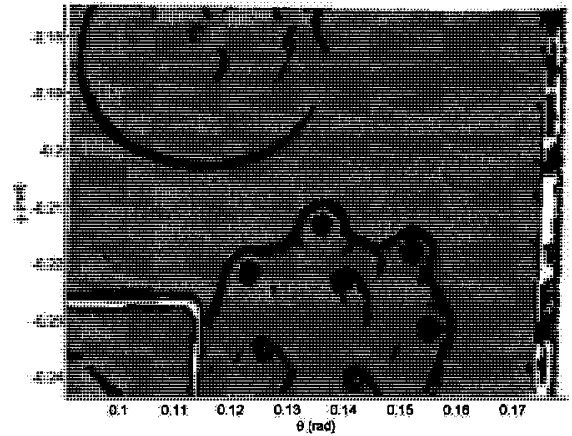

Figure K-26: Experiment \#5 subscan map

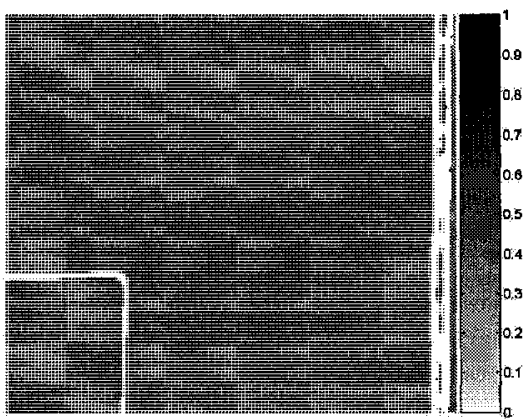

Figure K-28: Experiment \#5 subscan integration quality map

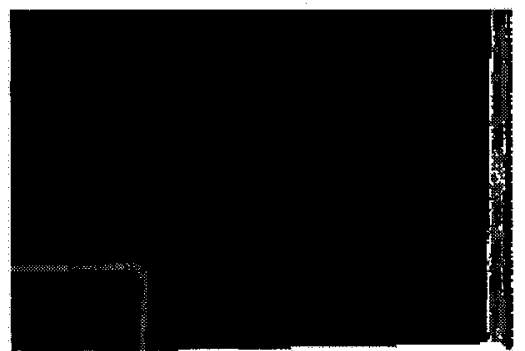

Figure K-30: Experiment \#5 subscan acceptability map 


\section{Appendix L: Subscan Coverage}

Table L-1: Second pass subscan efficiency versus full and reduced scan times and number of measurements (see Table I-1) for target-based scans only with 14-element overlap and $D_{\text {alias }}=0.50$ in Stage 2

\begin{tabular}{|c|c|c|c|c|c|}
\hline Experiment & 1 & 2 & 3 & 4 & 5 \\
\hline \# Anchor Scans & 3 & 1 & 3 & 3 & 3 \\
\hline \# Stage 1 Subscans & 19 & 27 & 16 & 32 & 27 \\
\hline \# Stage 2 Subscans & 41 & 88 & 57 & 89 & 85 \\
\hline \# Measurements & $4,128,768$ & $7,602,176$ & $4,980,736$ & $8,126,464$ & $7,536,640$ \\
\hline Scanning time & $11.09 \mathrm{~min}$ & $20.42 \min$ & $13.02 \mathrm{~min}$ & $21.47 \mathrm{~min}$ & $20.24 \mathrm{~min}$ \\
\hline Processing time & $47.45 \mathrm{~min}$ & $85.93 \mathrm{~min}$ & $54.38 \mathrm{~min}$ & $87.13 \mathrm{~min}$ & $81.56 \mathrm{~min}$ \\
\hline Total Time & $58.54 \mathrm{~min}$ & $106.35 \mathrm{~min}$ & $67.40 \mathrm{~min}$ & $108.60 \mathrm{~min}$ & $101.80 \mathrm{~min}$ \\
\hline \multicolumn{6}{|l|}{ Versus Full Scan } \\
\hline Time Efficiency & $12.8 \%$ & $23.3 \%$ & $14.8 \%$ & $23.8 \%$ & $22.3 \%$ \\
\hline Sampling Efficiency & $16.3 \%$ & $29.9 \%$ & $19.6 \%$ & $32.0 \%$ & $37.4 \%$ \\
\hline \multicolumn{6}{|l|}{ Versus Reduced Scan } \\
\hline Time Efficiency & $30.9 \%$ & $28.6 \%$ & $47.7 \%$ & $38.2 \%$ & $35.8 \%$ \\
\hline Sampling Efficiency & $48.4 \%$ & $45.6 \%$ & $65.4 \%$ & $65.9 \%$ & $47.7 \%$ \\
\hline
\end{tabular}




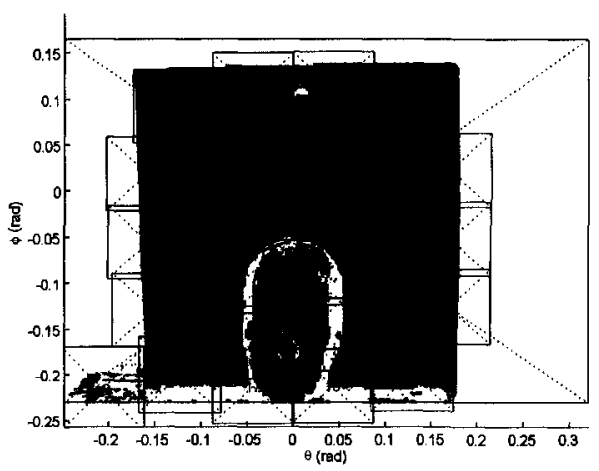

Figure L-1: Experiment \#1 subscan coverage

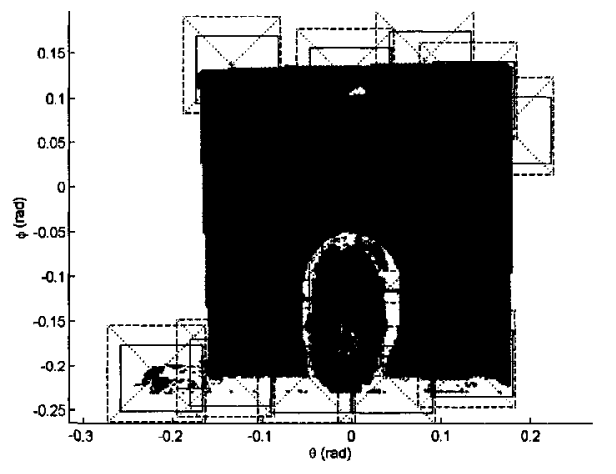

Figure L-3: Experiment \#1 second pass subscans

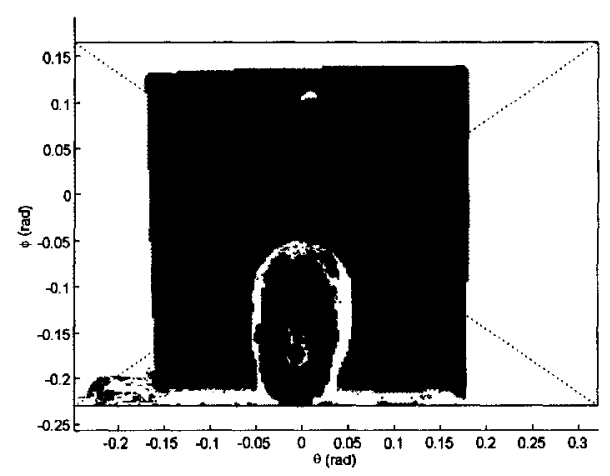

Figure L-2: Experiment \#1 Unscanned region 


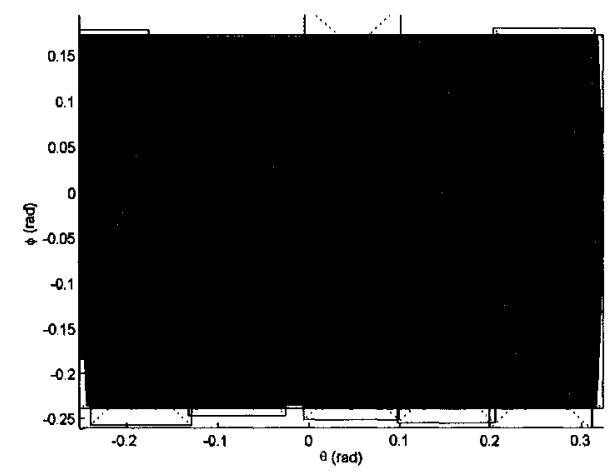

Figure L-4: Experiment \#2 subscan coverage

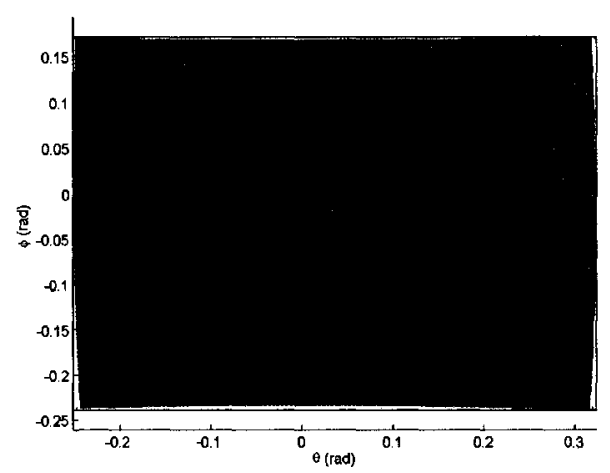

Figure L-5: Experiment \#2 Unscanned region

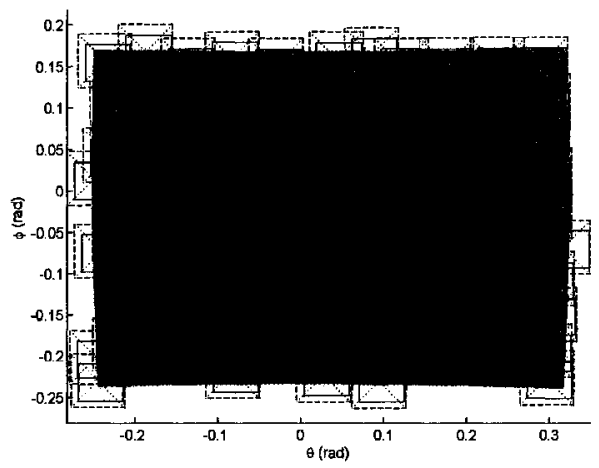

Figure L-6: Experiment \#2 second pass subscans 

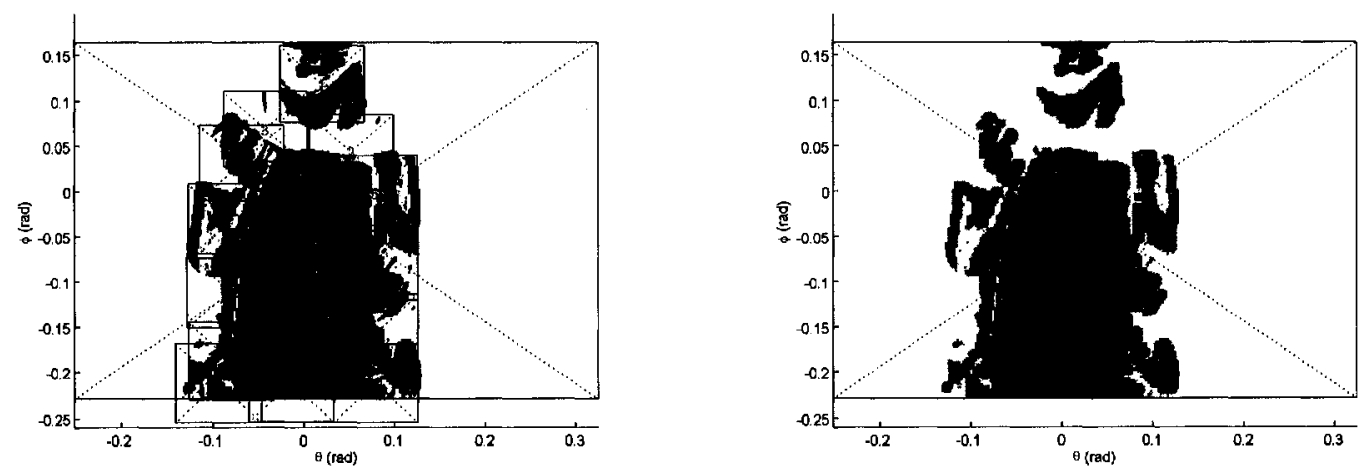

Figure L-7: Experiment \#3 subscan cov-

Figure L-8: Experiment \#3 Unscanned erage region

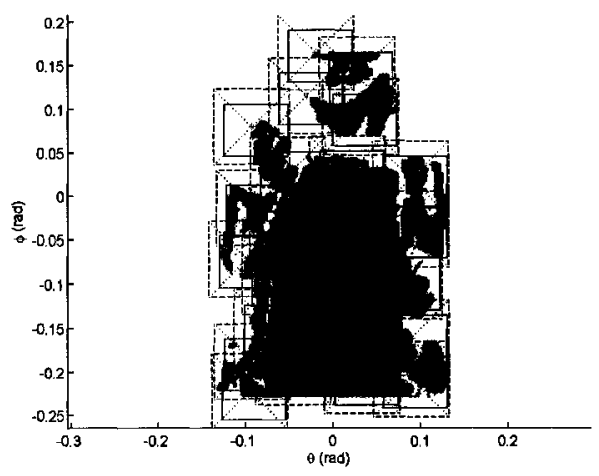

Figure L-9: Experiment \#3 second pass subscans 


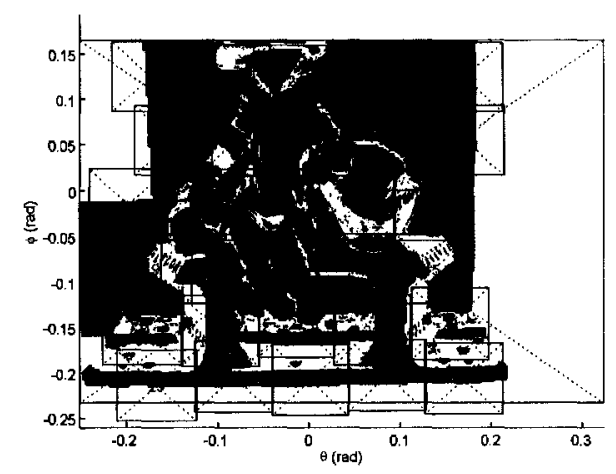

Figure L-10: Experiment \#4 subscan coverage

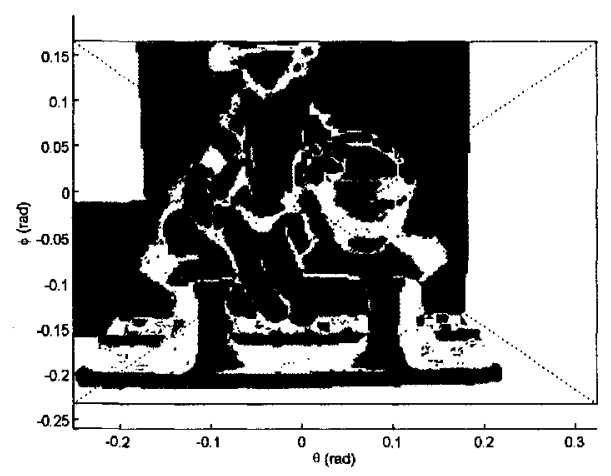

Figure L-11: Experiment \#4 Unscanned region

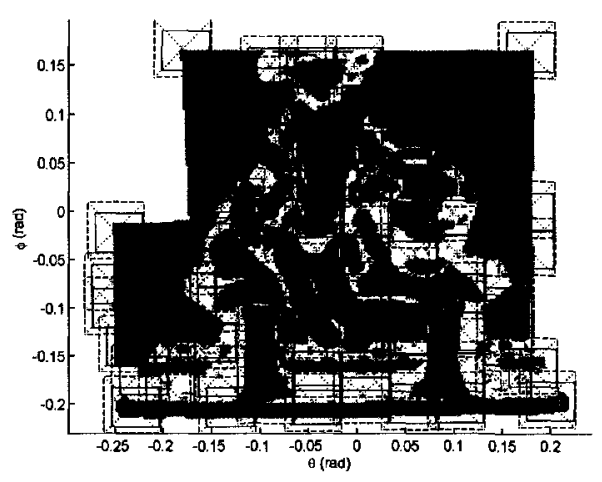

Figure L-12: Experiment \#4 second pass subscans 


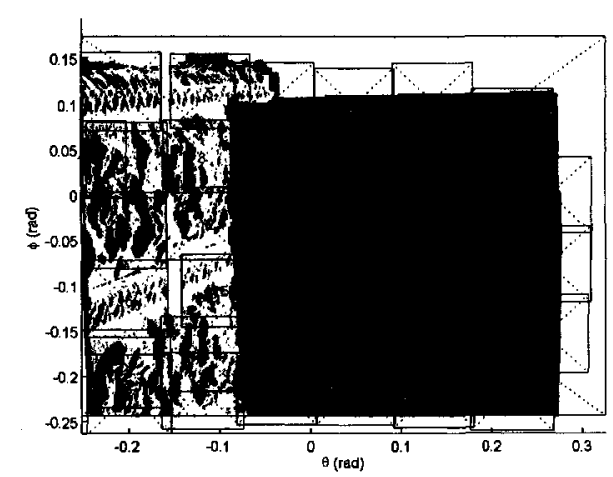

Figure L-13: Experiment \#5 subscan coverage

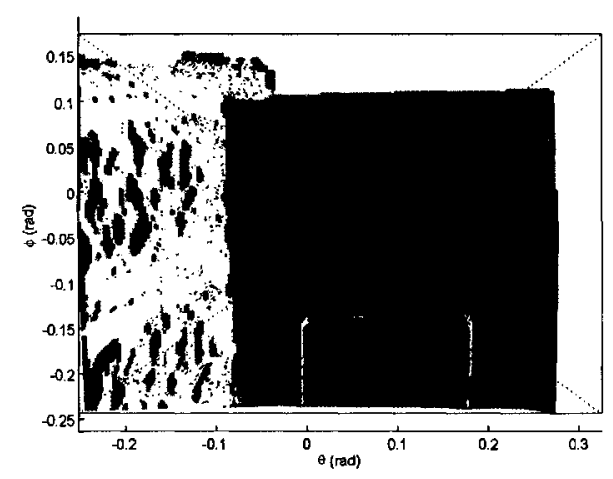

Figure L-14: Experiment \#5 Unscanned region

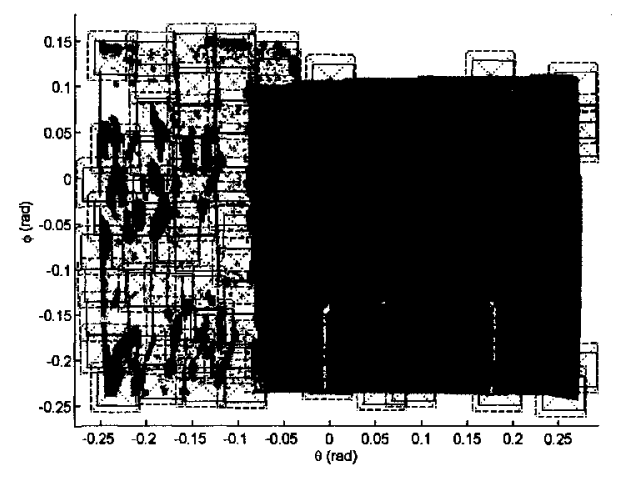

Figure L-15: Experiment \#5 second pass subscans 


\section{Appendix M: Scalar Equivalent}

\section{Variance}

\section{M.1 Distance in Cartesian and Spherical Coordinates}

An important concept when working with spatial measurements is how to determine the distance between them. A point can be represented in either Cartesian or spherical coordinates; that is $\mathbf{p}=[x, y, z]^{T}$ or $\mathbf{p}=[R, \theta, \phi]^{T}$ depending upon the coordinate system used. When $\theta=\phi=0$, then the radial component is directed along the z-axis so that $\mathbf{p}=[0,0, z]^{T}$ or $\mathbf{p}=[R, 0,0]^{T}$. When $\mathbf{p}_{A}$ and $\mathbf{p}_{B}$ are represented in Cartesian coordinates then distance refers to the shortest linear path between $\mathbf{p}_{A}$ and $\mathbf{p}_{B}$. If $\mathbf{p}_{A}$ and $\mathbf{p}_{B}$ are represented in spherical coordinates then distance refers to the shortest geodesic path between $\mathbf{p}_{A}$ and $\mathbf{p}_{B}$ [104]. When the term "distance" is applied to points in Cartesian coordinates, then it refers to the length of the shortest linear path. If the term "distance" is applied to points in spherical coordinates then it refers to the length of the shortest geodesic path. Where coordinate systems are being interchanged, the terms linear distance and geodesic distance are used.

The issues of distance metrics and measurement units can be addressed through an intelligent selection of the measurement space and distance metrics. Consider a bounded region $G \subset \mathbb{R}^{3}$ in which: 
- the rotational components are bounded such that $\theta \leq \min \left\{\theta_{\max }, \pi\right\}$ and $\theta>$ $\max \left\{\theta_{\min },-\pi\right\}$ where $\theta_{\max }$ and $\theta_{\max }$ are the bounds of the total field of view of the scanner along the $\theta$ axis, and $\phi \leq \min \left\{\phi_{\max }, \pi\right\}$ and $\phi>\max \left\{\phi_{\min },-\pi\right\}$ where $\phi_{\max }$ and $\phi_{\max }$ are the bounds of the total field of view of the scanner along the $\phi$ axis. This avoids the problem of two radial measure values representing the same spatial point.

- the radial component is restricted to being larger than zero so that no point is allowed to exist at the origin; that is $R>R_{\min }>0$.

These restrictions define the boundaries of a region $G$ in the Cartesian space $\mathbb{R}^{3}$. Points within this space can be represented in either spherical or Cartesian coordinates.

A norm $\left\{\rho_{G}(\mathbf{x}, \mathbf{y}): \mathbf{x}, \mathbf{y} \in G\right\}$ can be defined on the bounded space $G$ such that

$$
\rho_{G}(\mathbf{x}, \mathbf{y}):=(\mathbf{x}-\mathbf{y})^{T} M^{-1}(\mathbf{x}-\mathbf{y})
$$

where $M$ is a nonsingular $3 \times 3$ weighting matrix. When $M=\Sigma$ where $\Sigma$ is the covariance matrix then $\rho_{G}(\mathbf{x}, \mathbf{y})$ is the Mahalanobis norm or Mahalanobis distance, written here as $d(\mathbf{x} \mid \mathbf{y}, \Sigma)$. This is discussed in greater detail later in this work. If $M=I$ where $I$ is the identity matrix in which the diagonal elements have units of $I_{1,1}$ in metres ${ }^{2}, I_{2,2}$ in radians $^{2}$ and $I_{3,3}$ in radians ${ }^{2}$ then $\rho_{G}(\mathbf{x}, \mathbf{y})$ is the Euclidean norm or Euclidean distance, written here as $\|\mathbf{x}-\mathbf{y}\|_{G}$. Both norms generate unitless distance metrics so are immune to differences in terms among the components of $\mathbf{x}$ and $\mathbf{y}$. As a result, both terms can be expressed in either Cartesian or spherical coordinates. Note that when $\mathbf{x}$ and $\mathbf{y}$ are in spherical coordinates then the Euclidean distance is the geodesic distance, not the linear distance. 


\section{M.2 Mahalanobis Distance}

The Mahalanobis distance is defined by

$$
d^{2}\left(\mathbf{p} \mid \mathbf{p}_{A}, \Sigma_{A}\right)=\left(\mathbf{p}_{A}-\mathbf{p}\right)^{T} \Sigma_{A}^{-1}\left(\mathbf{p}_{A}-\mathbf{p}\right)
$$

where $\Sigma_{A}$ is the covariance matrix associated with surface point $\mathbf{p}_{A}$, and $\sqrt{d^{2}\left(\mathbf{p} \mid \mathbf{p}_{A}, \Sigma_{A}\right)}$ is the distance to some point $\mathbf{p}$ in the environment given a measurement $\mathbf{p}_{A}$ with covariance $\Sigma_{A}$ [193] [194] [195] [146] [196]. The Mahalanobis distance has two useful properties. First, unlike the Euclidean distance, the Mahalanobis distance is unitless, as explained in Section M.1. This property is particularly useful when measuring the distance between points in spherical space because the range and rotational components use different units. The former means that points generated using the Mahalanobis distance metric must have units consistent with the spherical space in which they will be placed. Second, the Mahalanobis distance has a $\chi^{2}$ distribution with a number of degrees of freedom equal to the number of elements in the distance vector [141] [146]. This property can be used to determine whether two measurements are close enough that they are likely to have arisen from nearly the same surface feature so can be merged to generate a new measurement estimate [141].

In (M-2), $\mathbf{p}$ and $\mathbf{p}_{A}$ are measurements represented in spherical coordinates. As a result, the metric represents the distance in spherical space, not Cartesian space. Although the rotation coordinates are closed, the region within which the Mahalanobis distance is calculated is bounded by the maximum viewing angles of the scanner. As a result, there is no risk of $\mathbf{p}$ and $\mathbf{p}_{A}$ being separated by sufficient distance that the rotational components would overlap.

In practice, (M-2) may be further simplified depending upon the relative contributions of each of the axial components. Consider (M-2) for the case in which $\Sigma_{A}$ is represented 
as a diagonal matrix such that

$$
\Sigma_{A}=\left[\begin{array}{ccc}
\sigma_{R_{A}}^{2} & 0 & 0 \\
0 & \sigma_{\theta_{A}}^{2} & 0 \\
0 & 0 & \sigma_{\phi_{A}}^{2}
\end{array}\right]
$$

In this case (M-2) can be reduced to

$$
d^{2}\left(\mathbf{p} \mid \mathbf{p}_{A}, \Sigma_{A}\right)=\frac{\left(R_{A}-R\right)^{2}}{\sigma_{R_{A}}^{2}}+\frac{\left(\theta_{A}-\theta\right)^{2}}{\sigma_{\theta_{A}}^{2}}+\frac{\left(\phi_{A}-\phi\right)^{2}}{\sigma_{\phi_{A}}^{2}}
$$

which is the sum of the Mahalanobis distances along each axis; that is

$$
d^{2}\left(\mathbf{p} \mid \mathbf{p}_{A}, \Sigma_{A}\right)=d^{2}\left(R \mid R_{A}, \sigma_{R_{A}}\right)+d^{2}\left(\theta \mid \theta_{A}, \sigma_{\theta_{A}}\right)+d^{2}\left(\phi \mid \phi_{A}, \sigma_{\phi_{A}}\right)
$$

As a result, the Mahalanobis distance can, in this case, be calculated for each axis separately before being combined, which results in a significant reduction in the number of calculations required to obtain this value. Performing the same calculation in Cartesian coordinates would require significantly more mathematical operations to obtain it.

\section{M.3 Scalar Equivalent Variance}

The calculation of the Mahalanobis distance can be simplified by representing the covariance matrix $\Sigma_{A}$ as a unitless scalar equivalent variance function $\sigma^{2}\left(\mathbf{p} \mid \mathbf{p}_{A}, \Sigma_{A}\right)$ along a unit vector $\mathbf{u}\left(\mathbf{p} \mid \mathbf{p}_{A}\right)$. The scalar equivalent variance is the scalar variance at point $\mathbf{p}$ given a local surface point $\mathbf{p}_{A}$ with covariance $\Sigma_{A}$, and the unit vector is a vector oriented toward $\mathbf{p}$ given a local surface point $\mathbf{p}_{A}$. A graphical representation of the scalar equivalent variance can be seen in Figure M-1. Consider a line from $\mathbf{p}_{A}$ to some point in space $\mathbf{p}$ as shown in Figure M-1. If the variance of a cross-section of $\Sigma_{A}$ along the shortest path in spherical space from $\mathbf{p}_{A}$ to $\mathbf{p}$ can be represented by a scalar variance $\sigma^{2}\left(\mathbf{p} \mid \mathbf{p}_{A}, \Sigma_{A}\right)$ then (M-2) can be rewritten as

$$
d^{2}\left(\mathbf{p} \mid \mathbf{p}_{A}, \sigma^{2}\left(\mathbf{p} \mid \mathbf{p}_{A}, \Sigma_{A}\right)\right)=\frac{\left\|\mathbf{p}_{A}-\mathbf{p}\right\|_{G}^{2}}{\sigma^{2}\left(\mathbf{p} \mid \mathbf{p}_{A}, \Sigma_{A}\right)}
$$


where $\sigma^{2}\left(\mathbf{p} \mid \mathbf{p}_{A}, \Sigma_{A}\right)$ is the variance of the Gaussian distribution around $\mathbf{p}_{A}$ along a line segment in spherical space from $\mathbf{p}_{A}$ to $\mathbf{p} .\left\|\mathbf{p}_{A}-\mathbf{p}\right\|_{G}^{2}$ is the Euclidean distance between $\mathbf{p}_{A}$ and $\mathbf{p}$ where $\mathbf{p}_{A}, \mathbf{p} \in G$ and $G$ is a bounded region defined in Section M.1.

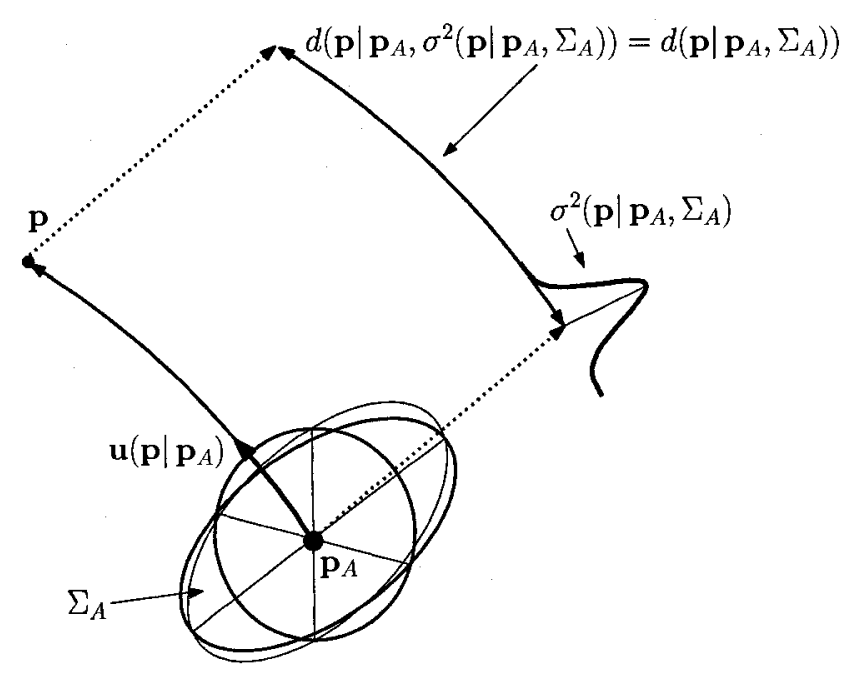

Figure M-1: The scalar equivalent variance (SEV) $\sigma^{2}\left(\mathbf{p} \mid \mathbf{p}_{A}, \Sigma_{A}\right)$ is the variance along shortest line segment in spherical space to $\mathbf{p}$ given starting point $\mathbf{p}_{A}$ with covariance $\Sigma_{A}$, and $d\left(\mathbf{p} \mid \mathbf{p}, \sigma^{2}\left(\mathbf{p} \mid \mathbf{p}_{A}, \Sigma_{A}\right)\right)$ is the Mahalanobis distance to $\mathbf{p}$ given starting point $\mathbf{p}_{A}$ and scalar equivalent variance $\sigma^{2}\left(\mathbf{p} \mid \mathbf{p}_{A}, \Sigma_{A}\right)$.

The scalar variance equivalent form is relatively easy to conceptualize when all the elements of the local surface points use the same units of measure; however, in the spherical coordinate system the rotational and radial components have different units of measure. The Mahalanobis distance is a unitless quantity so the elements of the points used to calculate the Mahalanobis distance can be treated as unitless, and the scalar equivalent variance becomes a weighting factor for the unitless Euclidean distance between two points. 
Both (M-2) and (M-6) express the same distance metric so they can be combined such that

$$
\frac{\left\|\mathbf{p}_{A}-\mathbf{p}\right\|_{G}^{2}}{\sigma^{2}\left(\mathbf{p} \mid \mathbf{p}_{A}, \Sigma_{A}\right)}=\left(\mathbf{p}_{A}-\mathbf{p}\right)^{T} \Sigma_{A}^{-1}\left(\mathbf{p}_{A}-\mathbf{p}\right)
$$

Rearranging the terms results in

$$
\frac{1}{\sigma^{2}\left(\mathbf{p} \mid \mathbf{p}_{A}, \Sigma_{A}\right)}=\frac{\left(\mathbf{p}_{A}-\mathbf{p}\right)^{T}}{\left\|\mathbf{p}_{A}-\mathbf{p}\right\|_{G}} \Sigma_{A}^{-1} \frac{\left(\mathbf{p}_{A}-\mathbf{p}\right)}{\left\|\mathbf{p}_{A}-\mathbf{p}\right\|_{G}}
$$

in which the first and last terms are unit vectors. These terms can be replaced with a single unit vector $\mathbf{u}\left(\mathbf{p} \mid \mathbf{p}_{A}\right)$. (M-8) can be rewritten as

$$
\sigma^{2}\left(\mathbf{p} \mid \mathbf{p}_{A}, \Sigma_{A}\right)=\frac{1}{\mathbf{u}^{T}\left(\mathbf{p} \mid \mathbf{p}_{A}\right) \Sigma_{A}^{-1} \mathbf{u}\left(\mathbf{p} \mid \mathbf{p}_{A}\right)}
$$

where

$$
\mathbf{u}\left(\mathbf{p} \mid \mathbf{p}_{A}\right)=\frac{\mathbf{p}_{A}-\mathbf{p}}{\left\|\mathbf{p}_{A}-\mathbf{p}\right\|_{G}}
$$

Intuitively, (M-9) represents the variance in the direction indicated by the unit vector when the unit vector is applied to the covariance matrix. Now, given a known scalar equivalent variance, the Mahalanobis distance can be calculated using (M-6). 


\section{Appendix N: Subscan Merger}




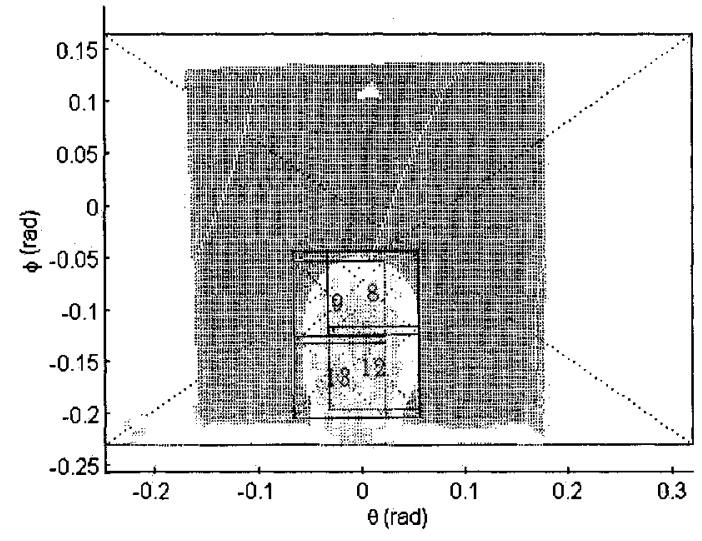

Figure N-1: Experiment \#1 subscans used to illustrate measurement merger

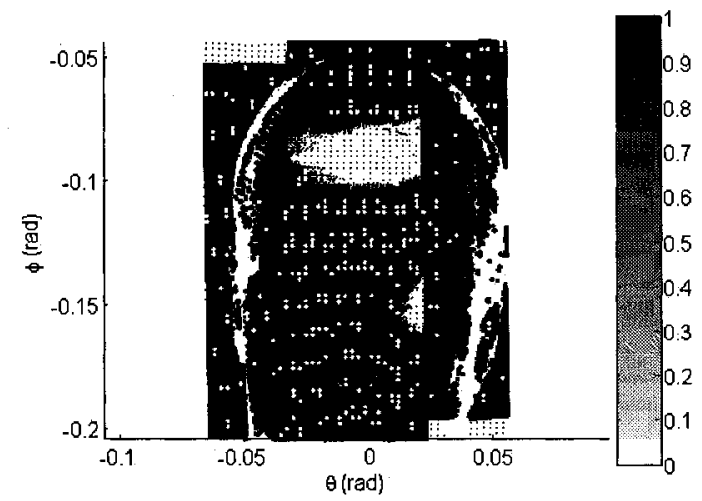

Figure N-3: Experiment \#1 repeatability quality map

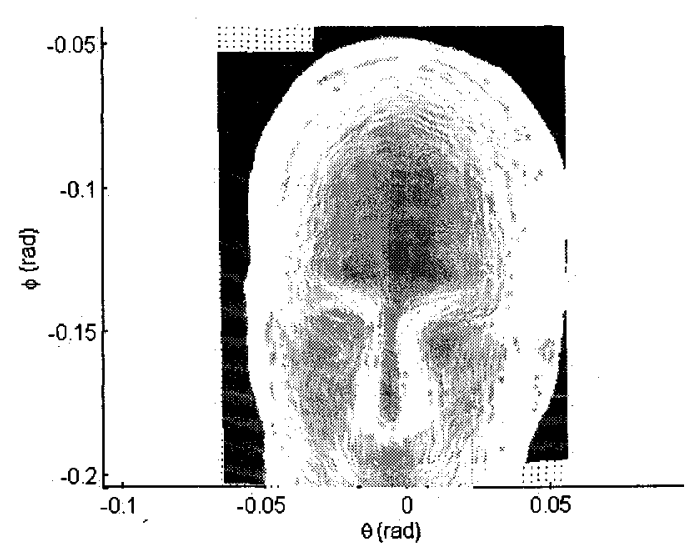

Figure N-2: Close-up of subscan measurements for Experiment \#1 superimposed over anchor scan measurements

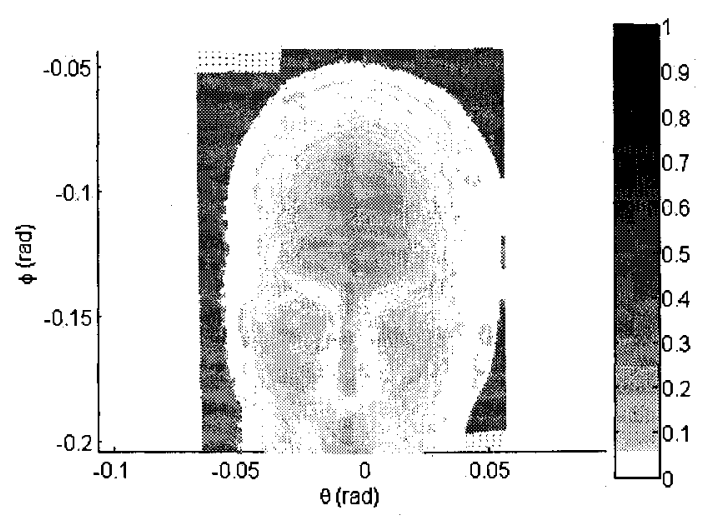

Figure N-4: Experiment \#1 within-scan total quality map 


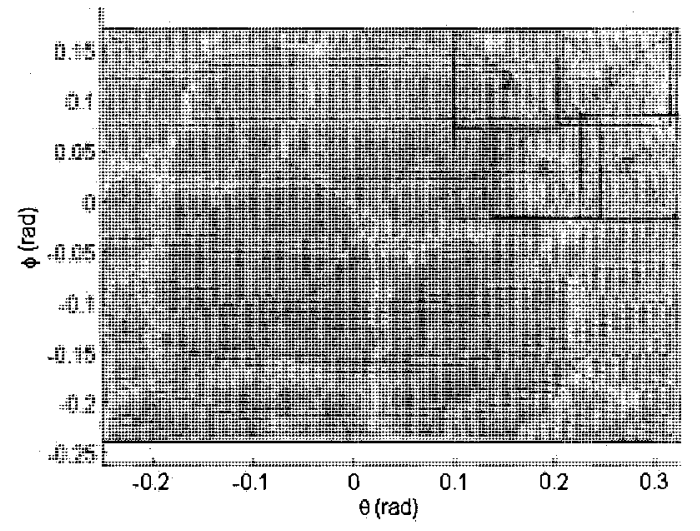

Figure N-5: Experiment \#2 subscans used to illustrate measurement merger

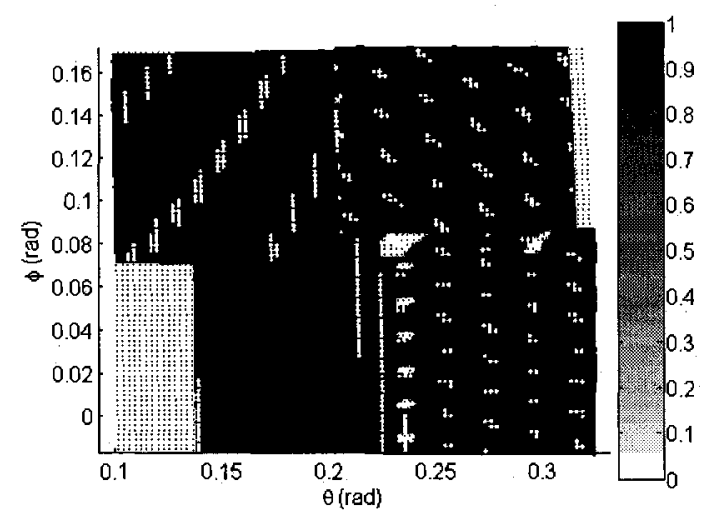

Figure N-7: Experiment \#2 repeatability quality map

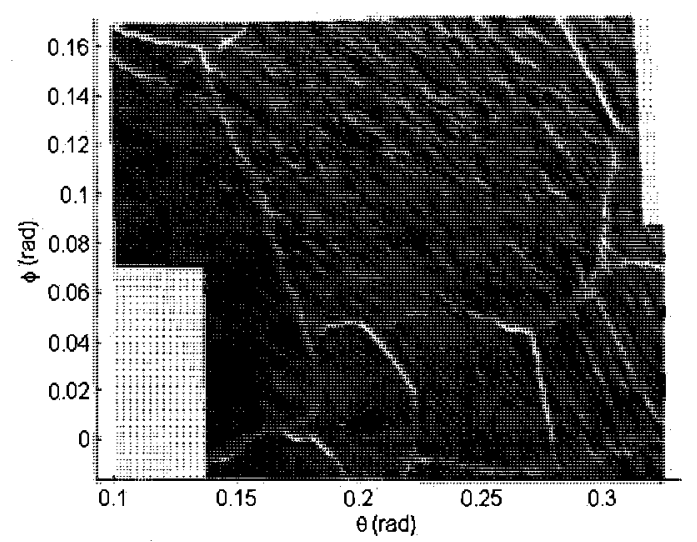

Figure N-6: Close-up of subscan measurements for Experiment \#2 superimposed over anchor scan measurements

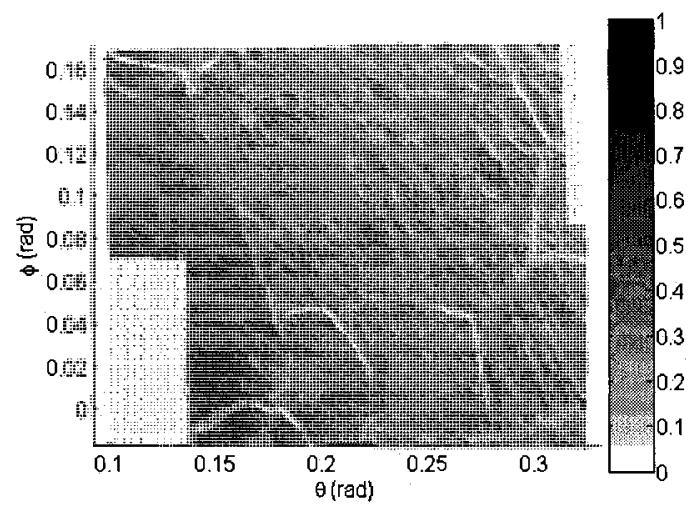

Figure N-8: Experiment \#2 within-scan total quality map 


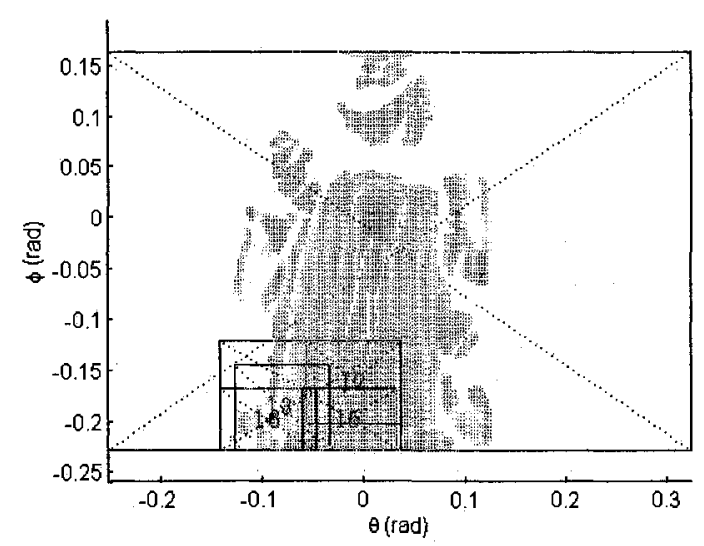

Figure N-9: Experiment \#3 subscans used to illustrate measurement merger

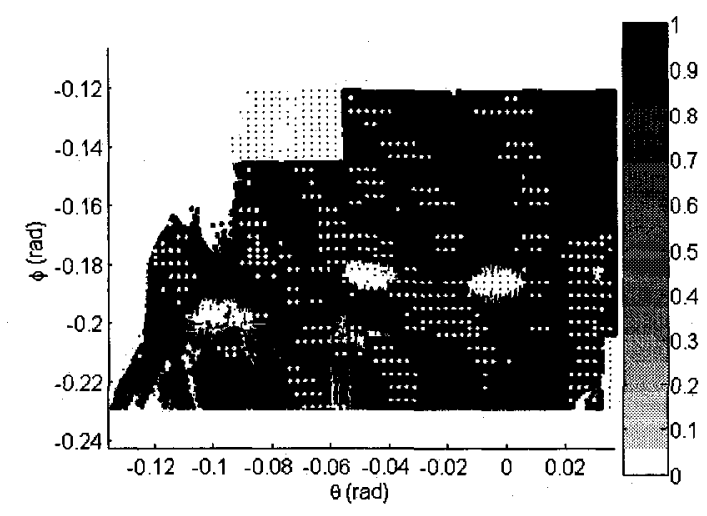

Figure N-11: Experiment \#3 repeatability quality map

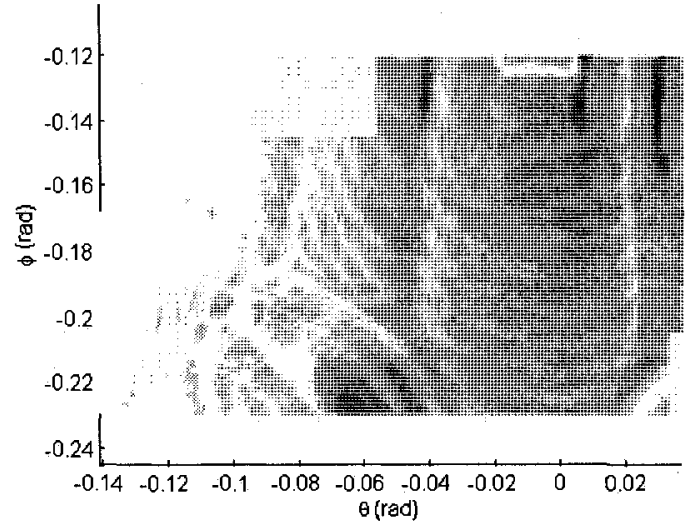

Figure N-10: Close-up of subscan measurements for Experiment \#3 superimposed over anchor scan measurements

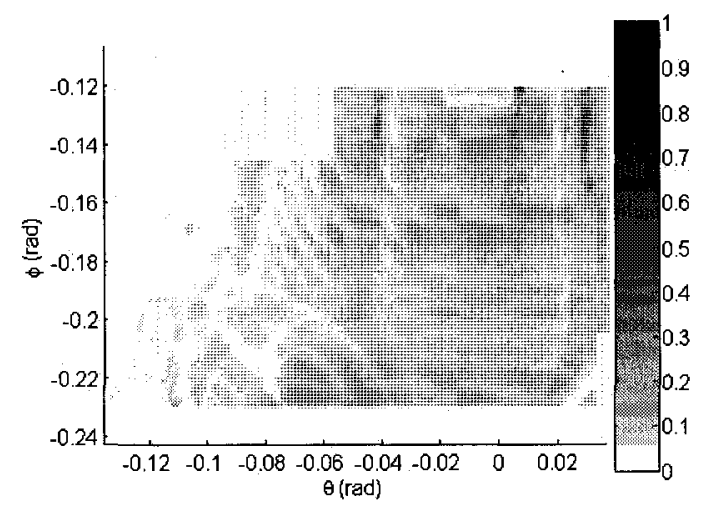

Figure N-12: Experiment \#3 within-scan total quality map 


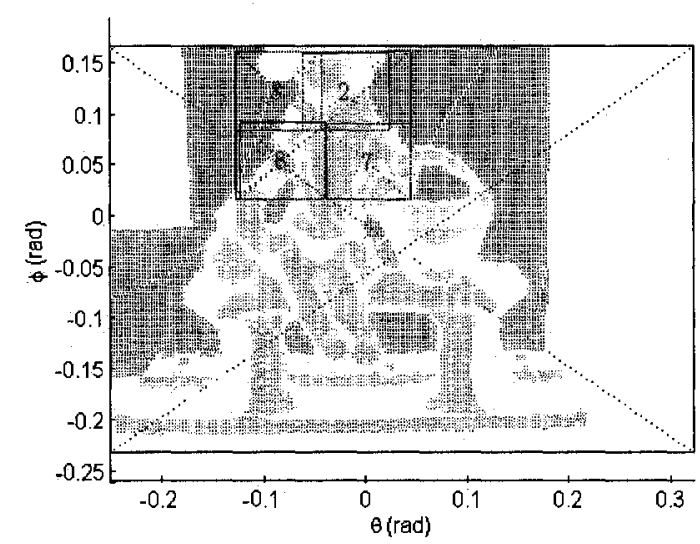

Figure N-13: Experiment \#4 subscans used to illustrate measurement merger

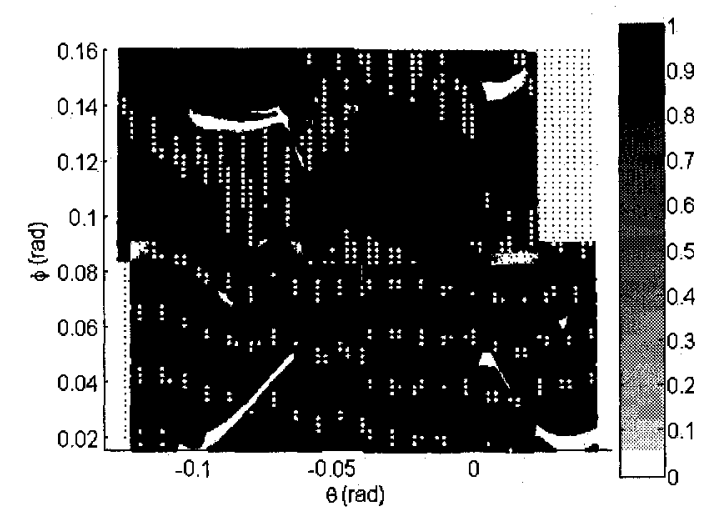

Figure N-15: Experiment \#4 repeatability quality map

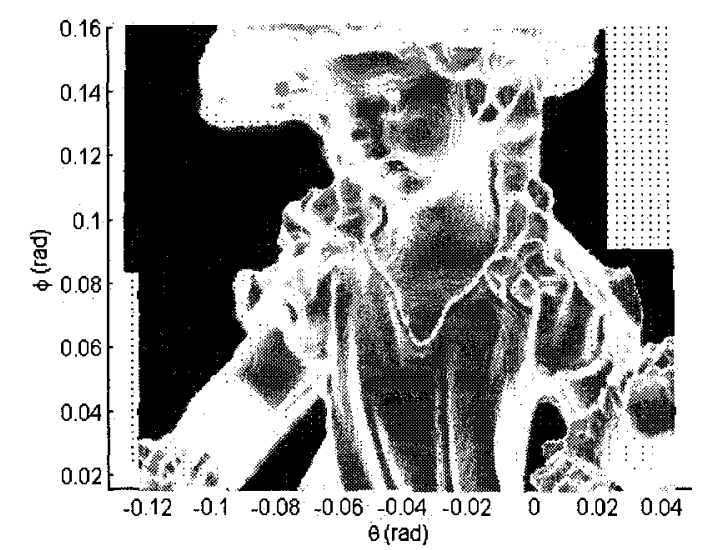

Figure N-14: Close-up of subscan measurements for Experiment \#4 superimposed over anchor scan measurements

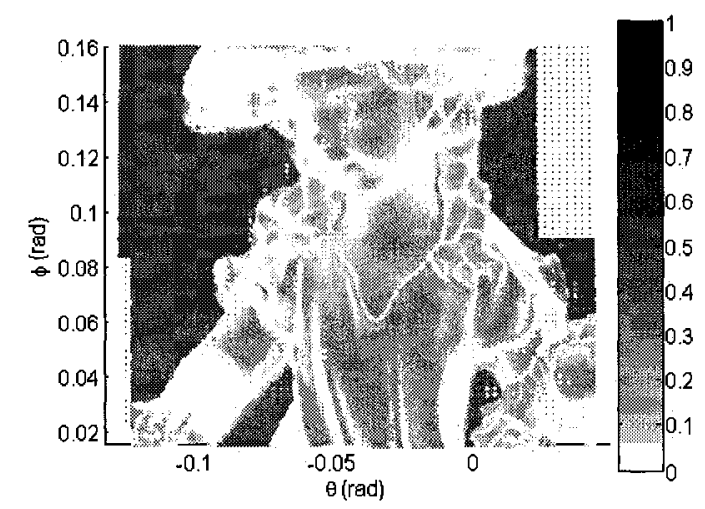

Figure N-16: Experiment \#4 within-scan total quality map 


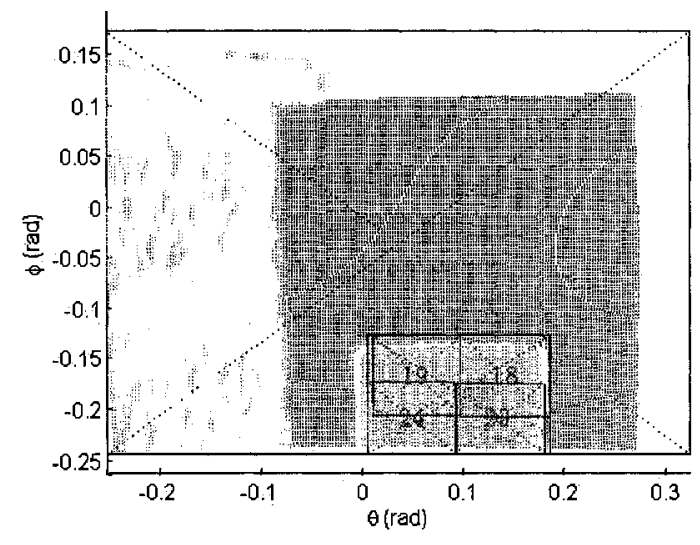

Figure N-17: Experiment \#5 subscans used to illustrate measurement merger

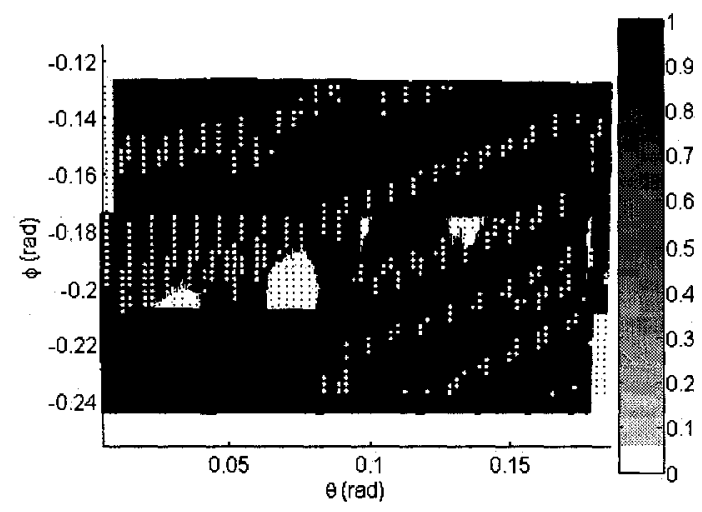

Figure N-19: Experiment \#5 repeatability quality map

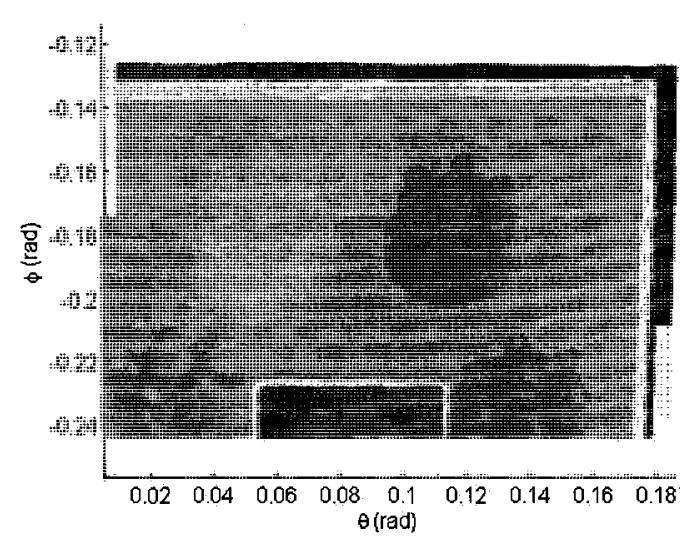

Figure N-18: Close-up of subscan measurements for Experiment \#5 superimposed over anchor scan measurements

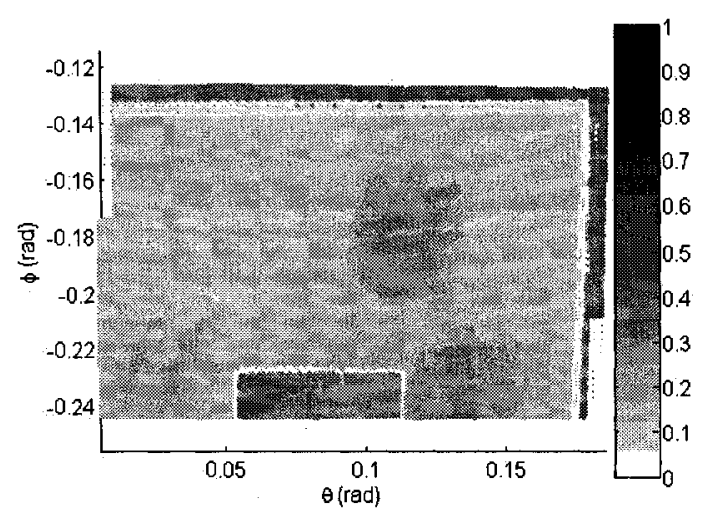

Figure N-20: Experiment \#5 within-scan total quality map 


\section{Appendix O: Within-composite Quality Metrics}




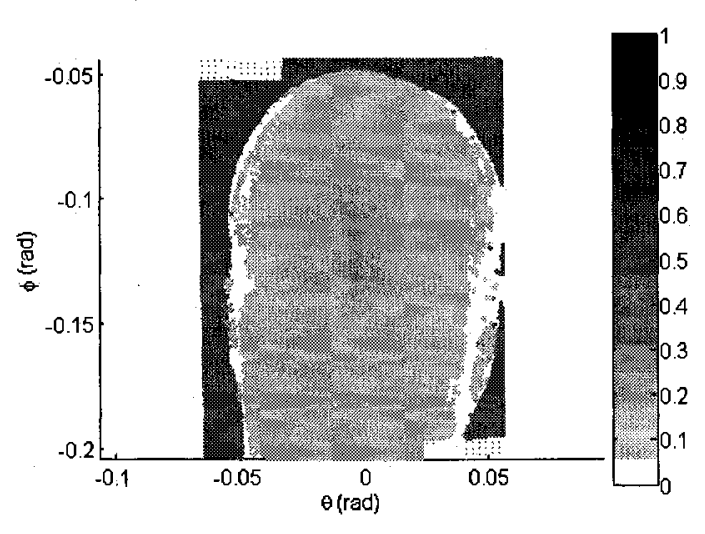

Figure 0-1: Experiment \#1 reflectivity quality map

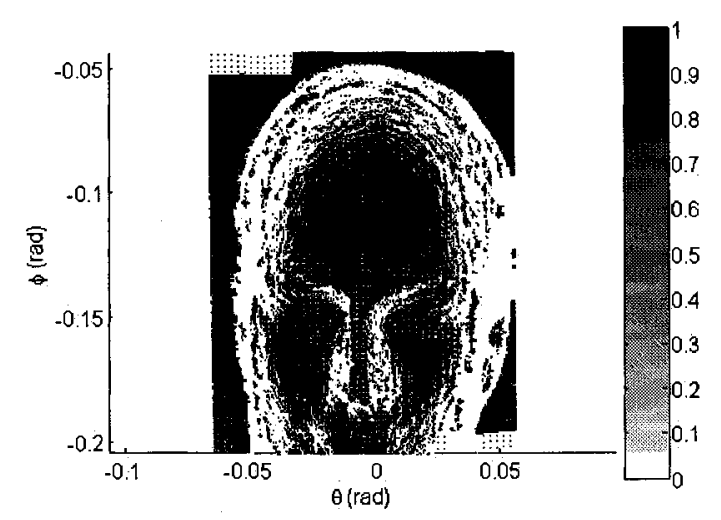

Figure O-3: Experiment \#1 aliasing quality map

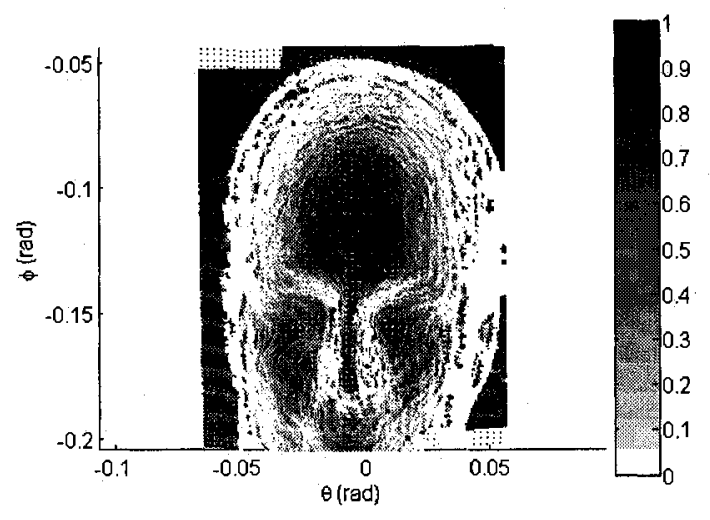

Figure O-2: Experiment \#1 orientation quality map

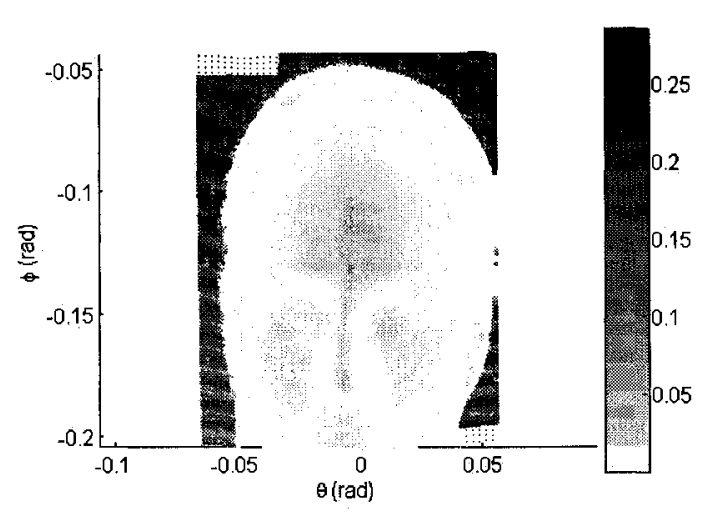

Figure O-4: Experiment \#1 withincomposite total quality map 


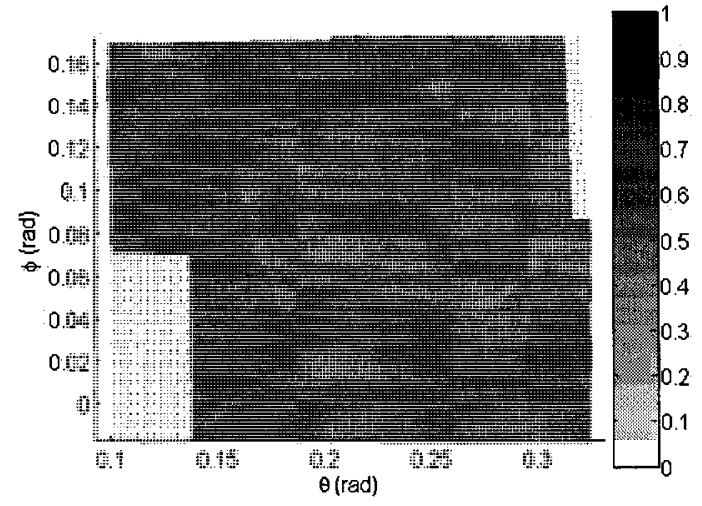

Figure 0-5: Experiment \#2 reflectivity quality map

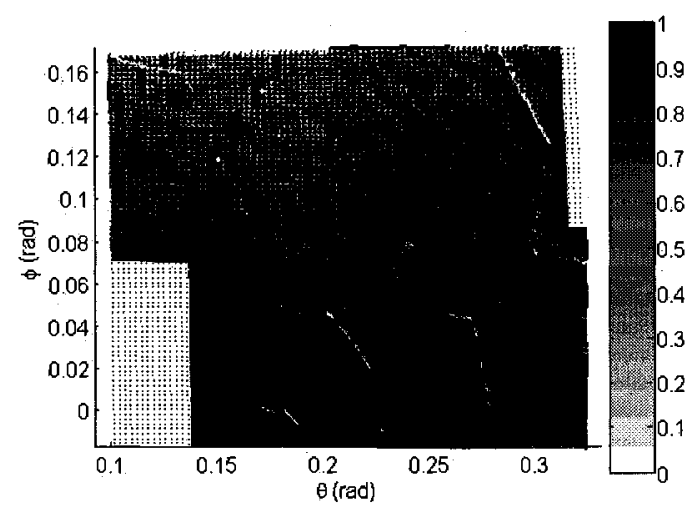

Figure O-7: Experiment \#2 aliasing quality map

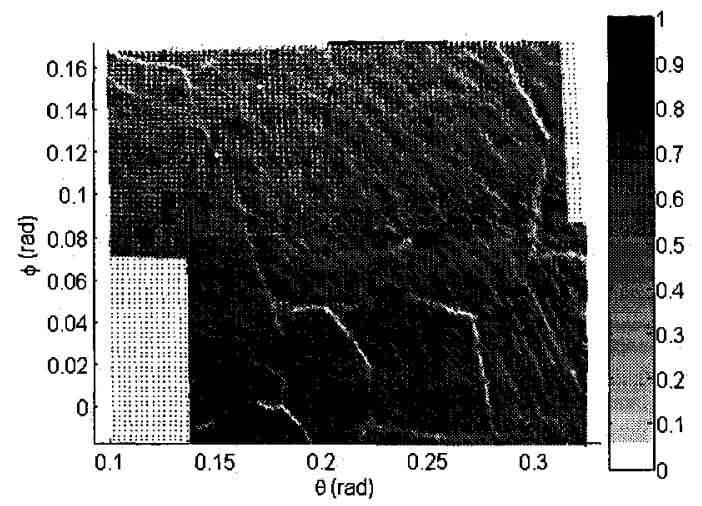

Figure 0-6: Experiment \#2 orientation quality map

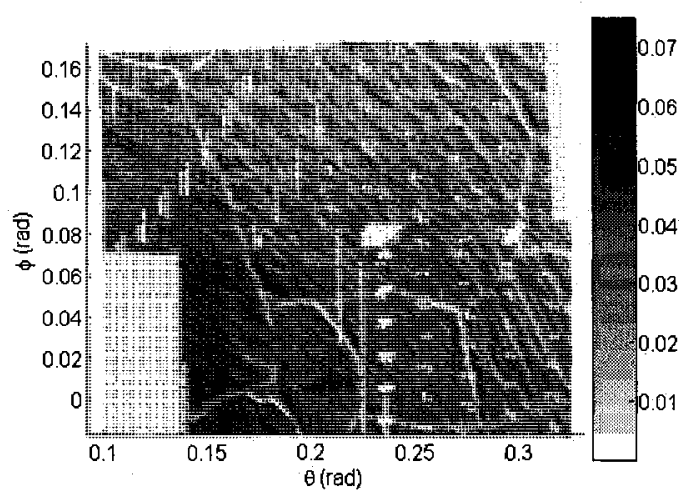

Figure O-8: Experiment \#2 withincomposite total quality map 


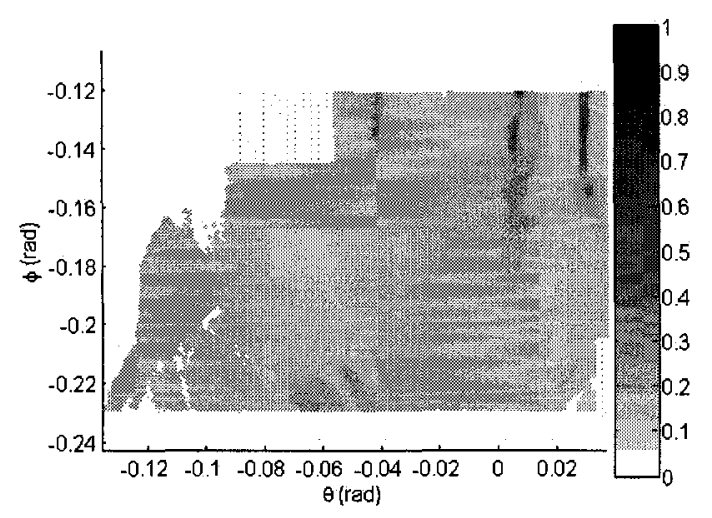

Figure O-9: Experiment \#3 reflectivity quality map

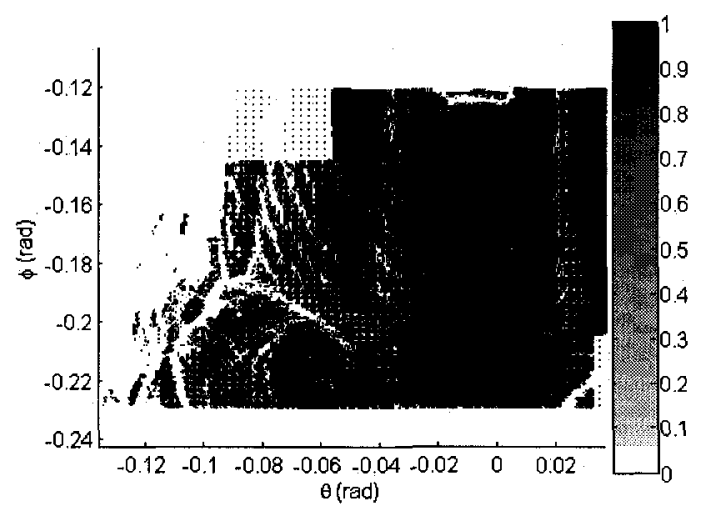

Figure O-11: Experiment \#3 aliasing quality map

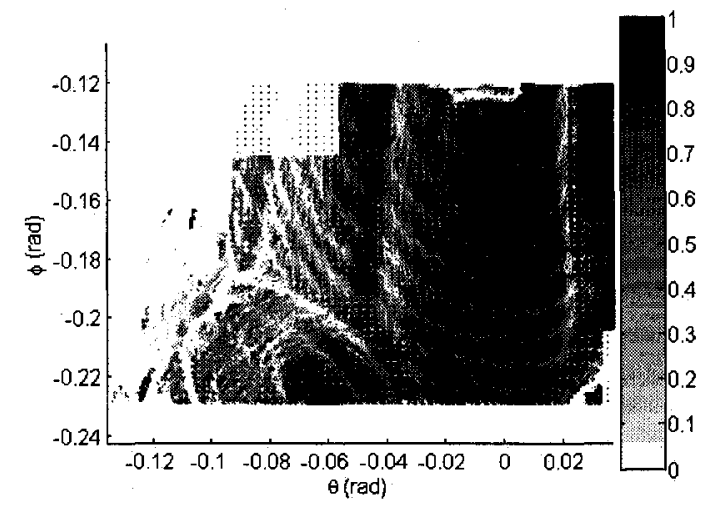

Figure O-10: Experiment \#3 orientation quality map

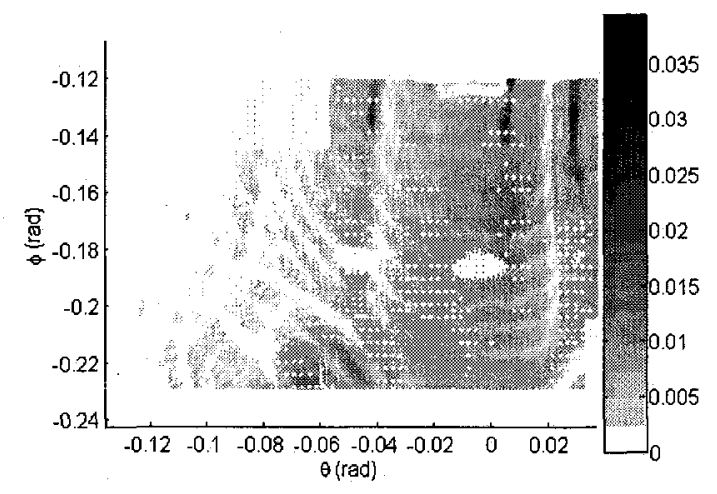

Figure O-12: Experiment \#3 withincomposite total quality map 


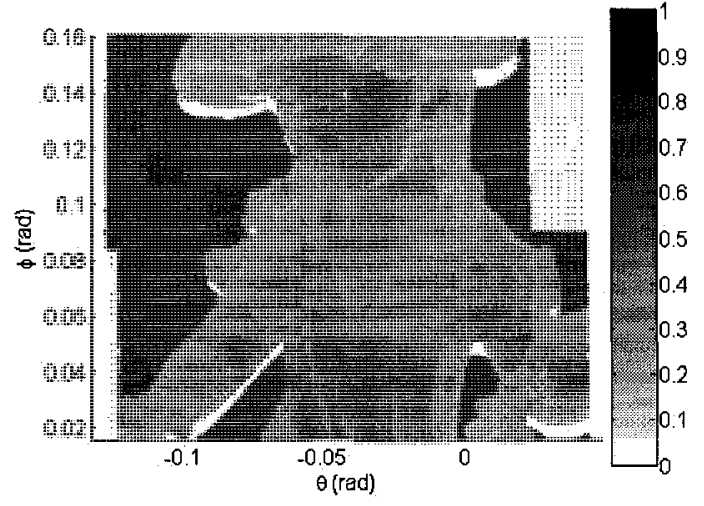

Figure O-13: Experiment \#4 reflectivity quality map

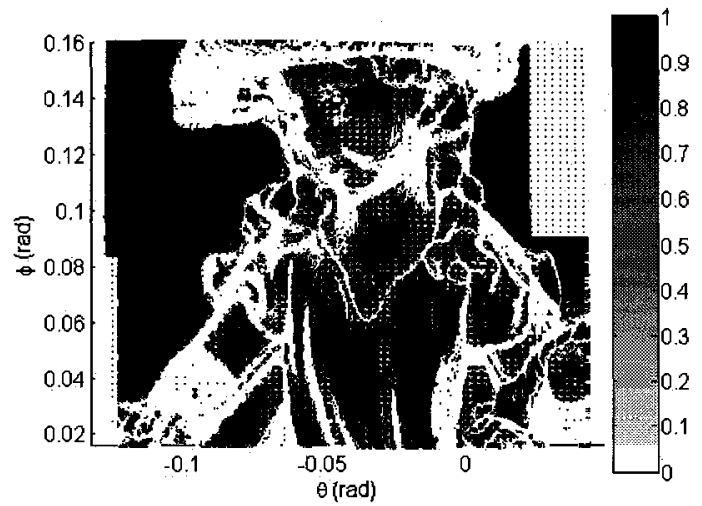

Figure O-15: Experiment \#4 aliasing quality map

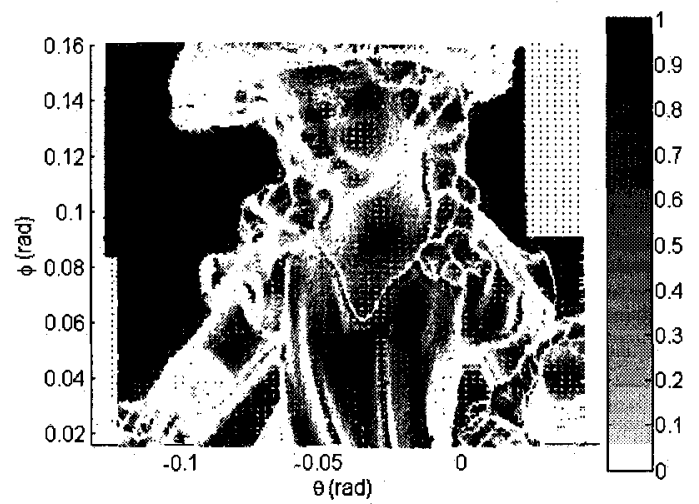

Figure O-14: Experiment \#4 orientation quality map

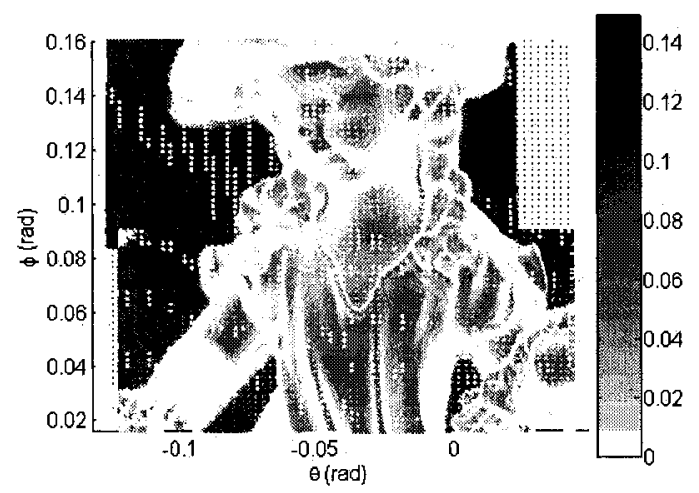

Figure O-16: Experiment \#4 withincomposite total quality map 


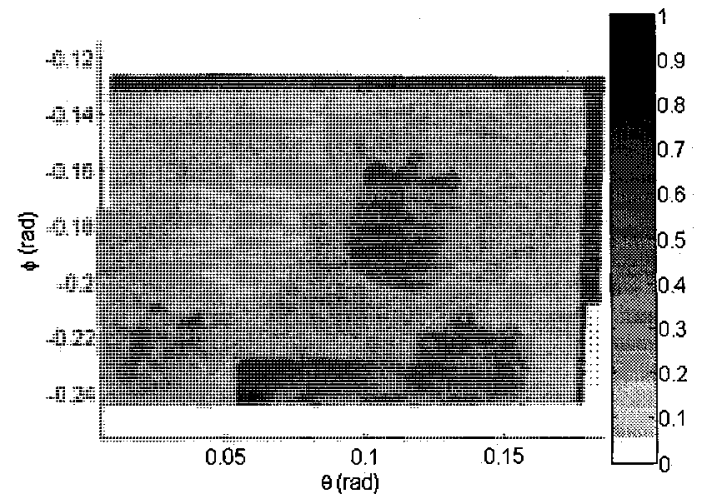

Figure O-17: Experiment \#5 reflectivity quality map

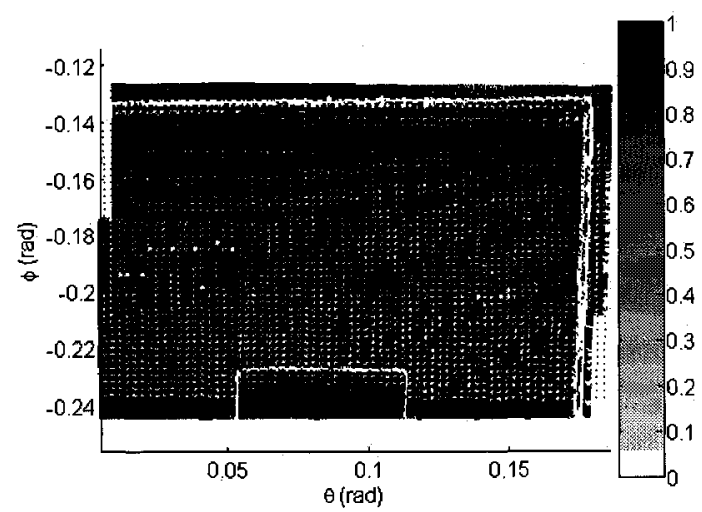

Figure O-19: Experiment \#5 aliasing quality map

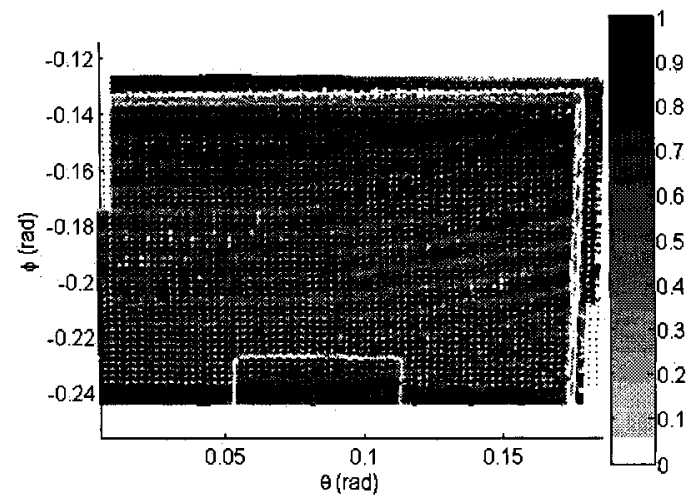

Figure O-18: Experiment \#5 orientation quality map

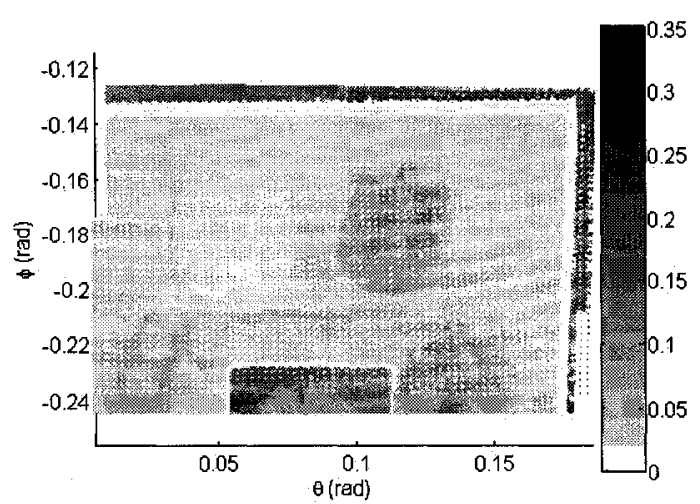

Figure O-20: Experiment \#5 withincomposite total quality map 